\title{
BIRATIONAL CALABI-YAU MANIFOLDS HAVE THE SAME SMALL QUANTUM PRODUCTS
}

\author{
MARK MCLEAN
}

\begin{abstract}
We show that any two birational projective Calabi-Yau manifolds have isomorphic small quantum cohomology algebras after a certain change of Novikov rings. The key tool used is a version of an algebra called symplectic cohomology, which is constructed using Hamiltonian Floer cohomology. Morally, the idea of the proof is to show that both small quantum products are identical deformations of symplectic cohomology of some common open affine subspace.
\end{abstract}

\section{CONTENTS}

1. Introduction 2

1.1. Example 4

1.2. Sketch of Proof 4

1.3. Notation Throughout this paper 8

2. Hamiltonian Floer Cohomology and Filtrations 8

2.1. Alternative Action Values of Periodic Orbits. 8

2.2. Floer Trajectories. 13

2.3. Directed and Inverse Systems 18

2.4. Novikov Rings. 24

2.5. Definition of Floer Cohomology using Alternative Filtrations. 25

2.6. Continuation Maps 28

2.7. Action Maps. 33

2.8. Invariance Under Time Reparameterization. 34

2.9. The pair of pants product 35

3. Floer Cohomology for Lower Semi-Continuous Hamiltonians 36

3.1. Main Definitions. $\quad 36$

3.2. Continuation Maps 38

3.3. Action Maps. 39

3.4. Invariance Under Time Reparameterization. 40

3.5. Pair of Pants Product 41

4. Definition of Symplectic Cohomology 41

5. Properties of Symplectic Cohomology 44

5.1. Changing Contact Cylinders 44

5.2. Partial Independence of the Hamiltonian. 45

5.3. Relation with Quantum Cup Product 47

5.4. Stably Displaceable Complements 48

5.5. Symplectic Cohomology and Alternative Filtrations.

5.6. Transfer Isomorphisms between Index bounded Liouville Domains. 60

5.7. A Chain Complex For Symplectic Cohomology 63

5.8. Changing Novikov Rings. 66 
6. Symplectic Geometry of Projective Varieties and Singular Ample Divisors. 67

6.1. Constructing Appropriate Kähler Forms. 67

6.2. Constructing Index Bounded Contact Cylinders. 71

6.3. Divisors are Stably Displaceable 80

7. Proof of the Main Theorem 88

8. Appendix A: Hamiltonians and Almost Complex Structures Compatible with $\begin{array}{ll}\text { Contact Cylinders. } & 93\end{array}$

9. Appendix B: Avoiding Codimension $\geq 4$ Submanifolds. $\quad 95$

10. Appendix C: Floer trajectories, Filtrations and Compactness. 102

11. Appendix D: Flatness of Novikov rings. 105

$\begin{array}{ll}\text { References } & 109\end{array}$

\section{INTRODUCTION}

We are interested in the following broad question.

Question. What properties do birationally equivalent Calabi-Yau manifolds have in common?

Recall that two smooth projective varieties $X, \widehat{X}$ are birationally equivalent if there exist Zariski dense open subsets $A \subset X, \widehat{A} \subset \widehat{X}$ and an isomorphism $A \stackrel{\cong}{\longrightarrow} \widehat{A}$. In this paper, by Calabi-Yau manifold, we will mean a smooth projective variety with trivial first Chern class. Batyrev showed in [Bat99] that birational Calabi-Yau manifolds have equal Betti numbers. More generally, by combining ideas from [Kon95],[DL01] with ideas in [GS96, Section 3.3], it can be shown that they have identical integral cohomology groups. One can ask, do their cup product structures agree? It turns out that this is false ([Fri91, Example 7.7]). However, Morrison in [Mor96] conjectured that birational Calabi-Yau threefolds have identical small quantum cohomology rings, and this was proven in [LR01]. This leads to the following conjecture.

Conjecture 1.1. ([Rua99]) Any two birational Calabi-Yau manifolds have isomorphic big quantum cohomology rings up to analytic continuation.

It was shown in [LLW16a], [LLW16b] and [LLQW16] that certain birational isomorphisms called ordinary flops induce isomorphisms between big quantum cohomology rings up to analytic continuation. In [Wan02, Conjecture IV], it was conjectured that a small perturbation of a birational isomorphism between Calabi-Yau manifolds is a sequence of ordinary flops. Hence the work above tells us that [Wan02, Conjecture IV] implies Conjecture 1.1. Kawamata in [Kaw08] showed that birational morphisms between Calabi-Yau manifolds can be decomposed into sequences of flops, however the structure of these flops in general is unknown.

In order to state our main theorem precisely, we need to set up some notation. Let $\widehat{\Phi}: X \rightarrow \widehat{X}$ be a birational isomorphism between Calabi-Yau manifolds. Fix a field $\mathbb{K}$ and fix Kähler forms $\omega_{X}, \omega_{\widehat{X}}$ on $X$ and $\widehat{X}$ respectively so that the de Rham cohomology classes $\left[\omega_{X}\right] \in H^{2}(X ; \mathbb{R}),\left[\omega_{\widehat{X}}\right] \in H^{2}(\widehat{X} ; \mathbb{R})$ lift to integral cohomology classes. Then a standard argument (see [Kaw02, Lemma 4.2] or Corollary 7.2) tells us that the map $\widehat{\Phi}$ gives us natural identifications $H^{2}(X ; \mathbb{R}) \cong H^{2}(\widehat{X} ; \mathbb{R})$ and $H_{2}(X ; \mathbb{Z}) \cong H_{2}(\widehat{X} ; \mathbb{Z})$. As a result, we will not distinguish between these groups. Therefore we can define the following Novikov rings: 


$$
\begin{aligned}
\Lambda_{\mathbb{K}}^{\omega_{X}, \omega_{\widehat{X}}}= & \left\{\sum_{i \in \mathbb{N}} b_{i} t^{a_{i}}: b_{i} \in \mathbb{K}, a_{i} \in H_{2}(X ; \mathbb{Z}), \quad \min \left(\omega_{X}\left(a_{i}\right), \omega_{\widehat{X}}\left(a_{i}\right)\right) \rightarrow \infty\right\}, \\
& \Lambda_{\mathbb{K}}^{\omega_{X}}=\left\{\sum_{i \in \mathbb{N}} b_{i} t^{a_{i}}: b_{i} \in \mathbb{K}, a_{i} \in H_{2}(X ; \mathbb{Z}), \quad \omega_{X}\left(a_{i}\right) \rightarrow \infty\right\}, \\
& \Lambda_{\mathbb{K}}^{\omega_{\widehat{X}}}=\left\{\sum_{i \in \mathbb{N}} b_{i} t^{a_{i}}: b_{i} \in \mathbb{K}, a_{i} \in H_{2}(X ; \mathbb{Z}), \quad \omega_{\widehat{X}}\left(a_{i}\right) \rightarrow \infty\right\} .
\end{aligned}
$$

Here, the first Novikov ring is the intersection of the other two. The aim of this paper is to prove the following theorem.

Theorem 1.2. Let $\widehat{\Phi}: X \rightarrow \widehat{X}$ be a birational equivalence between Calabi-Yau manifolds and let $\omega_{X}$ and $\omega_{\widehat{X}}$ be Kähler forms on $X$ and $\widehat{X}$ respectively whose cohomology classes lift to integer cohomology classes. Then there exists a graded $\Lambda_{\mathbb{K}}^{\omega_{X}, \omega_{\widehat{X}}}$-algebra $Z$ and algebra isomorphisms

$$
Z \otimes_{\Lambda_{\mathbb{K}}^{\omega_{X}, \omega_{\widehat{X}}}} \Lambda_{\mathbb{K}}^{\omega_{X}} \stackrel{\cong}{\longrightarrow} Q H^{*}\left(X ; \Lambda_{\mathbb{K}}^{\omega_{X}}\right), \quad Z \otimes_{\Lambda_{\mathbb{K}}^{\omega_{X}, \omega_{\widehat{X}}}} \Lambda_{\mathbb{K}}^{\omega_{\widehat{X}}} \stackrel{\cong}{\longrightarrow} Q H^{*}\left(X ; \Lambda_{\mathbb{K}}^{\omega_{\widehat{X}}}\right)
$$

over the Novikov rings $\Lambda_{\mathbb{K}}^{\omega_{X}}$ and $\Lambda_{\mathbb{K}}^{\omega_{\widehat{X}}}$ respectively where $Q H^{*}$ means small quantum cohomology.

Note that small quantum cohomology of a Calabi-Yau manifold can be defined over any field $\mathbb{K}$ (see [Rua96]). Previous theorems identifying quantum cohomology rings of birational Calabi-Yau manifolds were proven using a degeneration argument (along with many additional ideas, such as a quantum Leray-Hirsch theorem). We will prove our theorem using Hamiltonian Floer cohomology. This proof has the advantage that it works in greater generality and it explains in some sense why the result should hold. The downside, in comparison to previous results, is that the isomorphisms (1.3) are not explicit. In fact, the algebra $Z$ isn't explicit either. Also, the results [Rua99], [LLW16a], [LLW16b] and [LLQW16] identify actual enumerative invariants through analytic continuation dictated by the identification $H_{2}(X ; \mathbb{Z}) \cong H_{2}(\widehat{X} ; \mathbb{Z})$. It might be possible to use ideas from [Sei16] and [GPS15] to extend Theorem 1.2 above so that we can identify such enumerative invariants in some special cases. The results [LLW16a], [LLW16b] and [LLQW16] also identify big quantum cohomology rings, whereas we only identify small quantum cohomology rings. Finally, one may ask if higher genus invariants of birational Calabi-Yau's are related (see [ILLW12]). We currently do not know if the techniques in this paper will be useful in answering this question.

We will give a sketch of the proof of Theorem 1.2 in Subsection 1.2. The ideas of this proof were inspired by ongoing work of Borman and Sheridan and it uses a version of symplectic cohomology defined in [CFH95], [CO18], [Gro15], [Ven18] and [Var18]. As a corollary of the theorem above, we provide an alternative proof of the fact that birational Calabi-Yau manifolds have the same cohomology groups over any field.

Corollary 1.3. Let $X, \widehat{X}$ be birational Calabi-Yau manifolds. Then they have isomorphic cohomology groups over any field.

Proof. We wish to show that $H^{p}(X ; \mathbb{K}) \cong H^{p}(\widehat{X} ; \mathbb{K})$ for any field $\mathbb{K}$ and for each $p \in \mathbb{Z}$. Fix such a $\mathbb{K}$ and $p \in \mathbb{Z}$. Let $\check{\mathbb{F}}, \mathbb{F}, \widehat{\mathbb{F}}$ be the field of fractions of $\Lambda_{\mathbb{K}}^{\omega_{X}, \omega_{\widehat{X}}}, \Lambda_{\mathbb{K}}^{\omega_{X}}$ and $\Lambda_{\mathbb{K}}^{\omega_{\widehat{X}}}$ respectively. Then $\mathbb{K} \subset \check{\mathbb{F}}, \check{\mathbb{F}} \subset \mathbb{F}$ and $\check{\mathbb{F}} \subset \widehat{\mathbb{F}}$. Let $Z$ be as in Theorem 1.2 , define $\check{Z}:=Z \otimes_{\Lambda_{\mathbb{K}} \omega_{X}, \omega_{\widehat{X}}} \check{\mathbb{F}}$ and let 
$\check{Z}_{p}$ be the degree $p$ part of this graded algebra. By the universal coefficient theorem [Wei94, 3.6.1], we have the following isomorphisms of vector spaces:

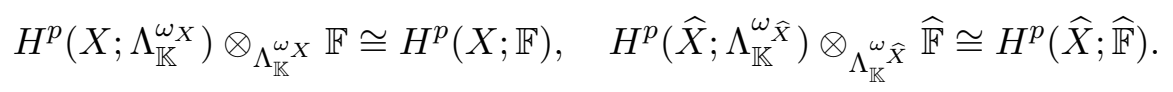

Hence by Equation (1.3), we have isomorphisms

$$
\check{Z}_{p} \otimes_{\check{\mathbb{F}}} \mathbb{F} \cong H^{p}(X ; \mathbb{F}), \quad \check{Z}_{p} \otimes_{\check{\mathbb{F}}} \widehat{\mathbb{F}} \cong H^{p}(\widehat{X} ; \widehat{\mathbb{F}}) .
$$

By the universal coefficient theorem, we also have isomorphisms

$$
H^{p}(X ; \mathbb{K}) \otimes_{\mathbb{K}} \mathbb{F} \cong H^{p}(X ; \mathbb{F}), \quad H^{p}(\widehat{X} ; \mathbb{K}) \otimes_{\mathbb{K}} \widehat{\mathbb{F}} \cong H^{p}(\widehat{X} ; \widehat{\mathbb{F}}) .
$$

Therefore by Equations (1.4) and (1.5), the dimension of $H^{p}(X ; \mathbb{K})$ is equal to the dimension of $H^{p}(\widehat{X} ; \mathbb{K})$. Hence $H^{p}(X ; \mathbb{K}) \cong H^{p}(\widehat{X} ; \mathbb{K})$.

1.1. Example. Even though we do not know what the isomorphisms (1.3) look like explicitly we will speculate what they should look like in a particular example when restricted to even degrees (see [Mor96, Section 4.3]). Suppose that $X, \widehat{X}$ are of dimension 3 and that there exists a class $\Gamma \in H_{2}(X ; \mathbb{Z})$ so that

- every connected one dimensional subvariety in $X$ representing $\Gamma$ or in $\widehat{X}$ representing $-\Gamma$ is isomorphic to $\mathbb{P}^{1}$ with normal bundle $\mathcal{O}(-1) \oplus \mathcal{O}(-1)$ and

- $X$ and $\widehat{X}$ are related by an Atiyah flop along all of these curves.

Since $H_{2}(X ; \mathbb{Z})$ is naturally identified with $H_{2}(\widehat{X} ; \mathbb{Z})$, we have by Poincaré duality a natural identification $H^{k}(X ; \mathbb{Q})=H^{k}(\widehat{X} ; \mathbb{Q})$ for each even $k \in \mathbb{Z}$. Hence from now on we will identify these cohomology groups. Let $\widehat{A}_{0}, \cdots, \widehat{A}_{l} \in H^{4}(X ; \mathbb{Q})$ be a basis so that $\widehat{A}_{0}$ is Poincaré dual to $\Gamma$ and let $A_{0}, \cdots, A_{l} \in H^{2}(X ; \mathbb{Q})$ be the dual basis with respect to the pairing $(\alpha, \beta) \rightarrow \int_{X} \alpha \cup \beta$. We speculate that the even degree part of the algebra $Z$ from Theorem 1.2 is isomorphic as a $\Lambda_{\mathbb{K}}^{\omega_{X}, \omega_{\widehat{X}}}$-module to $H^{\text {even }}\left(X ; \Lambda_{\mathbb{K}}^{\omega_{X}, \omega_{\widehat{X}}}\right)$ and the product $* Z$ on this module is uniquely determined by the structure constants:

$$
A_{i} *_{Z} A_{j}=A_{i} \cup_{X} A_{j}+l \delta_{0 i} \delta_{0 j} \widehat{A}_{0} t^{\Gamma}+\sum_{k=0}^{l} \sum_{\beta \notin \mathbb{Z} \Gamma} G W_{0,3}^{X, \beta}\left(A_{i}, A_{j}, A_{k}\right) \widehat{A}_{k} t^{\beta}, i, j \in\{0, \cdots, l\} .
$$

By replacing the class $A_{0}$ in (1.6) with $\frac{1}{1-t^{\Gamma}} A_{0}$ and $-\frac{t^{-\Gamma}}{1-t^{-\Gamma}} A_{0}$ respectively and the class $\widehat{A}_{0}$ with $\left(1-t^{\Gamma}\right) \widehat{A}_{0}$ and $-\frac{1-t^{-\Gamma}}{t^{-\Gamma}} \widehat{A}_{0}$ respectively, we get the isomorphisms in Equation (1.3).

1.2. Sketch of Proof. Theorem 1.2 is proven using Hamiltonian Floer cohomology. Very roughly, Hamiltonian Floer cohomology $H F^{*}(H)$ is a cohomology ring whose chain complex is generated by 1-periodic orbits of a Hamiltonian $H$. The key property of Hamiltonian Floer cohomology is that it is isomorphic to quantum cohomology and hence it is sufficient for us to show that the Hamiltonian Floer cohomology algebras of appropriate Hamiltonians on $\left(X, \omega_{X}\right)$ and $\left(\widehat{X}, \omega_{\widehat{X}}\right)$ respectively are related via equations similar to (1.3). In order to do this, we choose Zariski dense affine open subsets $A \subset X, \widehat{A} \subset \widehat{X}$ so that $\widehat{\Phi}$ maps $A$ isomorphically to $\widehat{A}$. The key idea now is to choose Hamiltonians $H$ on $X$ and $\widehat{H}$ on $\widehat{X}$ so that $\left.H\right|_{A}=\left.\widehat{\Phi}^{*} \widehat{H}\right|_{\widehat{A}}$ and which are constant outside a large compact subset $K$ of $A$. One also has to modify $\omega_{X}$ and $\omega_{\widehat{X}}$ so that these Kähler forms agree near $K$. If one could ignore all 1-periodic orbits of $H$ and $\widehat{H}$ outside $K$ then their Hamiltonian Floer groups would be 'identical' and hence we would be done. However it turns out that if one ignores these orbits, one gets groups that are no longer isomorphic to quantum cohomology. In order to get around 
this problem, we consider a sequence of such Hamiltonians tending to infinity outside $K$. We package all of this data into a group called symplectic cohomology and show that these groups are in fact isomorphic to quantum cohomology. In the following subsections, we provide a slightly more detailed sketch of the proof.

1.2.1. Hamiltonian Floer Cohomology with Alternative Filtrations. In this subsection, we summarize the results of Section 2. Let $(M, \omega)$ be a symplectic manifold with trivial first Chern class and fix an $\omega$-tame almost complex structure $J$. A contact cylinder is a codimension 0 symplectic embedding of a subset $\check{C}=[1-\epsilon, 1+\epsilon] \times C$ of a symplectization of a contact manifold $C$ which bounds a Liouville domain $D$ (see Definition 2.3). Let $H$ be a time dependent Hamiltonian on $M$ which is compatible with this contact cylinder (see Definition 2.4) whose 1-periodic orbits are non-degenerate. A capped 1-periodic orbit of $H$ is a 1-periodic orbit $\gamma$ together with a certain equivalence class of surfaces $\widetilde{\gamma}: \Sigma \longrightarrow M$ with boundary equal to $\gamma$ (see Definition 2.1). Now to each capped 1-periodic orbit $\gamma$ and to each closed 2-form $\widetilde{\omega}$ which is 'compatible' with $\check{C}$ and $J$ we can assign an action. This action depends on $\gamma, H$ and the cohomology class of $\widetilde{\omega}$ together with two additional parameters (Corollary 2.8 and Remark 2.9). Therefore, one can think of the action of $\gamma$ as a particular function $\mathcal{A}_{\check{C}, H}(\gamma)$ from (a certain subset $Q_{\check{C}}$ of) $H^{2}(M, D ; \mathbb{R}) \times \mathbb{R} \times \mathbb{R}$ to $\mathbb{R}$. Morally, one should think of $D$ as the 'complement' of a particular ample divisor and that the action function $\mathcal{A}_{\check{C} . H}(\gamma)$ records the usual action together with the intersection numbers of the capping surface $\widetilde{\gamma}$ with various components of this divisor. Let $a_{ \pm}: Q_{ \pm} \longrightarrow \mathbb{R}$ be continuous functions where $Q_{ \pm} \subset Q_{\check{C}}$ are certain cones in $Q_{\check{C}}$. We define the chain complex $C F_{\check{C}, a_{-}, a_{+}}^{*}(H)$ to be the free abelian group generated by capped 1-periodic orbits $\gamma$ satisfying $a_{-} \leq\left.\mathcal{A}_{\check{C}, H}(\gamma)\right|_{Q_{-}}$and $a_{+} \not \leq\left.\mathcal{A}_{\check{C}, H}(\gamma)\right|_{Q_{+}}$ (see Definition 2.59 for more details). The differential is a matrix with respect to the above basis of capped 1-periodic orbits whose entries 'count' solutions to a particular PDE with boundary conditions given by these orbits (Definition 2.63). We define the Hamiltonian Floer

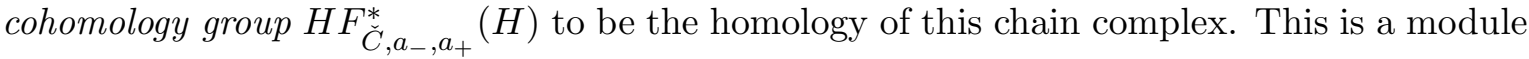
over a particular Novikov ring $\Lambda_{\mathbb{K}}^{Q_{+},+}$(Definition 2.56). Here are some key examples.

$\{$ A $\}$ The case when $\check{C}$ is the empty set and $Q_{-}=Q_{+}$are 1-dimensional cones spanned by $([\omega], 1,1) \in H^{2}(M ; \mathbb{R}) \times \mathbb{R}^{2}$. In this case, one gets the usual Hamiltonian Floer groups and the usual action filtration (used in, say, [FH94]). The Novikov ring $\Lambda_{\mathbb{K}}^{Q_{+},+}$ in this case is equal to (1.2) where $\omega_{X}$ is replaced by $\omega$ and where only non-negative exponents are allowed (I.e. $\omega\left(a_{i}\right) \geq 0$ ).

$\{\mathrm{B}\}$ The case when $\check{C}$ is non-empty, $Q_{+}$is a one dimensional cone and $Q_{-}$is a two dimensional cone containing $Q_{+}$which projects to the cone spanned by $[\omega] \in H^{2}(M ; \mathbb{R})$. In this case the corresponding Floer cohomology group is defined over the same Novikov ring, but since $Q_{-}$is larger, one can use it to ignore certain 1-periodic orbits.

$\{\mathrm{C}\}$ Finally there is the case when $Q_{+}$is a certain two dimensional cone and $Q_{-}$is a four dimensional cone. The Novikov ring in this case is a subring of (1.1) where only non-negative exponents are allowed (I.e. $\omega_{X}\left(a_{i}\right), \omega_{\widehat{X}}\left(a_{i}\right) \geq 0$ ). We will use this case to define the algebra $Z$.

These Hamiltonian Floer groups satisfy the following properties:

(HF1) (Definition 2.69). If $H_{-} \leq H_{+}$(plus some other conditions) then there is a natural continuation map $\Phi_{H^{-}, H^{+}}^{p}: H F_{\check{C}, a_{-}, a_{+}}^{p}\left(H^{-}\right) \longrightarrow H F_{\check{C}, a_{-}, a_{+}}^{p}\left(H^{+}\right)$which is functorial. 
(HF2) (Definition 2.77). If $Q_{ \pm}^{1} \subset Q_{ \pm}^{0}$ and $a_{ \pm}^{1} \leq\left. a_{ \pm}^{0}\right|_{Q_{ \pm}^{1}}$ then there is a natural action map

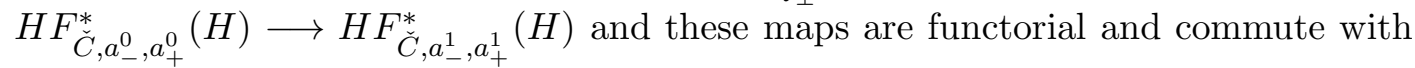
continuation maps.

(HF3) (Definition 2.85). Suppose

- $\left(H^{j}\right)_{j=0,1,2}$ are Hamiltonians satisfying $H^{0}, H^{1}<\frac{1}{2} H^{2}$,

- $\left(Q_{ \pm}^{j}\right)_{j=0,1,2}$ are certain cones in $H^{2}(M, D ; \mathbb{R}) \times \mathbb{R} \times \mathbb{R}$ satisfying $Q_{ \pm}^{2} \subset Q_{ \pm}^{j}$ and

- $a_{ \pm}^{j}: Q_{ \pm}^{j} \longrightarrow \mathbb{R}$ are certain continuous functions satisfying $a_{-}^{2} \leq a_{-}^{0}+a_{-}^{1}$ and $a_{+}^{2} \leq \min \left(a_{+}^{0}+a_{-}^{1}, a_{-}^{0}+a_{+}^{1}\right)$.

Then there is a pair of pants product map $H F_{\check{C}, a_{-}^{0}, a_{+}^{0}}^{p_{0}}\left(H^{0}\right) \otimes_{\Lambda_{\mathbb{K}}^{Q,+}} H F_{\check{C}, a_{-}^{1}, a_{+}^{1}}^{p_{1}}\left(H^{1}\right) \longrightarrow$ $H F_{\tilde{C}, a_{-}^{2}, a_{+}^{2}}^{p_{0}+p_{1}}\left(H^{2}\right)$ commuting with all of the maps above.

1.2.2. Lower semi-continuous Hamiltonians. The following subsection summarizes Section 3. A lower semi-continuous Hamiltonian is just a function $S^{1} \times M \longrightarrow \mathbb{R} \cup\{\infty\}$ which is lower semi-continuous. The good thing about this condition is that the set of smooth Hamiltonians smaller than $H$ form a directed system with respect to the usual ordering $\leq$. For a lower semi-continuous Hamiltonian $H$ compatible with a contact cylinder $\check{C}$, we can define $H F_{\check{C}, a_{-}, a_{+}}^{*}(H)$ to be the direct limit of $H F_{\check{C}, a_{-}, a_{+}}^{*}(\check{H})$ for all smooth $\check{C}$ compatible Hamiltonians smaller than $H$. These satisfy the same properties (HF1)-(HF3) above.

1.2.3. Symplectic Cohomology. The problem with the Floer groups above is that they do not have the correct invariance properties and they are not algebras. We resolve these issues in Sections 4 and 5 . Let $M, \check{C}, D$ be as above. Let $Q_{-} \subset Q_{+}$be two cones in $Q_{\check{C}}$. For a closed set $K \subset D$ we define the symplectic cohomology algebra

$$
S H_{\breve{C}, Q_{-}, Q_{+}}^{*}(K \subset M):=\underset{a_{-}}{\lim } \underset{a_{+}}{\lim } H F_{\check{C}, a_{-}, a_{+}}^{*}\left(H_{K}\right)
$$

where we are using the directed system of continuous functions $a_{ \pm}: Q_{ \pm} \longrightarrow \mathbb{R}$ with the usual ordering $\leq$ for $a_{+}$and the opposite ordering for $a_{-}$and where $H_{K}$ is the lower-semi-continuous Hamiltonian

$$
H_{K}: M \longrightarrow \mathbb{R} \cup\{\infty\}, \quad H_{K}(x):= \begin{cases}0 & \text { if } x \in K \\ \infty & \text { otherwise }\end{cases}
$$

This is defined over a particular Novikov ring $\Lambda_{\mathbb{K}}^{Q_{+}}$and has a product induced by the pair of pants product. The papers [CFH95], [CO18], [Gro15], [Ven18] and [Var18] have a similar definition of symplectic cohomology. However there are slight differences between all of these definitions (which potentially could lead to different algebras). One main difference is that some of the definitions above involve building a chain complex first, and then taking homology. Our definition does not do this, but only for the sake of ease. The symplectic cohomology algebra satisfies the following properties (see the cited definitions and Propositions/Theorems for more accurate statements):

(SH1) (Definition 4.3). If $K_{+} \subset K_{-} \subset D$ are closed subsets then we have a transfer map $S H_{\breve{C}, Q_{-}, Q_{+}}^{*}\left(K_{-} \subset M\right) \longrightarrow S H_{\breve{C}, Q_{-}, Q_{+}}^{*}\left(K_{+} \subset M\right)$ which is functorial.

(SH2) (Definition 4.4). If $Q_{ \pm}^{1} \subset Q_{ \pm}^{0}$ then there is an action map $S H_{\breve{C}, Q_{-}^{0}, Q_{+}^{0}}^{*}(K \subset M) \longrightarrow$ $S H_{\breve{C}, Q_{-}^{1}, Q_{+}^{1}}^{*}(K \subset M)$. These maps commute with continuation maps and are functorial. 
(SH3) (Theorem 5.10). If $K=M$ and $\check{C}, Q_{ \pm}$are as in $\{\mathrm{A}\}$ then $S H_{\check{C}, Q_{-}, Q_{+}}^{*}(K \subset M)$ is isomorphic to quantum cohomology. Also the 'derived' version of symplectic cohomology $\lim _{\longrightarrow a_{-}} \lim _{a_{+}}^{1} H F_{\tilde{C}, a_{-}, a_{+}}^{*}\left(H_{K}\right)$ vanishes.

(SH4) (Theorem 5.12). If the complement $M-K$ is stably displaceable (I.e. $(M-K) \times S^{1} \subset$ $M \times T^{*} S^{1}$ is displaceable by a Hamiltonian symplectomorphism) and $\breve{C}, Q_{ \pm}$are as in $\{\mathrm{A}\}$ then the transfer map $S H_{\emptyset, Q_{-}, Q_{+}}^{*}(M \subset M) \longrightarrow S H_{\emptyset, Q_{-}, Q_{+}}^{*}(K \subset M)$ is an isomorphism.

(SH5) (Proposition 5.24). If $\check{C}, Q_{-}, Q_{+}$are as in $\{\mathrm{B}\}$ and the Liouville domain $D$ is index bounded (Definition 5.23) then the action map

$$
S H_{\check{C}, Q_{-}, Q_{+}}^{*}(D \subset M) \longrightarrow S H_{\check{C}, Q_{+}, Q_{+}}^{*}(D \subset M)
$$

is an isomorphism.

(SH6) (Proposition 5.32). Suppose that $\check{C}_{0}, \check{C}_{1}$ are index bounded contact cylinders with associated Liouville domains $D_{0}$ and $D_{1}$ respectively satisfying $D_{1} \subset D_{0}$ along with some other conditions (essentially $D_{0}$ and $D_{1}$ need to be 'large' in some sense). Then the transfer map $S H_{\check{C}, Q_{-}, Q_{+}}^{*}\left(D_{0} \subset M\right) \longrightarrow S H_{\check{C}, Q_{-}, Q_{+}}^{*}\left(D_{1} \subset M\right)$ is an isomorphism where $\left(Q_{-}, Q_{+}\right)$is as in $\{\mathrm{B}\}$.

(SH7) (Theorem 5.39 and Proposition 11.2). Let $\check{C}$ be an index bounded contact cylinder with associated Liouville domain $D$. Suppose we have inclusions of rational polyhedral cones $Q_{ \pm}^{1} \subset Q_{ \pm}^{0}$ where $Q_{-}^{1}$ has dimension at least 2 (e.g. case $\{\mathrm{B}\}$ or $\{\mathrm{C}\}$ ). Also suppose $\lim _{\longrightarrow} a_{-} \lim _{a_{+}}^{1} H F_{\breve{C}, a_{-}, a_{+}}^{*}\left(H_{D}\right)=0$ where $H_{D}$ is defined in (1.7). Then we have an isomorphism

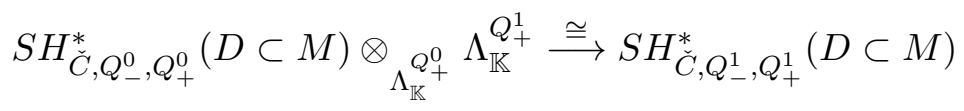

induced by the action map.

(SH8) (Proposition 2.19 and Lemma 8.3). Let $\check{C}$ be a contact cylinder with associated Liouville domain $D$. Suppose $V \subset M-D-\check{C}$ is a union of real codimension $\geq 4$ submanifolds. Let $Q_{ \pm}$be cones so that $Q_{-}$is of dimension $\geq 2$. Then the Floer trajectories and orbits defining $S H_{\overparen{C}, Q_{-}, Q_{+}}^{*}(D \subset M)$ can be made to avoid $V$. Hence this group only depends on these structures restricted to $M-V$.

1.2.4. Sketch of Proof of Main Theorem 1.2. Here we summarize the ideas behind the proof of Theorem 1.2 coming from Sections 6 and 7. The proof has two parts. In part (1), we modify the symplectic forms on $X$ and $\widehat{X}$ so that they agree on a certain large open subset and so that they admit certain index bounded contact cylinders. In part (2), we use properties (SH1)-(SH8) to finish our proof.

Part (1): First of all, we choose Zariski dense affine subvarieties $A \subset X, \widehat{A} \subset \widehat{X}$ so that

(1) the birational morphism $\widehat{\Phi}$ induces an isomorphism $\Phi: A \longrightarrow \widehat{A}$ and

(2) $\omega_{X}$ and $\omega_{\widehat{X}}$ come from effective ample divisors with support equal to $X-A$ and $\widehat{X}-\widehat{A}$ respectively (after rescaling these forms).

By Corollary 6.20, we have that $X-A$ is stably displaceable (by an $h$-principle). Hence there is a compact subset $K \subset A$ so that $X-K$ is stably displaceable. By Proposition 6.7 we can construct an index bounded contact cylinder $\check{C}$ on $X$ whose associated Liouville domain $D$ contains $K$ (here we use the fact that $\widehat{X}$ is Calabi-Yau). By using the ample divisors above, we can modify the Kähler form $\omega_{\widehat{X}}$ (without changing its cohomology class up to rescaling) 
so that $\omega_{X}$ and $\Phi^{*} \omega_{\widehat{X}}$ agree near $D$. Again, by Corollary 6.20 we can find a compact subset $\widehat{K} \subset \widehat{A}$ containing $\Phi(D)$ so that $\widehat{X}-\widehat{K}$ is stably displaceable. Also by Proposition 6.7 one can construct an index bounded contact cylinder $\widehat{C}$ in $\widehat{A}$ whose associated Liouville domain $\widehat{D} \subset \widehat{A}$ contains $\widehat{K}$.

Part (2): From now on we identify $H^{2}(X, D ; \mathbb{R})=H^{2}(\widehat{X}, \widehat{D} ; \mathbb{R})$. We let $Q_{\omega_{X}}$ and $Q_{\omega_{\widehat{X}}}$ be 1-dimension cones spanned by $\left(\left[\omega_{X}\right], 1,1\right)$ and $\left(\left[\omega_{\widehat{X}}\right], 1,1\right)$ respectively as in $\{\mathrm{A}\}$. We let $Q_{ \pm}$, $\widehat{Q}_{ \pm}$be the corresponding enlarged cones as in $\{\mathrm{B}\}$. Finally we let $\widetilde{Q}_{ \pm}$be the cones spanned by both $Q_{ \pm}$and $\widehat{Q}_{ \pm}$(these are cones as in $\{\mathrm{C}\}$ ). By (SH3), (SH4) and (SH5) we have that $S H_{\overparen{C}, Q_{-}, Q_{+}}^{*}(D \subset X)$ and $S H_{\widehat{C}, \widehat{Q}_{-}, \widehat{Q}_{+}}^{*}(\widehat{D} \subset \widehat{X})$ are isomorphic to the quantum cohomology rings of $X$ and $\widehat{X}$ respectively. By $(\mathrm{SH} 6)$ we have that the transfer map $S H_{\widehat{C}, \widehat{Q}_{-}, \widehat{Q}_{+}}(\widehat{D} \subset$ $\widehat{X}) \longrightarrow S H_{\Phi(\check{C}), \widehat{Q}_{-}, \widehat{Q}_{+}}^{*}(\Phi(D) \subset \widehat{X})$ is an isomorphism. Define the $\Lambda_{\mathbb{K}}^{\omega_{X}, \omega_{\widehat{X}}}$-algebra $Z:=$ $S H_{\breve{C}_{,} \widetilde{Q}_{-}, \widetilde{Q}_{+}}(D \subset X)$. Now since the regions $V_{X} \subset X$ and $V_{\widehat{X}} \subset \widehat{X}$ for which the birational morphisms $\widehat{\Phi}$ and $\widehat{\Phi}^{-1}$ are ill defined has real codimension $\geq 4$ by Lemma 7.1, we have by (SH8) an isomorphism of $\Lambda_{\mathbb{K}}^{\omega_{X}, \omega_{\hat{X}}}$-algebras

$$
Z \cong S H_{\widetilde{C}, \widetilde{Q}_{-}, \widetilde{Q}_{+}}^{*}(\Phi(D) \subset \widehat{X}) .
$$

The isomorphisms (1.3) now follow from Equation (1.8) combined with the second part of (SH3) and (SH7).

\subsection{Notation Throughout this paper.}

- We will fix a ring $\mathbb{K}$.

- $(M, \omega)$ will be a compact connected symplectic manifold of dimension $2 n$ satisfying $c_{1}(\omega)=0$ and where $[\omega] \in H^{2}(M ; \mathbb{R})$ lifts to an integral cohomology class,

- $J_{0}$ is a fixed almost complex structure on $M$ taming $\omega$,

- $\left(V_{i}\right)_{i=1}^{l}$ is a finite collection of (not necessarily properly embedded) submanifolds of $M$ of codimension $\geq 4$ and where $V:=\cup_{i=1}^{l} V_{i}$ is compact,

- $\mathbb{T}:=\mathbb{R} / \mathbb{Z}, \mathbb{I}_{-}:=(-\infty, 0], \mathbb{I}_{+}:=[0, \infty)$,

- $(s, t)$ will be the natural coordinate system on $\mathbb{I}_{ \pm} \times \mathbb{T}$ or $\mathbb{R} \times \mathbb{T}$.

- For any manifold $\Sigma, g^{\Sigma}\left(J_{0}, V, \omega\right)$ is the space of smooth families of $\omega$-tame almost complex structures $J:=\left(J_{\sigma}\right)_{\sigma \in \Sigma}$ smoothly parameterized by $\Sigma$ equipped with the $C^{\infty}$ topology so that all the derivatives of $J$ and $J_{0}$ agree at $v$ for all $v \in V$.

- $\mathcal{J}\left(J_{0}, V, \omega\right):=\mathcal{J}^{\mathrm{pt}}\left(J_{0}, V, \omega\right)$.

- If $I$ is a set and $W$ is a vector space then $W^{I}$ is the vector space of maps $I \longrightarrow W$ or equivalently tuples $\left(w_{j}\right)_{j \in I}$ of elements in $W$.

Acknowledgments: I would like to thank Nick Sheridan for extremely helpful conversations concerning Novikov parameters, Strom Borman for helping me with transfer map properties in Section 5.6 and Umut Varolgunes for many helpful suggestions and corrections. I would also like to thank Paul Seidel for his encouragement and suggestions for future work and Yongbin Ruan and Y.P. Lee for their helpful comments. Finally I would like to thank Chenyang $\mathrm{Xu}$ and Qizheng Yin for helpful discussions. This paper is supported by the NSF grants DMS-1508207 and DMS-1811861.

\section{Hamiltonian Floer Cohomology and Filtrations}

\subsection{Alternative Action Values of Periodic Orbits.}


Definition 2.1. A Hamiltonian is a smooth family of functions $H=\left(H_{t}\right)_{t \in \mathbb{T}}$ on a symplectic manifold (by default this is $(M, \omega)$ unless stated otherwise). It is autonomous if $H_{t}$ does not depend on $t$, and hence we usually express such a Hamiltonian as a single function $M \longrightarrow \mathbb{R}$. The time $t$ flow $\left(\phi_{t}^{H}: M \longrightarrow M\right)_{t \in \mathbb{R}}$ of a Hamiltonian $H \equiv\left(H_{t}\right)_{t \in \mathbb{T}}$ is the time $t$ flow of the unique time dependent vector field $\left(X_{t}^{H}\right)_{t \in \mathbb{R}}$ satisfying $i_{X_{t}^{H}} \omega=-d H_{t}$ for all $t \in \mathbb{R}$. A 1-periodic orbit is a smooth map $\gamma: \mathbb{T} \longrightarrow M$ satisfying $\dot{\gamma}=X_{t}^{H}$ for all $t \in \mathbb{T}$. A 1-periodic orbit $\gamma$ is non-degenerate if the linearized return map $D \phi_{1}^{H}: T_{\gamma(0)} M \longrightarrow T_{\gamma(0)} M$ has no eigenvalue equal to 1 . A capped loop is an equivalence class of pairs $(\widetilde{\gamma}, \check{\gamma})$ of smooth maps

$$
\widetilde{\gamma}: S \longrightarrow M, \quad \check{\gamma}: \mathbb{T} \longrightarrow \partial S
$$

where $S$ is a smooth oriented surface with boundary, $\check{\gamma}$ is an orientation preserving diffeomorphism and where any two such pairs $\left(\widetilde{\gamma}_{0}, \check{\gamma}_{0}\right),\left(\widetilde{\gamma}_{1}, \check{\gamma}_{1}\right)$ are equivalent if $\widetilde{\gamma}_{0} \circ \check{\gamma}_{0}=\widetilde{\gamma}_{1} \circ \check{\gamma}_{1}$ and if the surface obtained by gluing $\widetilde{\gamma}_{0}$ and $\widetilde{\gamma}_{1}$ along the boundary via the map $\check{\gamma}_{0} \circ \check{\gamma}_{1}^{-1}$ is null homologous. More precisely, this gluing is defined to be the continuous map

$$
\widetilde{\gamma}_{0} \star \widetilde{\gamma}_{1}: S_{0} \sqcup \bar{S}_{1} / \sim \longrightarrow, \quad \gamma_{0} \star \gamma_{1}(\sigma):= \begin{cases}\widetilde{\gamma}_{0} & \text { if } z \in S_{0} \\ \widetilde{\gamma}_{1} & \text { otherwise }\end{cases}
$$

where $S_{0}$ is the domain of $\widetilde{\gamma}_{0}, \bar{S}_{1}$ is the domain of $\widetilde{\gamma}_{1}$ with the opposite orientation and where the identification $\sim$ is defined to be

$$
\partial S_{0} \ni \check{\gamma}_{0}(t) \sim \check{\gamma}_{1}(t) \in \partial S_{1}, t \in \mathbb{T} .
$$

If $(\widetilde{\gamma}, \check{\gamma})$ is a capped loop, then the associated loop of $(\tilde{\gamma}, \check{\gamma})$ is the map $\widetilde{\gamma} \circ \check{\gamma}: \mathbb{T} \longrightarrow M$. We define $\widetilde{\mathcal{L}}(M)$ to be the space of capped loops equipped with the quotient topology induced from the $C^{\infty}$ topology on the space of pairs of maps as in Equation (2.1).

A capped 1-periodic orbit $\gamma$ of a Hamiltonian $H$ is a capped loop, whose associated loop $\bar{\gamma}: \mathbb{T} \longrightarrow M$ is a 1-periodic orbit of $H$. We call $\bar{\gamma}$ the associated 1-periodic orbit of $\gamma$. A capped 1 -periodic orbit $\gamma$ is non-degenerate if the associated 1-periodic orbit is non-degenerate.

Definition 2.2. Let $K=\left(K_{\sigma}\right)_{\sigma \in \Sigma}$ be a smooth family of functions on $M$ parameterized by a manifold $\Sigma$. Let $\widetilde{\omega}$ be a closed 2 -form on $M-V$. We say that $K$ is $\widetilde{\omega}$-compatible if there is a smooth family of functions $G=\left(G_{\sigma}\right)_{\sigma \in \Sigma}$ on $M-V$ so that

$$
i_{X_{K_{\sigma}}} \widetilde{\omega}=-d G_{\sigma}, \quad \forall \sigma \in \Sigma .
$$

We will call $G$ a primitive associated to $(K, \widetilde{\omega})$.

Now let $H:=\left(H_{t}\right)_{t \in \mathbb{T}}$ be a Hamiltonian which is $\widetilde{\omega}$-compatible and $F=\left(F_{t}\right)_{t \in \mathbb{T}}$ a primitive associated to $(H, \widetilde{\omega})$. The $(H, \widetilde{\omega}, F)$-action of a capped loop $\gamma:=(\widetilde{\gamma}, \check{\gamma})$ on $M$ where the associated loop $\bar{\gamma}: \mathbb{T} \longrightarrow M$ is disjoint from $V$ is defined to be

$$
\mathcal{A}_{H, \widetilde{\omega}, F}(\gamma):=-\int_{S}\left(\widetilde{\gamma}^{\prime}\right)^{*} \widetilde{\omega}+\int_{0}^{1} F_{t}(\bar{\gamma}(t)) d t
$$

where $\widetilde{\gamma}^{\prime}: S \longrightarrow M$ is some $C^{\infty}$ small perturbation of $\widetilde{\gamma}$ away from $\partial S$ so that its image is disjoint from $V$. If $\widetilde{\omega}$ extends to a smooth 2 -form on $M$ and $F$ extends to a smooth family of functions on $M$ then we define the $(H, \widetilde{\omega}, F)$-action of any capped loop $\gamma$ by Equation (2.4) with $\widetilde{\gamma}^{\prime}$ replaced by $\gamma$. If $(\widetilde{\gamma}, \check{\gamma})$ is any capped loop whose associated loop is constant then we define the $(H, \widetilde{\omega}, F)$-action $\mathcal{A}_{H, \widetilde{\omega}, F}(\gamma)$ to be $\mathcal{A}_{H, \widetilde{\omega}, F}\left(\gamma^{\prime}\right)$ where $\gamma^{\prime}$ is a capped loop disjoint from $V$ which is smoothly isotopic to $\gamma$ through capped loops with constant associated loops.

Note that the perturbations $\widetilde{\gamma}^{\prime}$ and $\gamma^{\prime}$ above exist since $V$ is a finite union of codimension $\geq 4$ submanifolds of $M$. Note also that such an action will usually be computed for capped 1-periodic orbits of $H$. 
We will only deal with very specific closed 2 -forms $\widetilde{\omega}$ associated to certain contact hypersurfaces inside $(M, \omega)$. We will now introduce such closed 2 -forms.

Definition 2.3. (See Figure 1) A contact cylinder in $M$ consists of a codimension 0 submanifold

$$
\check{C}:=[1-\epsilon, 1+\epsilon] \times C \subset M-V
$$

so that $\left.\omega\right|_{\check{C}}=d\left(r_{C} \alpha_{C}\right)$ where $r_{C}: \check{C} \longrightarrow[1-\epsilon, 1+\epsilon]$ is the natural projection map, and $\alpha_{C}$ is a contact form on $C$. We also require that $\{0\} \times C$ is the boundary of a compact codimension 0 submanifold $D$ so that $\left.\omega\right|_{D}=d \theta$ for some $\theta \in \Omega^{1}(D)$ satisfying $\left.\theta\right|_{D \cap \check{C}}=r_{C} \alpha_{C}$. Here $r_{C}$ is called the radial coordinate associated to $\check{C}, \alpha_{C}$ is called the contact form associated to $\check{C}$ and $D$ is called the Liouville domain associated to $\check{C}$. A 2-form $\widetilde{\omega} \in \Omega^{2}(M-V)$ is $\check{C}$-compatible if

(a) $\widetilde{\omega}$ is closed and $J_{0}$-tame outside $D \cup \check{C}$,

(b) $\left.\widetilde{\omega}\right|_{\check{C}}=d\left(f_{\widetilde{\omega}}\left(r_{C}\right) r_{C} \alpha\right)$ where $f_{\widetilde{\omega}}: \mathbb{R} \longrightarrow \mathbb{R}$ is a smooth function satisfying:

$$
\left.f_{\widetilde{\omega}}\right|_{(-\infty, 1+\epsilon / 4]}=\lambda_{\widetilde{\omega}}^{-},\left.\quad f_{\widetilde{\omega}}\right|_{[1+\epsilon / 2, \infty)}=\lambda_{\widetilde{\omega}}^{+}, \quad f_{\widetilde{\omega}}^{\prime} \geq 0 .
$$

for some constants $\lambda_{\widetilde{\omega}}^{ \pm} \geq 0$,

(c) $\left.\widetilde{\omega}\right|_{D}=\lambda_{\widetilde{\omega}}^{-} \omega$ and

(d) $\widetilde{\omega}=\lambda_{\widetilde{\omega}}^{+} \omega$ if $\check{C}$ is the empty set.

We call $\lambda_{\widetilde{\omega}}^{ \pm}$the scaling constants for $\widetilde{\omega}$ and $f_{\widetilde{\omega}}$ the scaling function for $\widetilde{\omega}$. A family of 2 -forms $\widetilde{\omega}^{\bullet}=\left(\widetilde{\omega}^{\sigma}\right)_{\sigma \in \Sigma}$ is $\check{C}$-compatible if $\widetilde{\omega}_{\sigma}$ is $\check{C}$-compatible for each $\sigma \in \Sigma$. If $\check{C}$ is the empty contact cylinder then $\lambda_{\widetilde{\omega}}^{-}$is defined to be arbitrary (I.e. we can choose $\lambda_{\widetilde{\omega}}^{-}$to be anything we like and it is considered as part of the data defining $\widetilde{\omega})$.

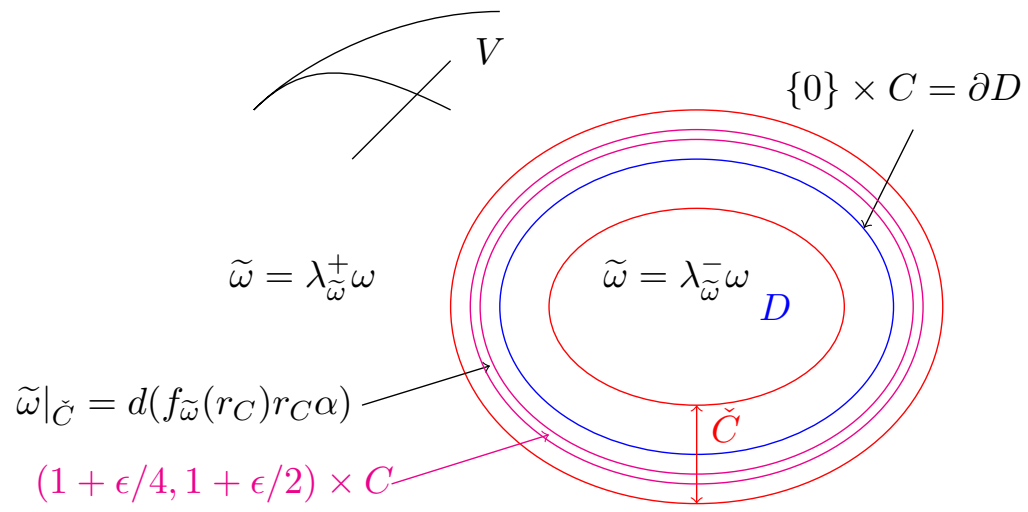

Figure 1. A contact cylinder

Definition 2.4. An autonomous Hamiltonian $K: M \longrightarrow \mathbb{R}$ is weakly $\check{C}$-compatible if $\left.K\right|_{[1+\epsilon / 8,1+\epsilon / 2] \times C}=\lambda_{K} r_{C}+m_{K}$ for some constants $\lambda_{K}$ and $m_{K}$. The constant $\lambda_{K}$ is called the slope of $K$ along $\check{C}$ and $m_{K}$ is called the height of $K$ at $\check{C}$. Also if $\check{C}$ is the empty contact cylinder then we define the slope and height of $K$ to be 0 . We say that $K$ is $\check{C}$-compatible if it is weakly $\check{C}$-compatible and if $\left.K\right|_{M-(D \cup \check{C})}$ is constant. A smooth family of autonomous Hamiltonians $K_{\bullet}:=\left(K_{\sigma}\right)_{\sigma \in \Sigma}$ parameterized by a manifold $\Sigma$ is (weakly) $\check{C}$-compatible if $K_{\sigma}$ is (weakly) $\check{C}$-compatible for each $\sigma \in \Sigma$. An almost complex structure $J$ on $M$ is $\check{C}$-compatible 
if $J \in \mathcal{J}\left(J_{0}, V, \omega\right)$, and $d r_{C} \circ J=-\alpha_{C}$ inside $[1+\epsilon / 8,1+\epsilon / 2] \times C$. A smooth family of almost complex structures $J_{\bullet}=\left(J_{\sigma}\right)_{\sigma \in \Sigma}$ is $\check{C}$-compatible if $J_{\sigma}$ is $\check{C}$-compatible for each $\sigma \in \Sigma$.

Remark 2.5. The space of 2 -forms which are $\check{C}$-compatible is weakly contractible (in fact it forms a convex subset of $\Omega^{2}(M)$ ). Also the space of (weakly) $\check{C}$-compatible Hamiltonians (resp. almost complex structures) is weakly contractible.

By a direct calculation, we have the following lemma and corollary:

Lemma 2.6. Let

- $\check{C}$ be a contact cylinder with cylindrical coordinate $r_{C}$ and associated Liouville domain $D$,

- $\widetilde{\omega}$ be a 2-form compatible with $\check{C}$ where $\lambda_{\widetilde{\omega}}^{ \pm}$(resp. $f_{\widetilde{\omega}}$ ) are the scaling constants (resp. scaling function) for $\widetilde{\omega}$ and

- let $H=\left(H_{t}\right)_{t \in \mathbb{T}}$ be a weakly $\check{C}$-compatible Hamiltonian which is $\check{C}$-compatible if $\widetilde{\omega}$ is not a locally constant multiple of $\omega$ outside $[1+\epsilon / 4,1+\epsilon / 2] \times C$ and let $\left(\lambda_{H_{t}}\right)_{t \in \mathbb{T}}$ and $\left(m_{H_{t}}\right)_{t \in \mathbb{T}}$ be the slopes and heights of $\left(H_{t}\right)_{t \in \mathbb{T}}$ along $\check{C}$ respectively.

Then the smooth family of functions $F^{H, \breve{C}, \widetilde{\omega}}:=\left(F^{H_{t}, \breve{C}, \widetilde{\omega}}\right)_{t \in \mathbb{T}}$ defined by

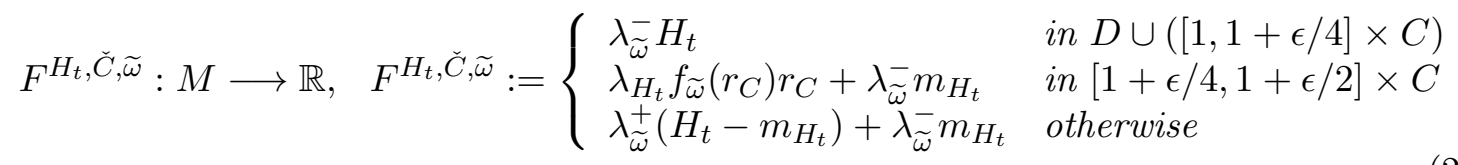

is a primitive associated to $(H, \widetilde{\omega})$ as in Definition 2.2 for all $t \in \mathbb{T}$ (See Figure 2).

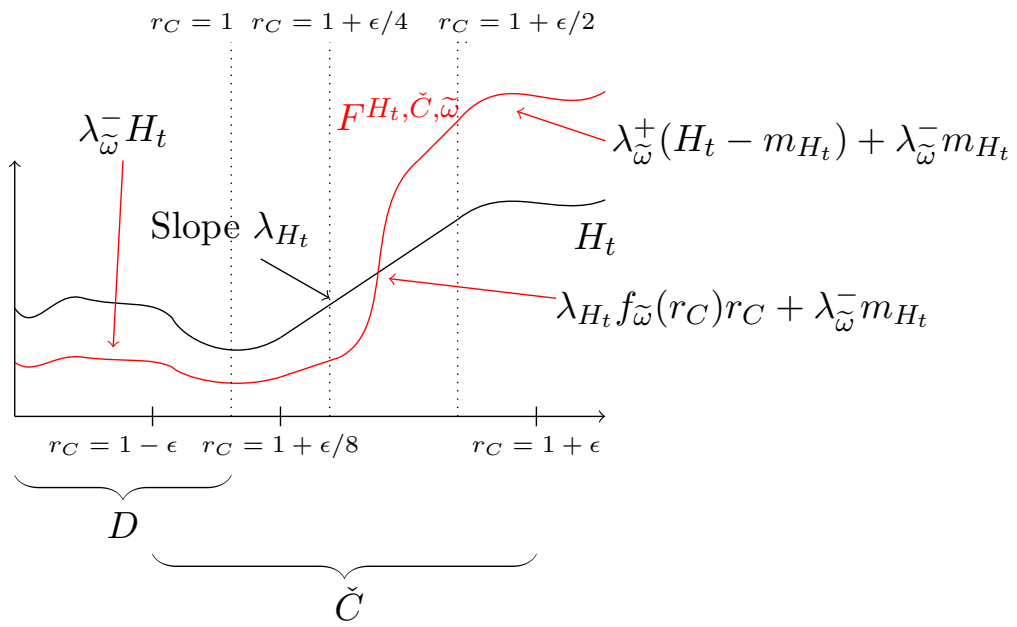

Figure 2. Primitive associated to $(H, \widetilde{\omega})$ 
Remark 2.7. Most of the important Hamiltonians in subsections 5.5, 5.6 and 5.7 will have small derivatives outside $D \cup([1,1+\epsilon / 8] \times C)$. This makes our calculations easier. It is good to keep such Hamiltonians in mind throughout this paper since they appear in many of the most important calculations. In this special case, Figure 2 might look like Figure 3 instead.

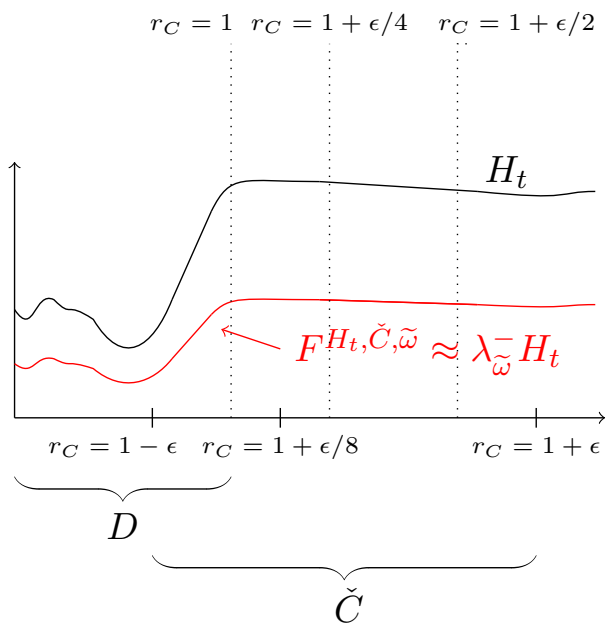

Figure 3 . Primitive associated to $(H, \widetilde{\omega})$, where $H$ has small derivatives outside $D \cup([1,1+\epsilon / 8] \times C)$.

We have the following corollary of Lemma 2.6.

Corollary 2.8. Let $\check{C}, \widetilde{\omega}, H=\left(H_{t}\right)_{t \in \mathbb{T}}, \lambda_{\widetilde{\omega}}^{ \pm},\left(\lambda_{H_{t}}\right)_{t \in \mathbb{T}},\left(m_{H_{t}}\right)_{t \in \mathbb{T}}, F^{H, \check{C}, \widetilde{\omega}}:=\left(F^{H_{t}, \check{C}, \widetilde{\omega}}\right)_{t \in \mathbb{T}}$ be as in Lemma 2.6. Then for every capped loop $\gamma=(\widetilde{\gamma}, \check{\gamma})$ whose associated loop $\bar{\gamma}: \mathbb{T} \longrightarrow M$ satisfies $\bar{\gamma}(\mathbb{T}) \subset M-([1+\epsilon / 4,1+\epsilon / 2] \times C)$, we have

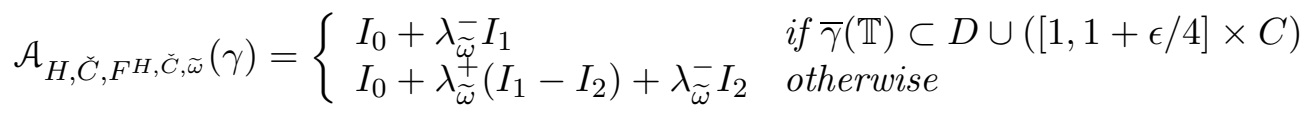

where

$$
\left.I_{0}:=-\int_{S} \widetilde{\gamma}^{*} \widetilde{\omega}, \quad I_{1}:=\int_{0}^{1} H_{t}(\bar{\gamma}(t))\right) d t, \quad I_{2}:=\int_{0}^{1} m_{H_{t}} d t .
$$

Remark 2.9. The $\left(H, \widetilde{\omega}, F^{H_{t}, \breve{C}, \widetilde{\omega}}\right)$-action of a capped loop $\gamma$ satisfying the conditions of Corollary 2.8, only depends on $\omega, H, \check{C}, \lambda_{\widetilde{\omega}}^{ \pm}$and the relative cohomology class $\left[\widetilde{\omega}-\lambda_{\widetilde{\omega}}^{-} \omega\right] \in$ $H^{2}(M, D ; \mathbb{R})$.

The following definition packages together all the necessary action values stated above.

Definition 2.10. Let $\omega_{\check{C}}$ be a $\check{C}$-compatible 2 -form with scaling constants 0 and 1 and which is equal to $\omega$ outside $D \cup([1,1+\epsilon / 2] \times C)$. Let $Q_{\check{C}} \subset H^{2}(M, D ; \mathbb{R}) \times \mathbb{R} \times \mathbb{R}$ be the subset consisting of all triples $\left(q, \lambda^{-}, \lambda^{+}\right)$satisfying $q=\left[\widetilde{\omega}-\lambda^{-} \omega+\lambda^{-} \omega_{\check{C}}\right]$ for some $\check{C}$-compatible 2 -form $\widetilde{\omega} \in \Omega^{2}(M)$ whose scaling constants are $\lambda^{ \pm}$satisfying $\lambda^{-} \leq \lambda^{+}$. For any capped loop $\gamma=(\widetilde{\gamma}, \check{\gamma})$ whose associated loop has image disjoint from $[1-\epsilon / 4,1+\epsilon / 2] \times C$, we define the $(H, \check{C})$-action of $\gamma$ to be the function

$$
\mathcal{A}_{H, \check{C}}(\gamma): Q_{\check{C}} \longrightarrow \mathbb{R}, \quad \mathcal{A}_{H, \check{C}}(\gamma)\left(q, \lambda^{-}, \lambda^{+}\right):=\mathcal{A}_{H, \check{C}, F^{H, \check{C}, \breve{\omega}}}(\gamma)
$$


where $\widetilde{\omega} \in \Omega^{2}(M)$ is a $\check{C}$-compatible 2 -form with scaling constants $\lambda^{ \pm}$satisfying $\left[\widetilde{\omega}-\lambda^{-} \omega+\right.$ $\left.\lambda^{-} \omega_{\check{C}}\right]=q$ and $\lambda^{-} \leq \lambda^{+}$.

The $(H, \check{C})$-action of $\gamma$ is well defined by Remark 2.9 and it does not depend on the choice of $\omega_{\check{C}}$.

Example 2.11. Let $\check{C}, \widetilde{\omega}, \lambda_{\widetilde{\omega}}^{ \pm},\left(\lambda_{H_{t}}\right)_{t \in \mathbb{T}},\left(m_{H_{t}}\right)_{t \in \mathbb{T}}, F^{H, \check{C}, \widetilde{\omega}}:=\left(F^{H_{t}, \check{C}, \widetilde{\omega}}\right)_{t \in \mathbb{T}}$ be as in Lemma 2.6. Suppose that the contact cylinder $\breve{C}$ is the empty set and $\widetilde{\omega}=\omega$. Then for each capped loop $\gamma=(\widetilde{\gamma}, \check{\gamma})$, we get

$$
\mathcal{A}_{H, \emptyset}(\gamma)([\widetilde{\omega}], a, 1)=-\int_{S} \widetilde{\gamma}^{*} \omega+\int_{0}^{1} H_{t}(\bar{\gamma}(t)) d t
$$

for all $a \in \mathbb{R}$ which is the usual action functional defined in, say, [Flo88] (with different sign conventions) where $S$ is the domain of $\widetilde{\gamma}$ and $\bar{\gamma}$ is the associated loop of $\gamma$.

2.2. Floer Trajectories. In this section we will give a definition of a Floer trajectory converging to a collection of capped 1-periodic orbits and state some results concerning spaces of Floer trajectories. Throughout this subsection, we will fix a (possibly empty) contact cylinder $\check{C}=[1-\epsilon, 1+\epsilon] \times C \subset M$.

Definition 2.12. Let $\mathcal{H}^{\Sigma}(\check{C})$ be the space of smooth families of Hamiltonians $H^{\bullet}=\left(H^{\sigma}\right)_{\sigma \in \Sigma}$ parameterized by a manifold $\Sigma$ which are weakly $\check{C}$-compatible and equipped with the $C_{\text {loc }}^{\infty}$ topology. Let $\overline{\mathcal{H}}^{\Sigma}(\check{C}) \subset \mathcal{H}^{\Sigma}(\check{C})$ be the subspace consisting of those $H$ which are $\check{C}$ compatible. Let $\mathcal{J}^{\Sigma}(\check{C})$ be the space of smooth families $J^{\bullet}=\left(J^{\sigma}\right)_{\sigma \in \Sigma}$ of almost complex structures on $M$ which are $\check{C}$-compatible equipped with the $C_{\mathrm{loc}}^{\infty}$ topology.

Definition 2.13. (See Figure 4.) A Riemann surface with $n_{-}$negative cylindrical ends and $n_{+}$positive cylindrical ends is a Riemann surface $\Sigma$ together with a collection of proper embeddings

where

$$
\left(\iota_{j}: \mathbb{I}_{ \pm} \times \mathbb{T} \longrightarrow \Sigma\right)_{j \in I_{-} \sqcup I_{+}}
$$

- $I_{-}, I_{+}$are finite indexing sets and the images of $\iota_{j}$ are all disjoint from each other,

- $\Sigma$ is biholomorphic to $\check{\Sigma}-\cup_{j \in I_{-} \sqcup I_{+}}\left\{p_{j}\right\}$ where $\Sigma$ is a closed Riemann surface and $\left(p_{j}\right)_{j \in I_{-} \sqcup I_{+}}$are distinct points in $\check{\Sigma}$ and

- for each $j \in I_{ \pm}$there is a holomorphic chart $U_{j}$ in $\check{\Sigma}$ centered at $p_{j}$ so that $\operatorname{Im}\left(\iota_{j}\right) \subset U_{j}$ and

$$
\iota_{j}(s, t)=e^{\mp 2 \pi(s+i t)}
$$

with respect to this chart.

The map $\iota_{j}$ is called the negative (resp. positive) cylindrical end associated to $j \in I_{-}$ (resp. $\left.j \in I_{+}\right)$. The coordinate $(s+i t) \circ \iota_{j}^{-1}: \operatorname{Im}\left(\iota_{j}\right) \longrightarrow \mathbb{C}$ on the negative (resp. positive) cylindrical end $\iota_{j} \in I_{ \pm}$is called the cylindrical coordinate associated to $j \in I_{-} \sqcup I_{+}$.

Definition 2.14. Let $\Sigma$ be a Riemann surface with $n_{-}$negative cylindrical ends and $n_{+}$ positive cylindrical ends labeled by finite sets $I_{-}$and $I_{+}$respectively. A 1-form $\beta \in \Omega^{1}(\Sigma)$ is $\Sigma$-compatible if $\iota_{j}^{*} \beta=\kappa_{j} d t$ for all $j \in I_{-} \sqcup I_{+}$where $\left(\kappa_{i}\right)_{i \in I_{-}} \sqcup I_{+}$are positive constants. Here $\kappa_{j}$ is called the weight of $\beta$ at the cylindrical end corresponding to $j$. A smooth family of tensor fields $\alpha:=\left(\alpha_{z}\right)_{z \in \Sigma}$ on $M$ (e.g. functions, differential forms, almost complex structures) is $\Sigma$ compatible if there is a compact subset $K_{\alpha} \subset \Sigma$ and a smooth family of tensors $\alpha^{j}:=\left(\alpha_{t}^{j}\right)_{t \in \mathbb{T}}$ 


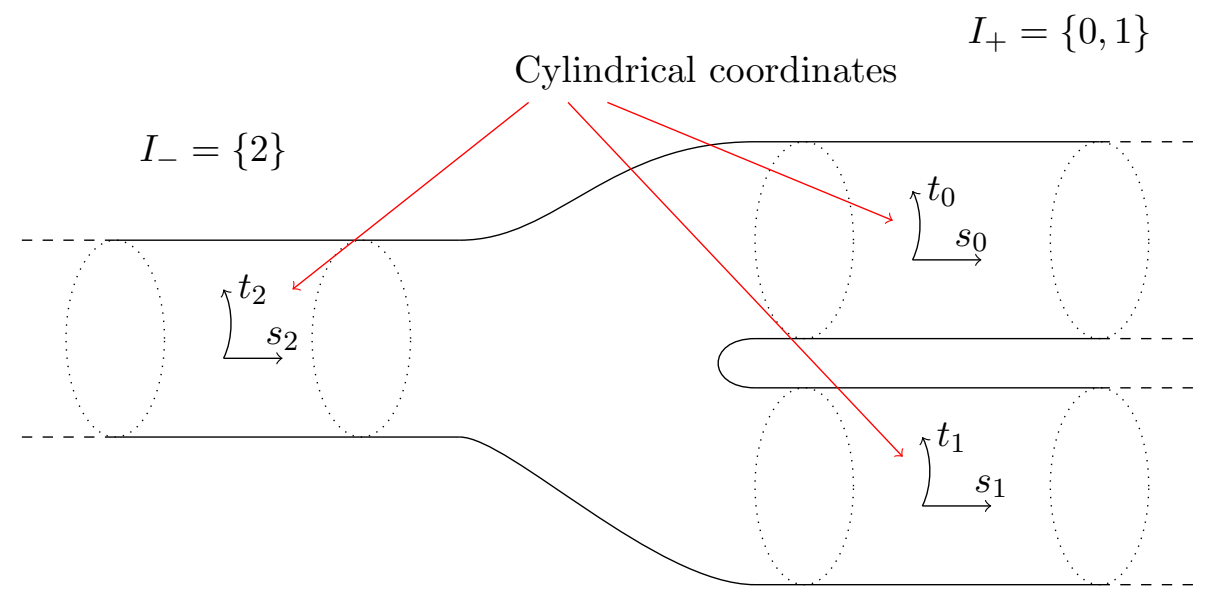

FIGURE 4. Riemann surface with one negative cylindrical end and two positive cylindrical ends

on $M$ for each $j \in I_{-} \sqcup I_{+}$so that $\alpha_{\iota_{j}(s, t)}=\alpha_{t}^{j}$ for all $(s, t) \in \mathbb{I}_{ \pm} \times \mathbb{T}$ satisfying $\iota_{j}(s, t) \notin K_{\alpha}$ and all $j \in I_{-} \sqcup I_{+}$. Here $\alpha^{j}$ is the limit of $\alpha$ corresponding to $j \in I_{-} \sqcup I_{+}$and $\alpha^{\#}:=\left(\alpha^{j}\right)_{j \in I_{-} \sqcup I_{+}}$ are the limits of $\alpha$. Let $\widetilde{\omega} \in \Omega^{2}(M)$ be $\check{C}$-compatible. A smooth family of autonomous Hamiltonians $H:=\left(H_{z}\right)_{z \in \Sigma}$ on $M$ is $(\Sigma, \check{C})$-admissible if

(1) $H \in \mathcal{H}^{\Sigma}(\check{C})$ and $H$ is $\Sigma$-compatible,

(2) $d\left(h^{x} \beta\right), d(\lambda \beta), d(m \beta), d\left(\left(h^{x}-\lambda\right) \beta\right) \leq 0$ for all $x \in M$ where $h^{x}, \lambda$ and $m$ are maps from $\Sigma$ to $\mathbb{R}$ defined by:

$$
h^{x}(\sigma):=H_{\sigma}(x), \quad \lambda(\sigma):=\lambda_{H_{\sigma}}, \quad m(\sigma):=m_{H_{\sigma}} \quad \forall \sigma \in \Sigma
$$

where $\left(\lambda_{H_{\sigma}}\right)_{\sigma \in \Sigma}$ and $\left(m_{H_{\sigma}}\right)_{\sigma \in \Sigma}$ are the slopes and heights of $H=\left(H_{\sigma}\right)_{\sigma \in \Sigma}$ respectively.

Let $H^{\#}:=\left(H^{j}\right)_{j \in I_{-} \sqcup I_{+}}$be a collection of Hamiltonians. We define $\mathcal{H}^{\Sigma}\left(H^{\#}, \check{C}\right)$ to be the set of $(\Sigma, \check{C})$-admissible smooth families of Hamiltonians whose limits are $H^{\#}$. We also define $\overline{\mathcal{H}}^{\Sigma}\left(H^{\#}, \check{C}\right):=\overline{\mathcal{H}}^{\Sigma}(\check{C}) \cap \mathcal{H}^{\Sigma}\left(H^{\#}, \check{C}\right)$.

Let $J^{j}:=\left(J_{t}^{j}\right)_{t \in \mathbb{T}}$ be a smooth family of almost complex structures in $J^{\mathbb{T}}(\check{C})$ for each $j \in I_{-} \sqcup I_{+}$and let $J^{\#}:=\left(J^{j}\right)_{j \in I_{-} \sqcup I_{+}}$. Define $\mathcal{J}^{\Sigma}\left(J^{\#}, \check{C}\right) \subset \mathcal{g}^{\Sigma}(\check{C})$ to be the subspace of $\Sigma$-compatible families $J=\left(J_{z}\right)_{z \in \Sigma}$ of almost complex structures whose limits are $J^{\#}$.

Now let $H \in \mathcal{H}^{\Sigma}\left(H^{\#}, \check{C}\right)$ and $J \in \mathcal{J}^{\Sigma}\left(J^{\#}, \check{C}\right)$ for some $H^{\#}, J^{\#}$ as above and let $\mathbf{j}$ be the natural complex structure on $\Sigma$. We say that $u: \Sigma \longrightarrow M$ satisfies the Floer equation with respect to $(H, J)$ if

$$
\left(d u+X_{H_{\sigma}} \otimes \beta\right)+J_{\sigma} \circ\left(d u+X_{H_{\sigma}} \otimes \beta\right) \circ \mathbf{j}=0
$$

at each point $\sigma \in \Sigma$. A continuous map $u: \Sigma \longrightarrow M$ converges to capped 1-periodic orbits $\gamma^{\#}:=\left(\left(\widetilde{\gamma}^{j}, \check{\gamma}^{j}\right)\right)_{j \in I_{-} \sqcup I_{+}}$of $\left(\kappa_{j} H^{j}\right)_{j \in I_{-} \sqcup I_{+}}$respectively if

- $\lim _{s \rightarrow \pm \infty} u\left(\iota_{j}(s, t)\right)=\bar{\gamma}^{j}(t)$ for all $j \in I_{ \pm}$where $\bar{\gamma}^{j}$ is the associated loop of $\left(\widetilde{\gamma}^{j}, \check{\gamma}^{j}\right)$ and

- the surface obtained by gluing the ends of $u$ with the surfaces $\left(\widetilde{\gamma}^{j}\right)_{j \in I_{-} \sqcup I_{+}}$is nullhomologous in $H_{2}(M ; \mathbb{Z})$. 
We let $\mathcal{M}\left(H, J, \gamma^{\#}\right)$ be the space of maps $u: \Sigma \longrightarrow M$ satisfying the Floer equation with respect to $(H, J)$ and converging to $\gamma^{\#}$ equipped with the $C_{\text {loc }}^{\infty}$ topology.

Remark 2.15. The space $\mathcal{M}\left(H, J, \gamma^{\#}\right)$ also depends on $\beta$ but we omit this from the notation as it is either clear which $\beta$ we are using, or if $\beta$ isn't mentioned then we will assume some $\beta$ has been chosen.

The motivation for part (2) of the definition of a $(\Sigma, \check{C})$-admissible Hamiltonian above is that it ensures that, roughly, that the Floer complex (defined in Section 2.5) is filtered by the $(H, \check{C})$-action from Definition 2.10. See also Lemma 10.3 combined with Equation (2.5).

Definition 2.16. Each capped 1-periodic orbit $\gamma$ can be assigned an index $C Z(\gamma)$ called the Conley-Zehnder index. Such an index is defined in the following way: To any path $A:=\left(A_{t}\right)_{t \in[a, b]}$ of symplectic matrices, we can assign an index $C Z(A)$ called its ConleyZehnder index ([CZ84], [RS93] and [Gut14]). We will not give a definition here, but state some important properties (See [Gut14, Proposition 6], [Gut14, Lemma 26] and [McL16, Corollary 4.9]):

(CZ1) $C Z\left(\left(e^{i t}\right)_{t \in[0,2 \pi]}\right)=2$.

(CZ2) $C Z(A \oplus B)=C Z(A)+C Z(B)$ where $A=\left(A_{t}\right)_{t \in[a, b]}, B=\left(B_{t}\right)_{t \in[a, b]}$ are paths of symplectic matrices and $A \oplus B:=\left(A_{t} \oplus B_{t}\right)_{t \in[a, b]}$.

(CZ3) The Conley-Zehnder index of the catenation of two paths is the sum of their ConleyZehnder indices.

(CZ4) If $A$ and $B$ are two paths of symplectic matrices which are homotopic relative to their endpoints then they have the same Conley-Zehnder index. Also such an index only depends on the path up to orientation preserving reparameterization.

(CZ5) Let

$$
A_{t}=\left(\begin{array}{cc}
\mathrm{id} & -t B \\
0 & \mathrm{id}
\end{array}\right) \in G L(2 n ; \mathbb{R}), \quad \forall t \in[0,1]
$$

be a family of $2 n \times 2 n$ matrices where id is the identity $n \times n$ matrix and $B$ is a symmetric $n \times n$ matrix. Let $A:=\left(A_{t}\right)_{t \in[0,1]}$ be a path of symplectic matrices with respect to the linear symplectic form $\Omega=\sum_{i=1}^{n} x_{i}^{*} \wedge y_{i}^{*}$ where $x_{1}^{*}, \cdots, x_{n}^{*}, y_{1}^{*}, \cdots, y_{n}^{*}$ are the dual basis vectors of the standard basis $x_{1}, \cdots, x_{n}, y_{1}, \cdots, y_{n}$ of $\mathbb{R}^{2 n}$. Then $C Z(A)=\frac{1}{2} \operatorname{Sign}(B)$.

(CZ6) Let $\left(A_{t}\right)_{t \in[0,1]}$ be a path of symplectic matrices so that $\operatorname{dim}\left(\operatorname{ker}\left(A_{t}-\mathrm{id}\right)\right)$ is independent of $t$. Then $C Z\left(\left(A_{t}\right)_{t \in[0,1]}\right)=0$.

(CZ7) Let $S p(2 n)$ be the space of symplectic $2 n \times 2 n$ matrices and let $A \in S p(2 n)$. Then there is a neighborhood $N_{A}$ of $A$ so that any path $\left(A_{t}\right)_{t \in[0,1]}$ in $N_{A}$ with $A_{0}=A$ satisfies $C Z\left(\left(A_{t}\right)_{t \in[0,1]}\right) \in\left[-\frac{k}{2}, \frac{k}{2}\right]$ where $k=\operatorname{dim} \operatorname{ker}(A-\mathrm{id})$.

The Conley-Zehnder index of a capped 1-periodic orbit $\gamma=(\widetilde{\gamma}, \check{\gamma})$ of $H$ is given by the Conley-Zehnder index of

$$
\left.\left.\tau\right|_{\check{\gamma}(t)} \circ D \phi_{t}^{H}\right|_{\check{\gamma}(0)} \circ\left(\left.\tau\right|_{\check{\gamma}(0)}\right)^{-1}, \quad t \in[0,1]
$$

where $\tau: \widetilde{\gamma}^{*} T M \longrightarrow S \times \mathbb{C}^{n}$ is a symplectic trivialization over the domain $S$ of $\widetilde{\gamma}$ and $\left.\tau\right|_{\sigma}:\left.\widetilde{\gamma}^{*}(T M)\right|_{\sigma} \longrightarrow \mathbb{C}^{n}$ is its restriction to the fiber $\sigma \in \Sigma$. We define the index of $\gamma$ to be $|\gamma|:=n-C Z(\gamma)$. If $\left(\gamma_{j}\right)_{j \in I}$ is a finite collection of capped 1-periodic orbits then we define $\left|\left(\gamma_{j}\right)_{j \in I}\right|:=\sum_{j \in I}\left|\gamma_{j}\right|$.

Remark 2.17. Such an index does not depend on the choice of trivialization $\tau$ by (CZ4) combined with the fact that $\pi_{1}(S p(2 n))$ is abelian. Also since $c_{1}(M)=0$, the index only 
depends on the associated loop $\bar{\gamma}$. Therefore, we will define $|\bar{\gamma}|:=|\gamma|$ for any associated loop $\bar{\gamma}$ of a capped 1-periodic orbit $\gamma$.

Definition 2.18. Let $T$ be a topological space. A subset $S \subset T$ is ubiquitous if it contains a countable intersection of dense open sets.

We have the following important proposition:

Proposition 2.19. Let $\Sigma, H, J^{\#},\left(\kappa_{j}\right)_{j \in I}$ be as in Definition 2.14. Then there is a ubiquitous subset $\mathrm{J}^{\Sigma, \mathrm{reg}}\left(H, J^{\#}, \check{C}\right) \subset \mathcal{J}^{\Sigma}\left(J^{\#}, \check{C}\right)$ so that a certain family of linearized operators is surjective and so certain other transversality conditions hold (see Definition 9.10).

Also, suppose that we have a collection $\gamma^{\#}:=\left(\gamma^{j}\right)_{j \in I_{-} \sqcup I_{+}}$of non-degenerate capped 1periodic orbits of $\left(\kappa_{j} H^{j}\right)_{j \in I_{-} \sqcup I_{+}}$whose associated 1-periodic orbits are disjoint from $V$ and so that if $\Sigma \neq \mathbb{R} \times \mathbb{T}$ then at least two such 1-periodic orbits of $\gamma^{\#}$ have distinct images. Then for each $J \in \mathcal{J}^{\Sigma, \operatorname{reg}}\left(H, J^{\#}, \check{C}\right), \mathcal{M}\left(H, J, \gamma^{\#}\right)$ is an oriented $k$-dimensional manifold where

$$
k:=\left|\left(\gamma^{j}\right)_{j \in I_{-}}\right|-\left|\left(\gamma^{j}\right)_{j \in I_{+}}\right|+n\left(|I|_{+}-|I|_{-}+\chi(\Sigma)\right)
$$

where $\chi(\Sigma)$ is the Euler characteristic of $\Sigma$. Also if the dimension of $\mathcal{M}\left(H, J, \gamma^{\#}\right)$ is $\leq 1$ then we can ensure that the image of each $u \in \mathcal{M}\left(H, J, \gamma^{\#}\right)$ is disjoint from $V$.

The proof is extremely standard using ideas from [MS98, Chapters 3 and 6] and from [Sch95]. However for the sake of completeness, we will prove this proposition in Appendix B. Note that $\mathcal{M}\left(H, J, \gamma^{\#}\right)$ may not be a manifold at all if any of the capped 1-periodic orbits $\gamma^{j}$ are degenerate even if $J \in \mathcal{J}^{\Sigma, \mathrm{reg}}\left(H, J^{\#}, \check{C}^{\#}\right)$ by our current definition. We also have a 1-parameter version of this proposition stated below.

Definition 2.20. Let $\Sigma_{\bullet}:=\left(\Sigma_{s}\right)_{s \in[0,1]}$ be a smooth family of Riemann surfaces with $n_{-}$ negative cylindrical ends and $n_{+}$positive cylindrical ends labeled by finite sets $I_{-}$and $I_{+}$ respectively. Let $\left(\kappa_{j}\right)_{j \in I_{-} \sqcup I_{+}}$be positive numbers and let $\beta_{s}$ be a $\Sigma_{s}$-compatible 1-form so that $\kappa_{j}$ is the weight of $\beta_{s}$ at the cylindrical end corresponding to $j \in I_{-} \sqcup I_{+}$for each $j \in I_{-} \sqcup I_{+}, s \in[0,1]$ and so that $\left(\beta_{s}\right)_{s \in[0,1]}$ is a smooth family of 1-forms.

Let $H^{\#}:=\left(H_{i}\right)_{i \in I_{-} \sqcup I_{+}}$be elements of $\mathcal{H}^{\mathbb{T}}(\check{C})$. We define $\mathcal{H}^{\Sigma} \bullet\left(H^{\#}, \check{C}\right)$ (resp. $\left.\overline{\mathcal{H}}^{\Sigma \bullet}\left(H^{\#}, \check{C}\right)\right)$ to be the space of smooth families of Hamiltonians $H:=\left(H_{s, \sigma}\right)_{s \in[0,1], \sigma \in \Sigma_{s}}$ so that the subfamily $H_{s, \bullet}:=\left(H_{s, \sigma}\right)_{\sigma \in \Sigma_{s}}$ is an element of $\mathcal{H}^{\Sigma_{s}}\left(H^{\#}, \check{C}\right)\left(\operatorname{resp} \overline{\mathcal{H}}^{\Sigma_{s}}\left(H^{\#}, \check{C}\right)\right)$ for each $s \in[0,1]$. Similarly let $\mathcal{J}^{\Sigma} \bullet\left(J^{\#}, \check{C}\right)$ be the space of smooth families of almost complex structures $J:=\left(J_{s, \sigma}\right)_{s \in[0,1], \sigma \in \Sigma_{s}}$ so that the subfamily $J_{s, \bullet}:=\left(J_{s, \sigma}\right)_{\sigma \in \Sigma_{s}}$ is an element of $\mathcal{g}^{\Sigma_{s}}\left(J^{\#}, \check{C}\right)$ for each $s \in[0,1]$. For each $H \in \mathcal{H}^{\Sigma} \bullet\left(H^{\#}, \check{C}\right)$ and each $Y_{j} \in \mathcal{J}^{\Sigma_{j}, \operatorname{reg}}\left(H_{j, \bullet}, J^{\#}, \check{C}\right)$ for $j=0,1$, define $g^{\Sigma} \bullet\left(\left(Y_{0}, Y_{1}\right), J^{\#}, \check{C}^{\#}\right)$ to be the subspace of $g^{\Sigma} \bullet\left(J^{\#}, \check{C}\right)$ consisting of those $J$ as above satisfying $J_{0, \bullet}=Y_{0}$ and $J_{1, \bullet}=Y_{1}$. For each $J \in \mathcal{J}^{\Sigma} \bullet\left(\left(Y_{0}, Y_{1}\right), J^{\#}, \check{C}\right)$ as above and each tuple of capped 1-periodic orbits $\gamma^{\#}:=\left(\gamma^{j}\right)_{j \in I_{-} \sqcup I_{+}}$of $\left(\kappa_{j} H^{j}\right)_{j \in I_{-} \sqcup I_{+}}$, define $\mathcal{M}\left(H, J, \gamma^{\#}\right):=\sqcup_{s \in[0,1]} \mathcal{M}\left(H_{s, \bullet}, J_{s, \bullet}, \gamma^{\#}\right)$ with the induced $C_{\text {loc }}^{\infty}$ topology.

We then have the following parameterized version of Proposition 2.19 above. We will not prove this since the ideas used to prove it are exactly the same as those from Proposition 2.19 .

Proposition 2.21. Let $\Sigma_{\bullet}, H^{\#}, J^{\#},\left(\kappa_{j}\right)_{j \in I}$ be as in Definition 2.20. Suppose $H \in$ $\mathcal{H}^{\Sigma} \bullet\left(H^{\#}, \check{C}\right), Y_{j} \in \mathcal{J}^{\Sigma_{j}, \mathrm{reg}}\left(H_{j, \bullet}, J^{\#}, \check{C}\right)$ for $j=0,1$ where $H_{s, \bullet}$ is described in the previous definition. Then there is a ubiquitous subset

$$
g^{\Sigma \bullet}, \operatorname{reg}\left(H,\left(Y_{0}, Y_{1}\right), J^{\#}, \check{C}\right) \subset g^{\Sigma} \bullet\left(\left(Y_{0}, Y_{1}\right), J^{\#}, \check{C}\right)
$$


so that a certain family of linearized operators is surjective and so certain other transversality conditions hold.

Also suppose $\gamma^{\#}:=\left(\gamma^{j}\right)_{j \in I_{-} \sqcup I_{+}}$are capped 1-periodic orbits of $\left(\kappa_{j} H^{j}\right)_{j \in I_{-} \sqcup I_{+}}$so that $\gamma^{j}$ is non-degenerate for each $j \in I_{-} \sqcup I_{+}$, whose associated 1-periodic orbits are disjoint from $V$ and so that if $\Sigma \neq \mathbb{R} \times \mathbb{T}$ then at least two associated 1-periodic orbits of $\gamma^{\#}$ have distinct images. Then for each $J \in \mathcal{J}^{\Sigma_{\bullet}, r^{r e g}}\left(H,\left(Y_{0}, Y_{1}\right), J^{\#}, \check{C}\right), \mathcal{M}\left(H, J, \gamma^{\#}\right)$ is an oriented $k+1$ dimensional manifold with boundary equal to $\sqcup_{j=0}^{1} \mathcal{M}\left(H_{j, \bullet}, Y_{j}, \gamma^{\#}\right)$ where $k$ is defined as in equation (2.9). Also if the dimension of $\mathcal{M}\left(H, J, \gamma^{\#}\right)$ is $\leq 1$ then we can ensure that the image of each $u \in \mathcal{M}\left(H, J, \gamma^{\#}\right)$ is disjoint from $V$.

Example 2.22. Let $\mathbb{R} \times \mathbb{T}=\mathbb{C} / \mathbb{Z}$ be a Riemann surface with a positive cylindrical end indexed by $I_{+}:=\{+\}$and a negative cylindrical end indexed by $I_{-}:=\{-\}$given by the natural inclusion maps into $\mathbb{R} \times \mathbb{T}$. Also let $\beta:=d t$ and $\kappa_{ \pm}:=1$ be the corresponding weights of $\beta$. Here $\mathbb{R} \times \mathbb{T}$ along with $\beta$ and $\kappa_{ \pm}$is called a Riemann cylinder. Let $H$ be $(\mathbb{R} \times \mathbb{T}, \check{C})$ compatible with associated limits $H^{ \pm}$. Also let $J^{ \pm} \in \mathcal{J}^{\mathbb{T}}(\check{C})$ and let $J \in \mathcal{J}^{\mathbb{R} \times \mathbb{T}}\left(\left(J^{+}, J^{-}\right), \check{C}\right)$. Then a map $u: \mathbb{R} \times \mathbb{T} \longrightarrow M$ satisfying the $(H, J)$-Floer equation and converging to capped 1-periodic orbits $\gamma_{-}, \gamma_{+}$of $H^{-}, H^{+}$respectively is called an $(H, J)$-Floer cylinder connecting $\gamma_{-}$and $\gamma_{+}$.

Definition 2.23. Let $\mathbb{R} \times \mathbb{T}$ be a Riemann cylinder. Let

$$
\begin{gathered}
\iota_{\mathrm{Ham}}: \mathcal{H}^{\mathbb{T}}(\check{C}) \longleftrightarrow \bigcup_{H^{\#}=\left(H^{-}, H^{+}\right) \in\left(\mathcal{H}^{\mathbb{T}}(\check{C})\right)^{2}} \mathcal{H}^{\mathbb{R} \times \mathbb{T}}\left(H^{\#}, \check{C}\right) \\
\iota_{\mathrm{cpx}}: \mathcal{J}^{\mathbb{T}}(\check{C}) \longleftrightarrow \bigcup_{J^{\#}=\left(J^{-}, J^{+}\right) \in\left(\mathcal{J}^{\mathbb{T}}(\check{C})\right)^{2}} \mathcal{J}^{\mathbb{R} \times \mathbb{T}}\left(J^{\#}, \check{C}\right)
\end{gathered}
$$

be the natural embeddings sending $\left(H_{t}\right)_{t \in \mathbb{T}}$ to $\left(H_{t}\right)_{(s, t) \in \mathbb{R} \times \mathbb{T}}$ and $\left(J_{t}\right)_{t \in \mathbb{T}}$ to $\left(J_{t}\right)_{(s, t) \in \mathbb{R} \times \mathbb{T}}$ respectively.

Also for each $(H, J) \in \mathcal{H}^{\mathbb{T}}(\check{C}) \times \mathcal{J}^{\mathbb{T}}(\check{C})$ and for each pair $\gamma^{\#}:=\left(\gamma^{-}, \gamma^{+}\right)$of capped 1-periodic orbits of $H$, we define

$$
\mathcal{M}\left(H, J, \gamma^{\#}\right):=\mathcal{M}\left(\iota_{\text {Ham }}(H), \iota_{\text {cpx }}(J), \gamma^{\#}\right), \quad \overline{\mathcal{M}}\left(H, J, \gamma^{\#}\right):=\mathcal{M}\left(H, J, \gamma^{\#}\right) / \mathbb{R}
$$

where the natural $\mathbb{R}$-action is given by translation in the $s$ coordinate. Also for each $H \in$ $\mathcal{H}^{\mathbb{T}}(\check{C})$, define

$$
\mathcal{J}^{\mathbb{T}, \text { reg }}(H, \check{C}):=\mathcal{J}^{\mathbb{T}}(\check{C}) \cap \iota_{\text {cpx }}^{-1}\left(\cup_{J \in \mathcal{J}^{\mathbb{T}}(\check{C})} \mathcal{J}^{\mathbb{R} \times \mathbb{T}, \mathrm{reg}}\left(\iota_{\mathrm{Ham}}(H),(J, J), \check{C}\right)\right) .
$$

We also have the following proposition (see [Ush11, Proposition 3.4]):

Proposition 2.24. For each $H \in \mathcal{H}^{\mathbb{T}}(\check{C})$, $\mathrm{J}^{\mathbb{T}, \mathrm{reg}}(H, \check{C})$ is a ubiquitous subset of $\mathcal{J}^{\mathbb{T}}(\check{C})$. Also for each pair of non-degenerate capped 1-periodic orbits $\gamma_{-}, \gamma_{+}$of $H$, we have that $\overline{\mathcal{M}}\left(H, J, \gamma^{\#}\right)$ is a manifold of dimension $\left|\gamma_{-}\right|-\left|\gamma_{+}\right|-1$. If the dimension of $\overline{\mathcal{M}}\left(H, J, \gamma^{\#}\right)$ is $\leq 1$ then each element of $\overline{\mathcal{M}}\left(H, J, \gamma^{\#}\right)$ has image disjoint from $V$.

Example 2.25. Let $\Sigma:=\mathbb{P}^{1}-\{0,1, \infty\}$ and let $\varpi: \Sigma \longrightarrow \mathbb{C}^{*}$ be a proper holomorphic map of degree 2 with exactly one branch point at $1 \in \mathbb{C}^{*}$ and so that $\varpi^{-1}\left(\left\{z \in \mathbb{C}^{*}:|z|<1\right\}\right)$ is connected (See Figure 5). 


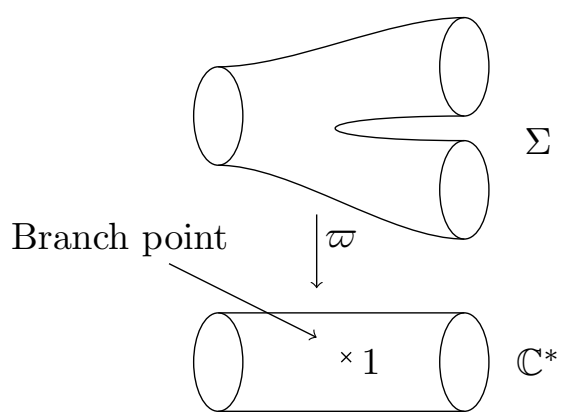

FiguRE 5. Branched cover of pair of pants over a cylinder.

Let $I_{-}:=\{2\}$ and $I_{+}:=\{0,1\}$. Choose cylindrical ends

$$
\iota_{j}: \mathbb{I}_{ \pm} \times \mathbb{T} \longrightarrow \Sigma, \quad j \in I_{ \pm}
$$

for $\Sigma$ so that $\varpi\left(\iota_{j}(s, t)\right)=e^{2 \pi(s+1+i t)}$ for $j \in I_{+}$and $\varpi\left(\iota_{2}(s, t)\right)=e^{2 \pi(s-1+2 i t)}$. Then $\beta:=\varpi^{*} d(\arg (z) / 2 \pi)$ is a $\Sigma$-compatible 1 -form so that the weight $\kappa_{2}$ of $\beta$ is 2 at the negative end and the weights $\kappa_{0}, \kappa_{1}$ are 1 at each positive end. We call $\Sigma$ with its negative and two positive cylindrical ends above together with the 1 -form $\beta$ the pair of pants.

Let $H^{\#}=\left(H^{j}\right)_{j \in\{0,1,2\}}$ where $H^{j} \in \mathcal{H}^{\mathbb{T}}(\check{C})$ for $j=0,1,2$ and let $H \in \mathcal{H}^{\mathbb{T}}\left(H^{\#}, \check{C}\right)$. Similarly let $J^{\#}=\left(J^{i}\right)_{i \in\{0,1,2\}}$ where $J^{j} \in \mathcal{J}^{\mathbb{T}}(\check{C})$ where $j=0,1,2$, define and let $J \in$ $\mathcal{J}\left(J^{\#}, \check{C}\right)$. Let $\gamma_{j}$ be a capped 1-periodic orbit of $\kappa_{j} H_{j}$ for $j=0,1,2$ and define $\gamma^{\#}:=$ $\left(\gamma_{j}\right)_{j \in\{0,1,2\}}$. An $(H, J)$-pair of pants connecting $\gamma^{\#}$ is an $(H, J)$-Floer trajectory connecting the capped 1-periodic orbits $\gamma^{\#}$.

2.3. Directed and Inverse Systems. In this subsection we will give some definitions concerning inverse/direct limits, which will be used later to define symplectic cohomology. A small part of this section will also be used in the next section to define Novikov rings. Many of the main ideas come from [MS82] and [Mar00]. We will also introduce a category of "double systems" which, alternatively, is a category of appropriate "ind-pro" objects (I.e. direct limits of inverse limits of modules). Such categories have appeared in [Bei87, Section A.3] and [Kat00] for example.

Definition 2.26. If $\left(I, \leq_{I}\right),\left(K, \leq_{K}\right)$ are sets together relations $\leq_{I}$ and $\leq_{K}$ then we define $\left(I \times K, \leq_{I \times K}\right)$ to be the product $I \times K$ together with the relation $\leq_{I \times K}$ satisfying $(i, k) \leq_{I \times K}$ $\left(i^{\prime}, k^{\prime}\right)$ if $i \leq_{I} i^{\prime}$ and $k \leq_{K} k^{\prime}$. Also we define $\left(I^{\mathrm{op}}, \leq^{\mathrm{op}}\right)$ to be the set $I^{\mathrm{op}}:=I$ together with the relation $\leq_{I}^{\text {op }}$ satisfying $i \leq_{I}^{\text {op }} i^{\prime}$ if $i^{\prime} \leq_{I} i$ for all $i, i^{\prime} \in I^{\text {op }}$.

A directed set $\left(I, \leq_{I}\right)$ is a set $I$ together with a reflexive transitive binary relation $\leq_{I}$ so that for all $i_{0}, i_{1} \in I$, there exists $i_{2} \in I$ satisfying $i_{0}, i_{1} \leq_{I} i_{2}$. An inverse directed set $\left(K, \leq_{K}\right)$ is a set $K$ together with a relation $\leq_{K}$ so that $\left(K^{\mathrm{op}}, \leq_{K}^{\mathrm{op}}\right)$ is a directed set.

If $\left(I, \leq_{I}\right)$ is a directed set, then a subset $\check{I} \subset I$ is cofinal if for each $a \in I$, there exists $\check{a} \in \check{I}$ satisfying $a \leq_{I} \check{a}$. A sequence $\left(a_{k}\right)_{k \in \mathbb{N}}$ of elements in $I$ is cofinal if the set $\left\{a_{k}: k \in \mathbb{N}\right\} \subset I$ is cofinal. A directed subset of $I$ is a subset $\check{I} \subset I$ where the induced ordering makes $\check{I}$ into a directed set. A subset $\check{K} \subset K$ is cofinal inside an inverse directed set $\left(K, \leq_{K}\right)$ if $\check{K}^{\text {op }} \subset K^{\text {op }}$ is cofinal in $\left(K^{\mathrm{op}}, \leq_{K}^{\mathrm{op}}\right)$. A double set is a pair $\left(I \times K, \leq_{I \times K}\right)$ where $\left(I \leq_{I}\right)$ is a directed set and $\left(K, \leq_{K}\right)$ is an inverse directed set.

Remark 2.27. We can think of a set with a reflexive transitive binary relation $(I, \leq)$ as a category with objects $I$ and with a unique morphism denoted by $i \rightarrow j$ for each $i \leq j$ and no 
other morphisms. Since there is at most one morphism between any two objects, a functor $F:\left(I_{0}, \leq\right) \longrightarrow\left(I_{1}, \leq\right)$ is characterized by the corresponding map $F: I_{0} \longrightarrow I_{1}$ on objects. From now on, we will not distinguish between such functors and maps.

For ease of notation, we will sometimes just write $I$ for a set with relation $(I, \leq)$ if it is clear what the relation is. In particular, we will write $I \times K$ instead of $\left(I \times K, \leq_{I \times K}\right)$.

Definition 2.28. Let $R$ be a commutative ring and let $R$-mod be the category of $\mathbb{Z}$-graded $R$-modules. We write $O b(R$-mod $)$ and $M o r(R$-mod $)$ to be the class of objects and morphisms of $R$-mod.

A directed system is a functor $D: I \longrightarrow R$-mod where $\left(I, \leq_{I}\right)$ is a directed set. An inverse system is a functor $V: K \longrightarrow R$-mod where $\left(K, \leq_{K}\right)$ is an inverse directed system. A double system of graded $R$-modules (or double system) is a functor $W: I \times K \longrightarrow R$-mod where $\left(I \times K, \leq_{I \times K}\right)$ is a double set. The morphisms $W\left((i, k) \rightarrow\left(i^{\prime}, k^{\prime}\right)\right) \in \operatorname{Mor}(R$-mod $)$, $(i, k) \leq_{I \times K}\left(i^{\prime}, k^{\prime}\right)$ are called double system morphisms of $W$.

Remark 2.29. We will sometimes just write down a double system $W: I \times K \longrightarrow R$-mod as a collection $(W(i, k))_{(i, k) \in I \times K}$ of $R$-modules if it is clear what the morphisms $W\left((i, k) \rightarrow\left(i^{\prime}, k^{\prime}\right)\right)$ are. We will do the same for directed and inverse systems.

Remark 2.30. A directed system $D: I \longrightarrow R$-mod is equivalent to a double system $D$ : $I=I \times\{\star\} \longrightarrow R$-mod where $\{\star\}$ is the single element (inverse) directed set. Similarly an inverse system is a double system $V: K=\{\star\} \times K \longrightarrow R$-mod. We will call such double systems the double systems associated to $D$ (resp. $V$ ).

Example 2.31. The trivial double system is the double system given by the functor $\iota_{R}$ : $\{\star\} \times\{\star\} \longrightarrow R$-mod sending $(\star, \star)$ to $R$ where $\{\star\}$ is the directed/inverse system consisting of one point. Trivial directed/inverse systems are defined in a similar way.

Definition 2.32. For any $\mathbb{Z}$ graded $R$-module $N$, let $(N)_{p}$ be the degree $p$ part of $N$ for each $p \in \mathbb{Z}$. If $W: I \times K \longrightarrow R$-mod is a double system then we define the double system shifted by $m \in \mathbb{Z}$ to be the unique double system $W[m]: I \times K \longrightarrow R$-mod satisfying $(W[m](i, k))_{p}=(W(i, k))_{m+p}$ for all $p \in \mathbb{Z}$ with the natural induced morphisms.

Definition 2.33. The direct limit of a directed system $D: I \longrightarrow R$-mod is defined to be the graded $R$-module

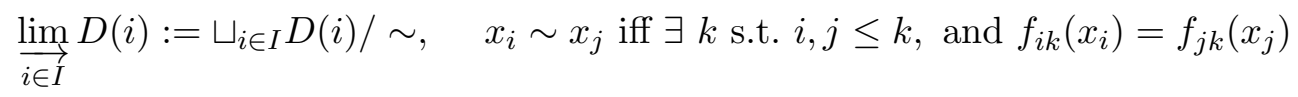

where $f_{i j}:=D(i \rightarrow j)$ for all $i \leq j$. We will sometimes write $\lim _{\rightarrow} D(i)$ for such a direct limit.

Definition 2.34. The inverse limit of an inverse system $V: K \longrightarrow R$-mod is defined to be the graded $R$-module $\lim _{\longleftarrow} V$ where

$$
\left(\lim _{\longleftarrow} V\right)_{p}:=\left\{\left(x_{k}\right)_{k \in K} \in \prod_{k \in K}(V(k))_{p}: \forall k, \check{k} \in K \text { s.t. } k \leq_{K} \check{k}, f_{k \check{k}}\left(x_{k}\right)=x_{\check{k}}\right\}
$$

for each $p \in \mathbb{Z}$ where $f_{k \breve{k}}:=V(k \rightarrow \check{k})$ for all $k \leq \check{k}$. We will sometimes write $\lim _{j} V(j)$ for such an inverse limit.

We now wish to describe an appropriate category of directed/inverse/double systems. This category should have the property that $\lim _{\mathrm{i}}$ and $\mathrm{lim}_{\longleftarrow}$ are functors and so that certain "obvious" endomorphisms of directed/inverse/double systems inducing isomorphisms on $\underline{\lim }$ and $\underset{\longleftarrow}{\mathfrak{l i m}}$ are 
in fact isomorphisms in this category. Another important property of this category is that we require a "derived" version of $\lim _{\longleftarrow}$ to exist.

Definition 2.35. Let $W: I \times K \longrightarrow R$-mod be a double system and let $\phi: W(i, k) \longrightarrow$ $A, \phi^{\prime}: A^{\prime} \longrightarrow W\left(i^{\prime}, k^{\prime}\right)$ be morphisms in $R$-mod for some $i, i^{\prime} \in I, k, k^{\prime} \in K$ satisfying $\left(i^{\prime}, k^{\prime}\right) \leq_{I \times K}(i, k)$. Then we define the $W$-composition $\phi \circ_{W} \phi^{\prime}: A^{\prime} \longrightarrow A$ of $\phi^{\prime}$ and $\phi$ to be the composition $\phi \circ f_{i^{\prime} k^{\prime} i k} \circ \phi^{\prime}$ where $f_{i^{\prime} k^{\prime} i k}:=W\left(\left(i^{\prime}, k^{\prime}\right) \rightarrow(i, k)\right)$.

Definition 2.36. Let $W_{j}: I_{j} \times K_{j} \longrightarrow R$-mod, $j=0,1$ be double systems. Then we can define a new double system $\mathcal{M} \operatorname{cor}\left(W_{0}, W_{1}\right):\left(I_{1} \times K_{0}^{\mathrm{op}}\right) \times\left(I_{0}^{\mathrm{op}} \times K_{1}\right) \longrightarrow R$-mod where the object $\left(\left(i_{1}, k_{0}\right),\left(i_{0}, k_{1}\right)\right)$ is sent to $\operatorname{Hom}_{R-\bmod }\left(W_{0}\left(i_{0}, k_{0}\right), W_{1}\left(i_{1}, k_{1}\right)\right)$ and a morphism $\left(\left(i_{1}, k_{0}\right),\left(i_{0}, k_{1}\right)\right) \rightarrow\left(\left(i_{1}^{\prime}, k_{0}^{\prime}\right),\left(i_{0}^{\prime}, k_{1}^{\prime}\right)\right)$ is sent to the morphism

$$
\begin{gathered}
\operatorname{Hom}_{R \text {-mod }}\left(W_{0}\left(i_{0}, k_{0}\right), W_{1}\left(i_{1}, k_{1}\right)\right) \longrightarrow \operatorname{Hom}_{R-\bmod }\left(W_{0}\left(i_{0}^{\prime}, k_{0}^{\prime}\right), W_{1}\left(i_{1}^{\prime}, k_{1}^{\prime}\right)\right) \\
\Phi \longrightarrow f_{i_{1} k_{1} i_{1}^{\prime} k_{1}^{\prime}}^{1} \Phi \circ f_{i_{0}^{\prime} k_{0}^{\prime} i_{0} k_{0}}^{0}
\end{gathered}
$$

where $f_{i k i^{\prime} k^{\prime}}^{j}:=W_{j}\left((i, k) \rightarrow\left(i^{\prime}, k^{\prime}\right)\right)$ for all $(i, k) \leq_{I_{j} \times K_{j}}\left(i^{\prime}, k^{\prime}\right), j=0,1$. We define

$$
\operatorname{Mor}\left(W_{0}, W_{1}\right):={\underset{i}{i_{0}}}_{\lim _{i_{1}}}^{\lim } \underset{k_{1}}{\lim } \underset{k_{0}}{\lim } \mathcal{M} \operatorname{Tor}\left(W_{0}, W_{1}\right)\left(\left(i_{1}, k_{0}\right),\left(i_{0}, k_{1}\right)\right) \text {. }
$$

We define the category of double systems 2-sys- $R$ to be the category whose objects are double systems, whose morphisms between objects $W_{0}$ and $W_{1}$ are elements of $\operatorname{Mor}\left(W_{0}, W_{1}\right)$ and where composition is induced by composition of $R$-module morphisms in the following way. If $W_{j}: I_{j} \times K_{j} \longrightarrow R$-mod, $j=0,1,2$ are double systems then since inverse and direct limits commute with finite products, we can define the composition maps as follows:

$$
\begin{aligned}
& \operatorname{Mor}\left(W_{1}, W_{2}\right) \times \operatorname{Mor}\left(W_{0}, W_{1}\right)= \\
& \underbrace{\lim }_{i_{0}} \underset{i_{1}^{\prime}}{\lim } \underset{i_{1}}{\lim } \underset{i_{2}}{\lim } \underset{k_{1}}{\lim _{k_{2}}} \underset{\lim _{k_{0}}}{\lim } \underset{k_{1}^{\prime}}{\lim } \operatorname{Hom}\left(W_{1}\left(i_{1}^{\prime}, k_{1}^{\prime}\right), W_{2}\left(i_{2}, k_{2}\right)\right) \times \operatorname{Hom}\left(W_{0}\left(i_{0}, k_{0}\right), W_{1}\left(i_{1}, k_{1}\right)\right)
\end{aligned}
$$

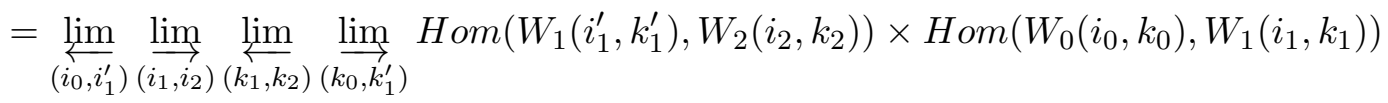

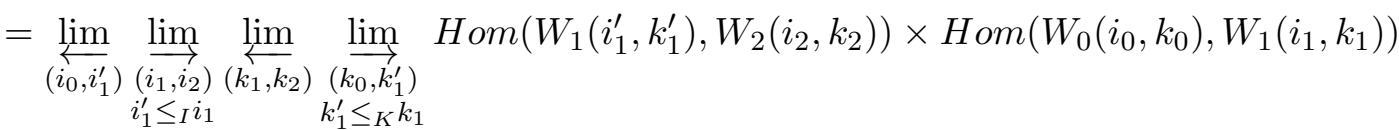

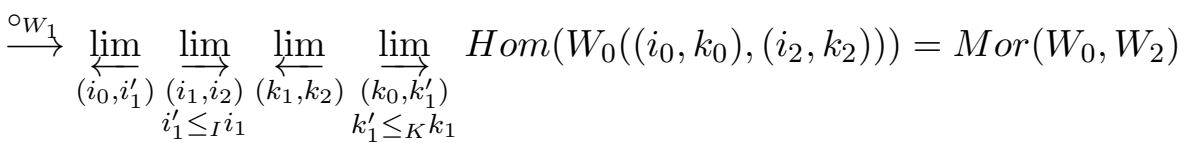

where Hom $:=H_{\text {om }}$-mod. The category Ind-R of directed systems is the full subcategory of 2 -sys- $R$ whose objects are directed systems. Similarly the category Pro- $R$ of inverse systems is the full subcategory whose objects are inverse systems.

Example 2.37. A key example of a morphism $\phi \in \operatorname{Mor}\left(W_{0}, W_{1}\right)$ of double systems $W_{j}$ : $I_{j} \times K_{j} \longrightarrow R$-mod, $j=0,1$ is a natural transformation $\phi$ from $W_{0} \circ\left(\operatorname{id}_{I_{0}} \times F\right)$ to $W_{1} \circ\left(G \times \mathrm{id}_{K_{1}}\right)$ where $F: K_{1} \longrightarrow K_{0}$ and $G: I_{0} \longrightarrow I_{1}$ are functors.

If $I_{0}=I_{1}$ and $K_{0}=K_{1}$ and the natural transformation maps are double system maps, then such a morphism is equal to the identity map in 2 -sys- $R$. We will call such a natural transformation a standard endomorphism.

Suppose $\phi^{\prime} \in \operatorname{Mor}\left(W_{1}, W_{2}\right)$ is another morphism where $W_{2}: I_{2} \times K_{2} \longrightarrow R$-mod is a double system and where it is also given by a natural transformation $\phi^{\prime}: W_{1} \circ\left(\mathrm{id}_{I_{1}} \times F^{\prime}\right)$ to 
$W_{2} \circ\left(G^{\prime} \times \operatorname{id}_{K_{2}}\right)$ where $F^{\prime}: K_{2} \longrightarrow K_{1}$ and $G: I_{1} \longrightarrow I_{2}$ are functors. Then the composition $\phi^{\prime} \circ \phi \in \operatorname{Mor}\left(W_{0}, W_{2}\right)$ can be represented by the natural transformation

$$
\left(\phi^{\prime} \cdot\left(\operatorname{id}_{G \times \operatorname{id}_{K_{2}}}\right)\right) \circ\left(\phi \cdot\left(\operatorname{id}_{\operatorname{id}_{I_{0}} \times F^{\prime}}\right)\right): W_{0} \circ\left(\operatorname{id}_{I_{0}} \times F^{\prime}\right) \longrightarrow W_{2} \circ\left(G \times \operatorname{id}_{K_{2}}\right)
$$

where $\circ$ denotes vertical composition of natural transformations and d denotes horizontal composition and where $\operatorname{id}_{Q}$ means the identity natural transformation from a functor $Q$ to itself. Here we have also used the identity $\left(G \times \mathrm{id}_{K_{1}}\right) \circ\left(\operatorname{id}_{I_{0}} \times F^{\prime}\right)=\left(\operatorname{id}_{I_{1}} \times F^{\prime}\right) \circ\left(G \times \operatorname{id}_{K_{2}}\right)$.

Definition 2.38. A cofinal subsystem of a double system $W: I \times K \longrightarrow R$-mod is the restriction $\check{W}:=\left.W\right|_{\check{I} \times \check{K}}$ of $W$ to the subcategory $\check{I} \times \check{K} \subset I \times K$ where $\check{I} \subset I, \check{K}^{\text {op }} \subset K^{\text {op }}$ are cofinal subsets. The inclusion morphism is the morphism $\iota_{\breve{W}, W}: \check{W} \longrightarrow W$ in 2 -sys- $R$ given by the natural transformation between $\check{W} \circ\left(\operatorname{id}_{\check{I}} \times F\right)$ to $W \circ\left(G \times \mathrm{id}_{K}\right)$ constructed using double system maps where $F: K \longrightarrow \check{K}$ satisfies $k \leq_{K} F(k)$ for all $k \in K$ and where $G: \check{I} \longrightarrow I$ is the natural inclusion map. Here $F$ is constructed using the axiom of choice.

Remark 2.39. The inclusion map does not depend on the choice of functor $F$. This due to the fact that if we have a morphism $\iota_{\breve{W}, W}^{\prime}: \check{W} \longrightarrow W$ constructed in the same way, but using a different choice of functor $F$ then there are standard endomorphisms $E, E^{\prime}: W \longrightarrow W$ so that $E \circ \iota_{\breve{W}, W}=E^{\prime} \circ \iota_{\breve{W}, W}^{\prime}$. Since standard endomorphisms represent the identity map in 2-sys- $R$ we get that $\iota_{\breve{W}, W}=\iota_{\breve{W}, W}^{\prime}$.

Lemma 2.40. The inclusion map $\iota_{\breve{W}, W}$ from Definition 2.38 is an isomorphism.

Proof of Lemma 2.40. The inverse $\eta$ of $\iota_{\breve{W}, W}$ is given by the natural transformation between $W \circ\left(\operatorname{id}_{I} \times F^{\prime}\right)$ to $\check{W} \circ\left(G^{\prime} \times \operatorname{id}_{K}\right)$ built from double system maps where $F^{\prime}: \check{K} \longrightarrow K$ is the natural inclusion map and where $G^{\prime}: I \longrightarrow \check{I}$ satisfies $i \leq_{I} F(i)$ for all $i \in I$. The compositions $\eta \circ \iota_{\breve{W}, W}, \iota_{\breve{W}, W} \circ \eta$ as described in Example 2.37 are standard endomorphisms and hence $\iota_{\breve{W}, W}$ is an isomorphism.

Definition 2.41. We define $\stackrel{\lim }{\longrightarrow} \underset{\lim }{\longleftarrow}$ to be the functor

$$
\underset{\lim }{\longrightarrow} \lim : 2 \text {-sys- } R \longrightarrow R \text {-mod, } \quad \stackrel{\lim \lim }{\longleftarrow}:=\oplus_{m \in \mathbb{Z}} \operatorname{Hom}_{2 \text {-sys- } R}\left(\iota_{R}[-m],-\right)
$$

where $\iota_{R}$ is the trivial double system. We define $\lim _{\longrightarrow}:$ Ind- $R \longrightarrow R$-mod (resp. $\lim ^{\prime}$ Pro- $R \longrightarrow R$-mod) to be the restriction of this functor to the subcategory Ind- $R$ (resp. Pro- $R$ ).

Remark 2.42. The functor $\lim \lim$ sends a double system $(W(i, k))_{(i, k) \in I \times K}$ to the limit $\lim _{i} \lim _{k} W(i, k)$. Similarly the functors $\underline{\lim }$ and $\lim _{\longleftarrow}$ above coincide with $\underline{\lim }$ and $\underset{\longleftarrow}{\lim }$ from Definitions 2.33 and 2.34.

Definition 2.43. Let $W_{j}: I_{j} \times K_{j} \longrightarrow R$-mod, $j=0,1$ be a double systems. We define $W_{0} \times W_{1}$ to be the double system $\left(W_{0}(i, k) \times W_{1}(\check{i}, \check{k})\right)_{(i, \check{i}, k, \breve{k}) \in\left(I_{0} \times I_{1}\right) \times\left(K_{0} \times K_{1}\right)}$ with the natural double system maps induced from the double system maps of $W_{j}, j=0,1$. We define $W_{0} \otimes W_{1}$ to be the double system $\left(W_{0}(i, k) \otimes_{R} W_{1}(\check{i}, \check{k})\right)_{(i, \check{i}, k, \breve{k}) \in\left(I_{0} \times I_{1}\right) \times\left(K_{0} \times K_{1}\right)}$ with the natural double system maps induced from the double system maps of $W_{j}, j=0,1$.

Let $\check{W}_{j}: \check{I}_{j} \times \check{K}_{j} \longrightarrow R$-mod, $j=0,1$ be two additional double systems and let $T_{W_{0}, W_{1}, \breve{W}_{0}, \breve{W}_{1}}$ be the natural composition

$$
\begin{aligned}
& T_{W_{0}, W_{1}, \check{W}_{0}, \check{W}_{1}}: \operatorname{Mor}_{2 \text {-sys- }}\left(W_{0}, \check{W}_{0}\right) \otimes_{R} \operatorname{Mor}_{2 \text {-sys- } R}\left(W_{1}, \check{W}_{1}\right)= \\
&(\underbrace{\lim }_{i_{0}} \underset{\breve{i}_{0}}{\lim } \underbrace{\lim }_{\check{k}_{0}} \underset{k_{0}}{\lim } \operatorname{Hom}\left(W_{0}\left(i_{0}, k_{0}\right), \check{W}_{0}\left(\check{i}_{0}, \check{k}_{0}\right)\right)) \otimes_{R}(\underbrace{\lim }_{i_{1}} \underset{\breve{i}_{1}}{\lim } \underbrace{\lim }_{\check{k}_{1}} \underset{k_{1}}{\lim } \operatorname{Hom}\left(W_{1}\left(i_{1}, k_{1}\right), \check{W}_{1}\left(\check{i}_{1}, \check{k}_{1}\right)\right))
\end{aligned}
$$




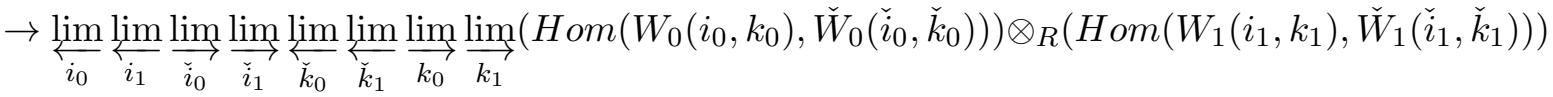

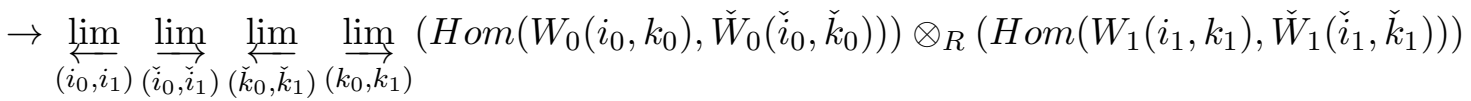

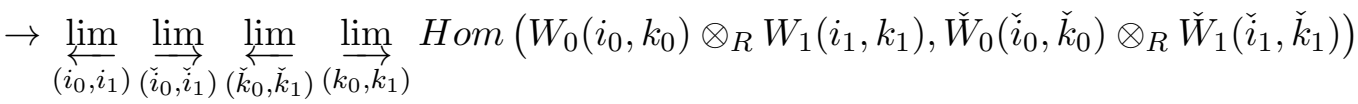

$$
\begin{aligned}
& =\operatorname{Mor}\left(W_{0} \otimes W_{1}, \check{W}_{0} \otimes \check{W}_{1}\right)
\end{aligned}
$$

where $H o m=H_{o m}$-mod. For any two morphisms $\Phi_{j}: W_{j} \longrightarrow \check{W}_{j}, j=0,1$ in 2-sys- $R$, we define $\Phi_{0} \otimes \Phi_{1}:=T_{W_{0}, W_{1}, \breve{W}_{0}, \check{W}_{1}}\left(\Phi_{0} \otimes_{R} \Phi_{1}\right) \in M_{\text {or }}{ }_{2 \text {-sys- } R}\left(W_{0} \otimes W_{1}, \check{W}_{0} \otimes \check{W}_{1}\right)$.

Also, if $W: I \times K \longrightarrow R$-mod is a double system, we define $\tau_{W}: W \otimes W \longrightarrow W \otimes W$ to be the morphism sending $x \otimes y$ to $(-1)^{p q} y \otimes x$ for all $x \in(W(i, k))_{p}, y \in\left(W\left(i^{\prime}, k^{\prime}\right)\right)_{q},(i, k)$, $\left(i^{\prime}, k^{\prime}\right)$ in $I \times K$ and $p, q \in \mathbb{Z}$.

These operations make 2-sys- $R$ into a symmetric monoidal category together with the identity object $\iota_{R}$ due to the fact that $R$-mod is a symmetric monoidal category and the fact that natural transformations $\Phi$ between double systems $W_{j}: I \times K \longrightarrow R$-mod, $j=0,1$ where the morphisms of $\Phi$ are isomorphisms induce isomorphisms in 2-sys- $R$.

Definition 2.44. A product on $W: I \times K \longrightarrow R$-mod is a morphism $\mu: W \otimes W \longrightarrow W$ so that $\mu \circ\left(\operatorname{id}_{W} \otimes \mu\right)=\mu \circ\left(\mu \otimes \operatorname{id}_{W}\right)$ using the natural identification $(W \otimes W) \otimes W \cong W \otimes(W \otimes W)$. Such a product is graded commutative if $\mu \circ \tau=\mu$. The product $\mu$ is unitary if there exists a morphism $\iota: \iota_{R} \longrightarrow W$ where $\iota_{R}$ is the trivial double system satisfying $\mu \circ\left(\operatorname{id}_{W} \otimes \iota\right)=$ $\mu \circ\left(\iota \circ \operatorname{id}_{W}\right)=\operatorname{id}_{W}$ where we identify $W$ with $W \otimes \iota_{R}$ and $\iota_{R} \otimes W$ in the natural way. A morphism of double systems $W_{0}, W_{1}$ with products $\mu_{j}: W_{j} \otimes W_{j} \longrightarrow W_{j}, j=0,1$ is a morphism $\Phi: W_{0} \longrightarrow W_{1}$ satisfying $\Phi \circ \mu_{0}=\mu_{1} \circ(\Phi \otimes \Phi)$. A (graded commutative) (unital) product on a directed or inverse system is a (graded commutative) (unital) product on the corresponding double system.

Remark 2.45. From now on, all products on double systems (resp. directed/inverse systems) will be unital graded commutative products. Hence from now on, we will just call them products. We will also assume all morphisms between such double systems with products preserve the unit. If $W$ is a double system with product $\mu: W \otimes W \longrightarrow W$, then we get a product

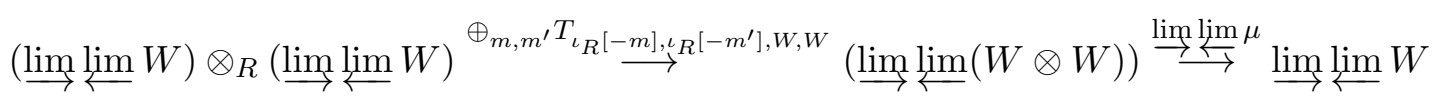

on $\underline{\lim } \underset{\lim }{\longleftarrow} W$ making it into a unital graded commutative $R$-algebra where $T_{\iota_{R}[-m], \iota_{R}\left[-. m^{\prime}\right], W, W}$ is given in Definition 2.43 and where we use the natural identification $\iota_{R} \cong \iota_{R} \otimes \iota_{R}$. Similarly a product on a directed/inverse system gives us an induced product on its direct/inverse limit.

We will also need to show that "derived" versions of the functor $\lim _{\longrightarrow} \lim$ exist. We need some preliminary definitions and lemmas before we define such a functor.

Definition 2.46. Let $F:$ Pro- $R \longrightarrow R$-mod be a functor so that the corresponding maps $F: \operatorname{Mor}_{\text {Pro- } R}\left(P_{0}, P_{1}\right) \longrightarrow$ Mor $_{R \text {-mod }}\left(F\left(P_{0}\right), F\left(P_{1}\right)\right)$ are $R$-module maps for each pair of objects $P_{0}, P_{1}$ in Pro- $(R)$. We define the functor $\lim _{\longrightarrow} F: 2$-sys- $R \longrightarrow R$-mod as follows. If $W$ : $I \times K \longrightarrow R$-mod is a double system then $\lim _{\longrightarrow} F(W):=\underline{\lim }_{i} F\left(\left.W\right|_{i}\right)$ where $\left.W\right|_{i}$ is the inverse 
system $\left(W_{i, k}\right)_{k \in K}$. Also if $W_{j}: I_{j} \times K_{j} \longrightarrow R$-mod, $j=0,1$ are double systems, the corresponding functor on morphisms is the natural composition:

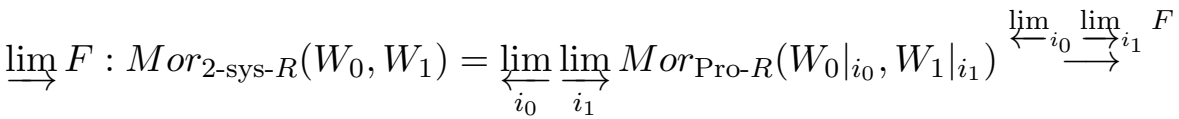

$$
\begin{aligned}
& \left.\underset{i_{0}}{\lim _{i_{1}}} \lim _{R} \operatorname{Mor}_{R-\bmod }\left(F\left(\left.W_{0}\right|_{i_{0}}\right), F\left(\left.W_{1}\right|_{i_{1}}\right)\right)=M_{\text {or }} \operatorname{Ind}-R_{(}\left(F\left(\left.W_{0}\right|_{i}\right)\right)_{i \in I_{0}},\left(F\left(\left.W_{1}\right|_{i}\right)\right)_{i \in I_{1}}\right) \\
& \stackrel{\lim }{\longrightarrow} \operatorname{Mor}_{R-\bmod }\left(\underset{i_{0}}{\lim } F\left(\left.W_{0}\right|_{i_{0}}\right), \underset{i_{1}}{\lim } F\left(\left.W_{1}\right|_{i_{1}}\right)\right) .
\end{aligned}
$$

Definition 2.47. Let $\left(K, \leq_{K}\right)$ be an inverse directed set. Define $R$-mod ${ }^{K}$ to be the category whose objects are inverse systems $V: K \longrightarrow R$-mod and whose morphisms are natural transformations between such objects. We define $\alpha_{K}: R$-mod ${ }^{K} \longrightarrow 2$-sys- $R$ to be the functor sending objects $V$ to $V$ and sending morphisms to the induced morphisms in 2-sys- $R$.

Since the category $R$-mod ${ }^{K}$ has enough injectives by [Mar00, Theorem 11.18] we have the following definition below. Technically [Mar00, Theorem 11.18] proves this for ungraded modules, but the graded case follows immediately since a graded module is a direct sum of ungraded ones. This will also be true for other theorems cited in [Mar00].

Definition 2.48. Let $\left(K, \leq_{K}\right)$ be an inverse directed set. For each $k \in \mathbb{N}$, define $\left.\varliminf^{k}\right|_{K}$ : $R$-mod ${ }^{K} \longrightarrow R$-mod to be the $k$ th right derived functor of $\varliminf_{\longleftarrow} \circ \alpha_{K}$.

The following lemma follows immediately from Theorem 15.5 and Remark 15.6 in [Mar00].

Lemma 2.49. There is a natural functor $\lim ^{k}:$ Pro- $R \longrightarrow R$-mod satisfying $\lim ^{k} \circ \alpha_{K}=$ $\left.\lim ^{k}\right|_{K}$ for all inverse directed sets $\left(K, \leq_{K}\right)$.

For our purposes, it does not matter how the functor $\lim ^{k}$ is constructed. We will only use the fact that it satisfies the property stated in the lemma above.

Definition 2.50. For each $k \in \mathbb{N}$, define the direct limit of $\lim ^{k}$ to be the functor $\underset{\lim }{\longrightarrow} \lim ^{k}$ : 2 -sys- $R \longrightarrow R$-mod where $\stackrel{\lim }{\longrightarrow}$ is given as in Definition 2.46 and $\varliminf^{k}$ is constructed in Lemma 2.49 .

Finally, we need a test telling us when $\lim _{\longrightarrow} \lim ^{1}$ vanishes.

Lemma 2.51. Suppose that $W: I \times K \longrightarrow R$-mod is a double system where $\left(I, \leq_{I}\right)$ (resp. $\left.\left(K, \leq_{K}\right)\right)$ is a directed (resp. inverse directed) set. Suppose that there is a cofinal family $\check{I} \subset I$ and a countably infinite cofinal family $\check{K} \subset K$ of $\left(K, \leq_{K}\right)^{\text {op }}$ with the property that for all $i \in \check{I}, k, k^{\prime} \in \check{K}$ satisfying $k \leq_{K} k^{\prime}$ we have that $W\left((i, k) \longrightarrow\left(i, k^{\prime}\right)\right)$ is surjective. Then

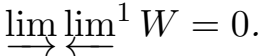

Proof of Lemma 2.51. Let $\left.W\right|_{i}$ be the inverse system $(W(i, k))_{k \in K}$ for each $i \in I$ and let $\left.\check{W}\right|_{i}$ be the inverse system $(W(i, k))_{k \in \check{K}}$. Since $\check{K}$ is countably infinite, we can assume after passing to a cofinal subset of $\check{K}$ that $\left(\check{K}, \leq_{K}\right)$ is equal to the inverse directed set $\left(\mathbb{N}^{\text {op }}, \leq^{\text {op }}\right)$ by Lemma 2.40. By [Wei94, Proposition 3.5.7] combined with [Mar00, Lemma 11.49] we have that $\left.\lim ^{1} \breve{W}\right|_{i}=0$ for each $i \in \check{I}$. Hence by [Mar00, Theorem 14.9] we have that $\left.\lim ^{1} W\right|_{i}=0$ for each $i \in \check{I}$. Hence $\underset{\lim }{\longrightarrow} \lim ^{1} W=0$. 
2.4. Novikov Rings. In this section we give a definition of a Novikov ring (which we will define using inverse and direct limits). Novikov rings are appropriate coefficient rings for our Hamiltonian Floer cohomology groups. Throughout this subsection we will fix a (possibly empty) contact cylinder $\check{C}=[1-\epsilon, 1+\epsilon] \times C$ and we will let $D \subset M$ be its associated Liouville domain.

Definition 2.52. Let $W$ be a finite dimensional real vector space. A convex cone is a subset $Q \subset W$ so that for all $x, y \in Q$ and all positive real numbers $\alpha, \beta>0$, we have that $\alpha x+\beta y \in Q$. Such a cone is called salient if, for each $x \in Q-0,-x \notin Q$. Now suppose $(A, \cdot)$ is a finitely generated abelian group and let $Q$ be a cone in $\left(A \otimes_{\mathbb{Z}} \mathbb{R}\right)^{*}$. Define $\preceq_{Q}$ to be the binary relation on $A$ where $x \preceq_{Q} y$ if and only if $f\left(\left(y \cdot x^{-1}\right) \otimes 1\right) \geq 0$ for all $f \in Q$.

Remark 2.53. If $Q$ is a closed salient cone, then $\left(A, \preceq_{Q}\right)$ and $\left(A^{\mathrm{op}}, \preceq_{Q}^{\mathrm{op}}\right)$ are directed sets.

Definition 2.54. Let $(A, \cdot)$ be a finitely generated abelian group and let $Q \subset\left(A \otimes_{\mathbb{Z}} \mathbb{R}\right)^{*}$ be a closed salient cone. For each $x \in A$, let $F_{x}^{Q}$ be the free $\mathbb{K}$-module generated by elements of the set $S_{x}^{Q}:=\left\{a \in A: x \preceq_{Q} a\right\}$. Let $\left(I, \leq_{I}\right)$ and be the (inverse) directed set $\left(A^{\mathrm{op}}, \preceq_{Q}^{\mathrm{op}}\right)$. Then $F^{Q}:=\left(F_{x_{-}, x_{+}}^{Q}\right)_{\left(x_{-}, x_{+}\right) \in I \times I}$ is a double system where $F_{x_{-}, x_{+}}^{Q}:=F_{x_{-}}^{Q} /\left(F_{x_{-}}^{Q} \cap F_{x_{+}}^{Q}\right)$ and where the double system maps are the natural compositions

$$
F_{x_{-}, x_{+}}^{Q} \longrightarrow F_{x_{-}, x_{+}^{\prime}}^{Q} \longrightarrow F_{x_{-}^{\prime}, x_{+}^{\prime}}^{Q}
$$

for all $x_{ \pm}, x_{ \pm}^{\prime} \in A$ satisfying $x_{ \pm}^{\prime} \preceq_{Q} x_{ \pm}$. Choose a function $M: A^{2} \longrightarrow A$ satisfying $M(x, y) \preceq_{Q} x$ and $M(x, y) \preceq_{Q} y$ for all $(x, y) \in A^{2}$. For each $x_{-}^{0}, x_{-}^{1}, x_{+}^{2} \in A$, let

$$
\mu: F_{x_{-}^{0}, x_{+}^{2}-x_{-}^{1}}^{Q} \otimes_{\mathbb{K}} F_{x_{-}^{1}, x_{+}^{2}-x_{-}^{0}}^{Q} \longrightarrow F_{x_{-}^{0} \cdot x_{-}^{1}, x_{+}^{2}}^{Q}
$$

be the unique $\mathbb{K}$-linear map sending $\left[a_{0}\right] \otimes\left[a_{1}\right]$ to $\left[a_{0} \cdot a_{1}\right]$ for all $a_{j} \in S_{x_{-}^{j}}^{Q}, j=0,1$. Then $\mu$ defines for us a product $\mu: F^{Q} \otimes F^{Q} \longrightarrow F^{Q}$ on the double system $F^{Q}$. We define the $(A, Q)$-Novikov ring to be the ring

$$
\Lambda_{\mathbb{K}}^{A, Q}:=\underset{x_{-} \in I}{\lim } \underset{x_{+} \in I}{\lim _{x_{-}, x_{+}}} F^{Q}
$$

whose product is induced by $\mu$ as in Remark 2.45. We also have a subring

$$
\Lambda_{\mathbb{K}}^{A, Q,+}:=\lim _{x_{+} \in I} F_{0, x_{+}}^{Q} \subset \Lambda_{\mathbb{K}}^{A, Q}
$$

called the positive $(A, Q)$-Novikov ring.

Remark 2.55. We can think of $\Lambda^{A, Q}$ as the ring of formal power series

$$
\Lambda_{\mathbb{K}}^{A, Q}=\left\{\sum_{i \in \mathbb{N}} b_{i} t^{a_{i}}: b_{i} \in \mathbb{K}, a_{i} \in A \quad \forall i \in \mathbb{N},\left(a_{i}\right)_{i \in \mathbb{N}} \text { is cofinal in }\left(A, \preceq_{Q}\right)\right\} .
$$

Intuitively, the terms in this series must "tend to infinity" in the "cone" $\left\{x \in A: 0 \preceq_{Q} x\right\}$.

Definition 2.56. Let $\iota_{D}: H^{2}(M, D ; \mathbb{R}) \longrightarrow H^{2}(M ; \mathbb{R})$ be the natural restriction map on cohomology and let $\pi_{D}: H^{2}(M, D ; \mathbb{R}) \times \mathbb{R} \times \mathbb{R} \longrightarrow H^{2}(M, D ; \mathbb{R})$ be the natural projection map. Let $Q \subset H^{2}(M, D ; \mathbb{R}) \times \mathbb{R} \times \mathbb{R}$ be a cone so that $\check{Q}:=\iota_{D}\left(\pi_{D}(Q)\right)$ is a closed and salient cone. Using the canonical identification

$$
\left(H_{2}(M ; \mathbb{Z}) \otimes_{\mathbb{Z}} \mathbb{R}\right)^{*}=H^{2}(M ; \mathbb{R}),
$$


we can define the $Q$-Novikov ring to be the $\left(H_{2}(M ; \mathbb{Z}), \check{Q}\right)$-Novikov ring

$$
\Lambda_{\mathbb{K}}^{Q}:=\Lambda_{\mathbb{K}}^{H_{2}(M ; \mathbb{Z}), \check{Q}}
$$

and the positive $Q$-Novikov ring to be the positive $\left(H_{2}(M ; \mathbb{Z}), \check{Q}\right)$-Novikov ring

$$
\Lambda_{\mathbb{K}}^{Q,+}:=\Lambda_{\mathbb{K}}^{H_{2}(M ; \mathbb{Z}), \check{Q},+} .
$$

The Novikov rings above are designed to deal with multiple action values encoded in Definition 2.10. Note that we could have defined the above Novikov ring $\Lambda_{\mathbb{K}}^{Q}$ to be a $\left(H_{2}(M, D ; \mathbb{Z}) \times \mathbb{Z} \times \mathbb{Z}, Q\right)$-Novikov ring associated to $Q$ instead. Such a definition would have forced us to generalize the notion of a capped 1-periodic orbit so that the "capping" also has some boundary components inside $D$ (one would have to modify the way action is calculated too and restrict the class of Hamiltonians further). This would have given us more information, but would have added an extra layer of unnecessary complication. Therefore we have decided to use this simpler definition.

Example 2.57. Let $\widetilde{\omega}_{\#}=\left(\widetilde{\omega}_{i}\right)_{i \in S}$ be a finite collection of $\check{C}$-compatible 2-forms with scaling constants $\left(\lambda_{i}^{ \pm}\right)_{i \in S}$ and let $\omega_{\check{C}}$ be a $\check{C}$-compatible 2 -form with scaling constants 0 and 1 and which is equal to $\omega$ outside $D \cup([1,1+\epsilon / 2] \times C)$. Let $Q_{\widetilde{\omega}_{\#}} \subset H^{2}(M, D ; \mathbb{R}) \times \mathbb{R} \times \mathbb{R}$ be the smallest convex cone containing $\left(\left[\widetilde{\omega}_{i}-\lambda_{i}^{-} \omega+\lambda_{i}^{-} \omega_{\check{C}}\right], \lambda_{i}^{-}, \lambda_{i}^{+}\right) \in H^{2}(M, D ; \mathbb{R}) \times \mathbb{R} \times \mathbb{R}$ for each $i \in S$. The $\left(\widetilde{\omega}_{\#}\right)$-Novikov ring is the Novikov ring:

$$
\Lambda_{\mathbb{K}}^{\widetilde{\omega}_{\#}}:=\Lambda_{\mathbb{K}}^{Q_{\widetilde{\omega}_{\#}}} .
$$

The two key examples for this paper are when

- $\widetilde{\omega}_{\#}$ has one element $\widetilde{\omega}$, giving us a Novikov ring $\Lambda_{\mathbb{K}}^{\widetilde{\omega}}:=\Lambda_{\mathbb{K}}^{\widetilde{\omega}_{\#}}$, which can be thought of as the set of power series

$$
\Lambda_{\mathbb{K}}^{\widetilde{\omega}}=\left\{\sum_{i \in \mathbb{N}} b_{i} t^{a_{i}}: b_{i} \in \mathbb{K}, a_{i} \in H_{2}(M ; \mathbb{Z}), \quad \widetilde{\omega}\left(a_{i}\right) \rightarrow \infty\right\}
$$

- and when $\widetilde{\omega}_{\#}$ has two elements $\widetilde{\omega}_{0}, \widetilde{\omega}_{1}$, giving us a Novikov ring $\Lambda_{\mathbb{K}}^{\widetilde{\omega}_{0}, \widetilde{\omega}_{1}}:=\Lambda_{\mathbb{K}}^{\widetilde{\omega}_{\#}}$ which can be thought of as the set of power series

$$
\Lambda_{\mathbb{K}}^{\widetilde{\omega}_{0}, \widetilde{\omega}_{1}}=\left\{\sum_{i \in \mathbb{N}} b_{i} t^{a_{i}}: b_{i} \in \mathbb{K}, a_{i} \in H_{2}(M ; \mathbb{Z}), \quad \min \left(\widetilde{\omega}_{0}\left(a_{i}\right), \widetilde{\omega}_{1}\left(a_{i}\right)\right) \rightarrow \infty\right\} .
$$

2.5. Definition of Floer Cohomology using Alternative Filtrations. In this section we will give a definition of Hamiltonian Floer cohomology using the action function in Definition 2.10. Throughout this subsection, we will fix a (possibly empty) contact cylinder $\check{C}=[1-$ $\epsilon, 1+\epsilon] \times C \subset M$ with associated Liouville domain $D$ and cylindrical coordinate $r_{C}$. We also let $\iota_{D}: H^{2}(M, D ; \mathbb{R}) \longrightarrow H^{2}(M ; \mathbb{R})$ be the natural restriction map on cohomology and let $\pi_{D}: H^{2}(M, D ; \mathbb{R}) \times \mathbb{R} \times \mathbb{R} \longrightarrow H^{2}(M, D ; \mathbb{R})$ be the natural projection map.

In order to define Hamiltonian Floer cohomology with the right properties, we need to consider certain cones inside $H^{2}(M, D ; \mathbb{R}) \times \mathbb{R} \times \mathbb{R}$.

Definition 2.58. Let $\omega_{\check{C}}$ be a $\check{C}$-compatible 2 -form with scaling constants 0 and 1 and which is equal to $\omega$ outside $D \cup([1,1+\epsilon / 2] \times C)$. A cone $Q \subset H^{2}(M, D ; \mathbb{R}) \times \mathbb{R} \times \mathbb{R}$ is $\check{C}$-compatible if

- both $\iota_{D}\left(\pi_{D}(Q)\right)$ and $Q$ are closed and salient and

- $Q \subset Q_{\check{C}} \cup\{0\}$ where $Q_{\check{C}}$ is given as in Definition 2.10. 
A $\check{C}$-compatible cone $Q$ is called thin if $\left.\pi_{D}\right|_{Q}: Q \longrightarrow H^{2}(M, D ; \mathbb{R})$ is an injective map. A $\check{C}$-compatible cone $Q$ is called small if $Q \subset \mathbb{R}\left[\omega_{\check{C}}\right] \times \mathbb{R} \times \mathbb{R}$ and if the natural projection map

$$
\mathbb{R}\left[\omega_{\check{C}}\right] \times \mathbb{R} \times \mathbb{R} \longrightarrow \mathbb{R}\left[\omega_{\check{C}}\right] \times \mathbb{R}, \quad\left(q, \lambda_{-}, \lambda_{+}\right) \longrightarrow\left(q, \lambda_{-}\right)
$$

restricted to $Q$ is injective. A pair of $\check{C}$-compatible cones $\left(Q_{-}, Q_{+}\right)$is called wide if for each $q \in \pi_{D}\left(Q_{-}\right) \cup \pi_{D}\left(Q_{+}\right)$there exists $\lambda_{ \pm,-}^{q}, \lambda_{ \pm,+}^{q} \in \mathbb{R}$ so that $\left(q, \lambda_{ \pm,-}^{q}, \lambda_{ \pm,+}^{q}\right) \in Q_{ \pm}$,

$$
\lambda_{-,-}^{q}<\lambda_{+,-}^{q} \text { and } \lambda_{-,+}^{q}=\lambda_{+,+}^{q} .
$$

A $\check{C}$-interval domain pair is a pair $\left(Q_{-}, Q_{+}\right)$of $\check{C}$-compatible cones so that

(1) $Q_{+}$is thin and not equal to the trivial cone $\{0\}$,

(2) $Q_{+} \subset Q_{-}$and

(3) if $Q_{-}$is not small, then $\left(Q_{-}, Q_{+}\right)$is wide.

For any $\check{C}$-compatible cone $Q$, we define $\operatorname{Sc}(Q)$ to be the space of continuous functions $f: Q \longrightarrow \mathbb{R}$ satisfying $f(\sigma x)=\sigma f(x)$ for all $x \in Q$ and $\sigma \geq 0$ equipped with the $C_{\text {loc }^{-}}^{0}$ topology. A $\check{C}$-action interval is a pair $\left(a_{-}, a_{+}\right) \in \operatorname{Sc}\left(Q_{-}\right) \times \operatorname{Sc}\left(Q_{+}\right)$where $\left(Q_{-}, Q_{+}\right)$is a $\check{C}$-interval domain pair. We say that $\left(a_{-}, a_{+}\right)$is small if $Q_{-}$and $Q_{+}$are small and wide if $\left(Q_{-}, Q_{+}\right)$is wide.

For each subset $P \subset \mathbb{Z}$, weakly $\check{C}$-compatible Hamiltonian $H$ and $\check{C}$-action interval $\left(a_{-}, a_{+}\right) \in \operatorname{Sc}\left(Q_{-}\right) \times \operatorname{Sc}\left(Q_{+}\right)$, define $\Gamma_{\check{C}, a_{-}, a_{+}}^{P}(H)$ to be the set of capped 1-periodic orbits $\gamma$ of $H$ whose associated 1-periodic orbit is not contained in $[1+\epsilon / 8,1+\epsilon / 2] \times C$, whose index is in $P$ and satisfying

$$
a_{-} \leq\left.\mathcal{A}_{H, \breve{C}}(\gamma)\right|_{Q_{-}}, \quad a_{+} \not \leq\left.\mathcal{A}_{H, \breve{C}}(\gamma)\right|_{Q_{+}}
$$

where $\mathcal{A}_{H, \check{C}}$ is given in Definition 2.10 .

If $\left(a_{-}, a_{+}\right) \in \operatorname{Sc}\left(Q_{-}\right) \times \operatorname{Sc}\left(Q_{+}\right)$is wide then we define the Hamiltonian height of $\left(a_{-}, a_{+}\right)$ to be

$$
\sup \left\{\begin{array}{cc}
\operatorname{height}\left(a_{-}, a_{+}\right):= \\
\frac{a_{+}\left(x, \lambda_{+,-}, \lambda_{+,+}\right)-a_{-}\left(x, \lambda_{-,-}, \lambda_{-,+}\right)}{\left(x, \lambda_{ \pm,-}, \lambda_{ \pm,+}\right) \in Q_{ \pm},} \\
\hline \lambda_{+,-}-\lambda_{-,-} & \begin{array}{c}
\lambda_{-,-}<\lambda_{+,-} \\
\lambda_{-,+}=\lambda_{+,+}
\end{array}
\end{array}\right\} .
$$

For each $\check{C}$-action interval $\left(a_{-}, a_{+}\right)$and each manifold $\Sigma$, define

$$
\begin{array}{cl}
\mathcal{H}^{\Sigma}\left(\check{C}, a_{-}, a_{+}\right):= \\
\begin{cases}\mathcal{H}^{\Sigma}(\check{C}) & \text { if }\left(a_{-}, a_{+}\right) \text {is small } \\
\left\{H \in \overline{\mathcal{H}}^{\Sigma}(\check{C}): m_{H_{\sigma}}>\operatorname{height}\left(a_{-}, a_{+}\right) \forall \sigma \in \Sigma\right\} & \text { otherwise }\end{cases}
\end{array}
$$

where $\mathcal{H}^{\Sigma}(\check{C})$ and $\overline{\mathcal{H}}^{\Sigma}(\check{C})$ are given in Definition 2.12 and $m_{H_{\sigma}}$ is the height of $H_{\sigma}$ as in Definition 2.4 for each $\sigma \in \Sigma$ where $H=\left(H_{\sigma}\right)_{\sigma \in \Sigma}$. For each subset $P \subset \mathbb{Z}$, define

$$
\mathcal{H}^{\mathrm{reg}}\left(\check{C}, a_{-}, a_{+}, P\right) \subset \mathcal{H}^{\mathbb{T}}\left(\check{C}, a_{-}, a_{+}\right)
$$

to be the subspace of time dependent Hamiltonians $H=\left(H_{t}\right)_{t \in \mathbb{T}}$ so that there exist neighborhoods $N_{-}, N_{+}$of $a_{-}$and $a_{+}$in $\operatorname{Sc}\left(Q_{-}\right)$and $\operatorname{Sc}\left(Q_{+}\right)$respectively so that for each $a_{ \pm}^{\prime} \in N_{ \pm}$, we have

- that every capped 1-periodic orbit in $\Gamma_{\tilde{C}, a_{-}^{\prime}, a_{+}^{\prime}}^{\mathbb{Z}}(H)$ is non-degenerate,

- there are no 1-periodic orbits contained in $[1+\epsilon / 8,1+\epsilon / 2] \times C$ and

- $\Gamma_{\check{C}, a_{-}^{\prime}, a_{+}^{\prime}}^{P}(H)=\Gamma_{\check{C}, a_{-}, a_{+}}^{P}(H)$.

Define $\mathcal{H}^{\mathrm{reg}}\left(\check{C}, a_{-}, a_{+}\right):=\mathcal{H}^{\mathrm{reg}}\left(\check{C}, a_{-}, a_{+}, \mathbb{Z}\right)$. 
We have that $\mathcal{H}^{\mathrm{reg}}\left(\check{C}, a_{-}, a_{+}\right)$is an open dense subset of $\mathcal{H}^{\mathbb{T}}\left(\check{C}, a_{-}, a_{+}\right)$by Lemma 8.3 in Appendix A. The height condition in Equation (2.16) is essential for this density property since any Hamiltonian in $\mathcal{H}^{\mathbb{T}}\left(\check{C}, a_{-}, a_{+}\right)$is constant outside $D \cup \check{C}$ if $\left(a_{-}, a_{+}\right)$is wide and therefore these constant orbits $\gamma$ must not satisfy Equation (2.14) because they are degenerate.

Definition 2.59. Let $\left(a_{-}, a_{+}\right) \in \operatorname{Sc}\left(Q_{-}\right) \times \operatorname{Sc}\left(Q_{+}\right)$be a $\check{C}$-action interval and let $H \in$ $\mathcal{H}^{\text {reg }}\left(\check{C}, a_{-}, a_{+},\{p\}\right)$ for some $p \in \mathbb{Z}$. Define $C F_{\check{C}, a_{ \pm}, \infty}^{p}(H)$ to be the free $\mathbb{K}$-module generated by capped 1-periodic orbits $\gamma$ of $H$ of index $p$ and satisfying $a_{ \pm} \leq\left.\mathcal{A}_{H, \check{C}}(\gamma)\right|_{Q_{ \pm}}$. Define

$$
C F_{\check{C}, a_{-}, a_{+}}^{p}(H):=C F_{\check{C}, a_{-}, \infty}^{p}(H) /\left(C F_{\check{C}, a_{-}, \infty}^{p}(H) \cap C F_{\check{C}, a_{+}, \infty}^{p}(H)\right) .
$$

Remark 2.60. The $\mathbb{K}$-module $C F_{\check{C}, a_{-}, a_{+}}^{p}(H)$ is naturally isomorphic to the free $\mathbb{K}$-module generated by $\Gamma_{\check{C}, a_{-}, a_{+}}^{p}(H):=\Gamma_{\check{C}, a_{-}, a_{+}}^{\{p\}}(H)$. From now on we will not distinguish between describing such a group as a quotient or a free module.

Definition 2.61. Let $H$ be a time dependent Hamiltonian on $M$. If $\gamma=(\widetilde{\gamma}, \check{\gamma})$ is a capped 1-periodic orbit of $H$ and $v \in H_{2}(M ; \mathbb{Z})$ is a homology class, then we define $\gamma \# v:=(\widetilde{\gamma} \# v, \check{\gamma})$ where $\widetilde{\gamma} \# v$ has the property that $(\widetilde{\gamma} \# v) \star \widetilde{\gamma}$ is homologous to $v$ where $\star$ is defined Equation $(2.2)$.

Above, we are 'connect summing' $v$ to the capping surface $\widetilde{\gamma}$.

Remark 2.62. Let $\left(a_{-}, a_{+}\right) \in\left(Q_{-}, Q_{+}\right)$be a $\check{C}$-action interval and let $H \in \mathcal{H}^{\mathrm{reg}}\left(\check{C}, a_{-}, a_{+},\{p\}\right)$ for some $p \in \mathbb{Z}$. Define $\check{Q}_{+}:=\iota_{D}\left(\pi_{D}\left(Q_{+}\right)\right)$. The submonoid

$$
S_{0}^{Q_{+}}:=\left\{x \in H_{2}(M ; \mathbb{Z}): 0 \preceq_{\check{Q}_{+}} x\right\} \subset H_{2}(M ; \mathbb{Z})
$$

acts on $C F_{\check{C}, a_{-}, a_{+}}^{p}(H)$ as follows: An element $v \in S_{0}^{Q_{+}}$represents the unique $\mathbb{K}$-linear map sending an element $\gamma \in \Gamma_{\check{C}, a_{-}, a_{+}}^{p}(H)$ to $[\gamma \#(-v)] \in C F_{\check{C}, a_{-}, a_{+}}^{p}(H)$. As a result, the monoid ring $\mathbb{K}\left[S_{0}^{Q+}\right]$ acts on $C F_{\check{C}, a_{-}, a_{+}}^{p}(H)$.

Also, since set of 1-periodic orbits of $H$ is a compact subset of the free loop space of $M$ with respect to the $C^{\infty}$ topology, there is an element $a \in S_{0}^{Q_{+}}$so that for each $v \in S_{0}^{Q+}$ satisfying $a \preceq_{\check{Q}_{+}} v$ and each $\alpha \in C F_{\check{C}, a_{-}, a_{+}}^{p}(H)$, we have $v \cdot \alpha=0$. This implies that the $\mathbb{K}\left[S_{0}^{Q_{+}}\right]$-action extends to an action of the positive $Q_{+}$-Novikov ring $\Lambda_{\mathbb{K}}^{Q_{+},{ }^{+}}$on $C F_{\check{C}, a_{-}, a_{+}}^{p}(H)$ by Equation (2.13). Hence from now on, we will think of $C F_{\check{C}, a_{-}, a_{+}}^{p}(H)$ as a $\Lambda_{\mathbb{K}}^{Q_{+},{ }^{+}}{ }_{\text {-module. }}$.

The $\Lambda_{\mathbb{K}}^{Q_{+},{ }^{+}}$-module $C F_{\tilde{C}, a_{-}, a_{+}}^{p}(H)$ will be the module underlying our chain complex for our Hamiltonian Floer group. We now need to explain what the differential on $C F_{\check{C}, a_{-}, a_{+}}^{p}(H)$ is.

Definition 2.63. Let $\left(a_{-}, a_{+}\right)$be a $\check{C}$-action interval and let $P:=\{p, p+1\}$ for some $p \in \mathbb{Z}$. Let $H \in \mathcal{H}^{\mathrm{reg}}\left(\check{C}, a_{-}, a_{+}, P\right)$ and let $J \in \mathcal{J}^{\mathbb{T}, \operatorname{reg}}(H, \check{C})$ where $\mathcal{J}^{\mathbb{T}, \mathrm{reg}}(H, \check{C})$ is given in Definition 2.23. We define the Floer differential

$$
\partial_{H, J}^{(p)}: C F_{\check{C}, a_{-}, a_{+}}^{p}(H) \longrightarrow C F_{\check{C}, a_{-}, a_{+}}^{p+1}(H)
$$

to be the unique $\mathbb{K}$-linear map satisfying

$$
\partial_{H, J}^{(p)}\left(\gamma_{+}\right)=\sum_{\gamma_{-} \in \Gamma_{\tilde{C}, a_{-}, a_{+}}^{p+1}(H)} \# \overline{\mathcal{M}}(H, J, \gamma) \gamma_{-}, \quad \forall \gamma_{+} \in \Gamma_{\check{C}, a_{-}, a_{+}}^{p}(H)
$$


where $\gamma$ is the pair $\left(\gamma_{-}, \gamma_{+}\right)$and where $\# \overline{\mathcal{M}}(H, J, \gamma)$ is the number of elements in the 0 dimensional manifold $\overline{\mathcal{M}}(H, J, \gamma)$ from Definition 2.23 counted with sign according to their orientation. We define $\partial_{H, J}:=\partial_{H, J}^{(p)}$ if it is clear which $p$ we are using.

The definition above uses the fact that $\overline{\mathcal{M}}(H, J, \gamma)$ is a compact oriented 0-dimensional manifold for all pairs of capped 1-periodic orbits $\gamma=\left(\gamma_{-}, \gamma_{+}\right) \in \Gamma_{\check{C}, a_{-}, a_{+}}^{p+1}(H) \times \Gamma_{\check{C}, a_{-}, a_{+}}^{p}(H)$ which follows from Propositions 2.24 and 10.5 and [Rit13, Section 17]. Note that one can always find $J$ as above since $\mathcal{J}^{\mathbb{T}, \text { reg }}\left(H, \check{C}\right.$ ) is a ubiquitous subset of $\mathcal{J}^{\mathbb{T}}(\check{C})$ (Definition 2.12) by Proposition 2.19. By analyzing 1-parameter families of solutions of the Floer equation for the cylinder as in Definition 2.20, one can show that $\partial_{H, J}^{2}=0$. This is done by a gluing theorem [AD14, Theorem 9.2.1] combined with the compactness result Proposition 10.5 and the orientation conventions [Rit13, Section 17]. Here we have replaced the compactness result [AD14, Theorem 9.1.7] with Proposition 10.5. Finally, note that $\partial_{H, J}$ is a $\Lambda_{\mathbb{K}}^{Q+,+}$-linear differential where $Q_{+}$is the domain of $a_{+}$. Hence we have the following definition.

Definition 2.64. Let $\left(a_{-}, a_{+}\right) \in \operatorname{Sc}\left(Q_{-}\right) \times \operatorname{Sc}\left(Q_{+}\right)$be a $\check{C}$-action interval and let $P:=$ $\{p-1, p, p+1\}$ for some $p \in \mathbb{Z}$. Let $H \in \mathcal{H}^{\mathrm{reg}}\left(\check{C}, a_{-}, a_{+}, P\right)$ and let $J \in \mathcal{J}^{\mathbb{T}, \operatorname{reg}}(H, \check{C})$. We define the Hamiltonian Floer cohomology group of $H$ to be the $\Lambda_{\mathbb{K}}^{Q_{+},+}{ }^{+}$-module

$$
H F_{\check{C}, a_{-}, a_{+}}^{p}(H):=\operatorname{ker}\left(\partial_{H, J}^{(p)}\right) / \operatorname{Im}\left(\partial_{H, J}^{(p-1)}\right) .
$$

This Hamiltonian Floer cohomology group does not depend on the choice $J$ by continuation map methods, which will be explained in the subsequent section. Here are the two main examples of Hamiltonian Floer cohomology groups that should be kept in mind.

Example 2.65. If $\check{C}$ is the empty contact cylinder and $Q_{-}=Q_{+}$is the cone spanned by $([\omega], 1,1)$ then we get the usual definition of Floer cohomology with the usual action functional (see Example 2.11).

Example 2.66. Let $q_{0}, \cdots, q_{k} \in H^{2}(M, D ; \mathbb{R})$ be classes representing $\check{C}$-compatible 2-forms whose images in $H^{2}(M ; \mathbb{R})$ are linearly independent. Suppose that $Q_{+}$is the polyhedral cone spanned by $\left(q_{0}, 1,1\right), \cdots,\left(q_{k}, 1,1\right)$ and $Q_{-}$is the polyhedral cone spanned by

$$
\left(q_{0}, 1,1\right), \cdots,\left(q_{k}, 1,1\right),\left(q_{0}, 0,1\right), \cdots,\left(q_{k}, 0,1\right) .
$$

Then for $\left(a_{-}, a_{+}\right) \in \operatorname{Sc}\left(Q_{-}\right) \times \operatorname{Sc}\left(Q_{+}\right)$and $H \in \mathcal{H}^{\mathrm{reg}}\left(\check{C}, a_{-}, a_{+}, \mathbb{Z}\right), H F_{\check{C}, a_{-}, a_{+}}^{*}(H)$ is the Hamiltonian Floer cohomology group generated by capped 1-periodic orbits $\gamma$ satisfying $\mathcal{A}_{\check{C}, H}(\gamma)\left(q_{i}, 1,1\right) \geq a_{-}\left(q_{i}, 1,1\right)$ and $\mathcal{A}_{\check{C}, H}(\gamma)\left(q_{i}, 0,1\right) \geq\left(q_{i}, 0,1\right)$ for all $i \in\{0, \cdots, k\}$ and $\mathcal{A}_{\check{C}, H}(\gamma)\left(q_{i}, 1,1\right) \leq a_{+}\left(q_{j}, 1,1\right)$ for some $j \in\{0, \cdots, k\}$ where $\mathcal{A}_{\check{C}, H}$ is defined in 2.10.

2.6. Continuation Maps. Again, throughout this subsection, we will fix a (possibly empty) contact cylinder $\check{C}=[1-\epsilon, 1+\epsilon] \times C \subset M$ with associated Liouville domain $D$ and cylindrical coordinate $r_{C}$. We will also fix a $\check{C}$-action interval $\left(a_{-}, a_{+}\right)$.

Definition 2.67. Let $H^{-}=\left(H_{t}^{-}\right)_{t \in \mathbb{T}}, H^{+}=\left(H_{t}^{+}\right)_{t \in \mathbb{T}} \in \mathcal{H}^{\mathbb{T}}\left(\check{C}, a_{-}, a_{+}\right)$have slopes $\left(\lambda_{H_{t}^{ \pm}}\right)_{t \in \mathbb{T}}$ and heights $\left(m_{H_{t}^{ \pm}}\right)_{t \in \mathbb{T}}$ at $\check{C}$ respectively. We write $H^{-}<_{\check{C}} H^{+}$if

(1) $H_{t}^{-}<H_{t}^{+}$,

(2) $\lambda_{H_{t}^{-}}<\lambda_{H_{t}^{+}}, m_{H_{t}^{-}}<m_{H_{t}^{+}}$

(3) $m_{t}^{+}-m_{t}^{-}<\left.\left(H_{t}^{+}-H_{t}^{-}\right)\right|_{M-(D \cup([1,1+\epsilon / 2] \times C)}$

for all $t \in \mathbb{T}$ (See Figure 6). 


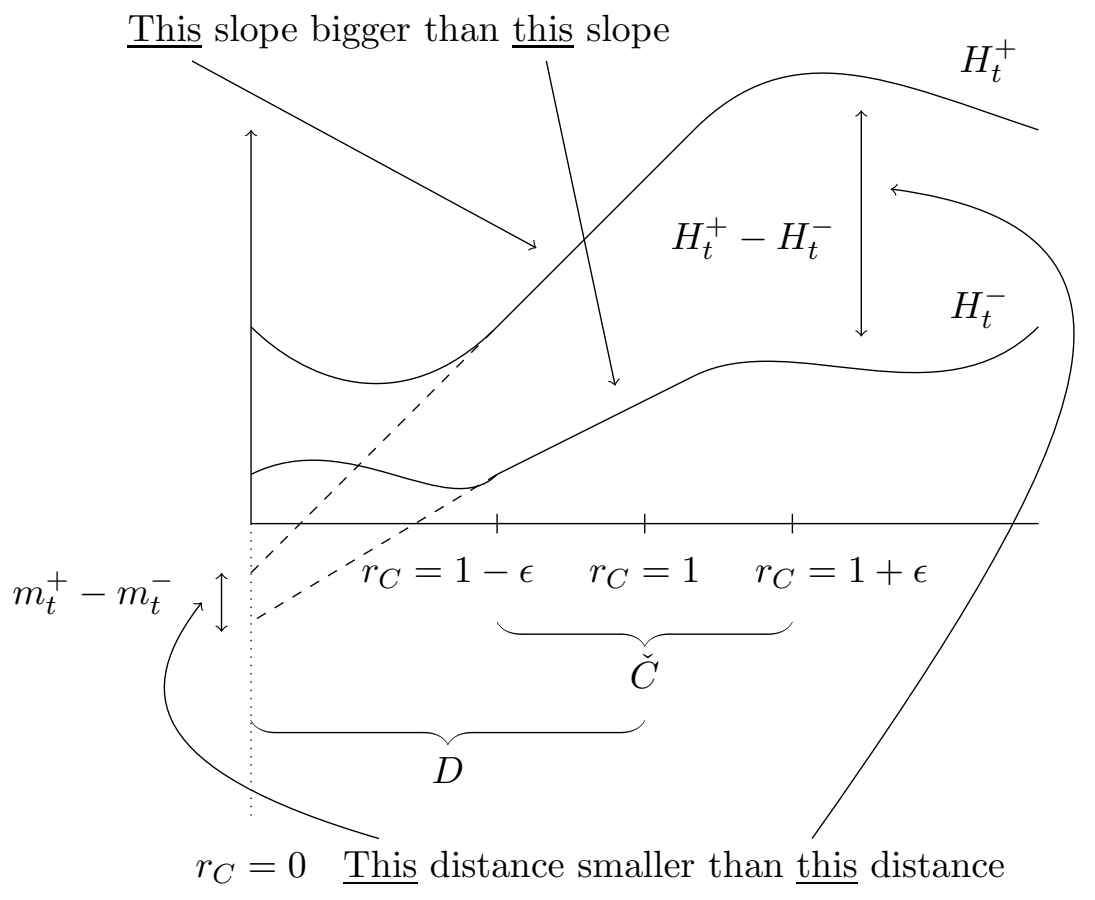

Figure 6. Picture of $H^{-}$and $H^{+}$satisfying $H^{-}<_{\check{C}} H^{+}$.

The following lemma below tells us that the relation $<_{\check{C}}$ has good properties.

Lemma 2.68. Let $H \in \mathcal{H}^{\mathbb{T}}\left(\check{C}, a_{-}, a_{+}\right)$. Then there is a sequence $\left(H_{i}\right)_{i \in \mathbb{N}}$ of elements in $\mathcal{H}^{\mathbb{T}}\left(\check{C}, a_{-}, a_{+}\right) C^{\infty}$ converging to $H$ so that for each $K<_{\check{C}} H$, there exists $i \in \mathbb{N}$ so that $K<_{\check{C}} H_{i}<_{\check{C}} H$.

Proof. Suppose $H=\left(H_{t}\right)_{t \in \mathbb{T}}$ and let $\lambda_{H_{t}}$ and $m_{H_{t}}$ be the slope and height of $H_{t}$ for each $t \in \mathbb{T}$. Let $f: \mathbb{R} \longrightarrow \mathbb{R}$ be a smooth function satisfying

$$
\left.f\right|_{(-\infty, 1]}=-2,\left.\quad f\right|_{[1+3 \epsilon / 4, \infty)}=-2-\epsilon, \quad f^{\prime} \leq 0
$$

and $f(x)=-1-x$ for all $x \in[1+\epsilon / 8,1+\epsilon / 2]$. Define

$$
K: M \longrightarrow \mathbb{R}, \quad K(x):= \begin{cases}-2 & \text { if } x \in D \\ f\left(r_{C}\right) & \text { if } x \in \check{C} \\ -2-\epsilon & \text { otherwise. }\end{cases}
$$

Then $H_{i, t}:=H_{t}+\frac{1}{i} K \in \mathcal{H}^{\mathbb{T}}(\check{C})$ has slope $\lambda_{H_{t}}-\frac{1}{i}$ and height $m_{H_{i, t}}:=m_{H_{t}}-\frac{1}{i}$. Also

$$
m_{H_{t}}-m_{H_{i, t}}<\frac{2+\epsilon / 2}{i} \leq\left.\left(H_{t}-H_{i, t}\right)\right|_{M-(D \cup([1,1+\epsilon / 2] \times C))} .
$$

Therefore $H_{i}:=\left(H_{i, t}\right)_{t \in \mathbb{T}}$ has the properties we want for all $i$ large enough.

Definition 2.69. Let $H^{-}, H^{+} \in \mathcal{H}^{\mathbb{T}}\left(\check{C}, a_{-}, a_{+}\right)$satisfy $H^{-}<_{\check{C}} H^{+}$. A smooth family of Hamiltonians $H^{-+}=\left(H_{s, t}^{-+}\right)_{(s, t) \in \mathbb{R} \times \mathbb{T}}\left(a_{-}, a_{+}\right)$-connects $H^{-}$with $H^{+}$if

- $H_{s}^{-+}:=\left(H_{s, t}^{-+}\right)_{t \in \mathbb{T}}$ is an element of $\mathcal{H}^{\mathbb{T}}\left(\check{C}, a_{-}, a_{+}\right)$for all $s \in \mathbb{R}$,

- $H_{s}^{-+}=H^{ \pm}$for all $s \in \mathbb{R}$ satisfying $\mp s \gg 1$ 
- and if $\left(\lambda_{H_{s, t}^{-+}}\right)_{(s, t) \in \mathbb{R} \times \mathbb{T}},\left(m_{H_{s, t}^{-+}}\right)_{(s, t) \in \mathbb{R} \times \mathbb{T}}$ are the corresponding slopes and heights of $\left(H_{s, t}^{-+}\right)_{(s, t) \in \mathbb{R} \times \mathbb{T}}$ along $\check{C}$ then

$$
\begin{gathered}
\frac{d}{d s} H_{s, t}^{-+}(x) \leq 0, \forall x \in M, \quad \frac{d}{d s} \lambda_{H_{s, t}^{-+}} \leq 0, \quad \frac{d}{d s} m_{H_{s, t}^{-+}} \leq 0, \\
\frac{d}{d s} H_{s, t}^{-+}(x) \leq \frac{d}{d s}\left(m_{H_{s, t}^{-+}}\right), \forall x \in M-(D \cup([1,1+\epsilon / 2] \times C))
\end{gathered}
$$

for all $t \in \mathbb{T}$. We will denote the space of such families of Hamiltonians by

$$
\mathcal{H}^{\mathbb{R} \times \mathbb{T}}\left(\check{C}, a_{-}, a_{+}, H^{-}, H^{+}\right) .
$$

Note that $\mathcal{H}^{\mathbb{R} \times \mathbb{T}}\left(\check{C}, a_{-}, a_{+}, H^{-}, H^{+}\right)$is contractible since it is a convex subset of the space $\mathcal{H}^{\mathbb{R} \times \mathbb{T}}\left(\check{C}, a_{-}, a_{+}\right)$with at least one element equal to:

$$
\left((1-\rho(s)) H_{t}^{+}+\rho(s) H_{t}^{-}\right)_{(s, t) \in \mathbb{R} \times \mathbb{T}}
$$

where $\rho: \mathbb{R} \longrightarrow \mathbb{R}$ is a smooth function satisfying $\rho^{\prime} \geq 0$ and where $\rho(s)$ is equal to 0 for $s \ll 0$ and 1 for $s \gg 0$.

Definition 2.70. If $H^{ \pm} \in \mathcal{H}^{\mathbb{T}}\left(\check{C}, a_{-}, a_{+}\right)$and $J^{ \pm} \in \mathcal{J}^{\mathbb{T}}(\check{C})$ (Definition 2.12) then we say $\left(H^{-+}, J^{-+}\right)\left(a_{-}, a_{+}\right)$-connects $\left(H^{-}, J^{-}\right)$with $\left(H^{+}, J^{+}\right)$if $H^{-+}\left(a_{-}, a_{+}\right)$-connects $H^{-}$with $H^{+}$and if $J^{-+} \in \mathcal{J}^{\mathbb{R} \times \mathbb{T}}\left(\left(J^{-}, J^{+}\right), \check{C}\right)$.

Definition 2.71. Let $H^{ \pm} \in \mathcal{H}^{\mathrm{reg}}\left(\check{C}, a_{-}, a_{+}, P\right)$ (Definition 2.16) where $P=\{p-1, p, p+1\}$ for some $p \in \mathbb{Z}$ and let $J^{ \pm} \in \mathcal{J}^{\mathbb{T} \text {,reg }}\left(H^{ \pm}, \check{C}\right)$ where $\mathcal{J}^{\mathbb{T} \text {,reg }}\left(H^{ \pm}, \check{C}\right)$ is given in Definition 2.23. Let $H^{-+} \in \mathcal{H}^{\mathbb{R} \times \mathbb{T}}\left(\check{C}, a_{-}, a_{+}, H^{-}, H^{+}\right)$and $J^{-+} \in \mathcal{J}^{\mathbb{R} \times \mathbb{T}}$,reg $\left(H^{-+},\left(J^{-}, J^{+}\right), \check{C}\right)$ (Proposition 2.19). The family $\left(H^{-+}, J^{-+}\right)$then defines for us a continuation map

$$
\Phi_{H^{-+}, J^{-+}}^{p}: H F_{\check{C}, a_{-}, a_{+}}^{p}\left(H^{-}\right) \longrightarrow H F_{\check{C}, a_{-}, a_{+}}^{p}\left(H^{+}\right)
$$

induced from the chain level continuation map

$$
\widetilde{\Phi}_{H^{-+}, J^{-+}}^{p}: C F_{\check{C}, a_{-}, a_{+}}^{p}\left(H^{-}\right) \longrightarrow C F_{\check{C}, a_{-}, a_{+}}^{p}\left(H^{+}\right)
$$

which is the unique $\mathbb{K}$-linear map satisfying

$$
\widetilde{\Phi}_{H^{-+}, J^{-+}}^{p}\left(\gamma_{+}\right):=\sum_{\gamma_{-} \in \Gamma_{\check{C}, a_{-}, a_{+}}^{p}\left(H^{+}\right)} \# \mathcal{M}\left(H^{-+}, J^{-+}, \gamma\right) \gamma_{-}, \quad \forall \gamma_{+} \in \Gamma_{\check{C}, a_{-}, a_{+}}^{p}\left(H^{-}\right)
$$

where $\gamma=\left(\gamma_{-}, \gamma_{+}\right)$and $\# \mathcal{N}\left(H^{-+}, J^{-+}, \gamma\right)$ is the number of elements in the 0-dimensional manifold $\mathcal{M}\left(H^{-+}, J^{-+}, \gamma\right)$ (Definition 2.14) counted with sign according to orientation. The set $\Gamma_{\check{C}, a_{-}, a_{+}}^{p}\left(H^{-}\right)$is defined in Definition 2.58.

The map $\Phi_{H^{-+}, J^{-+}}^{p}$ is well defined since the chain level map (2.18) induced by Equation (2.19) commutes with the differentials $\partial_{H^{ \pm}, J^{ \pm}}^{\left(p_{ \pm}\right)}, p_{-}=p-1, p_{+}=p$ by the gluing theorem [Sch95, Theorem 4.4.1] combined with the compactness result Proposition 10.5 and orientation conventions [Rit13, Section 17]. The same gluing theorem also tells us that the composition of two continuation maps is a continuation map. Finally, if we have other elements $K^{-+} \in \mathcal{H}^{\mathbb{R} \times \mathbb{T}}\left(\check{C}, a_{-}, a_{+}, H^{-}, H^{+}\right)$and $Y^{-+} \in \mathcal{J}^{\mathbb{R} \times \mathbb{T}, \mathrm{reg}}\left(K^{-+},\left(J^{-}, J^{+}\right), \check{C}\right)$ then $\Phi_{H^{-+}, J^{-+}}^{p}=\Phi_{K^{-+}, Y^{-+}}^{p}$. Such an equivalence of maps is given by a chain homotopy defined in a similar way to Equation (2.18), except that we count elements of $\mathcal{M}(F, L, \gamma)$ where $\Sigma$ • is the smooth family of Riemann surfaces $(\mathbb{R} \times \mathbb{T})_{\sigma \in[0,1]}$,

$$
F=\left(F_{\sigma, s, t}\right)_{(\sigma, s, t) \in[0,1] \times \mathbb{R} \times \mathbb{T}} \in \mathcal{H}^{\Sigma} \bullet\left(\left(H^{-}, H^{+}\right), \check{C}\right)
$$


satisfies $F_{0, s, t}=H_{s, t}^{-+}, F_{1, s, t}=K_{s, t}^{-+}$and

$$
\left(F_{\sigma, s, t}\right)_{(s, t) \in \mathbb{R} \times \mathbb{T}} \in \mathcal{H}^{\mathbb{R} \times \mathbb{T}}\left(\check{C}, a_{-}, a_{+}, H^{-}, H^{+}\right) \quad \forall \sigma \in[0,1]
$$

and where

$$
L \in \mathrm{J}^{\Sigma_{\bullet}, \mathrm{reg}}\left(F,\left(J^{-+}, Y^{-+}\right),\left(J^{-}, J^{+}\right), \check{C}\right)
$$

(See Proposition 2.21). This map is a chain homotopy by [AD14, Proposition 11.2.8] where we replace the compactness result [AD14, Theorem 11.3.7] by Proposition 10.5. As a result of the facts above we have the following definition.

Definition 2.72. Let $H^{ \pm} \in \mathcal{H}^{\text {reg }}\left(\check{C}, a_{-}, a_{+}, P\right)$ (Definition 2.58) satisfy $H^{-}<_{\check{C}} H^{+}$where $P=\{p-1, p, p+1\}$ for some $p \in \mathbb{Z}$. The continuation map

$$
\Phi_{H^{-}, H^{+}}^{p}: H F_{\check{C}, a_{-}, a_{+}}^{p}\left(H^{-}\right) \longrightarrow H F_{\check{C}, a_{-}, a_{+}}^{p}\left(H^{+}\right)
$$

is defined to be $\Phi_{H^{-+}, J^{-+}}^{p}$ for some choice of $\left(H^{-+}, J^{-+}\right)$as in Definition 2.69.

This is a $\Lambda_{\mathbb{K}}^{Q_{+},+}{ }^{-}$-module map. We also have the following important lemmas giving us sufficient conditions ensuring that a continuation map is an isomorphism.

Lemma 2.73. Let $H \in \mathcal{H}^{\mathrm{reg}}\left(\check{C}, a_{-}, a_{+}, P\right)$ where $P=\{p-1, p, p+1\}$ for some $p \in \mathbb{Z}$, and let $J \in \mathcal{J}^{\mathbb{T}}(\check{C})$. Then there is a convex neighborhood $U_{H} \subset \mathcal{H}^{\mathbb{T}}\left(\check{C}, a_{-}, a_{+}\right)$of $H$ (Definition 2.58) and a weakly contractible neighborhood $V_{J} \subset J^{\mathbb{T}}(\check{C})$ of $J$ so that for all $H^{ \pm} \in U_{H} \cap$ $\mathcal{H}^{\mathrm{reg}}\left(\check{C}, a_{-}, a_{+}, P\right), J^{ \pm} \in V_{J} \cap \mathcal{J}^{\mathbb{T}, \mathrm{reg}}\left(H^{ \pm}, \check{C}\right)$,

$$
\begin{aligned}
H^{-+} & =\left(H_{s, t}^{-+}\right)_{(s, t) \in \mathbb{R} \times \mathbb{T}} \in \mathcal{H}^{\mathbb{R} \times \mathbb{T}}\left(\check{C}, a_{-}, a_{+}, H^{-}, H^{+}\right), \\
J^{-+} & =\left(J_{s, t}^{-+}\right)_{(s, t) \in \mathbb{R} \times \mathbb{T}} \in \mathcal{J}^{\mathbb{R} \times \mathbb{T}, \operatorname{reg}}\left(H^{-+},\left(J^{-}, J^{+}\right), \check{C}\right)
\end{aligned}
$$

(Proposition 2.19) satisfying $\left(H_{s, t}^{-+}\right)_{t \in \mathbb{T}} \in U_{H}$ and $\left(V_{s, t}^{-+}\right)_{t \in \mathbb{T}} \in V_{J}$ for all $s \in \mathbb{R}$, the degree $p$ chain level continuation map $\widetilde{\Phi}_{H^{-+}, J^{-+}}^{p}$ from Equation (2.18) is an isomorphism.

Proof. The key idea of the proof of this lemma is to use a Gromov compactness result to show that the low energy solutions of the Floer equation defining the chain level continuation map induce an isomorphism. For each $K \in \mathcal{H}^{\mathrm{reg}}\left(\check{C}, a_{-}, a_{+}, P\right)$ and $t \in \mathbb{T}, \operatorname{let}^{\mathrm{ev}} t: \Gamma_{\check{C}, a_{-}, a_{+}}^{P}(K) \longrightarrow$ $M$ be the map sending a capped 1-periodic orbit $\gamma=(\widetilde{\gamma}, \check{\gamma})$ to $\widetilde{\gamma}(\check{\gamma}(t))$ and define $\Gamma_{K}:=$ $\operatorname{ev}_{0}\left(\Gamma_{\check{C}, a_{-}, a_{+}}^{P}(K)\right)$. Define $\Gamma:=\Gamma_{H}$. Since each element of $\Gamma_{\check{C}, a_{-}, a_{+}}^{P}(H)$ is non-degenerate, we have that $\Gamma$ is a finite subset of $M$. Therefore we can find open subsets $N_{\Gamma}^{\prime} \subset N_{\Gamma} \subset M$ of $\Gamma$ so that the inclusion maps $\Gamma \hookrightarrow N_{\Gamma}^{\prime}, N_{\Gamma}^{\prime} \hookrightarrow N_{\Gamma}$ are homotopy equivalences and so that the closure of $N_{\Gamma}^{\prime}$ is contained in $N_{\Gamma}$. Define $\mathcal{H}^{\mathbb{T}, \Gamma} \subset \mathcal{H}^{\text {reg }}\left(\check{C}, a_{-}, a_{+}, P\right)$ to be the subspace of Hamiltonians $K$ satisfying $\phi_{t}^{K}\left(\Gamma_{K}\right) \subset \phi_{t}^{H}\left(N_{\Gamma}^{\prime}\right)$ for all $t \in \mathbb{T}$ and so that the inclusion map $\Gamma_{K} \hookrightarrow N_{\Gamma}^{\prime}$ is a homotopy equivalence.

For each manifold $\Sigma$ and each subset $U_{0} \subset \mathcal{H}^{\mathbb{T}}(\check{C})$ and $V_{0} \subset \mathcal{J}^{\mathbb{R} \times \mathbb{T}}(\check{C})$, define

$$
\begin{gathered}
\left.\mathcal{H}^{\Sigma \times \mathbb{T}}(\check{C})\right|_{U_{0}}:=\left\{\left(K_{\sigma, t}\right)_{(\sigma, t) \in \Sigma \times \mathbb{T}} \in \mathcal{H}^{\Sigma \times \mathbb{T}}(\check{C}):\left(K_{\sigma, t}\right)_{t \in \mathbb{T}} \in U_{0} \forall \sigma \in \Sigma\right\}, \\
\left.\mathcal{J}^{\Sigma \times \mathbb{R} \times \mathbb{T}}(\check{C})\right|_{V_{0}}:=\left\{\left(K_{\sigma, s, t}\right)_{(\sigma, s, t) \in \Sigma \times \mathbb{R} \times \mathbb{T}} \in \mathcal{J}^{\Sigma \times \mathbb{R} \times \mathbb{T}}(\check{C}):\left(K_{\sigma, s, t}\right)_{(s, t) \in \mathbb{R} \times \mathbb{T}} \in V_{0} \forall \sigma \in \Sigma\right\}
\end{gathered}
$$

(See Definition 2.12). For each $U_{0} \subset \mathcal{H}^{\mathbb{T}}(\check{C})$, define $\mathcal{J}^{\mathbb{R} \times \mathbb{T}, \Gamma}\left(U_{0}\right) \subset \mathcal{J}^{\mathbb{R} \times \mathbb{T}}(\check{C})$ be the subspace of elements $Y=\left(Y_{s, t}\right)_{(s, t) \in \mathbb{R} \times \mathbb{T}}$ so that for each

- $K^{ \pm} \in \mathcal{H}^{\mathbb{T}, \Gamma} \cap U_{0}, \gamma^{ \pm} \in \Gamma_{\check{C}, a_{-}, a_{+}}^{P}\left(K^{ \pm}\right)$

- $\left.K \in \mathcal{H}^{\mathbb{R} \times \mathbb{T}}(\check{C})\right|_{\mathcal{H}^{\mathbb{T}}, \Gamma \cap U_{0}} \cap \mathcal{H}^{\mathbb{R} \times \mathbb{T}^{+}}\left(\left(K^{-}, K^{+}\right), \check{C}\right)$ and

- $u \in \mathcal{M}\left(K, Y,\left(\gamma_{-}, \gamma_{+}\right)\right)$(Definition 2.14) satisfying $u_{s, t} \in \phi_{t}^{H}\left(N_{\Gamma}\right)$ for all $(s, t) \in \mathbb{R} \times \mathbb{T}$, 
we have that $u(s, t) \in \phi_{t}^{H}\left(N_{\Gamma}^{\prime}\right)$ for all $(s, t) \in \mathbb{R} \times \mathbb{T}$. Morally, the definition above is used to find those almost complex structures $Y$ for which we can select an isolated low energy region of $\mathcal{M}\left(K, Y,\left(\gamma_{-}, \gamma_{+}\right)\right)$.

Since there are neighborhoods $N_{-}, N_{+}$of $a_{-}, a_{+}$so that $\Gamma_{\check{C}, a_{-}^{\prime}, a_{+}^{\prime}}^{P}(H)=\Gamma_{\check{C}, a_{-}, a_{+}}^{P}(H)$ for all $a_{ \pm}^{\prime} \in N_{ \pm}$, we have by a compactness argument (such as the one in [McL12b, Lemma 2.3]) that there exists a weakly contractible open neighborhood $U \subset \mathcal{H}^{\mathbb{T}}\left(\check{C}, a_{-}, a_{+}\right)$of $H$ satisfying $U \subset \mathcal{H}^{\mathbb{T}, \Gamma}$. Let

$$
\iota_{\mathrm{cpx}}: \mathcal{J}^{\mathbb{T}}(\check{C}) \longleftrightarrow \mathcal{J}^{\mathbb{T}}(\check{C}), \quad \iota_{\mathrm{cpx}}\left(\left(\check{J}_{t}\right)_{t \in \mathbb{T}}\right):=\left(\check{J}_{t}\right)_{(s, t) \in \mathbb{R} \times \mathbb{T}}
$$

be the natural inclusion map. By a Gromov compactness argument (such as the one in [McL12b, Lemma 2.3]) we have, after shrinking $U$ further, that there is a weakly contractible open subset $V \subset \mathcal{J}^{\mathbb{R} \times \mathbb{T}}(\check{C})$ of $\iota_{\mathrm{cpx}}(J)$ so that $V \subset \mathrm{J}^{\mathbb{R} \times \mathbb{T}, \Gamma}\left(U_{0}\right)$.

As a result, for each $K \in U$, we can define the Hamiltonian Floer cohomology group $H F^{p}\left(\left.K\right|_{N_{\Gamma}}\right)$ in the usual way except that we only consider capped 1-periodic orbits inside $\cap_{t \in \mathbb{T}} \operatorname{ev}_{t}^{-1}\left(\phi_{t}^{H}\left(N_{\Gamma}\right)\right)$, almost complex structures $\left(J_{t}\right)_{t \in \mathbb{T}}$ satisfying $\iota_{\mathrm{cpx}}\left(\left(J_{t}\right)_{t \in \mathbb{T}}\right) \in V$, and Floer trajectories $u: \mathbb{R} \times \mathbb{T} \longrightarrow M$ satisfying $u(s, t) \in \phi_{t}^{H}\left(N_{\Gamma}\right)$ for all $(s, t) \in \mathbb{R} \times \mathbb{T}$. For topological reasons we get that the differential vanishes and hence $H F^{p}\left(\left.K\right|_{N_{\Gamma}}\right)=C F^{p}\left(\left.K\right|_{N_{\Gamma}}\right)$ where $C F^{p}\left(\left.K\right|_{N_{\Gamma}}\right)$ is the degree $p$ part of the chain complex defining $H F^{p}\left(\left.K\right|_{N_{\Gamma}}\right)$.

Also by only considering families of Hamiltonians inside $\left.\mathcal{H}^{\mathbb{R} \times \mathbb{T}}(\check{C})\right|_{U},\left.\mathcal{H}^{[0,1] \times \mathbb{R} \times \mathbb{T}}(\check{C})\right|_{U}$, families of almost complex structures inside $V$ and $\left.j^{[0,1] \times \mathbb{R} \times \mathbb{T}}(\check{C})\right|_{V}$ and Floer trajectories $u: \mathbb{R} \times \mathbb{T} \longrightarrow M$ satisfying $u(s, t) \in \phi_{t}^{H}\left(N_{\Gamma}\right)$ for all $(s, t) \in \mathbb{R} \times \mathbb{T}$, one can show that the chain level continuation map $\widetilde{\Phi}_{K^{-+}, Y^{-+}}^{p}: C F^{p}\left(\left.K\right|_{N_{\Gamma}}\right) \longrightarrow C F^{p}\left(\left.\check{K}\right|_{N_{\Gamma}}\right)$ is an isomorphism for each $K, \check{K} \in U,\left.K^{-+} \in \mathcal{H}^{\mathbb{R} \times \mathbb{T}}(\check{C})\right|_{U} \cap \mathcal{H}^{\mathbb{R} \times \mathbb{T}}\left(\left(K^{-}, K^{+}\right), \check{C}\right)$ and $\left.Y^{-+} \in \mathcal{J}^{\mathbb{R} \times \mathbb{T}}(\check{C})\right|_{V} \cap$ $\mathcal{J}^{\mathbb{R} \times \mathbb{T}, \text { reg }}\left(K,\left(Y^{-}, Y^{+}\right), \check{C}\right)$ (see [AD14, Chapter 11]). The above arguments work because Gromov compactness [AD14, Theorem 9.1.7] still holds since there are no Floer trajectories $u$ as above satisfying $u(s, t) \in N_{\Gamma}-N_{\Gamma}^{\prime}$ for some $(s, t) \in \mathbb{R} \times \mathbb{T}$ (in other words we have selected appropriate clopen regions inside the moduli spaces of Floer cylinders needed to construct $\widetilde{\Phi}_{K^{-+}, Y^{-+}}^{p}$ and show it is an isomorphism).

Hence if we choose $U_{H} \subset U$ to be a convex neighborhood of $H$ and $V_{J} \subset V \cap \mathcal{J}^{\mathbb{T}}(\check{C})$ a weakly contractible neighborhood of $J$ then our lemma holds by an action filtration argument.

Very roughly, the following lemma says that if one has a family of Hamiltonians whose Floer chain complexes are all the same (after possibly pulling back by a diffeomorphism), then the corresponding continuation map is an isomorphism.

Lemma 2.74. Let $P=\left[p_{-}-1, p_{+}+1\right]$ for some $p_{ \pm} \in \mathbb{Z}$ and let $\left(a_{-}^{m}, a_{+}^{m}\right) \in \operatorname{Sc}\left(Q_{-}^{m}\right) \times \operatorname{Sc}\left(Q_{+}^{m}\right)$, $m \in I$ be a finite collection of $\check{C}$-action intervals. Suppose there exists $\left(H_{s, t}\right)_{(s, t) \in \mathbb{R} \times \mathbb{T}} \in$ $\mathcal{H}^{[0,1] \times \mathbb{T}}(\check{C})$ so that $H_{s, \bullet}:=\left(H_{s, t}\right)_{t \in \mathbb{T}} \in \cap_{m \in I} \mathcal{H}^{\mathrm{reg}}\left(\check{C}, a_{-}^{m}, a_{+}^{m}, P\right)$ (Definition 2.16) for all $s \in[0,1]$ and $H_{s, \bullet}<_{\check{C}} H_{\check{s}, \bullet}$ for each $s<\check{s}$. Let $J^{ \pm} \in \mathcal{J}^{\mathbb{T}, \text { reg }}\left(H^{ \pm}, \check{C}\right)$ where $H^{-}:=H_{0, \bullet}$ and $H^{+}:=H_{1, \bullet}$. Then there exists

$$
H^{-+} \in \cap_{m \in I} \mathcal{H}^{\mathbb{R} \times \mathbb{T}}\left(\check{C}, a_{-}^{m}, a_{+}^{m}, H^{-}, H^{+}\right), \quad J^{-+} \in \mathcal{J}^{\mathbb{R} \times \mathbb{T}, \mathrm{reg}}\left(H^{-+},\left(J^{-}, J^{+}\right), \check{C}\right)
$$

so that the chain level continuation map

$$
\widetilde{\Phi}_{H^{-+}, J^{-+}}^{p}: C F_{\check{C}, a_{-}^{m}, a_{+}^{m}}^{p}\left(H^{-}\right) \longrightarrow C F_{\check{C}, a_{-}^{m}, a_{+}^{m}}^{p}\left(H^{+}\right)
$$

is an isomorphism for each $p_{-} \leq p \leq p_{+}$and each $m \in I$. In particular the continuation map $\Phi_{H^{-}, H^{+}}^{p}: H F_{\check{C}, a_{-}^{m}, a_{+}^{m}}^{p}\left(H^{-}\right) \longrightarrow H F_{\check{C}, a_{-}^{m}, a_{+}^{m}}^{p}\left(H^{+}\right)$is an isomorphism for each such $p$ and $m$. 
Proof. Here the key idea of the proof is to chop up the homotopy $\left(H_{s, t}\right)_{(s, t) \in[0,1] \times \mathbb{T}}$ into many small homotopies and then apply Lemma 2.73 above. Let $J \in \mathcal{J}^{\mathbb{T}}(\check{C})$. Let $U_{H_{s}, \bullet}^{m} \subset$ $\mathcal{H}^{\mathbb{T}}\left(\check{C}, a_{-}^{m}, a_{+}^{m}\right)$ and $V_{H_{s} \bullet}^{m} \subset \mathcal{J}^{\mathbb{T}}\left(\check{C}, a_{-}^{m}, a_{+}^{m}\right)$ be open neighborhoods of $H_{s, \bullet}$ and $J$ respectively so that the conclusion of Lemma 2.73 holds with $H$ and $\left(a_{-}, a_{+}\right)$replaced by $H_{s, \bullet}$ and $\left(a_{-}^{m}, a_{+}^{m}\right)$ respectively for each $m \in I$. Let $U_{H_{s}, \bullet} \subset \cap_{m \in I} U_{H_{s}, \bullet}^{m}$ be a convex neighborhood of $H_{s, \bullet}$ and $V_{H_{s}, \bullet} \subset \cap_{m \in I} V_{H_{s}, \bullet}^{m}$ a weakly contractible open neighborhood of $J$ for each $s \in[0,1]$. Since $[0,1]$ is compact, we can choose $s_{0}=0<s_{1}<s_{2} \cdots<s_{k}=1$ so that $H_{s_{j+1}, \bullet} \in U_{H_{s_{j}}}$ • or $H_{s_{j}, \bullet} \in U_{H_{s_{j+1}}, \bullet}$ for all $0 \leq j \leq k-1$. Since $H^{-}<_{\check{C}} H^{+}$we have, by applying Lemma 2.68 inductively, that there exists $\check{H}_{j} \in U_{H_{s_{j}}, \bullet} \cap \cap_{i \in I} \mathcal{H}^{\mathrm{reg}}\left(\check{C}, a_{-}^{m}, a_{+}^{m}, P\right), 0 \leq j \leq k$ satisfying $\check{H}_{j}<_{\check{C}} \check{H}_{j+1}$ and $\check{H}_{j}, \check{H}_{j+1} \in U_{H_{s_{j}} \text { • }}$ or $\check{H}_{j}, \check{H}_{j+1} \in U_{H_{s_{j+1}}, \bullet}$ for all $0 \leq j \leq k-1$ and satisfying $\check{H}_{0}=H_{0, \bullet}$ and $\check{H}_{k}=H_{1, \bullet}$. Now choose $\widetilde{J} \in \cap_{j=1}^{k}\left(V_{H_{s_{j}}, \bullet} \cap \mathcal{J}^{\mathbb{T}, \text { reg }}\left(\check{H}_{j}, \check{C}\right)\right)$ and choose

$$
\begin{gathered}
H^{-+, j}=\left(H_{s, t}^{-+, j}\right)_{(s, t) \in \mathbb{R} \times \mathbb{T}} \in \cap_{m \in I} \mathcal{H}^{\mathbb{R} \times \mathbb{T}}\left(\check{C}, a_{-}^{m}, a_{+}^{m}, \check{H}_{j}, \check{H}_{j+1}\right), \\
J^{-+, j}=\left(J_{s, t}^{-+, j}\right)_{(s, t) \in \mathbb{R} \times \mathbb{T}} \in J^{\mathbb{R} \times \mathbb{T}, \operatorname{reg}}\left(H^{-+},(\widetilde{J}, \widetilde{J}), \check{C}\right)
\end{gathered}
$$

satisfying $\left(H_{s, t}^{-+, j}\right)_{t \in \mathbb{T}} \in U_{H_{s_{j^{\prime}}}, \bullet}$ and $\left(V_{s, t}^{-+, j}\right)_{t \in \mathbb{T}} \in V_{H_{s^{\prime}}, \bullet}$ for all $s \in \mathbb{R}$, for some $j^{\prime} \in\{j, j+1\}$ (independent of $s$ ) and for all $0 \leq j \leq k-1$. Then by Lemma 2.73, we have that the degree $p$ chain level continuation map $\widetilde{\Phi}_{H^{-+, j}, J^{-+, j}}^{p}$ from Equation (2.18) is an isomorphism for all $0 \leq j \leq k-1$. By a repeated gluing argument [AD14, Theorem 11.1.16] applied to $\left(H^{-+, j}, J^{-+, j}\right)$ for all $0 \leq j \leq k-1$, there exists $\left(H^{-+}, J^{-+}\right)$as in Equation (2.22) so that so that the chain level continuation map

$$
\widetilde{\Phi}_{H^{-+}, J^{-+}}^{p}: C F_{\check{C}, a_{-}^{m}, a_{+}^{m}}^{p}\left(H^{-}\right) \longrightarrow C F_{\check{C}, a_{-}^{m}, a_{+}^{m}}^{p}\left(H^{+}\right)
$$

is an isomorphism for each $p_{-} \leq p \leq p_{+}$and $m \in I$.

2.7. Action Maps. Throughout this subsection, we will fix a (possibly empty) contact cylin$\operatorname{der} \check{C}=[1-\epsilon, 1+\epsilon] \times C \subset M$ with associated Liouville domain $D$.

Definition 2.75. Let $\left(a_{-}^{j}, a_{+}^{j}\right) \in \operatorname{Sc}\left(Q_{-}^{j}\right) \times \operatorname{Sc}\left(Q_{+}^{j}\right)$ be a $\check{C}$-action interval for $j=0,1$. We say that $\left(a_{-}^{1}, a_{+}^{1}\right)$ is smaller than $\left(a_{-}^{0}, a_{+}^{0}\right)$ if $Q_{ \pm}^{1} \subset Q_{ \pm}^{0}$ and if $a_{ \pm}^{1} \leq\left. a_{ \pm}^{0}\right|_{Q_{ \pm}^{1}}$.

Remark 2.76. If $\left(a_{-}^{1}, a_{+}^{1}\right)$ is smaller than $\left(a_{-}^{0}, a_{+}^{0}\right)$ then we have induced morphisms of Novikov rings $\Lambda_{\mathbb{K}}^{Q_{+}^{0}} \longrightarrow \Lambda_{\mathbb{K}}^{Q_{+}^{1}}$ and $\Lambda_{\mathbb{K}}^{Q_{+}^{0},+} \longrightarrow \Lambda_{\mathbb{K}}^{Q_{+}^{1},+}$. In particular, any $\Lambda_{\mathbb{K}}^{Q_{+}^{1}}$ (resp. $\Lambda_{\mathbb{K}}^{Q_{+}^{1},{ }^{+}}$) module is naturally a $\Lambda_{\mathbb{K}}^{Q_{+}^{0}}$ (resp. $\Lambda_{\mathbb{K}}^{Q_{+}^{0},+}$ ) module.

Definition 2.77. Let $P=\{p-1, p, p+1\}$ for some $p \in \mathbb{Z}$. Let $\left(a_{-}^{j}, a_{+}^{j}\right)$ be a $\check{C}$-action interval for $j=0,1$ so that $\left(a_{-}^{1}, a_{+}^{1}\right)$ is smaller than $\left(a_{-}^{0}, a_{+}^{0}\right)$. Let $H \in \cap_{j=0,1} \mathcal{H}^{\mathrm{reg}}\left(\check{C}, a_{-}^{j}, a_{+}^{j}, P\right)$. Then we have a natural chain map

$$
C F_{\tilde{C}, a_{-}^{0}, a_{+}^{0}}^{q}(H) \stackrel{\alpha}{\longrightarrow} C F_{\tilde{C}, a_{-}^{0}, \infty}^{q}(H) /\left(C F_{\tilde{C}, a_{-}^{0}, \infty}^{q}(H) \cap C F_{\tilde{C}, a_{+}^{1}, \infty}^{q}(H)\right) \stackrel{\beta}{\longrightarrow} C F_{\tilde{C}, a_{-}^{1}, a_{+}^{1}}^{q}(H)
$$

for each $q \in P$ called a chain level action map where $\alpha$ is the natural quotient map and $\beta$ is the natural inclusion map of $\mathbb{K}$-modules. The induced map on homology

$$
H F_{\check{C}, a_{-}^{0}, a_{+}^{0}}^{p}(H) \longrightarrow H F_{\check{C}, a_{-}^{1}, a_{+}^{1}}^{p}(H)
$$

is called an action map. 
Remark 2.78. Action maps are morphisms of $\Lambda_{\mathbb{K}}^{Q_{+}^{0},{ }^{+}}$-modules where $Q_{+}^{0}$ is the domain of $a_{+}^{0}$ (see Remark 2.76). The composition of two action maps is an action map. Action maps commute with continuation maps by Lemma 10.3 .

We also have the following important lemma giving us a sufficient condition for an action map to be an isomorphism.

Lemma 2.79. Let $Q_{j},\left(a_{-}^{j}, a_{+}^{j}\right), j=0,1, P, p, H$ be as in Definition 2.7\%. Suppose that $\Gamma_{\check{C}, a_{-}^{0}, a_{+}^{0}}^{P}(H)=\Gamma_{\check{C}, a_{-}^{1}, a_{+}^{1}}^{P}(H)$ (Definition 2.58). Then the action map

$$
H F_{\check{C}, a_{-}^{0}, a_{+}^{0}}^{p}(H) \longrightarrow H F_{\check{C}, a_{-}^{1}, a_{+}^{1}}^{p}(H)
$$

is an isomorphism.

Proof. This follows from the fact that the chain maps $\alpha$ and $\beta$ from Equation (2.23) are isomorphisms in degree $p$.

2.8. Invariance Under Time Reparameterization. Throughout this subsection, $\check{C}$ is a contact cylinder whose associated Liouville domain is $D$. We will also fix a $\breve{C}$-interval domain $\left(Q_{-}, Q_{+}\right)$.

Definition 2.80. Let $F: \mathbb{T} \longrightarrow \mathbb{T}$ be a smooth non-decreasing map. Let $H=\left(H_{t}\right)_{t \in \mathbb{T}}$ be a smooth Hamiltonian. We define $H^{F}=\left(H_{t}^{F}\right)_{t \in \mathbb{T}}$ by $H_{t}^{F}:=F^{\prime}(t) H_{F(t)}$ for each $t \in \mathbb{T}$.

Proposition 2.81. Let $F: \mathbb{T} \longrightarrow \mathbb{T}$ be a smooth non-decreasing map which is homotopic to the identity map. Let $\left(a_{-}, a_{+}\right) \in \mathrm{Sc}\left(Q_{-}\right) \times \operatorname{Sc}\left(Q_{+}\right)$be a $\check{C}$-action interval and $P:=$ $\{p-1, p, p+1\}$ for some $p \in \mathbb{Z}$. Then for each $H \in \mathcal{H}^{\mathrm{reg}}\left(\breve{C}, a_{-}, a_{+}, P\right)$ there is an isomorphism

$$
H F_{\breve{C}, a_{-}, a_{+}}^{*}(H) \cong H F_{\breve{C}, a_{-}, a_{+}}^{*}\left(H^{F}\right)
$$

which commutes with continuation maps and action maps.

Definition 2.82. The isomorphism (2.25) will be called a reparameterization isomorphism.

Proof of Proposition 2.81. The correspondence sending a loop $\gamma: \mathbb{T} \longrightarrow M$ to the loop $\gamma \circ F$ induces a bijection between 1-periodic orbits of $H$ and $H^{F}$ respectively. Since $F$ is isotopic through non-decreasing maps to the identity map, we have that this correspondence lifts in a natural way to capped 1-periodic orbits. Such a bijection induces an isomorphism of modules $C F_{\check{C}, a_{-}, a_{+}}^{p}(H) \cong C F_{\check{C}, a_{-}, a_{+}}^{p}\left(H^{F}\right)$ for each $p \in P$. Since $F$ is homotopic to the identity map, there is a unique function $G: \mathbb{T} \longrightarrow \mathbb{R}$ satisfying $G(0)=0$ and $G^{\prime}(t)=F^{\prime}(t)-1$. Let $J=\left(J_{t}\right)_{t \in \mathbb{T}} \in \mathcal{J}^{\operatorname{reg}}(H, \check{C})$ and define $J^{F}:=\left(J_{F(t)}\right)_{t \in \mathbb{T}}$. Then there is a bijection between $(H, J)$-Floer cylinders $u: \mathbb{R} \times \mathbb{T} \longrightarrow M$ and $\left(H^{F}, J^{F}\right)$-Floer cylinders

$$
u^{F}: \mathbb{R} \times \mathbb{T} \longrightarrow M, \quad u^{F}(s, t):=u(s+G(t), F(t)) .
$$

Also $J^{F} \in \operatorname{Jreg}^{\mathrm{re}}\left(H^{F}, \check{C}\right)$ since the corresponding Fredholm operators linearizing the Floer equation are canonically identified via a similar correspondence. Putting everything together we get our isomorphism (2.25). By considering a similar correspondence for Floer trajectories defining continuation maps as in Definition 2.72, we see that such an isomorphism commutes with continuation maps. Finally, since these isomorphisms preserve action we get that they also respect action maps. 
2.9. The pair of pants product. Throughout this subsection we will fix a (possibly empty) contact cylinder $\check{C}=[1-\epsilon, 1+\epsilon] \times C \subset M$ with associated Liouville domain $D$.

Definition 2.83. Let $\left(a_{-}^{j}, a_{+}^{j}\right) \in \operatorname{Sc}\left(Q_{-}^{j}, Q_{+}^{j}\right)$ be a $\check{C}$-action interval for $j=0,1,2$. We say that $\left(a_{-}^{2}, a_{+}^{2}\right)$ is smaller than $\left(a_{-}^{j}, a_{+}^{j}\right)_{j=0,1}$ if $Q_{ \pm}^{2} \subset Q_{ \pm}^{j}$ for $j=0,1$ and

$$
a_{-}^{2} \leq\left. a_{-}^{0}\right|_{Q_{-}^{2}}+\left.a_{-}^{1}\right|_{Q_{-}^{2}}, \quad a_{+}^{2} \leq \min \left(\left.a_{-}^{0}\right|_{Q_{+}^{2}}+\left.a_{+}^{1}\right|_{Q_{+}^{2}},\left.a_{-}^{1}\right|_{Q_{+}^{2}}+\left.a_{+}^{0}\right|_{Q_{+}^{2}}\right) .
$$

Most of the time, it will be the case that

$$
Q_{0}=Q_{1}=Q_{2}, \quad a_{-}^{2}=a_{-}^{0}+a_{-}^{1} \text { and } a_{+}^{2}=\min \left(a_{-}^{0}+a_{+}^{1}, a_{+}^{0}+a_{-}^{1}\right) .
$$

Definition 2.84. For any time dependent Hamiltonian $H=\left(H_{t}\right)_{t \in \mathbb{T}}$ and any $k \in \mathbb{N}$, define $(k) H:=\left((k) H_{t}\right)_{t \in \mathbb{T}}$ where $(k) H_{t}:=k H_{k t}$ for all $t \in \mathbb{T}$.

Definition 2.85. Let $\left(a_{-}^{j}, a_{+}^{j}\right)$ be a $\check{C}$-action interval for $j=0,1,2$ so that $\left(a_{-}^{2}, a_{+}^{2}\right)$ is smaller than $\left(a_{-}^{j}, a_{+}^{j}\right)_{j=0,1}$. Define $\kappa_{0}=\kappa_{1}:=1$ and $\kappa_{2}:=2$. Let $H^{j}=\left(H_{t}^{j}\right)_{t \in \mathbb{T}}, j=0,1,2$ be Hamiltonians so that $\left(\kappa_{j}\right) H^{j} \in \cap_{k=0}^{2} \mathcal{H}^{\mathrm{reg}}\left(\check{C}, a_{-}^{k}, a_{+}^{k}\right)$ for $j=0,1,2$. Suppose that for each triple $\left(\gamma^{j}\right)_{j=0,1,2} \in \prod_{j=0,1,2} \Gamma_{\check{C}, a_{-}^{j}, a_{+}^{j}}^{p_{j}}\left(\kappa_{j} H^{j}\right)$ of capped 1-periodic orbits where $p_{0}, p_{1}, p_{2} \in \mathbb{Z}$, we have that the associated 1-periodic orbits of at least two of them have distinct images in $M$.

If $H^{j}<_{\breve{C}} H^{2}$ for $j=0,1$, we have a natural pair of pants product map

$$
\Phi_{H^{0}, H^{1}, H^{2}}: H F_{\tilde{C}, a_{-}^{0}, a_{+}^{0}}^{p_{0}}\left(H^{0}\right) \otimes_{\mathbb{K}} H F_{\check{C}, a_{-}^{1}, a_{+}^{1}}^{p_{1}}\left(H^{1}\right) \longrightarrow H F_{\check{C}, a_{-}^{2}, a_{+}^{2}}^{p_{0}+p_{1}}\left((2) H^{2}\right)
$$

induced from a chain level map

$$
\widetilde{\Phi}_{H, J}: C F_{\check{C}, a_{-}^{0}, a_{+}^{0}}^{p_{0}}\left(H^{0}\right) \otimes_{\mathbb{K}} C F_{\check{C}, a_{-}^{1}, a_{+}^{1}}^{p_{1}}\left(H^{1}\right) \longrightarrow C F_{\check{C}, a_{-}^{2}, a_{+}^{2}}^{p_{0}+p_{1}}\left((2) H^{2}\right)
$$

which is the unique $\mathbb{K}$-linear map satisfying

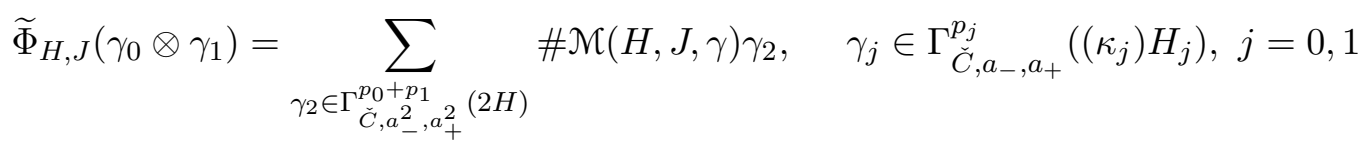

for some fixed $(H, J)$ where

- $H \in \mathcal{H}^{\Sigma}\left(\left(H^{0}, H^{1}, H^{2}\right), \check{C}\right)$ where $\Sigma$ is the pair of pants as in Example 2.25,

- $J \in \cap_{k=0}^{2} \mathcal{J}^{\Sigma, \text { reg }}\left(H,\left(J^{0}, J^{1}, J^{2}\right)\right) \cap \mathcal{J}^{\Sigma}(\check{C})$ where $J^{j} \in \mathcal{J}^{\mathbb{T}, \operatorname{reg}}\left(\kappa_{j} H^{j}, \check{C}\right)$ for all $j=0,1,2$ and

- $\gamma=\left(\gamma^{j}\right)_{j=0,1,2}$.

Remark 2.86. The condition $H^{j}<_{\check{C}} H^{2}$ for $j=0,1$ ensures that the Hamiltonian $H$ exists by considering the branched cover $\varpi$ from Example 2.25. The pair of pants product is well defined since the chain level map (2.26) commutes with the differentials by a gluing argument [Sch95, Theorem 4.4.1] combined with the compactness result Proposition 10.5 and orientation conventions [Rit13, Section 17]. Since the space of families of Hamiltonians $H$ as above is contractible and since the space $\mathcal{J}^{\Sigma}\left(\left(J^{0}, J^{1}, J^{2}\right), \check{C}\right)$ is contractible, we have (by looking at the moduli spaces in Definition 2.20 and Proposition 2.21) that $\Phi_{H^{0}, H^{1}, H^{2}}$ does not depend on $\Sigma, H$ or $J$ or the choice of $\Sigma$-compatible 1-form. Such a fact follows from [Sch95, Section 5.2] where we only consider families of Hamiltonians and almost complex structures from Definition 2.20 and where the compactness result [Sch95, Proposition 5.2.3] is replaced with Proposition 10.5. 
If $Q_{+}^{0}=Q_{+}^{1}$ then $\Phi_{H^{0}, H^{1}, H^{2}}$ descends to a $\Lambda^{Q_{+}^{0},{ }^{+}}{ }_{-}$bilinear map. A gluing argument [Sch95, Proposition 5.4.4] together with the use of the moduli spaces in Definition 2.20 and Proposition 2.21 tells us that this product commutes with continuation maps and is associative. Since $\Phi_{H^{0}, H^{1}, H^{2}}$ only depends on the oriented diffeomorphism type of $\Sigma$, we get that it is commutative when $a_{ \pm}^{0}=a_{ \pm}^{1}$ (see [Sch95, Section 5.5.1.3]). The pair of pants product also commutes with action maps since this product respects the filtrations on the Floer chain complexes by Lemma 10.3. If the constant Hamiltonian $\min \left(H^{0}\right)$ satisfies $\min \left(H^{0}\right)<_{\check{C}} \check{H}^{0}$ for some $C^{\infty}$ small perturbation $\check{H}^{0}$ of $H$ and if $a_{-}^{0}\left([\widetilde{\omega}], \lambda^{-}, \lambda^{+}\right)<\lambda^{-} \min \left(H^{0}\right)$ for all $\left([\widetilde{\omega}], \lambda^{-}, \lambda^{+}\right) \in Q_{0}-0$ and $p_{0}=0$ then this product has a left unit $\mathbf{1} \in H F_{\tilde{C}, a_{-}^{0}, a_{+}^{0}}^{p_{0}}\left(H^{0}\right)$ by [Sch95, Section 5.5.1.3] together with the gluing and compactness results stated above. By left unit we mean that for each $H^{3} \in \mathcal{H}^{\mathrm{reg}}\left(\check{C}, a_{-}^{2}, a_{+}^{2}\right)$ satisfying $2 H^{2}, H^{2}<_{\check{C}} H^{3}, \Phi_{2 H^{2}, H^{3}}^{p_{1}}\left(\Phi_{H^{0}, H^{1}, H^{2}}(\mathbf{1} \otimes x)\right)$ is the image of $x$ under the natural composition

$$
H F_{\check{C}, a_{-}^{1}, a_{+}^{1}}^{p_{1}}\left(H^{1}\right) \longrightarrow H F_{\check{C}, a_{-}^{1}, a_{+}^{1}}^{p_{1}}\left(H^{3}\right) \longrightarrow H F_{\check{C}, a_{-}^{2}, a_{+}^{2}}^{p_{0}+p_{1}}\left(H^{3}\right)
$$

where the first map is a continuation map and the second map is an action map for each $x \in H F_{\check{C}, a_{-}^{1}, a_{+}^{1}}^{p_{1}}\left(H^{1}\right)$. If $H^{1}$ satisfies similar conditions then we have a right unit. Left (resp. right) units get sent to left (resp. right) units under continuation and action maps.

\section{Floer Cohomology for Lower Semi-Continuous Hamiltonians}

In this section we introduce Hamiltonian Floer cohomology for certain lower semi-continuous Hamiltonians. This will be the direct limit of Floer cohomology groups of smooth Hamiltonians strictly smaller than such a lower semi-continuous Hamiltonian. These modules will basically satisfy all the same properties as the Floer groups defined in Section 2. Such ideas have been considered in [Gro15].

3.1. Main Definitions. Throughout this subsection we will fix a contact cylinder $\check{C}=$ $[1-\epsilon, 1+\epsilon] \times C$ with associated Liouville domain $D$ and cylindrical coordinate $r_{C}$. We will also fix a $\check{C}$-action interval $\left(a_{-}, a_{+}\right)$.

Definition 3.1. Recall that a function $f: X \longrightarrow \mathbb{R} \cup\{\infty\}$ from a metric space $X$ is lower semi-continuous if for all $x_{0} \in X$, $\liminf _{x \rightarrow x_{0}} f(x) \geq f\left(x_{0}\right)$. A lower semi-continuous Hamiltonian is a family of functions $\left(H_{t}\right)_{t \in \mathbb{T}}$ from $M$ to $\mathbb{R} \cup\{\infty\}$ so that the function

$$
\widehat{H}: \mathbb{T} \times M \longrightarrow \mathbb{R} \cup\{\infty\}, \quad \widehat{H}(t, x):=H_{t}(x)
$$

is lower semi-continuous. Such a Hamiltonian is continuous if $\widehat{H}$ is continuous.

A lower semi-continuous Hamiltonian $H=\left(H_{t}\right)_{t \in \mathbb{T}}$ is weakly $\check{C}$-compatible if the restriction $\left.H_{t}\right|_{[1+\epsilon / 8,1+\epsilon / 2] \times C}$ is either equal to $\infty$ or $\lambda_{H_{t}} r_{C}+m_{H_{t}}$ for some constants $\lambda_{H_{t}}, m_{H_{t}}$ for each $t \in \mathbb{T}$. We also require that the maps $t \rightarrow \lambda_{H_{t}}$ and $t \rightarrow m_{H_{t}}$ from $\mathbb{T}$ to $\mathbb{R}$ are lower semicontinuous where $\lambda_{H_{t}}:=0$ and $m_{H_{t}}:=\infty$ if $\left.H_{t}\right|_{[1+\epsilon / 8,1+\epsilon / 2] \times C}=\infty$. The slope of $H_{t}$ along $\check{C}$ is defined to be $\lambda_{H_{t}}$ and the height of $H_{t}$ along $\check{C}$ is defined to be $m_{H_{t}}$ for all $t \in \mathbb{T}$. Also the slope and height of $H_{t}$ is defined to be 0 if $\check{C}$ is the empty contact cylinder. We say that $H$ is $\check{C}$-compatible if it is weakly $\check{C}$-compatible and if $\left.H_{t}\right|_{M-(D \cup \check{C})}$ is constant (possibly equal to $\infty$ ) for each $t \in \mathbb{T}$. We define $\mathcal{H}^{\mathbb{T}, 1 . s .}(\check{C})\left(\operatorname{resp} . \overline{\mathcal{H}}^{\mathbb{T}, 1 . s .}(\check{C})\right)$ to be the set of weakly $\check{C}$-compatible (resp. $\check{C}$-compatible) lower semi-continuous Hamiltonians $H$. Define

$$
\mathcal{H}^{\mathbb{T}, \text { l.s. }}\left(\check{C}, a_{-}, a_{+}\right):=
$$




$$
\begin{cases}\mathcal{H}^{\mathbb{T}, 1 . s .}(\check{C}) & \text { if }\left(a_{-}, a_{+}\right) \text {is small } \\ \left\{H \in \overline{\mathcal{H}}^{\mathbb{T}, 1 . s .}(\check{C}): m_{H_{t}}>\operatorname{height}\left(a_{-}, a_{+}\right) \forall t \in \mathbb{T}\right\} & \text { otherwise }\end{cases}
$$

where height $\left(a_{-}, a_{+}\right)$is defined as in Equation (2.15) and where $\left(m_{H_{t}}\right)_{t \in \mathbb{T}}$ are the heights of $H=\left(H_{t}\right)_{t \in \mathbb{T}}$. For $H^{-}, H^{+} \in \mathcal{H}^{\mathbb{T}, 1 . s .}(\check{C})$, we say $H^{-} \leq_{\check{C}}^{\text {l.s. }} H^{+}$if

(1) $H_{t}^{-} \leq H_{t}^{+}$,

(2) $\lambda_{H_{t}^{-}} \leq \lambda_{H_{t}^{+}}, m_{H_{t}^{-}} \leq m_{H_{t}^{+}}$,

(3) $m_{H_{t}^{+}}-m_{H_{t}^{-}} \leq\left.\left(H_{t}^{+}-H_{t}^{-}\right)\right|_{M-(D \cup([1,1+\epsilon / 2] \times C)}$ (where we define $\infty-\infty$ to be 0$)$

for all $t \in \mathbb{T}$ where $\left(\lambda_{H_{t}^{ \pm}}\right)_{t \in \mathbb{T}},\left(m_{H_{t}^{ \pm}}\right)_{t \in \mathbb{T}}$ are the slopes and heights of $H^{ \pm}$.

For any $P \subset \mathbb{Z}$ and any $H \in \mathcal{H}^{\mathbb{T}, \text { l.s. }}\left(\check{C}, a_{-}, a_{+}\right)$, define $\mathcal{H}^{\text {reg }}\left(<_{\check{C}} H, a_{-}, a_{+}, P\right)$ to be the subspace of smooth Hamiltonians $\check{H}=\left(\check{H}_{t}\right)_{t \in \mathbb{T}} \in \mathcal{H}^{\mathrm{reg}}\left(\check{C}, a_{-}, a_{+}, P\right)$ (Definition 2.16) satisfying

- $\check{H}_{t}<H_{t}$,

- $\lambda_{\check{H}_{t}}<\lambda_{H_{t}}, m_{\check{H}_{t}}<m_{H_{t}}$ and

- $m_{H_{t}^{+}}-m_{H_{t}^{-}}<\left.\left(H_{t}^{+}-H_{t}^{-}\right)\right|_{M-(D \cup([1,1+\epsilon / 2] \times C)}$ for all $t \in \mathbb{T}$.

Define $\leq_{\check{C}}$ to be the relation on $\mathcal{H}^{\text {reg }}\left(<_{\check{C}} H, a_{-}, a_{+}, P\right)$ where $H^{-} \leq_{\check{C}} H^{+}$if $H^{-}=H^{+}$or $H^{-}<_{\check{C}} H^{+}$. Also define $\mathcal{H}^{\text {reg }}\left(<_{\check{C}} H, a_{-}, a_{+}\right):=\mathcal{H}^{\text {reg }}\left(<_{\check{C}} H, a_{-}, a_{+}, \mathbb{Z}\right)$.

Lemma 3.2. For any $P \subset \mathbb{Z}$ and any $H \in \mathcal{H}^{\mathbb{T}, 1 . \mathrm{s} .}\left(\check{C}, a_{-}, a_{+}, P\right)$, we have that $\left(\mathcal{H}^{\text {reg }}\left(<_{\check{C}}\right.\right.$ $\left.\left.H, a_{-}, a_{+}, P\right), \leq_{\check{C}}\right)$ is a non-empty directed set.

Proof. It is clear that $\leq_{\check{C}}$ is reflexive and transitive. We now need to show that ever pair of elements has an upper bound. Now suppose $H^{j}=\left(H_{t}^{j}\right)_{t \in \mathbb{T}} \in \mathcal{H}^{\mathrm{reg}}\left(<_{\check{C}} H, a_{-}, a_{+}, P\right)$ for $j=0,1$. Let $\left(\lambda_{H_{t}}\right)_{t \in \mathbb{T}}$, and $\left(m_{H_{t}}\right)_{t \in \mathbb{T}}\left(\operatorname{resp} . \quad\left(\lambda_{H_{t}^{j}}\right)_{t \in \mathbb{T}}\right.$ and $\left.\left(m_{H_{t}^{j}}\right)_{t \in \mathbb{T}}\right)$ be the slopes and heights of $H=\left(H_{t}\right)_{t \in \mathbb{T}}$ (resp. $H^{j}=\left(H_{t}^{j}\right)_{t \in \mathbb{T}}$ for $j=0,1$ ). Since $\lambda_{H_{t}}$ and $m_{H_{t}}$ varies in a lower semi-continuous way with respect to $t \in \mathbb{T}$, we can find smooth families of constants $\left(\lambda_{t}\right)_{t \in \mathbb{T}}, \quad\left(m_{t}\right)_{t \in \mathbb{T}}$ so that $\lambda_{H_{t}^{j}}<\lambda_{t}<\lambda_{H_{t}}$ and $m_{H_{t}^{j}}<m_{t}<m_{H_{t}}$ for all $t \in \mathbb{T}$. Also since $H$ is lower semi-continuous, we can find a smooth Hamiltonian $\check{H}=\left(\check{H}_{t}\right)_{t \in \mathbb{T}}$ so that $H_{t}^{j}<\check{H}_{t}<H_{t}$ for all $t \in \mathbb{T}$ and also so that $\left.\check{H}_{t}\right|_{M-(\check{C} \cup D)}$ is constant for each $t \in \mathbb{T}$ if $\left(a_{-}, a_{+}\right)$ is not small. We can also choose $\check{H}$ so that $\left.\left(H_{t}-\check{H}_{t}-\left(m_{H_{t}}-m_{t}\right)\right)\right|_{M-(D \cup([1,1+\epsilon / 2] \times C))}>0$ and $\left.\left(\check{H}_{t}-H_{t}^{j}-\left(m_{t}-m_{H_{t}^{j}}\right)\right)\right|_{M-(D \cup([1,1+\epsilon / 2] \times C))}>0$ for all $j=0,1$ and $t \in \mathbb{T}$. Then by using a bump function on $\check{C}$ to interpolate between $\check{H}_{t}$ and $\lambda_{t} r_{C}+m_{t}$ in the region $((1,1+\epsilon / 16) \cup(1+\epsilon / 2,1+3 \epsilon / 4)) \times C$, we can assume that $\left.\check{H}_{t}\right|_{[1+\epsilon / 8,1+\epsilon / 2] \times C}=\lambda_{t} r_{C}+m_{t}$ for all $t \in \mathbb{T}$ as well. Hence $\check{H} \in \mathcal{H}^{\mathbb{T}}\left(\check{C}, a_{-}, a_{+}\right)$and $H^{j}<_{\check{C}} \check{H} \leq_{\check{C}}^{\text {l.s. }} H$. By Lemma 2.68 combined with the fact that $\mathcal{H}^{\mathbb{T}}\left(\check{C}, a_{-}, a_{+}\right)$is a ubiquitous subset of $\mathcal{H}^{\mathbb{T}}(\check{C})$ by Lemma 8.3, we can find $H^{2} \in \mathcal{H}^{\mathrm{reg}}\left(\check{C}, a_{-}, a_{+}\right)$satisfying $H^{j}<_{\check{C}} H^{2}<_{\check{C}} \check{H}$ for $j=0,1$. Hence $H^{2} \in \mathcal{H}^{\mathrm{reg}}\left(<_{\check{C}} H, a_{-}, a_{+}\right)$and $H^{j}<_{\check{C}} H^{2}$ for $j=0,1$. A similar construction also shows that $\mathcal{H}^{\mathrm{reg}}\left(<_{\check{C}} H, a_{-}, a_{+}\right)$is non-empty. This completes our lemma.

Definition 3.3. Let $H \in \mathcal{H}^{\mathbb{T}, 1 . s .}\left(\check{C}, a_{-}, a_{+}\right)$. By Lemma 3.2, we can define

$$
H F_{\check{C}, a_{-}, a_{+}}^{p}(H):=\lim _{\breve{H} \in \mathcal{F}^{\mathrm{reg}}\left(<_{\check{C}} H, a_{-}, a_{+}, P\right)} H F_{\check{C}, a_{-}, a_{+}}^{*}(\check{H})
$$

for any $P$ containing $\{p-1, p, p+1\}$ where the direct limit is taken with respect to the directed system $\left(\mathcal{H}^{\mathrm{reg}}\left(<_{\check{C}} H, a_{-}, a_{+}, P\right), \leq_{\check{C}}\right)$ whose morphisms are continuation maps. 
If the lower semi-continuous Hamiltonian $H$ from Definition 3.3 is in fact smooth and an element of $\mathcal{H}^{\mathrm{reg}}\left(Q, a_{-}, a_{+}, P\right)$ then it would be good to check that Definitions 3.3 and 2.64 agree. We will do this now.

Lemma 3.4. Let $p \in \mathbb{Z}$ and let $H \in \mathcal{H}^{\mathrm{reg}}\left(\check{C}, a_{-}, a_{+}, P\right)$ where $P=\{p-1, p, p+1\}$. Let $G_{1}$ be equal to $H F_{\check{C}, a_{-}, a_{+}}^{p}(H)$ from Definition 2.64 and let $G_{2}$ be equal to $H F_{\check{C}, a_{-}, a_{+}}^{p}(H)$ from Definition 3.3. Then the natural map $G_{2} \longrightarrow G_{1}$ induced by continuation maps is an isomorphism.

As a result, we have consistent notation and we do not need to distinguish between Definition 2.64 and Definition 3.3.

Proof of Lemma 3.4. For any $\check{H} \in \mathcal{H}^{\mathrm{reg}}\left(<_{\check{C}} H, a_{-}, a_{+}, P\right)$, we let $H F_{\check{C}, a_{-}, a_{+}}^{p}(\check{H})$ be as in Definition 2.64. By Lemma 2.40, it is sufficient for us to show that there is a cofinal family $\Xi$ inside $\left(\mathcal{H}^{\mathrm{reg}}\left(<_{\check{C}} H, a_{-}, a_{+}, P\right), \leq_{\check{C}}\right)$ so that the natural map $H F_{\check{C}, a_{-}, a_{+}}^{p}(\check{H}) \longrightarrow G_{1}$ is an isomorphism for each element $\check{H}$ of this cofinal family. Let $U_{H} \subset \mathcal{H}^{\mathbb{T}}\left(\check{C}, a_{-}, a_{+}\right)$be the neighborhood of $H$ satisfying the properties of Lemma 2.73. Define $\Xi:=U_{H} \cap \mathcal{H}^{\mathrm{reg}}\left(<_{\check{C}} H, a_{-}, a_{+}, P\right)$. Then $\Xi$ is a cofinal family by Lemma 2.68 and the natural map $H F_{\check{C}, a_{-}, a_{+}}^{*}(\check{H}) \longrightarrow G_{1}$ is an isomorphism for all $\check{H} \in \Xi$ by Lemma 2.73. This completes the lemma.

3.2. Continuation Maps. Throughout this subsection, fix a contact cylinder $\check{C}$ together with a $\check{C}$-action interval $\left(a_{-}, a_{+}\right)$.

Definition 3.5. Let $p \in \mathbb{Z}$ and let $P \subset \mathbb{Z}$ satisfy $\{p-1, p, p+1\} \subset P$. Let $H^{-}, H^{+} \in$ $\mathcal{H}^{\text {l.s. }}\left(\check{C}, a_{-}, a_{+}\right)$satisfy $H^{-} \leq_{\check{C}}^{\text {l.s. }} H^{+}$. Then we have a natural inclusion of directed systems

$$
\mathcal{H}^{\mathrm{reg}}\left(<_{\check{C}} H^{-}, a_{-}, a_{+}, P\right) \subset \mathcal{H}^{\mathrm{reg}}\left(<_{\check{C}} H^{+}, a_{-}, a_{+}, P\right)
$$

and this induces a morphism

$$
\Phi_{H^{-}, H^{+}}^{p}: H F_{\check{C}, a_{-}, a_{+}}^{p}\left(H^{-}\right) \longrightarrow H F_{\check{C}, a_{-}, a_{+}}^{p}\left(H^{+}\right)
$$

called a continuation map.

If $H^{-}, H^{+}$from Definition 3.5 above are elements of $\mathcal{H}^{\mathrm{reg}}\left(\check{C}, a_{-}, a_{+}, P\right)$ then the continuation map from this definition is equal to the continuation map from Definition 2.72 by Lemma 3.4. The composition of two continuation maps is a continuation map.

The following Lemma is a generalization of a slightly weaker version of Lemma 2.74 to all smooth Hamiltonians (not just ones in $\mathcal{H}^{\mathrm{reg}}\left(\check{C}, a_{-}, a_{+}\right)$).

Lemma 3.6. Let $H=\left(H_{s, t}\right)_{(s, t) \in[0,1] \times \mathbb{T}}$ be a smooth family of autonomous smooth Hamiltonians and define $H_{s, \bullet}:=\left(H_{s, t}\right)_{t \in \mathbb{T}}$ for all $s \in[0,1]$. Fix $p \in \mathbb{Z}$. Suppose

(1) $H_{s, \bullet} \in \mathcal{H}^{\mathbb{T}, 1 . s .}\left(\check{C}, a_{-}, a_{+}\right)$for each $s \in[0,1]$ and $H_{s_{1}, \bullet} \leq_{\check{C} . s .}^{\text {l.s. }} H_{s_{2}}, \bullet$ for all $s_{1} \leq s_{2}$,

(2) there are neighborhoods $N_{-}, N_{+}$of $a_{-}, a_{+}$in $\operatorname{Sc}\left(Q_{-}\right), \operatorname{Sc}\left(Q_{+}\right)$respectively so that

$$
\Gamma_{\check{C}, a_{-}, a_{+}}^{P}\left(H_{s, \bullet}\right)=\Gamma_{\check{C}, a_{-}^{\prime}, a_{+}^{\prime}}^{P}\left(H_{s, \bullet}\right)
$$

(Definition 2.58) for all $a_{ \pm}^{\prime} \in N_{ \pm}, s \in[0,1]$ where $P=[p-1-n, p+1+n]$

(3) and that there are no 1 -periodic orbits of $H_{s, \bullet}$ contained in $[1+\epsilon / 8,1+\epsilon / 2] \times C$ for all $s \in[0,1]$. 
Then the continuation map

$$
\Phi_{H_{0, \bullet}, H_{1, \bullet}}^{p}: H F_{\check{C}, a_{-}, a_{+}}^{p}\left(H_{0, \bullet}\right) \longrightarrow H F_{\check{C}, a_{-}, a_{+}}^{p}\left(H_{1, \bullet}\right)
$$

in degree $p$ is an isomorphism.

Proof. This lemma will be proven by showing that for each $s \in[0,1]$ there is a constant $\epsilon_{s}>0$ so that the continuation map

$$
H F_{\check{C}, a_{-}, a_{+}}^{p}\left(H_{s_{0}}\right) \longrightarrow H F_{\check{C}, a_{-}, a_{+}}^{p}\left(H_{s_{1}}\right)
$$

is an isomorphism for all $s_{0}, s_{1} \in[0,1] \cap\left(s-\epsilon_{s}, s+\epsilon_{s}\right)$ satisfying $s_{0} \leq s_{1}$. So from now on, fix $s \in[0,1]$.

By (3) there are constants $\delta_{s}, \zeta>0$ so that for each $s^{\prime} \in\left[s-\delta_{s}, s+\delta_{s}\right]$ that every 1-periodic orbit of $H_{s^{\prime}, \bullet}$ has image not intersecting $N:=[1+\epsilon / 8-\zeta, 1+\epsilon / 2+\zeta] \times C$. By Lemma 2.68, there exists a cofinal family $\left(K_{i, s^{\prime}}\right)_{i \in \mathbb{N}}$ in $\mathcal{H}^{\text {reg }}\left(<_{\check{C}} H_{s, \bullet}, a_{-}, a_{+}, P\right)$ which $C^{\infty}$ converges to $H_{s^{\prime}, \bullet}$ for each $s^{\prime} \in[0,1]$.

Let $F: M \longrightarrow \mathbb{R}$ be a $\check{C}$-compatible autonomous Hamiltonian so that $F$ is locally constant outside $N$ and so that $0<_{\check{C}} F$. Since $F$ is locally constant outside $N$, there is a constant $0<\eta_{s}<\delta_{s}$ so that for each $s^{\prime} \in\left(s-\eta_{s}, s+\eta_{s}\right)$ and $\tau \in\left[-\eta_{s}, \eta_{s}\right]$ there is a constant $N_{s^{\prime}}>0$ so that $K_{i, s^{\prime}}+\tau F \in \mathcal{H}^{\mathbb{T}, \text { reg }}\left(\check{C}, a_{-}, a_{+}, P\right)$ for all $i \geq N_{s^{\prime}}$ by assumptions (2) and (3). Hence by Lemma 2.74, the continuation maps $H F_{\check{C}, a_{-}, a_{+}}^{p}\left(K_{i, s^{\prime}}+\tau^{-} F\right) \longrightarrow H F_{\check{C}, a_{-}, a_{+}}^{p}\left(K_{i, s^{\prime}}+\tau^{+} F\right)$ are isomorphisms for all $s^{\prime} \in\left(s-\eta_{s}, s+\eta_{s}\right), i \geq N_{s^{\prime}}$ and $\tau^{-}, \tau^{+} \in\left[-\eta_{s}, \eta_{s}\right]$ satisfying $\tau^{-}<\tau^{+}$ and hence the continuation maps $H F_{\tilde{C}, a_{-}, a_{+}}^{p}\left(H_{s^{\prime}, \bullet}+\tau^{-} F\right) \longrightarrow H F_{\tilde{C}, a_{-}, a_{+}}^{p}\left(H_{s^{\prime}, \bullet}+\tau^{+} F\right)$ are isomorphisms for all $s^{\prime} \in\left(s-\eta_{s}, s+\eta_{s}\right)$ and $\tau^{-}, \tau^{+} \in\left[-\eta_{s}, \eta_{s}\right]$ satisfying $\tau^{-} \leq \tau^{+}$.

Choose $0<\epsilon_{s}<\eta_{s}$ small enough so that

$$
H_{s_{1}, \bullet}-\eta_{s} F \leq \leq_{\tilde{C}}^{\text {l.s. }} H_{s_{0}, \bullet} \leq_{\tilde{C}}^{\text {l.s. }} H_{s_{1}, \bullet} \leq_{\tilde{C}}^{\text {l.s. }} H_{s_{0}, \bullet}+\eta_{s} F
$$

for all $s_{0}, s_{1} \in\left(s-\epsilon_{s}, s+\epsilon_{s}\right)$ satisfying $s_{0} \leq s_{1}$. By the discussion above combined with the fact that the composition of any two continuation maps is a continuation map, we get that the composition of any two maps in

$$
H F_{\check{C}, a_{-}, a_{+}}^{p}\left(H_{s_{1}, \bullet-} \eta_{s} F\right) \rightarrow H F_{\check{C}, a_{-}, a_{+}}^{p}\left(H_{s_{0}, \bullet}\right) \stackrel{\alpha}{\longrightarrow} H F_{\check{C}, a_{-}, a_{+}}^{p}\left(H_{s_{1}, \bullet}\right) \rightarrow H F_{\check{C}, a_{-}, a_{+}}^{p}\left(H_{s_{0}, \bullet}+\eta_{s} F\right)
$$

is an isomorphism. Hence $\alpha$ is an isomorphism for all $s_{0}, s_{1} \in\left(s-\epsilon_{s}, s+\epsilon_{s}\right)$ satisfying $s_{0} \leq s_{1}$.

3.3. Action Maps. We can also define action maps for lower semi-continuous Hamiltonians in a similar way to Subsection 2.7. Throughout this section we will fix a contact cylinder $\breve{C}=[1-\epsilon, 1+\epsilon] \times C \subset M$ with associated Liouville domain $D$.

Definition 3.7. Let $P=\{p-1, p, p+1\}$ for some $p \in \mathbb{Z}$. Let $\left(a_{-}^{j}, a_{+}^{j}\right)$ be a $\check{C}$-action interval for $j=0,1$ so that $\left(a_{-}^{1}, a_{+}^{1}\right)$ is smaller than $\left(a_{-}^{0}, a_{+}^{0}\right)$. Let $H \in \cap_{j=0,1} \mathcal{H}^{\mathbb{T}, \text { l.s. }}\left(\check{C}, a_{-}^{j}, a_{+}^{j}\right)$ (Definition 3.1) and let $\Xi:=\cap_{j=0,1} \mathcal{H}^{\mathrm{reg}}\left(<_{\check{C}} H, a_{-}^{j}, a_{+}^{j}\right)$ be the directed set with relation $\leq_{\check{C}}$. Since

$$
H F_{\check{C}, a_{-}^{j}, a_{+}^{j}}^{p}(H)=\lim _{\breve{H} \in \Xi} H F_{\check{C}, a_{-}^{j}, a_{+}^{j}}^{p}(\check{H}), \quad j=0,1
$$

and since action maps commute with continuation maps, we get that the action maps $H F_{\check{C}, a_{-}^{0}, a_{+}^{0}}^{*}(\check{H}) \longrightarrow H F_{\check{C}, a_{-}^{1}, a_{+}^{1}}^{*}(\check{H})$ for each $\check{H} \in \Xi$ from Definition 2.77 induce a map

$$
H F_{\check{C}, a_{-}^{0}, a_{+}^{0}}^{p}(H) \longrightarrow H F_{\check{C}, a_{-}^{1}, a_{+}^{1}}^{p}(H)
$$


which we also call an action map.

Again action maps commute with continuation maps. If the Hamiltonian $H$ is an element of $\cap_{j=0,1} \mathcal{H}^{\mathrm{reg}}\left(\check{C}, a_{-}^{j}, a_{+}^{j},\{p-1, p, p+1\}\right)$, then the action map from Definition 2.77 is equal to the action map from Definition 3.7 by Lemma 3.4. We also have the following analogue of Lemma 2.79.

Lemma 3.8. Let $P=[p-1-n, p+1+n]$ for some $p \in \mathbb{Z}$. Let $\left(a_{-}^{j}, a_{+}^{j}\right)$ be a $\check{C}$-action interval for $j=0,1$ so that $\left(a_{-}^{1}, a_{+}^{1}\right)$ is smaller than $\left(a_{-}^{0}, a_{+}^{0}\right)$. Let $H \in \cap_{j=0,1} \mathcal{H}^{\mathbb{T}, 1 . s .}\left(\check{C}, a_{-}^{j}, a_{+}^{j}\right)$ be a smooth Hamiltonian. Suppose that $\Gamma_{\check{C}, a_{-}^{0}, a_{+}^{0}}^{P}(H)=\Gamma_{\check{C}, a_{-}^{1}, a_{+}^{1}}^{P}(H)$. Then the action map

$$
H F_{\check{C}, a_{-}^{0}, a_{+}^{0}}^{p}(H) \longrightarrow H F_{\check{C}, a_{-}^{1}, a_{+}^{1}}^{p}(H)
$$

is an isomorphism.

Proof. By Lemma 2.68 there exists a cofinal family $\left(H^{i}\right)_{i \in \mathbb{N}}$ in $\cap_{j=0,1} \mathcal{H}^{\mathrm{reg}}\left(<_{\check{C}} H, a_{-}^{j}, a_{+}^{j}\right)$ so that $H^{i} C^{\infty}$ converges to $H$. Then for all $i$ sufficiently large, we have by (CZ7) from Definition 2.16 that $\Gamma_{\tilde{C}, a_{-}^{0}, a_{+}^{0}}^{\{p-1, p, p+1\}}\left(H^{i}\right)=\Gamma_{\tilde{C}, a_{-}^{1}, a_{+}^{1}}^{\{p-1\}}\left(H^{i}\right)$. Hence by Lemma 2.79, the action morphism

$$
H F_{\check{C}, a_{-}^{0}, a_{+}^{0}}^{p}\left(H^{i}\right) \longrightarrow H F_{\check{C}, a_{-}^{1}, a_{+}^{1}}^{p}\left(H^{i}\right)
$$

is an isomorphism for all $i$ sufficiently large. Therefore since action maps commute with continuation maps and since Equation (3.3) holds, we get our result.

3.4. Invariance Under Time Reparameterization. Throughout this subsection, $\check{C}$ is a contact cylinder whose associated Liouville domain is $D$. We will also fix a $\breve{C}$-interval domain $\left(Q_{-}, Q_{+}\right)$.

Definition 3.9. Let $F: \mathbb{T} \longrightarrow \mathbb{T}$ be a smooth non-decreasing map. Let $H=\left(H_{t}\right)_{t \in \mathbb{T}}$ be a lower semi-continuous Hamiltonian. We define $H^{F}=\left(H_{t}^{F}\right)_{t \in \mathbb{T}}$ by $H_{t}^{F}:=F^{\prime}(t) H_{F(t)}$.

Proposition 3.10. Let $F: \mathbb{T} \longrightarrow \mathbb{T}$ be a smooth non-decreasing map which is homotopic to the identity map. Let $\left(a_{-}, a_{+}\right) \in \operatorname{Sc}\left(Q_{-}\right) \times \operatorname{Sc}\left(Q_{+}\right)$be a $\check{C}$-action interval. Then for each $H \in \mathcal{H}^{\mathbb{T}, \text { l.s. }}\left(\check{C}, a_{-}, a_{+}\right)$there is an isomorphism of $\Lambda^{Q_{+},{ }_{-}-\text {modules }}$

$$
H F_{\breve{C}, a_{-}, a_{+}}^{*}(H) \cong H F_{\breve{C}, a_{-}, a_{+}}^{*}\left(H^{F}\right)
$$

which commutes with continuation maps and action maps.

Definition 3.11. The isomorphism (3.4) will be called a reparameterization isomorphism.

Proof of Proposition 3.10. Since

$$
H F_{\check{C}, a_{-}, a_{+}}^{p}\left(H^{F}\right)=\lim _{\check{H} \in \mathcal{H}^{\operatorname{reg}}\left(<_{\check{C}} H, a_{-}, a_{+}, \mathbb{Z}\right)} H F_{\check{C}, a_{-}, a_{+}}^{*}\left(\check{H}^{F}\right)
$$

by Proposition 2.81 and since the reparameterization isomorphisms

$$
H F_{\check{C}, a_{-}, a_{+}}^{*}(\check{H}) \cong H F_{\check{C}, a_{-}, a_{+}}^{*}\left(\check{H}^{F}\right)
$$

from Definition 2.82 commute with continuation maps, we get our isomorphism (3.4). 
3.5. Pair of Pants Product. Throughout this section we will fix a contact cylinder $\check{C}=$ $[1-\epsilon, 1+\epsilon] \times C \subset M$ with associated Liouville domain $D$.

Definition 3.12. Let $\left(a_{-}^{j}, a_{+}^{j}\right)$ be a $\check{C}$-action interval for $j=0,1,2$ so that $\left(a_{-}^{2}, a_{+}^{2}\right)$ is smaller than $\left(a_{-}^{j}, a_{+}^{j}\right)_{j=0,1}$ as in Definition 2.83. Define $\kappa_{0}=\kappa_{1}:=1$ and $\kappa_{2}:=2$. Let $H^{j}=\left(H_{t}^{j}\right)_{t \in \mathbb{T}}$ be a lower semi-continuous Hamiltonian so that $\left(\kappa_{j}\right) H^{j} \in \mathcal{H}^{\mathbb{T}, 1 . s .}\left(\check{C}, a_{-}^{j}, a_{+}^{j}\right)$ for $j=0,1,2$ where $\left(\kappa_{j}\right) H^{j}$ is defined as in Definition 2.84 and suppose $H^{j} \leq \leq_{\tilde{C}}^{\mathrm{ss} .} H^{2}$ for $j=0,1$.

Choose a cofinal sequence $\left(\left(\kappa_{j}\right) \check{H}^{i, j}\right)_{i \in \mathbb{N}}$ in $\cap_{k=0}^{2} \mathcal{H}^{\mathrm{reg}}\left(<_{\check{C}}, a_{-}^{k}, a_{+}^{k},\left(\kappa_{j}\right) H^{j}\right)$ (Definition 3.1) for each $j=0,1,2$ so that $\check{H}^{i, j}<_{\check{C}} \check{H}^{2, j}$ for $j=0,1$ and so that for each triple of capped 1-periodic orbits $\left(\gamma^{j}\right)_{j=0,1,2} \in \prod_{j=0,1,2} \Gamma_{\breve{C}, a_{-}^{j}, a_{+}^{j}}^{p_{j}}\left(\left(\kappa_{j}\right) H^{j}\right)$ where $p_{0}, p_{1}, p_{2} \in \mathbb{Z}$, we have that the associated 1-periodic orbits of at least two of them have distinct images in $M$. Define $Q_{i, j}:=H F_{\breve{C}, a_{-}^{j}, a_{+}^{j}}^{*}\left(\left(\kappa_{j}\right) \check{H}^{i, j}\right)$ for all $i \in \mathbb{N}$ and $j=0,1,2$. Then the pair of pants product is the natural composition

$$
\begin{aligned}
& \Phi_{H^{0}, H^{1}, H^{2}}: H F_{\check{C}, a_{-}^{0}, a_{+}^{0}}^{*}\left(H^{0}\right) \otimes_{\mathbb{K}} H F_{\breve{C}, a_{-}^{1}, a_{+}^{1}}^{*}\left(H^{1}\right)= \\
& \left(\underset{i \in \mathbb{N}}{\lim _{i}} Q_{i, 0}\right) \otimes_{\mathbb{K}}\left(\underset{i \in \mathbb{N}}{\lim _{i \in}} Q_{i, 1}\right) \longrightarrow \underset{i \in \mathbb{N}}{\lim _{i}}\left(Q_{i, 0} \otimes_{\mathbb{K}} Q_{i, 1}\right) \stackrel{\alpha}{\longrightarrow} \underset{i \in \mathbb{N}}{\lim _{\vec{N}}} Q_{i, 2}=H F_{\breve{C}, a_{-}^{2}, a_{+}^{2}}^{*}\left(2 H^{2}\right)
\end{aligned}
$$

where the morphism $\alpha$ is the direct limit of the natural pair of pants product map $Q_{i, 0} \otimes_{\mathbb{K}}$ $Q_{i, 1} \longrightarrow Q_{i, 2}$ as in Definition 2.85.

Remark 3.13. If $\left(\kappa_{j}\right) H^{j} \in \mathcal{H}^{\mathrm{reg}}\left(\check{C}, a_{-}^{j}, a_{+}^{j}\right)$ for $j=0,1,2$ then the pair of pants product map above is identical to the one defined in Definition 2.85 by Lemma 3.4. Also action maps and continuation maps commute with pair of pants product maps. Such a product is associative and graded commutative by Remark 2.86. If the domains of $a_{+}^{0}$ and $a_{+}^{1}$ agree then this product is $\Lambda^{Q_{+},{ }^{+}}$-bilinear where $Q_{+}$is the domain of $a_{+}^{0}$. If the constant Hamiltonian $\min \left(H^{0}\right)$ satisfies $\min \left(H^{0}\right) \leq_{\tilde{C}}^{\text {l.s. }} H^{0}$, if $a_{-}^{0}\left([\widetilde{\omega}], \lambda^{-}, \lambda^{+}\right)<\lambda^{-} \min \left(H^{0}\right)$ for all $\left([\widetilde{\omega}], \lambda^{-}, \lambda^{+}\right) \in Q_{0}-0$ and if $p_{0}=0$ then it has a left unit $\mathbf{1} \in H F_{\widetilde{C}, a_{-}^{0}, a_{+}^{0}}^{p_{0}}\left(H^{0}\right)$ by Remark 2.86 combined with the fact that continuation maps send left units to left units and the fact that $H F_{\check{C}, a_{-}^{0}, a_{+}^{0}}^{*}(K)$ has a left unit for any sufficiently $C^{2}$ small perturbation of $\min \left(H^{0}\right)$. By left unit we mean that for each $H^{3} \in \mathcal{H}^{\mathbb{T}, 1 . s .}\left(\check{C}, a_{-}^{2}, a_{+}^{2}\right)$ satisfying $2 H^{2}, H^{2} \leq \leq_{\check{C}}^{\text {l.s. }} H^{3}, \Phi_{2 H^{2}, H^{3}}^{p_{1}}\left(\Phi_{H^{0}, H^{1}, H^{2}}(\mathbf{1} \otimes x)\right)$ is equal to the image of $x$ under the composition

$$
H F_{\check{C}, a_{-}^{1}, a_{+}^{1}}^{p_{1}}\left(H^{1}\right) \longrightarrow H F_{\check{C}, a_{-}^{1}, a_{+}^{1}}^{p_{1}}\left(H^{3}\right) \longrightarrow H F_{\check{C}, a_{-}^{2}, a_{+}^{2}}^{p_{0}+p_{1}}\left(H^{3}\right)
$$

of action maps and continuation maps for each $x \in H F_{\check{C}, a_{-}^{1}, a_{+}^{1}}^{p_{1}}\left(H^{1}\right)$. This follows from Remark 2.86. We have a right unit under similar conditions. The only difference is that the indexes ' 0 ' and ' 1 ' are swapped.

\section{Definition of Symplectic Cohomology}

Throughout this section we will fix a contact cylinder $\check{C}=[1-\epsilon, 1+\epsilon] \times C \subset M$ with associated Liouville domain $D$.

Definition 4.1. Let $K \subset \mathbb{T} \times M$ be a closed set with the property that $K \subset \mathbb{T} \times D$ if $D$ is non-empty. We define $\mathcal{H}^{\mathbb{T}, 1 . s .}(\breve{C}, K, \leq 0)$ to be the subset of $\mathcal{H}^{\mathbb{T}, 1 . s .}(\check{C})$ (Definition 3.1) consisting of lower semi-continuous Hamiltonians $H=\left(H_{t}\right)_{t \in \mathbb{T}}$ with the property that $H_{t}(x) \leq 0$ if $(t, x) \in K$ and $H_{t}(x)=\infty$ otherwise. We define $\mathcal{H}^{\mathbb{T}, \text { l.s. }}(\check{C}, \leq 0)$ to be the union of 
all such subsets $\mathcal{H}^{\mathbb{T}, 1 . s .}(\check{C}, K, \leq 0)$. For each $H \in \mathcal{H}^{\mathbb{T}, \text { l.s. }}(\check{C}, \leq 0)$ and each $\check{C}$-interval domain $\left(Q_{-}, Q_{+}\right)$as in Definition 2.58, define $\mathbb{S} H_{\check{C}, Q_{-}, Q_{+}}^{*}(H)$ to be the double system of $\mathbb{Z}$-graded $\Lambda_{\mathbb{K}}^{Q_{+},{ }^{+}}$-modules (as in Definition 2.28):

$$
\mathbb{S} H_{\breve{C}, Q_{-}, Q_{+}}^{*}(H)=\left(H F_{\breve{C}, a_{-}, a_{+}}^{*}(H)\right)_{\left(a_{-}, a_{+}\right) \in \operatorname{Sc}\left(Q_{-}\right) \times \operatorname{Sc}\left(Q_{+}\right)}
$$

where

- the ordering on $\operatorname{Sc}\left(Q_{ \pm}\right)$is given by $\geq$,

- the double system morphisms are action maps as in Definition 3.7.

We define symplectic cohomology of $H$ to be $S H_{\check{C}, Q_{-}, Q_{+}}^{*}(H):=\underline{\lim } \underset{\longleftrightarrow}{\longleftrightarrow} \mathbb{S} H_{\check{C}, Q_{-}, Q_{+}}^{*}(H)$ where $\lim \lim$ is given in Definition 2.41. For any closed set $K \subset \mathbb{T} \times M$ which is contained in $\mathbb{T} \times D$

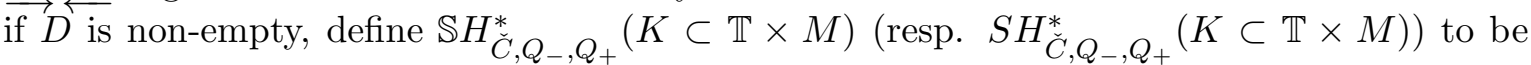
$\mathbb{S} H_{\breve{C}, Q_{-}, Q_{+}}^{*}\left(H_{K}\right)\left(\right.$ resp. $\left.S H_{\check{C}, Q_{-}, Q_{+}}^{*}\left(H_{K}\right)\right)$ where

$$
H_{K}=\left(H_{K, t}\right)_{t \in \mathbb{T}}, \quad H_{K, t}: M \longrightarrow \mathbb{R} \cup\{\infty\} \quad H_{K, t}(x):= \begin{cases}0 & \text { if }(t, x) \in K \\ \infty & \text { otherwise. }\end{cases}
$$

The algebra $S H_{C, Q_{-}, Q_{+}}^{*}(K \subset \mathbb{T} \times M)$ is called symplectic cohomology of $K$ in $M$.

If $K \subset M$ is a closed subset, which is contained in $D$ if $D$ is non-empty, then we define $\mathbb{S} H_{\check{C}, Q_{-}, Q_{+}}^{*}(K \subset M):=\mathbb{S} H_{\check{C}, Q_{-}, Q_{+}}^{*}(\mathbb{T} \times K \subset \mathbb{T} \times M)$ and $S H_{\breve{C}, Q_{-}, Q_{+}}^{*}(K \subset M):=$ $S H_{\overparen{C}, Q_{-}, Q_{+}}^{*}(\mathbb{T} \times K \subset \mathbb{T} \times M)$.

Remark 4.2. The definition of symplectic cohomology makes sense because $\mathcal{H}^{\mathbb{T}, \text { l.s. }}(\check{C}, \leq 0) \subset$ $\mathcal{H}^{\mathbb{T}, l . s .}\left(\check{C}, a_{-}, a_{+}\right)$for all $\check{C}$ action intervals $\left(a_{-}, a_{+}\right) \in \operatorname{Sc}\left(Q_{-}\right) \times \operatorname{Sc}\left(Q_{+}\right)$.

If the Hamiltonian $H$ is autonomous then $\mathbb{S} H_{\breve{C}, Q_{-}, Q_{+}}^{*}(H)$ is a double system of $\mathbb{Z}$-graded $\Lambda_{\mathbb{K}}^{Q_{+},{ }^{+}}$-modules with product induced by the pair of pants product maps

$$
H F_{\check{C}, a_{-}^{0}, a_{+}^{0}}^{*}(H) \otimes_{\Lambda_{\mathbb{K}}^{Q_{+},+}} H F_{\check{C}, a_{-}^{1}, a_{+}^{1}}^{*}(H) \longrightarrow H F_{\check{C}, a_{-}^{2}, a_{+}^{2}}^{*}(H)
$$

where $a_{-}^{2}=a_{-}^{0}+a_{-}^{1}, a_{+}^{2}=\min \left(a_{-}^{0}+a_{+}^{1}, a_{-}^{1}+a_{+}^{0}\right)$. This product is well defined since $H$ is autonomous and $H \leq \leq_{\check{C}}^{\text {l.s. }} \frac{1}{2} H$ because the slope of $H$ along $\check{C}$ is 0 and because $H$ is either non-positive or $\infty$. Also $S H_{\check{C}, Q_{-}, Q_{+}}^{*}(H)$ is a graded $\Lambda_{\mathbb{K}}^{Q_{+},+}$-algebra by Remark 2.45. Such an algebra is a unital graded commutative algebra by Remark 3.13.

Definition 4.3. Let $\left(Q_{-}, Q_{+}\right)$be a $\check{C}$-interval domain pair. Let $H^{ \pm} \in \mathcal{H}^{\mathbb{T}, 1 . s .}(\check{C}, \leq 0)$ satisfy $H^{-} \leq H^{+}$. Then $H_{-} \leq_{\check{C}} H_{+}$and hence the natural continuation maps

$$
H F_{\breve{C}, a_{-}, a_{+}}^{*}\left(H_{-}\right) \longrightarrow H F_{\breve{C}, a_{-}, a_{+}}^{*}\left(H_{+}\right)
$$

for all $\check{C}$-action intervals $\left(a_{-}, a_{+}\right)$give us a morphism of double systems

$$
\mathbb{S} H_{C, Q_{-}, Q_{+}}^{*}\left(H_{-}\right) \longrightarrow \mathbb{S} H_{\breve{C}, Q_{-}, Q_{+}}^{*}\left(H_{+}\right)
$$

called a transfer morphism. This also induces a morphism of algebras $S H_{\overparen{C}, Q_{-}, Q_{+}}^{*}\left(H_{-}\right) \longrightarrow$ $S H_{\check{C}, Q_{-}, Q_{+}}^{*}\left(H_{+}\right)$called a transfer map. In particular, if $K_{+} \subset K_{-} \subset D$ are closed subsets, we have a transfer map $S H_{\check{C}, Q_{-}, Q_{+}}^{*}\left(K_{-} \subset M\right) \longrightarrow S H_{\check{C}, Q_{-}, Q_{+}}^{*}\left(K_{+} \subset M\right)$.

If $H_{ \pm}$is autonomous then this transfer morphism or map respects the natural product structures on the corresponding double systems or modules and they also send units to units. 
Definition 4.4. Let $\left(Q_{-}^{j}, Q_{+}^{j}\right)$ be a $\check{C}$-interval domain pair for $j=0,1$ so that $Q_{ \pm}^{1} \subset Q_{ \pm}^{0}$. Suppose $H \in \mathcal{H}^{\mathbb{T}}$,l.s. $(\check{C}, \leq 0)$. Then the action maps

$$
H F_{\breve{C}, a_{-}, a_{+}}^{*}(H) \longrightarrow H F_{\breve{C},\left.a_{-}\right|_{Q_{-}^{1}},\left.a_{+}\right|_{Q_{+}^{1}}}^{*}(H), \quad\left(a_{-}, a_{+}\right) \in \operatorname{Sc}\left(Q_{-}^{0}\right) \times \operatorname{Sc}\left(Q_{+}^{0}\right)
$$

give us a morphism of double systems $\mathbb{S} H_{\breve{C}, Q_{-}^{0}, Q_{+}^{0}}^{*}(H) \longrightarrow \mathbb{S} H_{\check{C}, Q_{-}^{1} \cdot Q_{+}^{1}}^{*}(H)$ called an action morphism.

Again this morphism respects the product structure if $H$ is autonomous.

Definition 4.5. Let $\left(Q_{-}, Q_{+}\right)$be a $\check{C}$-interval domain pair and let $H \in \mathcal{H}^{\mathbb{T}, \text { l.s. }}(\check{C}, \leq 0)$. Let $F: \mathbb{T} \longrightarrow \mathbb{T}$ be a smooth non-decreasing map. Then the reparameterization isomorphisms

$$
H F_{\check{C}, a_{-}, a_{+}}^{*}(H) \cong H F_{\check{C}, a_{-}, a_{+}}^{*}\left(H^{F}\right)
$$

from Definition 3.11 for each $\check{C}$-action interval $\left(a_{-}, a_{+}\right) \in \operatorname{Sc}\left(Q_{-}\right) \times \operatorname{Sc}\left(Q_{+}\right)$gives us an isomorphism of double systems

$$
\mathbb{S} H_{\breve{C}, Q_{-}, Q_{+}}^{*}(H) \cong \mathbb{S} H_{\breve{C}, Q_{-}, Q_{+}}^{*}\left(H^{F}\right)
$$

called a reparameterization isomorphism.

Lemma 4.6. Let $\left(Q_{-}, Q_{+}\right)$be a $\check{C}$-interval domain pair and let $H \in \mathcal{H}^{\mathbb{T}, \text { l.s. }}(\check{C}, \leq 0)$. Then

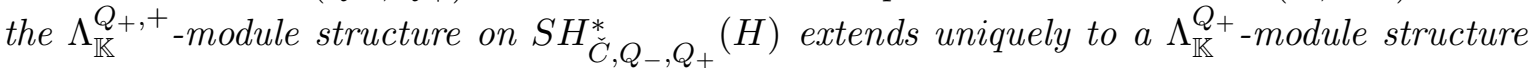
on $S H_{\overparen{C}, Q_{-}, Q_{+}}^{*}(H)$ making it into a $\Lambda_{\mathbb{K}}^{Q_{+}}$-algebra.

Proof. Let $\pi_{D}: H^{2}(M, D ; \mathbb{R}) \times \mathbb{R} \times \mathbb{R} \longrightarrow H^{2}(M, D ; \mathbb{R})$ be the natural projection map and let $\iota_{D}: H^{2}(M, D ; \mathbb{R}) \longrightarrow H^{2}(M ; \mathbb{R})$ be the natural restriction map. Let

$$
Q:=\iota_{D}\left(\pi_{D}\left(Q_{+}\right)\right) \subset H^{2}(M ; \mathbb{R})=\left(H_{2}(M ; \mathbb{Z}) \otimes_{\mathbb{Z}} \mathbb{R}\right)^{*}
$$

and let $\preceq_{Q}$ be the induced ordering on $H_{2}(M ; \mathbb{Z})$ as in Definition 2.52. Define $Q^{\vee}:=\{x \in$ $\left.H_{2}(M ; \mathbb{Z}): 0 \preceq_{Q} x\right\} \subset H_{2}(M ; \mathbb{Z})$. By Definition 2.56 , we have that $Q^{\vee}$ is naturally a submonoid of the multiplicative group $\left(\Lambda_{\mathbb{K}}^{Q_{+},+}\right)^{\times}$. All such elements are invertible in $\Lambda_{\mathbb{K}}^{Q_{+}}$. Also each element $w \in \Lambda_{\mathbb{K}}^{Q_{+}}$has the property that there exists some element $s_{w} \in Q^{\vee}$ satisfying $s_{w} w \in \Lambda_{\mathbb{K}}^{Q_{+},+}$. Therefore by [Sta18, Tag 07JY] it is sufficient for us to show that each $s \in Q^{\vee}$ acts as an automorphism on $S H_{\breve{C}, Q_{-}, Q_{+}}^{*}(H)$.

We now fix such an element $s$. Since $s \in H_{2}(M ; \mathbb{Z})$, it defines a linear function on $H^{2}(M ; \mathbb{R})$ and hence by pulling back via $\iota_{D}$ and $\pi_{D}$ and restricting to $Q_{+}$, a function $L_{s} \in \operatorname{Sc}\left(Q_{+}\right)$. The map sending a capped loop $\gamma$ to $\gamma \#(-s)$ induces an isomorphism $H F_{\breve{C}, a_{-}-L_{s}, a_{+}-L_{s}}^{*}(H) \cong$ $H F_{\breve{C}, a_{-}, a_{+}}^{*}(H)$. Since $S H_{\breve{C}, Q_{-}, Q_{+}}^{*}(H)=\lim _{a_{-}} \lim _{a_{+}} H F_{\check{C}, a_{-}-L_{s}, a_{+}-L_{s}}^{*}(H)$, we get that such a map induces an automorphism of $S H_{\breve{C}, Q_{-}, Q_{+}}^{*}(H)$. This automorphism coincides with the natural module action of $s \in \Lambda_{\mathbb{K}}^{Q_{+},+}$. Hence the $\Lambda_{\mathbb{K}}^{Q_{+},+}$-action extends to a $\Lambda_{\mathbb{K}}^{Q_{+}}$-action.

Remark 4.7. Since continuation maps and action maps between symplectic cohomology algebras of autonomous Hamiltonians are induced by inclusions of double systems, they are

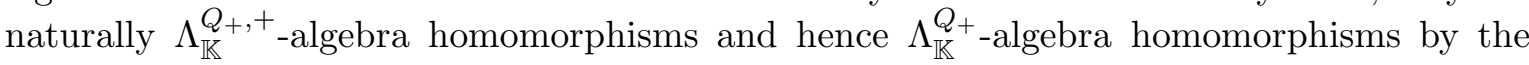
lemma above. 


\section{Properties of Symplectic Cohomology}

\subsection{Changing Contact Cylinders.}

Proposition 5.1. Let $\check{C}_{j}$ be a contact cylinder with associated Liouville domain $D_{j}$ for $j=$ 0,1 so that $D_{1} \subset D_{0}$. Suppose also that the natural restriction map $\iota:=H^{2}\left(M, D_{0} ; \mathbb{R}\right) \longrightarrow$ $H^{2}\left(M, D_{1} ; \mathbb{R}\right)$ is an isomorphism and define

$$
\widetilde{\iota}: H^{2}\left(M, D_{0} ; \mathbb{R}\right) \times \mathbb{R}^{2} \longrightarrow H^{2}\left(M, D_{1} ; \mathbb{R}\right) \times \mathbb{R}^{2}, \quad \widetilde{\iota}:=\iota \times \operatorname{id}_{\mathbb{R}^{2}} .
$$

Let $\left(Q_{-}, Q_{+}\right)$be a wide $\check{C}_{0}$-interval domain and let $H \in \mathcal{H}^{\mathbb{T}, \text { l.s. }}\left(\check{C}_{1}, \leq 0\right)$ be autonomous. Then there is an isomorphism of double systems with product

$$
\mathbb{S} H_{\check{C}_{0}, Q_{-}, Q_{+}}^{*}(H) \stackrel{\cong}{\longrightarrow} \mathbb{S} H_{\check{C}_{1}, \widetilde{\iota}\left(Q_{-}\right), \widetilde{\iota}\left(Q_{+}\right)}^{*}(H) .
$$

Before we prove this proposition, we need a preliminary lemma.

Lemma 5.2. Proposition 5.1 is true when $\check{C}_{0}$ and $\check{C}_{1}$ are disjoint

Proof of lemma 5.2. Let $\left(a_{-}^{j}\right)_{j \in \mathbb{N}},\left(a_{+}^{j}\right)_{j \in \mathbb{N}}$ be a cofinal family of $\left(\operatorname{Sc}\left(Q_{-}^{0}\right), \geq\right)$ and $\left(\operatorname{Sc}\left(Q_{+}^{0}\right), \leq\right.$ ) respectively. After passing to a subsequence, we can assume that the function $i \rightarrow$ height $\left(a_{-}^{i}, a_{+}^{i}\right)$ is increasing.

Since $H$ has slope 0 along both contact cylinders and $\check{C}_{0} \cap \check{C}_{1}=\emptyset$ we can find a cofinal family of Hamiltonians $H_{i} \in \cap_{k=0,1} \cap_{j \in \mathbb{N}} \mathcal{H}^{\mathbb{T}, \text { reg }}\left(<_{\check{C}_{k}} H, a_{-}^{j}, a_{+}^{j}\right), i \in \mathbb{N}$ so that $\left.H_{i}\right|_{M-D_{1}}$ is constant and $\left.H_{i}\right|_{M-D_{1}}>\operatorname{height}\left(a_{-}^{i}, a_{+}^{i}\right)$ for all $i \in \mathbb{N}$. Then the Floer chain complexes computing $H F_{\breve{C}_{1}, a_{-}^{j}, a_{+}^{j}}^{*}\left(H_{i}\right)$ and $H F_{\breve{C}_{0}, a_{-}^{j}, a_{+}^{j}}^{*}\left(H_{i}\right)$ are identical for each $i, j \in \mathbb{N}$ satisfying $i \geq j$ by Corollary 2.8. The continuation maps, action maps and pair of pants product maps coincide under such an isomorphism. This gives us our isomorphism (5.2) by Lemma 2.40 .

Proof of Proposition 5.1. Let

$$
\check{C}_{j}=\left[1-\epsilon_{j}, 1+\epsilon_{j}\right] \times C_{j}, \quad j=0,1
$$

be our contact cylinders. By a Moser argument ([MS98, Exercise 3.36]), we can extend them to contact cylinders

$$
\check{C}_{j}^{\prime}=\left[1-\epsilon_{j}-\delta, 1+\epsilon_{j}+\delta\right] \times C_{j}, \quad j=0,1
$$

for some $0<\delta<\min _{j=0,1}\left(\epsilon_{j} / 2\right)$. We can also assume that $\delta$ is small enough so that $[1-\delta, 1+\delta] \times C_{1}$ is disjoint from $\left[1+\epsilon_{0}+\delta / 2,1+\epsilon_{0}+\delta\right] \times C_{0}$. We now apply Lemma 5.2 four times, with four different pairs of contact cylinders in the following order:

(1) $\check{C}_{0},\left[1+\epsilon_{0}+\delta / 2,1+\epsilon_{0}+\delta\right] \times C_{0}$,

(2) $\left[1+\epsilon_{0}+\delta / 2,1+\epsilon_{0}+\delta\right] \times C_{0},[1-\delta, 1+\delta] \times C_{1}$,

(3) $[1-\delta, 1+\delta] \times C_{1},\left[1+\epsilon_{1}+\delta / 2,1+\epsilon_{1}+\delta\right] \times C_{1}$ and

(4) $\left[1+\epsilon_{1}+\delta / 2,1+\epsilon_{1}+\delta\right] \times C_{1}, \check{C}_{1}$.

This completes the proposition.

We also need a proposition telling us what to do when we forget the contact cylinder.

Definition 5.3. Let $\check{C}=\emptyset$ be the empty contact cylinder. The standard $\omega$-cone is the cone $Q_{\omega} \subset H^{2}(M, \emptyset ; \mathbb{R}) \times \mathbb{R} \times \mathbb{R}$ defined to be the 1 -dimensional cone spanned by $([\omega], 1,1)$. The standard Novikov ring $\Lambda_{\mathbb{K}}^{\omega}$ is defined to be $\Lambda_{\mathbb{K}}^{Q_{\omega}}$. The standard positive Novikov ring $\Lambda_{\mathbb{K}}^{\omega,+}$ is defined to be $\Lambda_{\mathbb{K}}^{Q_{\omega},+}$. 
Note that the standard Novikov ring is equal to the Novikov ring (1.2) in the introduction with $\omega_{X}$ replaced by $\omega$.

Proposition 5.4. Let $\check{C}=[1-\epsilon, 1+\epsilon] \times C$ be a contact cylinder with associated Liouville domain $D$. Let $Q_{\omega_{\check{C}}} \subset H^{2}(M, D ; \mathbb{R}) \times \mathbb{R}^{2}$ be the cone spanned by $\left(\left[\omega_{\check{C}}\right], 1,1\right)$ where $\omega_{\check{C}}$ is a $\check{C}$-compatible 2 -form with scaling constants 0 and 1 and which is equal to $\omega$ outside $D \cup([1,1+\epsilon / 2] \times C)$. Then for any autonomous $H \in \mathcal{H}^{\mathbb{T}, \text { l.s. }}(\check{C}, \leq 0)$ (Definition 4.1) there is an isomorphism of double systems with product

$$
\mathbb{S} H_{\check{C}, Q_{\omega_{\check{C}}}, Q_{\omega_{\check{C}}}}^{*}(H) \stackrel{\cong}{\longrightarrow} \mathbb{S} H_{\emptyset, Q_{\omega}, Q_{\omega}}^{*}(H) .
$$

Proof. Let $\Pi: Q_{\omega_{\check{C}}} \longrightarrow Q_{\omega}$ be the restriction of the natural map $H^{2}(M, D ; \mathbb{R}) \times \mathbb{R}^{2} \longrightarrow$ $H^{2}(M ; \mathbb{R}) \times \mathbb{R}^{2}$ induced by cohomological restriction. Choose any cofinal family of Hamiltonians $H_{i} \in \mathcal{H}^{\mathbb{T}, \text { reg }}\left(<_{\check{C}} H, a_{-}, a_{+}\right)$. Then the isomorphism (5.3) follows from the natural isomorphisms $H F_{\breve{C}, a_{-} \circ \Pi, a_{+} \circ \Pi}^{*}(H) \cong H F_{\emptyset, a_{-}, a_{+}}^{*}(H)$ coming from the fact that the corresponding Floer chain complexes are identical for each $\left(a_{-}, a_{+}\right) \in \operatorname{Sc}\left(Q_{\omega}\right) \times \operatorname{Sc}\left(Q_{\omega}\right)$.

5.2. Partial Independence of the Hamiltonian. Throughout this subsection, $\check{C}$ is a contact cylinder whose associated Liouville domain is $D$. We will also fix a $\check{C}$-interval domain $\left(Q_{-}, Q_{+}\right)$.

Proposition 5.5. Let $K \subset \mathbb{T} \times M$ be a closed subset which is contained in $D$ if $D$ is nonempty. Let $H^{ \pm} \in \mathcal{H}^{\mathbb{T}, \text { l.s. }}(\check{C}, K, \leq 0)$ satisfy $H_{t}^{-} \leq H_{t}^{+}$. Then the transfer morphism

$$
\mathbb{S} H_{\check{C}, Q_{-}, Q_{+}}^{*}\left(H^{-}\right) \longrightarrow \mathbb{S} H_{\check{C}, Q_{-}, Q_{+}}^{*}\left(H^{+}\right)
$$

is an isomorphism in the category 2 -sys- $\Lambda_{\mathbb{K}}^{Q_{+},+}$.

Since $\lim _{\longrightarrow} \lim$ is a functor by Definition 2.41, Proposition 5.5 implies that the transfer map $S H_{\breve{C}, Q_{-}, Q_{+}}^{*}(\check{H}) \longrightarrow S H_{\breve{C}, Q_{-}, Q_{+}}^{*}(H)$ is an isomorphism. Before we prove Proposition 5.5, we need some preliminary lemmas and the following definition.

Definition 5.6. Let $\nu \in \mathbb{R}$ be a constant. Define

$$
L_{\nu}: H^{2}(M, D ; \mathbb{R}) \times \mathbb{R} \times \mathbb{R} \longrightarrow \mathbb{R}, \quad L_{\nu}\left(q, \lambda^{-}, \lambda^{+}\right):=\lambda^{-} \nu .
$$

Lemma 5.7. Let $\left(a_{-}, a_{+}\right) \in \operatorname{Sc}\left(Q_{-}\right) \times \operatorname{Sc}\left(Q_{+}\right)$be a $\check{C}$-action interval, $\nu \in(0, \infty)$ and let $H \in \mathcal{H}^{\mathbb{T}, \text { l.s. }}\left(\check{C}, a_{-}+L_{\nu}, a_{+}+L_{\nu}\right)$. Then we have a commutative diagram:

$$
\begin{array}{r}
H F_{\breve{C}, a_{-}, a_{+}}^{*}(H-\nu) \stackrel{\alpha}{\longrightarrow} H F_{\left(\check{C}_{\left., a_{-}, a_{+}\right)}^{*}\right.}^{*}(H) \\
H F_{\check{C}, a_{-}+L_{\nu}, a_{+}+L_{\nu}}^{*}(H) \overbrace{\beta}^{\gamma}(H \cong
\end{array}
$$

where $\alpha$ is a continuation map and $\beta$ is an action map. The map $\gamma$ commutes with continuation maps and action maps.

Definition 5.8. We will call the map $\gamma$ a translation isomorphism.

Proof of Lemma 5.7. We will prove this lemma in two steps. In the first step, we will prove it in the case when $H \in \mathcal{H}^{\mathrm{reg}}\left(\check{C}, a_{-}+L_{\nu}, a_{+}+L_{\nu}\right)$ (Definition 2.58) and the second step will deal with the general case. 
Step 1. Suppose $H \in \mathcal{H}^{\mathrm{reg}}\left(\check{C}, a_{-}+L_{\nu}, a_{+}+L_{\nu}\right)$. For $N \in \mathbb{N}$ large enough, we have that $H-c \in \mathcal{H}^{\mathrm{reg}}\left(\check{C}, a_{-}+L_{\nu}, a_{+}+L_{\nu}\right)$ for all $c \in\left[0, \frac{2 \nu}{N}\right]$. Therefore $H-(N-k+s) \nu / N \in$ $\mathcal{H}^{\mathrm{reg}}\left(\check{C}, a_{-}+k L_{\nu} / N, a_{+}+k L_{\nu} / N\right)$ for all $k \in \mathbb{Z}$ and all $s \in[0,1]$. Define

$$
B_{k, l}:=H F_{\breve{C}, a_{-}+k L_{\nu} / N, a_{+}+k L_{\nu} / N}^{*}(H-(N-l) \nu / N)
$$

for all $l, k \in\{0, \cdots, N\}$. Then by Lemmas 2.74 and 2.79 we have a commutative diagram consisting of continuation maps and action maps (with the exception of $\gamma$ which is given as a definition):

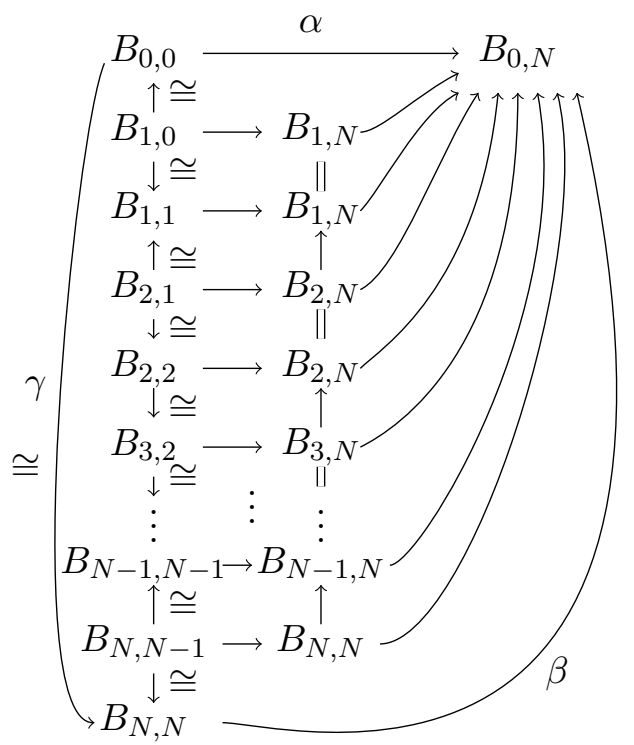

The map $\gamma$ commutes with continuation maps and action maps since the diagram above is functorial with respect to continuation and action maps.

Step 2: Now suppose $H \in \mathcal{H}^{\mathbb{T}}$,l.s. $\left(\check{C}, a_{-}+L_{\nu}, a_{+}+L_{\nu}\right)$. Then since

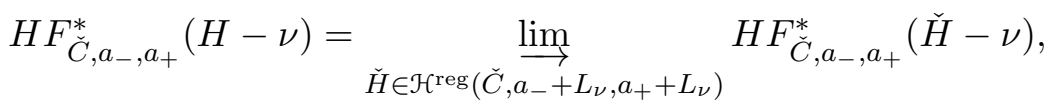

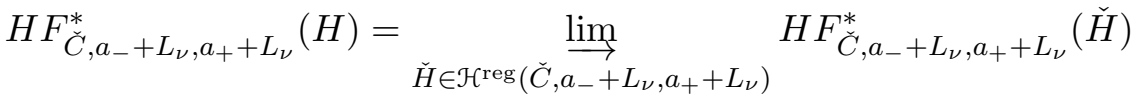

and continuation maps commute with the translation isomorphisms constructed in Step 1, we get our result.

Lemma 5.9. Let $H \in \mathcal{H}^{\mathbb{T}, 1 . s .}(\check{C}, \leq 0)$ and let $\nu>0$ be a constant. Then the transfer morphism $\mathbb{S} H_{\breve{C}, Q_{-}, Q_{+}}^{*}(H-\nu) \longrightarrow \mathbb{S} H_{\check{C}, Q_{-}, Q_{+}}^{*}(H)$ is an isomorphism in 2 -sys- $\Lambda_{\mathbb{K}}^{Q_{+},+}$.

Proof. For each action interval $\left(a_{-}, a_{+}\right) \in \operatorname{Sc}\left(Q_{-}\right) \times \operatorname{Sc}\left(Q_{+}\right)$, we have a translation isomorphism

$$
\gamma_{a_{-}, a_{+}}: H F_{\breve{C}, a_{-}, a_{+}}^{*}(H-\nu) \longrightarrow H F_{\breve{C}, a_{-}+L_{\nu}, a_{+}+L_{\nu}}^{*}(H)
$$


Since such isomorphisms commute with continuation maps, we get an isomorphism

$$
\gamma: \mathbb{S} H_{\breve{C}, Q_{-}, Q_{+}}^{*}(H-\nu) \longrightarrow \mathbb{S} H_{\breve{C}, Q_{-}, Q_{+}}^{*}(H) .
$$

Also for each action interval $\left(a_{-}, a_{+}\right) \in \operatorname{Sc}\left(Q_{-}\right) \times \operatorname{Sc}\left(Q_{+}\right)$, we have an action morphism

$$
\beta_{a_{-}, a_{+}}: H F_{\breve{C}, a_{-}+L_{\nu}, a_{+}+L_{\nu}}^{*}(H) \longrightarrow H F_{\breve{C}, a_{-}, a_{+}}^{*}(H)
$$

and this induces an isomorphism

$$
\beta: \mathbb{S} H_{\check{C}, Q_{-}, Q_{+}}^{*}(H) \longrightarrow \mathbb{S} H_{\check{C}, Q_{-}, Q_{+}}^{*}(H)
$$

(such an isomorphism is induced by a standard endomorphism as in Example 2.37). Also by Lemma 5.7,

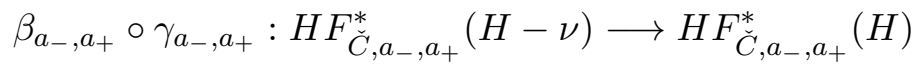

is a continuation map for all $a_{-}, a_{+} \in \operatorname{Sc}(Q)$ giving us a transfer morphism

$$
\mathbb{S} H_{\breve{C}, Q_{-}, Q_{+}}^{*}(H-\nu) \longrightarrow \mathbb{S} H_{\check{C}, Q_{-}, Q_{+}}^{*}(H) \text {. }
$$

Our lemma now follows from the fact that $\beta \circ \gamma$ is a composition of isomorphisms in 2 -sys- $\Lambda_{\mathbb{K}}^{Q,+}$.

Proof of Proposition 5.5. Choose a constant $\nu>0$ so that $H^{+}-\nu \leq H^{-}$. Then since $H^{ \pm} \in \mathcal{H}^{\mathbb{T}, \text { l.s. }}(\check{C}, \leq 0)$ we get $H^{+}-\nu \leq_{\check{C}} H^{-}$. We then have transfer morphisms

$\mathbb{S} H_{\breve{C}, Q_{-}, Q_{+}}^{*}\left(H^{+}-2 \nu\right) \longrightarrow \mathbb{S} H_{\check{C}, Q_{-}, Q_{+}}^{*}\left(H^{-}-\nu\right) \stackrel{\alpha}{\longrightarrow} \mathbb{S} H_{\check{C}, Q_{-}, Q_{+}}^{*}\left(H^{+}-\nu\right) \longrightarrow \mathbb{S} H_{\check{C}, Q_{-}, Q_{+}}^{*}\left(H^{-}\right)$.

By Lemma 5.9, the composition of any two such morphisms is an isomorphism and hence $\alpha$ is an isomorphism. Also by Lemma 5.9, the natural continuation morphisms

$$
\beta_{ \pm}: \mathbb{S} H_{\overparen{C}, Q_{-}, Q_{+}}^{*}\left(H^{ \pm}-\nu\right) \longrightarrow \mathbb{S} H_{\overparen{C}, Q_{-}, Q_{+}}^{*}\left(H^{ \pm}\right)
$$

are isomorphisms. Our Proposition now follows from the fact that the continuation map (5.4) is equal to the following composition of isomorphisms: $\beta_{+} \circ \alpha \circ\left(\beta_{-}\right)^{-1}$.

\subsection{Relation with Quantum Cup Product.}

Theorem 5.10. Let $Q_{\omega}, \Lambda_{\mathbb{K}}^{\omega}$ be the standard $\omega$-cone and standard Novikov ring respectively as in Definition 5.3. Suppose that $\mathbb{K}$ is a field. Then there is an isomorphism of $\Lambda_{\mathbb{K}}^{\omega}$-algebras

$$
S H_{\emptyset, Q_{\omega}, Q_{\omega}}^{*}(M \subset M) \cong Q H^{*}\left(M, \Lambda_{\mathbb{K}}^{\omega}\right)
$$

where $Q H^{*}$ is quantum cohomology. Also, $\underline{\lim }_{\longleftrightarrow} \underset{\lim ^{1}}{\leftarrow} \mathbb{S} H_{\emptyset, Q_{\omega}, Q_{\omega}}^{*}(M \subset M)=0$ where $\stackrel{\lim \lim ^{1}}{\longleftarrow}$ is given in Definition 2.50 .

Proof. We identify the cone $Q_{\omega}$ with $[0, \infty)$ via the identification $\lambda([\omega], 1,1) \longrightarrow \lambda$ for all $\lambda \geq 0$. Under this identification, $\operatorname{Sc}\left(Q_{\omega}\right)$ becomes the space of linear functions $a_{c}:[0, \infty) \longrightarrow$ $\mathbb{R}, \quad a_{c}(\lambda):=c \lambda, c \in \mathbb{R}$.

We will first show $\lim _{\longrightarrow} \lim ^{1} \mathbb{S} H_{\emptyset, Q_{\omega}, Q_{\omega}}^{*}(M \subset M)=0$ by using Lemma 2.51. Let $H: M \longrightarrow \mathbb{R}$ be a $C^{2}$ small Morse function which is negative. Since $[\omega]$ lifts to an integral cohomology class in $H^{2}(M ; \mathbb{Z})$, we have for all $c_{ \pm} \in \frac{1}{2}+\mathbb{Z}$ that the continuation maps

$$
H F_{\emptyset, a_{c_{-}}, a_{c_{+}}}^{*}\left(\lambda_{1} H\right) \longrightarrow H F_{\emptyset, a_{c_{-}}, a_{c_{+}}}^{*}\left(\lambda_{2} H\right)
$$

are isomorphisms for all $0<\lambda_{2} \leq \lambda_{1} \leq \frac{1}{4}$ by Lemma 2.74. Hence the double system $W:=\left(H F_{\emptyset, a_{c_{-}}, a_{c_{+}}}^{*}(\lambda H)\right)_{c_{ \pm} \in \frac{1}{2}+\mathbb{Z}}$ for some small $\lambda>0$ is isomorphic to the double system 


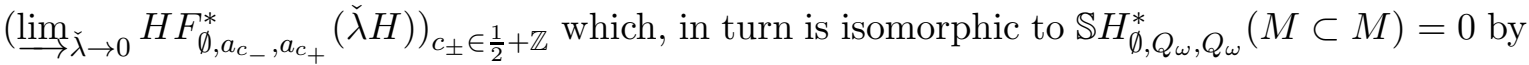
Lemma 2.40. Therefore by Lemma 2.51 it is sufficient for us to show that the action map

$$
H F_{\emptyset, a_{c_{-}}, a_{c_{+}}^{*}}^{*}(\lambda H) \longrightarrow H F_{\emptyset, a_{c_{-}}, a_{c_{+}-1}}^{*}(\lambda H)
$$

is surjective for each $c_{ \pm} \in \frac{1}{2}+\mathbb{Z}$. Since $\lambda H$ is $C^{2}$ small, we have by [AD14, Theorem 10.1.1] that the only Floer trajectories connecting 1-periodic orbits of $\lambda H$ are Morse flowlines (I.e. $t$ independent Floer trajectories). This implies that the natural map (5.6) is surjective for each $c_{ \pm} \in \frac{1}{2}+\mathbb{Z}$ which in turn implies that $\lim _{\longrightarrow} \lim ^{1} \mathbb{S} H_{\emptyset, Q_{\omega}, Q_{\omega}}^{*}(M \subset M)=0$ by Lemma 2.51.

We will now prove the first part of the lemma. Choose $J \in \cap_{c_{ \pm} \in \frac{1}{2}+\mathbb{Z}} \mathcal{J}^{\mathbb{T}, \text { reg }}\left(\lambda H, a_{c_{-}}, a_{c_{+}}\right)$. Define

$$
H F^{*}(\lambda H):=H_{*}\left(\underset{c_{-}}{\lim } \underset{c_{+}}{\lim } C F_{\emptyset, a_{c_{-}}, a_{c_{+}}}^{*}(\lambda H)\right) .
$$

Since $\lim \lim ^{1} W=0$, we have a natural isomorphism $H F^{*}(\lambda H)=\lim _{a_{-}} \lim _{a_{+}} H F_{\emptyset, a_{-}, a_{+}}^{*}(\lambda H)$ by [Wei94, Theorem 3.5.8] and Lemma 2.40. Since the morphism (5.5) is an isomorphism for all $c_{ \pm} \in \frac{1}{2} \mathbb{Z}$ and $0<\lambda_{2}<\lambda_{1} \leq \frac{1}{4}$, we get that the natural map

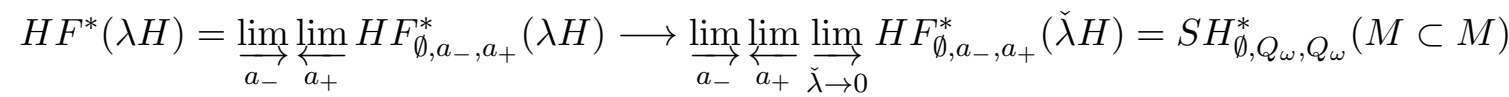

is an isomorphism for all sufficiently small $\lambda>0$. By [PSS96] and [OZ11] we have isomorphisms

$$
H F^{*}(\lambda H) \cong H^{*}\left(M ; \Lambda_{\mathbb{K}}^{\omega}\right)
$$

for all $\lambda>0$ small which commute with continuation maps $H F^{*}(\lambda H) \longrightarrow H F^{*}\left(\lambda^{\prime} H\right)$ for $0<\lambda<\lambda^{\prime}$ small. These isomorphisms also commute with the pair of pants product as follows. If $\lambda>0$ is sufficiently small, we have a commutative diagram of $\Lambda_{\mathbb{K}}^{\omega}$-modules

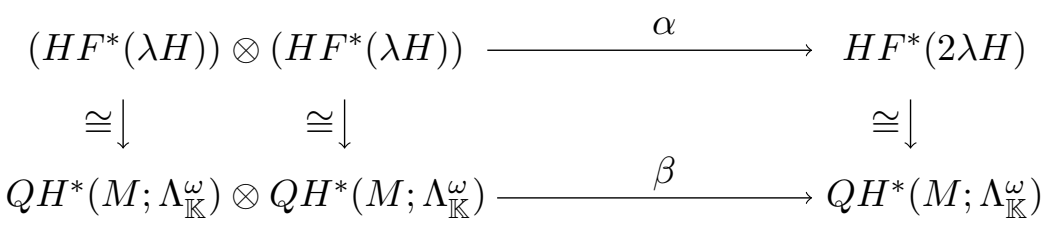

where $\alpha$ is the pair of pants product and $\beta$ is the quantum cup product and where the vertical isomorphisms are induced by (5.8) (the papers [PSS96] and [OZ11] only prove such isomorphisms over $\mathbb{Q}$, however these proofs are identical if one just formally replaces the coefficient field ' $\mathbb{Q}$ ' with ' $\mathbb{K}$ ' in their papers since $(M, \omega)$ is semi-positive). Therefore by Equation (5.7) we have an isomorphism of $\Lambda_{\mathbb{K}^{-}}^{\omega}$-algebras

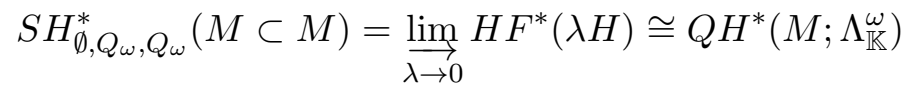

since the isomorphism (5.7) commutes with the pair of pants product $\alpha$.

\subsection{Stably Displaceable Complements.}

Definition 5.11. A subset $B$ of a symplectic manifold is Hamiltonian displaceable if there is a Hamiltonian symplectomorphism $\phi$ satisfying $\phi(B) \cap B=\emptyset$. A subset $A \subset M$ is stably displaceable if $A \times \mathbb{T} \subset M \times T^{*} \mathbb{T}$ is Hamiltonian displaceable inside the product symplectic 
manifold $M \times T^{*} \mathbb{T}$ where $T^{*} \mathbb{T}=\mathbb{R} \times \mathbb{T}$ has the standard symplectic form $d \sigma \wedge d \tau$ where $\sigma: \mathbb{R} \times \mathbb{T} \longrightarrow \mathbb{R}, \tau: \mathbb{R} \times \mathbb{T} \longrightarrow \mathbb{T}$ are the natural projection maps.

Throughout this subsection, we fix the coordinates $\sigma, \tau$ above. We also let $Q_{\omega}$ be the standard Novikov cone associated to the empty contact cylinder as in Definition 5.3. The aim of this subsection is to prove the following proposition.

Theorem 5.12. Let $K \subset M$ be a closed set so that $\overline{M-K}$ is stably displaceable. Then the transfer morphism

$$
\mathbb{S} H_{\emptyset, Q_{\omega}, Q_{\omega}}^{*}(M \subset M) \longrightarrow \mathbb{S} H_{\emptyset, Q_{\omega}, Q_{\omega}}^{*}(K \subset M)
$$

is an isomorphism in 2 -sys- $\Lambda_{\mathbb{K}}^{Q_{\omega},+}$.

The proof of this proposition relies heavily on an idea due to Ginzburg in [Gin10]. Before we prove this proposition, we need some preliminary definitions and lemmas.

Definition 5.13. A lower semi-continuous Hamiltonian $H=\left(H_{t}\right)_{t \in \mathbb{T}}$ on $M \times T^{*} \mathbb{T}$ is admissible if there is a compact subset $K_{H} \subset M \times T^{*} \mathbb{T}$ and a lower semi-continuous Hamiltonian $K=\left(K_{t}\right)_{t \in \mathbb{T}}$ on $M$ so that $H_{t}(x,(\sigma, \tau))=K_{t}(x)-\frac{1}{2}|\sigma|$ for all $(x,(\sigma, \tau)) \in M \times T^{*} \mathbb{T}-K_{H}$.

Let $J_{T^{*} \mathbb{T}}$ be the almost complex structure on $\mathbb{R} \times \mathbb{T}$ satisfying $J_{T^{*} \mathbb{T}}\left(\frac{\partial}{\partial \sigma}\right)=\frac{\partial}{\partial \tau}$. A smooth family of almost complex structures $J=\left(J_{t}\right)_{t \in \mathbb{T}}$ on $M \times T^{*} \mathbb{T}$ is admissible if they are $\omega+$ $d \sigma \wedge d \tau$-tame and if they are equal to $J_{M} \oplus J_{T^{*} \mathbb{T}}$ outside a compact subset of $M \times T^{*} \mathbb{T}$ where $J_{M}$ is an $\omega$-tame almost complex structure on $M$.

For any admissible lower semi-continuous Hamiltonian $H$ on $M \times T^{*} \mathbb{T}$ and any $a_{-}, a_{+} \in$ $\operatorname{Sc}\left(Q_{\omega}\right)$, we can define

$$
H F_{\emptyset, a_{-}, a_{+}}^{*}(H)
$$

as in Definition 3.3 where we restrict ourselves to admissible Hamiltonians and almost complex structures. We can also define continuation maps and action maps in the same way.

Remark 5.14. Such a definition, along with the theorems, propositions and lemmas used to construct such a definition, are identical except that $M$ is replaced by $M \times T^{*} \mathbb{T}$ and all Hamiltonians and almost complex structures involved are admissible. The only additional ingredient needed is that one needs a maximum principle to prove compactness to ensure that all Floer trajectories stay inside a fixed compact subset of $M \times T^{*} \mathbb{T}$ (see, for example [Oan04, Lemma 1.5]). Also note that since the natural projection map $M \times T^{*} \mathbb{T} \longrightarrow M$ induces an isomorphism $H_{2}\left(M \times T^{*} \mathbb{T} ; \mathbb{Z}\right) \stackrel{\cong}{\longrightarrow} H_{2}(M ; \mathbb{Z})$, we have that $\Lambda_{\mathbb{K}}^{Q_{\omega}}$ and $\Lambda_{\mathbb{K}}^{Q_{\omega},+}$ are the correct Novikov rings to use (and not some larger Novikov rings).

From now on we identify $H_{2}\left(M \times T^{*} \mathbb{T} ; \mathbb{Z}\right)$ with $H_{2}(M ; \mathbb{Z})$ as in the remark above.

Definition 5.15. A closed subset $K \subset \mathbb{T} \times M \times T^{*} \mathbb{T}$ is admissible if there is a compact set $\kappa \subset \mathbb{T} \times M \times T^{*} \mathbb{T}$ and a closed subset $K_{M} \subset M$ so that $K \cup \kappa=\left(\mathbb{T} \times K_{M} \times T^{*} \mathbb{T}\right) \cup \kappa$. In other words, this closed subset is a product $\mathbb{T} \times K_{M} \times T^{*} \mathbb{T}$ near infinity (see Figure 7).

Definition 5.16. For any admissible closed subset $K \subset \mathbb{T} \times M \times T^{*} \mathbb{T}$, we define $\mathcal{H}^{\mathbb{T}, \text { l.s. }}(K, \leq 0)$ to be the set of admissible lower semi-continuous Hamiltonians $H=\left(H_{t}\right)_{t \in \mathbb{T}}$ on $M \times T^{*} \mathbb{T}$ satisfying $H_{t}(x,(\sigma, \tau)) \leq 0$ for $(t, x,(\sigma, \tau)) \in K$ and $H_{t}(x,(\sigma, \tau))=\infty$ for $(t, x,(\sigma, \tau)) \notin K$. A closed subset $K^{\prime} \subset M \times T^{*} \mathbb{T}$ is admissible if $\mathbb{T} \times K^{\prime}$ is admissible. For such a $K^{\prime}$, define $\mathcal{H}^{\mathbb{T}, \text { l.s. }}\left(K^{\prime}, \leq 0\right):=\mathcal{H}^{\mathbb{T}, \text { l.s. }}\left(\mathbb{T} \times K^{\prime}, \leq 0\right)$. 


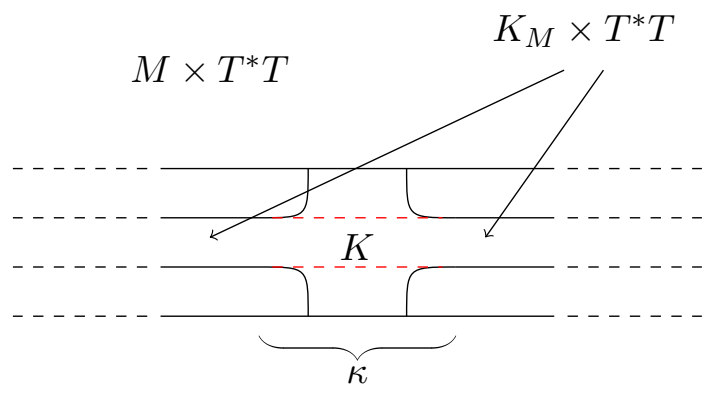

Figure 7. Admissible closed subset.

For any admissible closed subset $K \subset \mathbb{T} \times M \times T^{*} \mathbb{T}$ and any $H \in \mathcal{H}^{\mathbb{T}, \text { l.s. }}(K, \leq 0)$ we define $\mathbb{S} H_{\emptyset, Q_{\omega}, Q_{\omega}}^{*}(H)$ to be the double system

$$
\left(H F_{\emptyset, a_{-}, a_{+}}^{*}(H)\right)_{a_{-}, a_{+} \in \operatorname{Sc}\left(Q_{\omega}\right)} .
$$

If $K_{-}, K_{+} \subset \mathbb{T} \times M \times T^{*} \mathbb{T}$ are admissible closed subsets satisfying $K_{+} \subset K_{-}$and $H^{ \pm} \in$ $\mathcal{H}^{\mathbb{T}, 1 . s .}\left(K_{ \pm}, \leq 0\right)$ satisfies $H^{-} \leq H^{+}$, then the natural morphism of double systems

$$
\mathbb{S} H_{\emptyset, Q_{\omega}, Q_{\omega}}^{*}\left(H^{-}\right) \longrightarrow \mathbb{S} H_{\emptyset, Q_{\omega}, Q_{\omega}}^{*}\left(H^{+}\right)
$$

induced by continuation maps is called a transfer morphism.

We will need the following lemma whose proof is identical to the proof of Lemma 3.6 in the case when $\check{C}=\emptyset$ and $Q=Q_{\omega}$, except that all Hamiltonians and almost complex structures are admissible on $M \times T^{*} \mathbb{T}$.

Lemma 5.17. Let $H=\left(H_{s, t}\right)_{(s, t) \in[0,1] \times \mathbb{T}}$ be a smooth family of autonomous admissible Hamiltonians on $M \times T^{*} \mathbb{T}$ and define $H_{s, \bullet}:=\left(H_{s, t}\right)_{t \in \mathbb{T}}$ for all $s \in[0,1]$. Fix $p \in \mathbb{Z}$ and $a_{ \pm} \in \operatorname{Sc}\left(Q_{\omega}\right)$. Suppose

- $H_{s_{1}, \bullet} \leq H_{s_{2}, \bullet}$ for all $s_{1} \leq s_{2}$ and

- that there are neighborhoods $N_{-}, N_{+}$of $a_{-}, a_{+}$in $\operatorname{Sc}\left(Q_{\omega}\right)$ respectively so that

$$
\Gamma_{\emptyset, a_{-}, a_{+}}^{P}\left(H_{s, \bullet}\right)=\Gamma_{\emptyset, a_{-}^{\prime}, a_{+}^{\prime}}^{P}\left(H_{s, \bullet}\right)
$$

(Definition 2.58) for all $a_{ \pm}^{\prime} \in N_{ \pm}$where $P=[p-1-n, p+1+n]$ for all $s \in \mathbb{R}$.

Then the continuation map

$$
\Phi_{H_{0, \bullet}, H_{1, \bullet}}^{p}: H F_{\check{C}, a_{-}, a_{+}}^{p}\left(H_{0, \bullet}\right) \longrightarrow H F_{\check{C}, a_{-}, a_{+}}^{p}\left(H_{1, \bullet}\right)
$$

in degree $p$ is an isomorphism.

We also have the following proposition whose proof is identical to the proof of Proposition 5.5, except that all Hamiltonians, almost complex structures and closed subsets are admissible on $M \times T^{*} \mathbb{T}$.

Proposition 5.18. Let $K \subset \mathbb{T} \times M \times T^{*} \mathbb{T}$ be an admissible closed subset. Let $H^{-}, H^{+} \in$ $\mathcal{H}^{\mathbb{T}, \text { l.s. }}(K, \leq 0)$ satisfy $H^{-} \leq H^{+}$. Then the transfer morphism

$$
\mathbb{S} H_{\emptyset, Q_{\omega}, Q_{\omega}}^{*}\left(H^{-}\right) \longrightarrow \mathbb{S} H_{\emptyset, Q_{\omega}, Q_{\omega}}^{*}\left(H^{+}\right)
$$

is an isomorphism in 2 -sys- $\Lambda_{\mathbb{K}}^{Q_{\omega},+}$. 
Lemma 5.19. Let $K_{M} \subset M$ be closed and define $K:=K_{M} \times T^{*} \mathbb{T} \subset M \times T^{*} \mathbb{T}$ and let $H \in \mathcal{H}^{\mathbb{T}, 1 . s .}(K, \leq 0)$. Then there is an isomorphism of double systems

$$
\Psi_{K}: \mathbb{S} H_{\emptyset, Q_{\omega}, Q_{\omega}}^{*}(H) \longrightarrow \mathbb{S} H_{\emptyset, Q_{\omega}, Q_{\omega}}^{*}\left(K_{M} \subset M\right) \otimes_{\mathbb{K}} H^{*}(\mathbb{T} ; \mathbb{K})
$$

where $H^{*}(\mathbb{T} ; \mathbb{K})$ is thought of as a double system $\left(H^{*}(\mathbb{T} ; \mathbb{K})\right)_{(i, k) \in\{\star\} \times\{\star\}}$ where $\{\star\}$ is the single element (inverse) directed set. Such a morphism commutes with transfer maps. In other words, for any admissible closed subsets $K^{+}=K_{M}^{+} \times T^{*} \mathbb{T} \subset K^{-}=K_{M}^{-} \times T^{*} \mathbb{T} \subset M \times T^{*} \mathbb{T}$ and any $H^{ \pm} \in \mathcal{H}^{\mathbb{T}, \text { l.s. }}\left(K^{ \pm}, \leq 0\right)$ satisfying $H^{-} \leq H^{+}$, we have the following commutative diagram in 2 -sys- $\Lambda_{\mathbb{K}}^{Q_{\omega},+}$ :

$$
\begin{aligned}
& \mathbb{S} H_{\emptyset, Q_{\omega}, Q_{\omega}}^{*}\left(H^{-}\right) \stackrel{\Psi_{K^{-}}^{\longrightarrow}}{\longrightarrow} \mathbb{S} H_{\emptyset, Q_{\omega}, Q_{\omega}}^{*}\left(K_{M}^{-} \subset M\right) \otimes_{\mathbb{K}} H^{*}(\mathbb{T} ; \mathbb{K}) \\
& \mathbb{S} H_{\emptyset, Q_{\omega}, Q_{\omega}}^{*}\left(H^{+}\right) \stackrel{\Psi_{K^{+}}}{\longrightarrow} \mathbb{S} H_{\emptyset, Q_{\omega}, Q_{\omega}}^{*}\left(K_{M}^{+} \subset M\right) \otimes_{\mathbb{K}} H^{*}(\mathbb{T} ; \mathbb{K})
\end{aligned}
$$

where the vertical morphisms are induced by transfer morphisms.

Proof of Lemma 5.19. Let $f: \mathbb{R} \longrightarrow \mathbb{R}$ be a smooth function so that

- $f(0)=0, f^{\prime \prime}(0)<0$,

- $f^{\prime}(x)>0$ for $x<0$ and $f^{\prime}(x)<0$ for $x>0$ and

- $f(x)=-\frac{1}{2}|x|$ for $|x| \geq 1$ (See Figure 8).

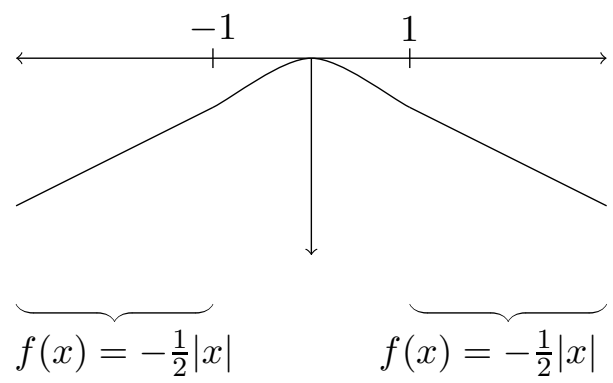

Figure 8. Graph of $f$.

Define

$$
H_{K_{M}}: M \longrightarrow \mathbb{R}, \quad H_{K_{M}}(x)= \begin{cases}0 & \text { if } x \in K_{M} \\ \infty & \text { otherwise }\end{cases}
$$

and let $\pi_{M}: M \times T^{*} \mathbb{T} \longrightarrow M$ be the natural projection map. Then by combining [CFHW96, Proposition 2.2] with a Künneth formula argument, we get an isomorphism

$$
H F_{\emptyset, a_{-}, a_{+}}^{*}\left(\pi_{M}^{*}\left(H_{K_{M}}\right)+f(\sigma)\right) \stackrel{\cong}{\longrightarrow} H F_{\emptyset, a_{-}, a_{+}}^{*}\left(H_{K_{M}}\right) \otimes_{\mathbb{K}} H^{*}(\mathbb{T} ; \mathbb{K})
$$

for all $a_{-}, a_{+} \in \operatorname{Sc}\left(Q_{\omega}, Q_{\omega}\right)$ since the only capped 1-periodic orbits of $f(\sigma): T^{*} \mathbb{T} \longrightarrow \mathbb{R}$ are the constant orbits at its maximum. Such an isomorphism commutes with action maps and continuation maps (possibly tensored with $H^{*}(\mathbb{T} ; \mathbb{K})$ ). Hence we get our isomorphism (5.10) when $H=\pi_{M}^{*}\left(H_{K_{M}}\right)+f(\sigma)$. For general $H$ this isomorphism exists by Proposition 5.18. Again because the isomorphism (5.11) commutes with continuation maps, we get that the isomorphism (5.10) commutes with transfer morphisms. 
Lemma 5.20. Let $K_{M} \subset M$ be a closed subset and let $\nu>0$. Let $H^{ \pm} \in \mathcal{H}^{\mathbb{T}, \text { l.s. }}\left(K^{ \pm}, \leq 0\right)$ where

$$
K_{-}:=\left(K_{M} \times T^{*} \mathbb{T}\right) \cup(M \times\{\sigma \geq \nu\}), \quad K_{+}:=K_{M} \times T^{*} \mathbb{T}
$$

(See Figure 9) and where $H^{-} \leq H^{+}$. Then the transfer morphism

$$
\mathbb{S} H_{\breve{C}, Q_{\omega}, Q_{\omega}}^{*}\left(H^{-}\right) \longrightarrow \mathbb{S} H_{\check{C}, Q_{\omega}, Q_{\omega}}^{*}\left(H^{+}\right)
$$

is an isomorphism in 2 -sys- $\Lambda_{\mathbb{K}}^{Q \omega,+}$.

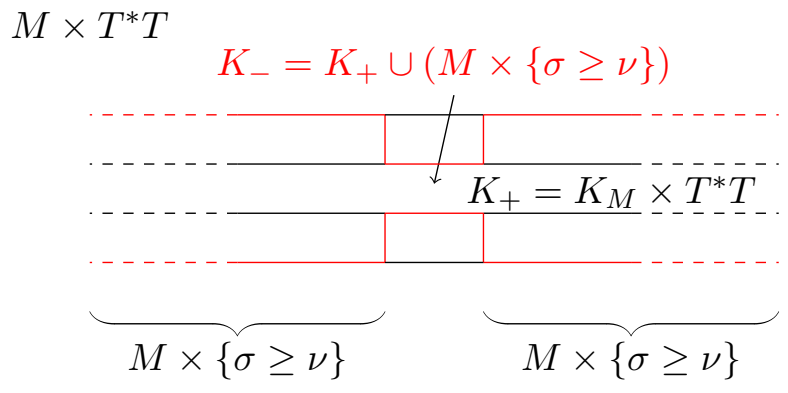

Figure 9. The subsets $K_{-}$and $K_{+}$.

Proof of Lemma 5.20. Let $\pi: M \times T^{*} \mathbb{T} \longrightarrow M$ be the natural projection map. Let $\left(K^{k}\right)_{k \in \mathbb{N}}$ be a sequence of smooth functions on $M$ so that

(1) $K^{k} \leq K^{k+1}$ for all $k \in \mathbb{N}$,

(2) $\left.K^{k}\right|_{M-K_{M}}>0$ and $\left.K^{k}\right|_{K_{M}}=0$ and

(3) for each $x \in M-K_{M}, K^{k}(x)$ tends to infinity as $k$ tends to infinity.

Let $\rho: \mathbb{R} \longrightarrow \mathbb{R}$ be a smooth function so that

(1) $\left.\rho\right|_{[-\nu / 2, \nu / 2]}=1$,

(2) $\left.\rho\right|_{(-\nu, \nu)}>0,\left.\rho\right|_{(-\infty,-\nu] \cup[\nu, \infty)}=0$ and

(3) $\left.\rho^{\prime}\right|_{(-\nu,-\nu / 2)}>0,\left.\rho^{\prime}\right|_{(\nu / 2, \nu)}<0$ (See Figure 10).

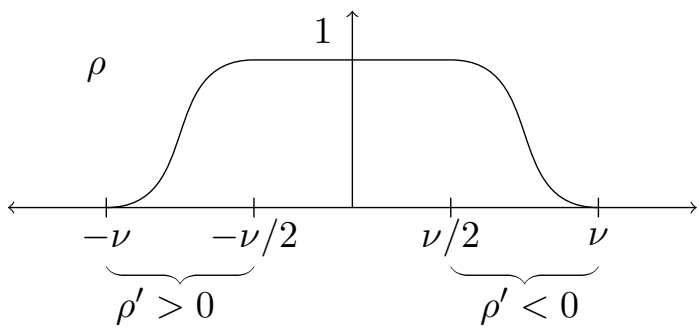

Figure 10. Graph of $\rho$.

Let $f: \mathbb{R} \longrightarrow \mathbb{R}$ be a smooth function so that

(1) $f(0)=0$,

(2) $\left.f^{\prime}\right|_{(-\infty, 0)}>0,\left.f^{\prime}\right|_{(0, \infty)}<0$ and 
(3) $f(x)=-\frac{1}{2}|x|$ for $|x| \geq \nu$ (See Figure 11).

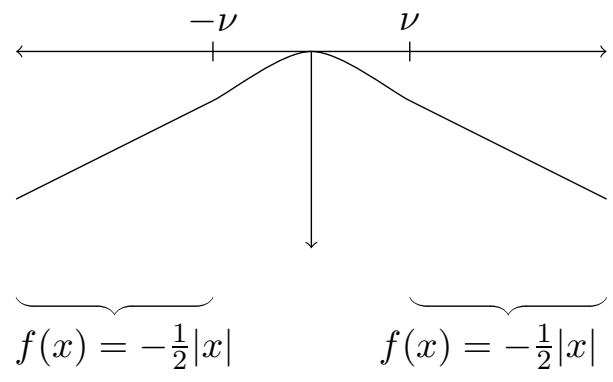

FiguRE 11. Graph of $f$.

Define

$$
H^{k, s}: M \times T^{*} \mathbb{T} \longrightarrow \mathbb{R}, \quad H^{k, s}(x,(\sigma, \tau)):=(s+(1-s) \rho(\sigma)) K^{k}(x)+f(\sigma)-\frac{1}{k}
$$

for each $k \in \mathbb{N}$ and $s \in[0,1]$. Define $H^{k,-}:=H^{k, 0}$ and $H^{k,+}:=H^{k, 1}$. Also define

$$
\check{H}^{ \pm}: M \times T^{*} \mathbb{T} \longrightarrow \mathbb{R}, \quad \check{H}^{ \pm}(x,(\sigma, \tau)):=\left\{\begin{array}{ll}
\infty & \text { if }(x,(\sigma, \tau)) \notin K_{ \pm} \\
f(\sigma) & \text { otherwise. }
\end{array}\right\} .
$$

By Lemma 8.5, we can choose a sequence of elements $\left(a_{i}\right)_{i \in \mathbb{Z}}$ in $\operatorname{Sc}\left(Q_{\omega}\right)$ so that

- $a_{i}([\omega], 1,1)$ is not in the action spectrum of $H^{k, \pm}$ for all $k, j \in \mathbb{N}$ and

- $a_{i}$ tends to infinity as $i$ tends to infinity and $a_{i}$ tends to $-\infty$ as $i$ tends to $-\infty$.

Then

$$
H F_{\left(a_{i}, a_{j}\right)}^{*}\left(\check{H}^{ \pm}\right)=\underset{k \in \mathbb{N}}{\lim }\left(H F_{\left(a_{i}, a_{j}\right)}^{*}\left(H^{k, \pm}\right)\right)
$$

for each $i, j \in \mathbb{Z}$ and such an isomorphism is induced by continuation maps. Hence by Lemma 2.40 , we have an inclusion isomorphism of double systems

$$
\left(H F_{\left(a_{i}, a_{j}\right)}^{*}\left(\check{H}^{ \pm}\right)\right)_{(i, j) \in \mathbb{Z} \times \mathbb{Z}} \stackrel{\cong}{\longrightarrow} \mathbb{S} H_{\emptyset, Q_{\omega}, Q_{\omega}}^{*}\left(\check{H}^{k, \pm}\right)
$$

Since all the null homologous 1-periodic orbits of $H^{k, s}$ are contained in $\{\sigma=0\}$ because $\sigma \frac{\partial H^{k, s}}{\partial \sigma}<0$ and $\frac{\partial H^{k, s}}{\partial \tau}=0$ away from $\{\sigma=0\}$ for each $s \in[0,1]$, we have by Lemma 5.17 that the continuation map

$$
H F_{\left(a_{i}, a_{j}\right)}^{*}\left(H^{k,-}\right) \longrightarrow H F_{\left(a_{i}, a_{j}\right)}^{*}\left(H^{k,+}\right)
$$

is an isomorphism for each $i, j \in \mathbb{Z}$ and $k \in \mathbb{N}$. Combining this with the fact that the inclusion map (5.13) is an isomorphism, we have that the continuation map

$$
\mathbb{S} H_{\emptyset, Q_{\omega}, Q_{\omega}}^{*}\left(\check{H}^{-}\right) \longrightarrow \mathbb{S} H_{\emptyset, Q_{\omega}, Q_{\omega}}^{*}\left(\check{H}^{+}\right)
$$

is an isomorphism. Hence by Proposition 5.18, the map (5.12) is an isomorphism.

Lemma 5.21. Let $K^{ \pm} \subset M \times T^{*} \mathbb{T}$ be admissible closed subsets so that $K^{+} \subset K^{-}$. Let $H^{ \pm} \in \mathcal{H}^{\mathbb{T}, \text {,.s. }}\left(K^{ \pm}, \leq 0\right)$ (Definition 5.16) satisfy $H^{-} \leq H^{+}$and let $\check{H}^{ \pm} \in \mathcal{H}^{\mathbb{T}, \text { l.s. }}\left(\left(\mathbb{T} \times K^{ \pm}\right) \cup\right.$ $\left.\left([0,1 / 2] \times M \times T^{*} \mathbb{T}\right), \leq 0\right)$ satisfy $\check{H}^{-} \leq \check{H}^{+}$. Then the transfer map

$$
\mathbb{S} H_{\breve{C}, Q_{\omega}, Q_{\omega}}^{*}\left(H^{-}\right) \longrightarrow \mathbb{S} H_{\breve{C}, Q_{\omega}, Q_{\omega}}^{*}\left(H^{+}\right)
$$


is an isomorphism if and only if the transfer map

$$
\mathbb{S} H_{\breve{C}, Q_{\omega}, Q_{\omega}}^{*}\left(\check{H}^{-}\right) \longrightarrow \mathbb{S} H_{\breve{C}, Q_{\omega}, Q_{\omega}}^{*}\left(\check{H}^{+}\right)
$$

is an isomorphism.

Proof. By Proposition 5.18, it is sufficient for us to prove this for specific $H^{ \pm}$and $\check{H}^{ \pm}$. Let $F: \mathbb{T} \longrightarrow \mathbb{T}$ be a non-decreasing smooth function homotopic to the identity so that $F^{\prime}(t)>0$ for $t \in(1 / 2,1)$ and $F^{\prime}(t)=0$ for $t \in[0,1 / 2]$. Choose $H^{ \pm}$and $\check{H}^{ \pm}$so that $\left(H^{ \pm}\right)^{F}=\check{H}^{ \pm}$ where $\left(H^{ \pm}\right)^{F}$ is given in Definition 3.9. Then our lemma follows from the fact that we have reparameterization isomorphisms

$$
\mathbb{S} H_{\check{C}, Q_{\omega}, Q_{\omega}}^{*}\left(H^{ \pm}\right) \longrightarrow \mathbb{S} H_{\check{C}, Q_{\omega}, Q_{\omega}}^{*}\left(\check{H}^{ \pm}\right)
$$

which commute with continuation maps.

Proof of Theorem 5.12. The key idea of the proof is to use the displacing Hamiltonian $\mathrm{H}^{-}$ to construct a family of Hamiltonians $\left.\left(H^{-}+H_{s, \bullet}\right)_{s \in[0, \infty)}\right)$ (see below) with identical orbits realizing the transfer isomorphism (5.9). One then uses Lemma 5.17.

For each $\nu>0$, define

$$
\widehat{K}_{\nu}:=\left(K \times T^{*} \mathbb{T}\right) \cup(M \times\{\sigma \geq \nu\})
$$

and

$$
\widetilde{K}_{\nu}:=\left(\mathbb{T} \times \widehat{K}_{\nu}\right) \cup\left([0,1 / 2] \times M \times T^{*} \mathbb{T}\right) .
$$

By Lemmas 5.19, 5.20 and 5.21, it is sufficient for us to show that

$$
\mathbb{S} H_{\breve{C}, Q_{\omega}, Q_{\omega}}^{*}\left(H^{-}\right) \longrightarrow \mathbb{S} H_{\breve{C}, Q_{\omega}, Q_{\omega}}^{*}\left(H^{+}\right)
$$

is an isomorphism for some $\nu>0$ and some appropriate lower-semi-continuous Hamiltonians $H^{-} \in \mathcal{H}^{\mathbb{T}, \text {,.s.s. }}\left(M \times T^{*} \mathbb{T}, \leq 0\right), H^{+} \in \mathcal{H}^{\mathbb{T}, \text { l.s. }}\left(\widetilde{K}_{\nu}, \leq 0\right)$ satisfying $H^{-} \leq H^{+}$.

Since $(\overline{M-K}) \times(\{0\} \times \mathbb{T})$ is Hamiltonian displaceable inside $M \times T^{*} \mathbb{T}$, there is a smooth admissible Hamiltonian $\check{H}=\left(\check{H}_{t}\right)_{t \in \mathbb{T}}$ on $M \times T^{*} \mathbb{T}$ and a constant $\nu>0$ so that $\phi_{1}^{\check{H}}(Q) \cap Q=\emptyset$ where

$$
Q:=M \times T^{*} \mathbb{T}-\widehat{K}_{\nu}=(M-K) \times\{\sigma<\nu\} .
$$

By subtracting a constant from $\check{H}$, we can assume that $\check{H}<0$. Now let $F, G: \mathbb{T} \longrightarrow \mathbb{T}$ be a smooth non-decreasing functions homotopic to the identity map satisfying $F(0)=0$, $\left.F^{\prime}\right|_{[0,1 / 2]}=0,\left.F^{\prime}\right|_{(1 / 2,1)}>0$ and $G(0)=0,\left.G^{\prime}\right|_{[1 / 2,1]}=0$ and $\left.G^{\prime}\right|_{(0,1 / 2)}>0$. Define $H^{-}:=\check{H}^{G}$. We define the lower semi-continuous Hamiltonian $H^{+}:=\left(H_{t}^{+}\right)_{t \in \mathbb{T}}$ by

$$
H_{t}^{+}(x):= \begin{cases}H_{t}^{-}(x) & \text { if }(t, x) \in \widetilde{K}_{\nu} \\ \infty & \text { otherwise. }\end{cases}
$$

Now choose a smooth family of autonomous Hamiltonians $H=\left(H_{s, t}\right)_{(s, t) \in[0, \infty) \times \mathbb{T}}$ on $M \times$ $T^{*} \mathbb{T}$ satisfying

- $H_{s, t} \geq 0, \frac{\partial H_{s, t}}{\partial s} \geq 0$

- $H_{s, t}(x)=0$ if and only if $(t, x) \in \widetilde{K}_{\nu}$ or $s=0$,

- $\left.d H_{s, t}\right|_{x}=0$ for all $(t, x) \in \widetilde{K}_{\nu}$.

- $H_{s, t}(x) \rightarrow \infty$ as $s \rightarrow \infty$ for all $(t, x) \in \mathbb{T} \times M \times T^{*} \mathbb{T}-\widetilde{K}_{\nu}$.

Define $H_{s, \bullet}:=\left(H_{s, t}\right)_{t \in \mathbb{T}}$ for all $s \in[0, \infty)$. By Lemma 8.5, there is a sequence $\left(a_{i}\right)_{i \in \mathbb{Z}}$ in $\operatorname{Sc}\left(Q_{\omega}\right)$ so that

- $a_{i}([\omega], 1,1) \rightarrow \pm \infty$ as $i \rightarrow \pm \infty$ and

- $a_{i}([\omega], 1,1)$ is not in the action spectrum of $H^{-}$for each $i \in \mathbb{Z}$. 
Note that the capped 1-periodic orbits of $H^{-}$and $H_{s, \bullet}+H^{-}$are identical for all $s \in[0, \infty)$ since the support of $H_{s, t}$ is contained inside $(1 / 2,1) \times Q$ for all $(s, t) \in[0, \infty) \times \mathbb{T}$ (making $H_{s, \bullet}$ and $H^{-}$Poisson commute) and since there are no 1-periodic orbits $\gamma: \mathbb{T} \longrightarrow M \times T^{*} \mathbb{T}$ of $H^{-}$or $H_{s, \bullet}+H^{-}$satisfying $\gamma(0) \in Q$ for each $s \in[0, \infty)$. Also, the corresponding actions of these capped 1-periodic orbits are the same as well since $\left.H_{t}^{-}\right|_{\gamma(t)}=\left.\left(H_{s, \bullet}+H^{-}\right)_{t}\right|_{\gamma(t)}$ for all $t \in \mathbb{T}$ and all 1-periodic orbits $\gamma$ of $H^{-}$. Therefore by Lemma 5.17, the natural continuation map

$$
H F_{\left(a_{i}, a_{j}\right)}^{*}\left(H^{-}\right) \longrightarrow H F_{\left(a_{i}, a_{j}\right)}^{*}\left(H_{s, \bullet}+H^{-}\right)
$$

is an isomorphism for all $i, j \in \mathbb{Z}$ and $s \in[0, \infty)$. Hence the induced map of double systems

$$
\left(H F_{\left(a_{i}, a_{j}\right)}^{*}\left(H^{-}\right)\right)_{(i, j) \in \mathbb{Z}} \longrightarrow\left(H F_{\left(a_{i}, a_{j}\right)}^{*}\left(H_{s, \bullet}+H^{-}\right)\right)_{(i, j) \in \mathbb{Z}}
$$

is an isomorphism. By Lemma 2.40, this implies that

$$
\mathbb{S} H_{\breve{C}, Q_{\omega}, Q_{\omega}}^{*}\left(H^{-}\right) \longrightarrow \mathbb{S} H_{\breve{C}, Q_{\omega}, Q_{\omega}}^{*}\left(H^{+}\right)
$$

is an isomorphism. This completes our proposition.

5.5. Symplectic Cohomology and Alternative Filtrations. In this subsection we will show in Proposition 5.24 below that symplectic cohomology defined with respect to a particular wide action interval domain (Definition 2.58) is isomorphic to one defined over an action interval domain that is not wide under certain conditions.

Definition 5.22. Let $\left(C, \alpha_{C}\right)$ be a $2 n-1$-manifold with contact form. Recall that the Reeb vector field of $\alpha_{C}$ is the unique vector field $R_{\alpha_{C}}$ satisfying $i_{R_{\alpha_{C}}} d \alpha_{C}=0$ and $\alpha_{C}\left(i_{R_{\alpha_{C}}}\right)=1$. A periodic Reeb orbit of length $\lambda>0$ is a map $\gamma: \mathbb{R} / \lambda \mathbb{Z} \longrightarrow C$ satisfying $\dot{\gamma}=R_{\alpha_{C}}$. The Reeb flow of $\alpha_{C}$ is the flow $\left(\phi_{t}^{\alpha}: C \longrightarrow C\right)_{t \in \mathbb{R}}$ of $R_{\alpha_{C}}$. We will define length $(\gamma):=\lambda$.

Now let $\breve{C}=[1-\epsilon, 1+\epsilon] \times C \subset M$ be a contact cylinder inside $M$ and let $\iota_{C}: C \longrightarrow M$ be the natural inclusion map sending $x$ to $(1, x) \in \check{C} \subset M$. Let $r_{C}$ be the cylindrical coordinate of $\check{C}$ and let $\alpha_{C}$ be the associated contact form. By abuse of notation, we will define $R_{\alpha_{C}}$ to be the unique vector field on $\check{C}$ which projects to $R_{\alpha_{C}}$ in $C$ and 0 in $[1-\epsilon, 1+\epsilon]$. We let $\frac{\partial}{\partial r_{C}}$ be the gradient of $r_{C}$ with respect to any product metric on $\check{C}$ where the factor $[1-\epsilon, 1+\epsilon]$ has the standard Euclidean metric. Let $\gamma$ be a periodic Reeb orbit of $\alpha_{C}$ of length $\lambda$ so that $\iota_{C} \circ \gamma$ is null homologous in $M$. Let $\widehat{\gamma}=(\widetilde{\gamma}, \check{\gamma})$ be a capped loop so that $\gamma(\lambda t)=\widetilde{\gamma}(\check{\gamma}(t))$ for each $t \in$ $[0,1]$. Then since we have a splitting $\left.T M\right|_{\check{C}}=\operatorname{ker}\left(\alpha_{C}\right) \oplus \operatorname{Span}\left(\frac{\partial}{\partial r_{C}}, R_{\alpha_{C}}\right)$ of symplectic vector bundles with associated symplectic forms $\left.d \alpha_{C}\right|_{\operatorname{ker}\left(\alpha_{C}\right)}$ and $\left.d r_{C} \wedge \alpha_{C}\right|_{\operatorname{Span}\left(\frac{\partial}{\partial r_{C}}, R_{\alpha_{C}}\right)}$ and since $\operatorname{Span}\left(\frac{\partial}{\partial r_{C}}, R_{\alpha_{C}}\right)$ has a natural choice of symplectic trivialization, any symplectic trivialization of $\widetilde{\gamma}^{*} T M$ gives an induced symplectic bundle trivialization

$$
\tau: \gamma^{*} \operatorname{ker}\left(\alpha_{C}\right) \longrightarrow(\mathbb{R} / \lambda \mathbb{Z}) \times \mathbb{C}^{n-1} .
$$

Let $P:(\mathbb{R} / \lambda \mathbb{Z}) \times \mathbb{C}^{n-1} \longrightarrow \mathbb{C}^{n-1}$ be the natural projection map. The Conley-Zehnder index $C Z(\gamma)$ of $\gamma$ is defined to be the Conley-Zehnder index of the path of symplectic matrices

$$
P \circ \tau \circ \phi_{t}^{\alpha} \circ\left(\left.P \circ \tau\right|_{0}\right)^{-1}, \quad t \in[0, \lambda] .
$$

This does not depend on the choice of trivialization $\tau$ by (CZ4) or on the choice of $\widehat{\gamma}$ since $c_{1}(M)=0$. The index of a Reeb orbit $\gamma$ is defined to be $|\gamma|:=n-C Z(\gamma)$.

Let $\Gamma_{\alpha_{C}}$ be the set of periodic Reeb orbits $\gamma$ of $\alpha_{C}$ so that $\iota_{C} \circ \gamma$ is null homologous inside $M$. The index $[-m, m]$ period spectrum of $\check{C}$ is the set

$$
\left\{\operatorname{length}(\gamma): \gamma \in \Gamma_{\alpha_{C}},-m \leq|\gamma| \leq m\right\} \subset \mathbb{R} \text {. }
$$


Definition 5.23. The contact cylinder $\check{C}$ as above is index bounded if for every $m>0$, there exists $\mu_{m}>0$ so that the index $[-m, m]$ period spectrum of $\check{C}$ is contained in the interval $\left(0, \mu_{m}\right)$.

Proposition 5.24. Suppose that $\check{C}$ is an index bounded contact cylinder with associated Liouville domain D. Let $\omega_{\breve{C}}$ be a $\check{C}$-compatible 2 -form with scaling constants 0 and 1 and which is equal to $\omega$ outside $D \cup([1,1+\epsilon / 2] \times C)$. Also let $Q_{+}=Q_{\omega_{\check{C}}} \subset H^{2}(M, D ; \mathbb{R}) \times \mathbb{R} \times \mathbb{R}$ be the cone spanned by $\left(\left[\omega_{\check{C}}\right], 1,1\right)$ and $Q_{-}$the cone spanned by $\left(\left[\omega_{\check{C}}\right], 1,1\right)$ and $\left(\left[\omega_{\check{C}}\right], 0,1\right)$. Then the action map

$$
\mathbb{S} H_{\breve{C}, Q_{-}, Q_{+}}^{*}(D \subset M) \longrightarrow \mathbb{S} H_{\breve{C}, Q_{+}, Q_{+}}^{*}(D \subset M)
$$

is an isomorphism in 2 -sys- $\Lambda_{\mathbb{K}}^{Q_{+},+}$.

Before we prove Proposition 5.24, we need some preliminary lemmas. The first lemma relates the indices of Reeb orbits with the indices of certain Hamiltonian orbits.

Lemma 5.25. Let $\check{C}=[1-\epsilon, 1+\epsilon] \times C$ be an index bounded contact cylinder with cylindrical coordinate $r_{C}$ and associated contact form $\alpha_{C}$ and let $\pi: \check{C} \longrightarrow C$ be the natural projection map. Let $f:[1-\epsilon, 1+\epsilon] \longrightarrow \mathbb{R}$ be a smooth function, $R_{\lambda,[-m, m]}$ the set of Reeb orbits in $\Gamma_{\alpha_{C}}$ of length $\lambda$ and index in $[-m, m]$ and $O_{\lambda,[-m, m]}$ the set of 1-periodic orbits of $f\left(r_{C}\right)$ contained in $\left\{r_{C}=\left(f^{\prime}\right)^{-1}(\lambda)\right\}$ of index in $[-m, m]$ which are null homologous in $M$. Then the map

$$
O_{\lambda,[-m, m]} \longrightarrow R_{\lambda,\left[-m-\frac{1}{2}, m+\frac{1}{2}\right]}
$$

sending $\gamma: \mathbb{T} \longrightarrow \check{C}$ to $\pi \circ \gamma \circ b_{\lambda}$ is well defined where

$$
b_{\lambda}:[0, \lambda] \longrightarrow[0,1], \quad b_{\lambda}(t):=(1 / \lambda) t, \quad \forall t \in[0, \lambda] .
$$

Proof. Since $X_{f\left(r_{C}\right)}=f^{\prime}\left(r_{C}\right) R_{\alpha_{C}}$ where $R_{\alpha_{C}}$ is the natural lift of the Reeb vector field of $\alpha_{C}$ to $\check{C}$, we see that $\gamma \in O_{\lambda,[m, m]}$ gets pushed forward to a Reeb orbit $\gamma \circ \pi \circ b_{\lambda}$ of length $\lambda$. Therefore, all we need to do is compute the index of $\gamma \circ \pi \circ b_{\lambda}$.

Let $\xi:=\operatorname{ker}\left(\pi^{*} \alpha_{C}\right) \cap \operatorname{ker}\left(d r_{C}\right)$ be a codimension 2 distribution inside $\check{C}$ and define $\xi^{\perp}:=$ $\operatorname{Span}\left(\frac{\partial}{\partial r_{C}}, R_{\alpha_{C}}\right)$. Then these are symplectic subbundles of $\gamma^{*} T M$ which are symplectically orthogonal to each other. We have a natural symplectic trivialization $T: \gamma^{*} \xi^{\perp} \longrightarrow \mathbb{T} \times \mathbb{C}$ sending $\frac{\partial}{\partial r}$ to $\frac{\partial}{\partial x}$ and $R$ to $\frac{\partial}{\partial y}$ where $z=x+i y$ is the natural complex coordinate on $\mathbb{C}$.

Let $\widehat{\gamma}:=(\widetilde{\gamma}, \check{\gamma})$ be a capped loop whose associated loop is $\gamma$ and where the domain of $\widetilde{\gamma}$ is an oriented surface $\Sigma$. Let $\iota: \gamma^{*} T M \longrightarrow \widetilde{\gamma}^{*} T M$ be the natural bundle inclusion map covering $\check{\gamma}$. Let

$$
\tau: \widetilde{\gamma}^{*} T M \longrightarrow \Sigma \times \mathbb{C}^{n}, \quad \check{\tau}: \gamma^{*} \xi \longrightarrow \mathbb{T} \times \mathbb{C}^{n-1}
$$

be symplectic bundle trivializations so that $\tau \circ \iota=\check{\tau} \oplus T$ after identifying $\partial \Sigma$ with $\mathbb{T}$ via $\check{\gamma}$. Let $P_{\mathbb{C}^{n}}: \Sigma \times \mathbb{C}^{n} \longrightarrow \mathbb{C}^{n}$ be the natural projection map. Let $\phi_{t}: \check{C} \longrightarrow \check{C}$ be the time $t$ Hamiltonian flow of $f\left(r_{C}\right)$. We have that

$$
P_{\mathbb{C}^{n}} \circ \tau \circ D \phi_{t} \circ\left(\left.P_{\mathbb{C}^{n}} \circ \tau\right|_{\check{\gamma}(0)}\right)^{-1}: \mathbb{C}^{n} \longrightarrow \mathbb{C}^{n}
$$

is equal to a block diagonal matrix

$$
\left(\begin{array}{cc}
A_{t} & 0 \\
0 & B_{t}
\end{array}\right)
$$


with respect to the splitting $\mathbb{C}^{n} \cong \mathbb{C}^{n-1} \oplus \mathbb{C}$ for all $t \in[0,1]$. By Definition 5.22 and (CZ4) we have $C Z\left(\left(A_{t}\right)_{t \in \mathbb{T}}\right)=C Z\left(\pi \circ \gamma \circ b_{\lambda}\right)$. Also $B_{t}=\left(\begin{array}{cc}1 & f^{\prime \prime}(r) t \\ 0 & 1\end{array}\right)$ for all $t \in[0,1]$. Hence

$$
C Z(\gamma) \stackrel{(\mathrm{CZ} 2)}{=} C Z\left(\left(A_{t}\right)_{t \in \mathbb{T}}\right)+C Z\left(\left(B_{t}\right)_{t \in \mathbb{T}}\right) \stackrel{(\mathrm{CZ} 5)}{=} C Z\left(\left(A_{t}\right)\right)+\eta
$$

where $\eta=0$ or $\pm \frac{1}{2}$ and so

$$
\left|C Z(\gamma)-C Z\left(\pi \circ \gamma \circ b_{\lambda}\right)\right| \leq \frac{1}{2}
$$

and this completes our lemma.

The following lemma constructs for us a smooth family of Hamiltonians (which will compute symplectic cohomology later on) with the property that all the 1-periodic orbits in a fixed index range and whose action is not too big are identical.

Lemma 5.26. Let $\check{C}, D$ be as in Proposition 5.24. Let $m>0$ be a constant. Then there is a smooth family of autonomous $\check{C}$-compatible Hamiltonians $H=\left(H_{s}\right)_{s \in[0, \infty)}$ on $M$ so that

(1) $H_{s}$ is locally constant outside $[1-\epsilon / 2,1+\epsilon / 8] \times C$ and a non-decreasing function of the radial coordinate of $\check{C}$ inside $\check{C}$ which has positive derivative near $\partial D$,

(2) $\frac{d H_{s}(x)}{d s} \geq 0$ for all $x \in M$ and $H_{s}(x) \rightarrow \infty$ as $s \rightarrow \infty$ for all $x \in M-D$,

(3) $\left.H_{s}\right|_{D}=\left.H_{0}\right|_{D}<0$ for all $s \in[0, \infty)$ and

(4) for each $\check{C}$-action interval $\left(a_{-}, a_{+}\right) \in \operatorname{Sc}\left(Q_{-}\right) \times \operatorname{Sc}\left(Q_{+}\right)$where $\left(Q_{-}, Q_{+}\right)$is wide, there exists $S \geq 0$ (depending continuously on $\left(a_{-}, a_{+}\right)$) so that for each $s \geq S$ and each capped 1-periodic orbit $(\widetilde{\gamma}, \check{\gamma}) \in \Gamma_{\check{C}, a_{-}, a_{+}}^{[-m, m]}\left(H_{s}\right)$, we have Image $(\widetilde{\gamma} \circ \check{\gamma}) \subset D$ where $\Gamma_{\breve{C}, a_{-}, a_{+}}^{[-m, m]}\left(H_{s}\right)$ is given in Definition 2.58 .

Remark 5.27. Note that every capped 1-periodic orbit of $H_{s}$ whose associated 1-periodic orbit has image in $D$ is also a capped 1-periodic orbit of $H_{0}$ with the same $\check{C}$ action.

Remark 5.28. We can in fact strengthen Lemma 5.26 slightly. We can show that there is a constant $\delta>0$ (independent of $s \in[0, \infty]$ ) so that properties (1)- (4) from Lemma 5.26 hold with $H_{s}$ replaced by $e^{\tau} H_{s}$ for all $-\delta \leq \tau \leq \delta$. The proof of this stronger lemma is exactly the same as the proof of Lemma 5.26 below, except that the action estimates (5.16) and (5.17) below have to be scaled appropriately. This strengthening will be needed in Lemma 5.33 below.

Proof of Lemma 5.26. Let $\check{C}=[1-\epsilon, 1+\epsilon] \times C$ be our contact cylinder with cylindrical coordinate $r_{C}$ and contact form $\alpha_{C}$. Since $\check{C}$ is index bounded, there exists a constant $\Xi>0$ so that every Reeb orbit of length greater than $\Xi$ has index in $\mathbb{Z}-[-m-2, m+2]$.

Let $f_{s}:[1-\epsilon, 1+\epsilon] \longrightarrow \mathbb{R}, s \in[0, \infty)$ be a smooth family of functions satisfying the following properties (see picture below):

(a) $\frac{d f_{s}(x)}{d s} \geq 0$ for all $x \in M$ and $f_{s}(x)$ tends to infinity as $s \rightarrow \infty$ for each $x>1$,

(b) $f_{s}(x)=f_{0}(x)<0$ for all $x \leq 1$ and there is constant $C_{s} \geq 0$ so that $f_{s}(x)=f_{0}(x)+C_{s}$ for all $x \in[1+\epsilon / 16, \infty)$ and $s \in[0, \infty)$,

(c) $\left.f_{s}\right|_{(-\infty, 1-\epsilon / 2] \cup[1+\epsilon / 8, \infty)}$ is locally constant and

(d) $f_{s}^{\prime} \geq 0$ and $f_{s}^{\prime}(x)>\Xi$ for all $x \in[1,1+\epsilon / 16]$ for all $s \geq 0$ (See Figure 12). 


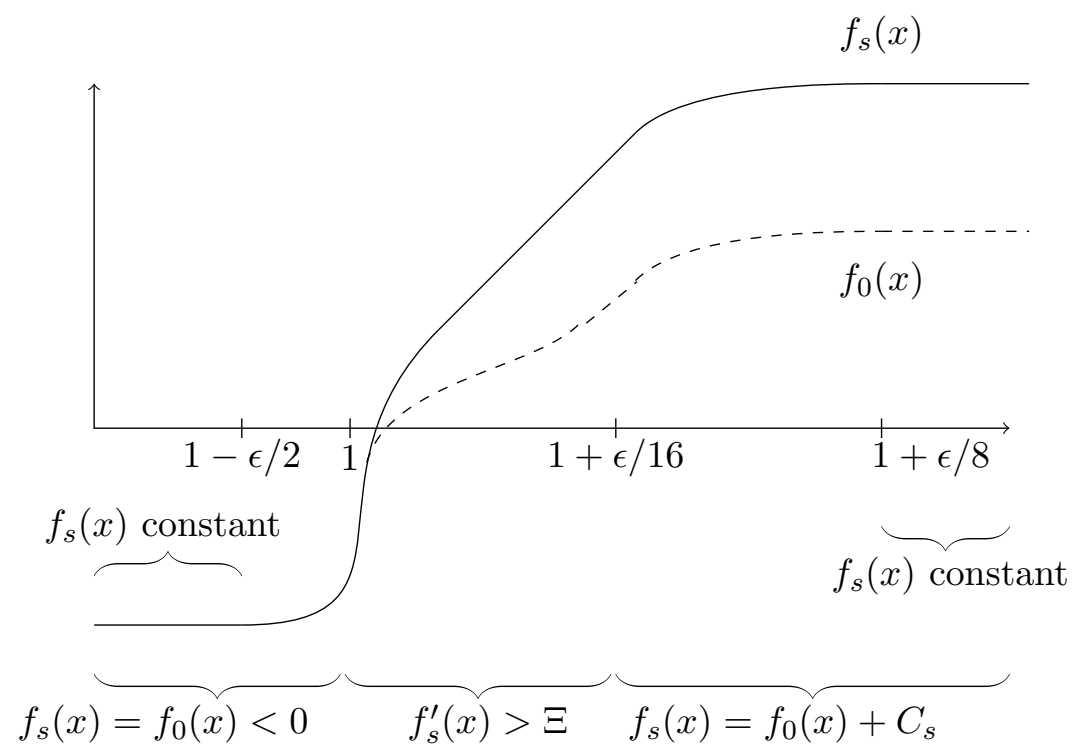

Figure 12. Graph of $f_{s}$ for each $s \in[0, \infty)$.

Define

$$
H_{s}: M \longrightarrow \mathbb{R}, \quad H_{s}(x):= \begin{cases}f_{0}(1-\epsilon / 2) & \text { if } x \in D-\check{C} \\ f_{s}\left(r_{C}\right) & \text { if } x \in \check{C} \\ f_{s}(1+\epsilon / 8) & \text { otherwise }\end{cases}
$$

for each $s \geq 0$. It is clear that $\left(H_{s}\right)_{s \in[0, \infty)}$ satisfies properties (1)-(3). Therefore we only need to show that it satisfies (4). Let $\pi_{D}: H^{2}(M, D ; \mathbb{R}) \times \mathbb{R}^{2} \longrightarrow H^{2}(M, D ; \mathbb{R})$ be the natural projection map. Let $\left(a_{-}, a_{+}\right) \in \operatorname{Sc}\left(Q_{-}, Q_{+}\right)$be a $\check{C}$-action interval where $\left(Q_{-}, Q_{+}\right)$is wide. Let $h:=\operatorname{height}\left(a_{-}, a_{+}\right)$as in Equation (2.15).

Choose a 1 -form $\theta \in \Omega^{1}(M)$ so that $d \theta=\omega-\omega_{\check{C}}$ where $\omega_{\check{C}}$ is a $\check{C}$-compatible 2 -form with scaling constants 0 and 1 and which is equal to $\omega$ outside $D \cup([1,1+\epsilon / 2] \times C)$ and so that $\theta=0$ outside $D \cup([1,1+\epsilon / 2] \times C)$. Since $C_{s}$ tends to infinity as $s \rightarrow \infty$, we can choose $S \geq 0$ so that

$$
\min \left(f_{0}\right)+C_{S}-\sup _{\breve{\gamma}} \int_{\mathbb{T}} \breve{\gamma}^{*} \theta>h
$$

where the infimum is taken over all 1-periodic orbits $\breve{\gamma}: \mathbb{T} \longrightarrow M$ of $H_{0}$. Now if $s \geq S$ and $\gamma=(\widetilde{\gamma}, \check{\gamma}) \in \Gamma_{\check{C}, a_{-}, a_{+}}^{[-m, m]}\left(H_{s}\right)$ is a capped 1-periodic orbit, then by Lemma 5.25 , we have that the image of the 1-periodic orbit $\widetilde{\gamma} \circ \check{\gamma}$ does not intersect $[1,1+\epsilon / 16] \times C$ since $f_{s}^{\prime}(x)>\Xi$ for all $x \in[1,1+\epsilon / 16]$. Suppose, for a contradiction, the image of $\widetilde{\gamma} \circ \check{\gamma}$ does not intersect $D \cup([1,1+\epsilon / 16] \times C)$ then by Equation (2.6),

$$
\begin{gathered}
\left|\mathcal{A}_{H_{s}, \breve{C}}(\gamma)\left(q, \lambda_{+,-}^{q}, \lambda_{+,+}^{q}\right)-\mathcal{A}_{H_{s}, \breve{C}}(\gamma)\left(q, \lambda_{-,-}^{q}, \lambda_{-,+}^{q}\right)\right| \geq \\
\left(\lambda_{+,-}^{q}-\lambda_{-,-}^{q}\right)\left(\min \left(f_{0}\right)+C_{s}-\int_{0}^{1} \check{\gamma}^{*} \theta\right)
\end{gathered}
$$

for every $\left(q, \lambda_{ \pm,-}^{q}, \lambda_{ \pm,+}^{q}\right) \in Q_{ \pm}$satisfying $\lambda_{-,-}^{q}<\lambda_{+,-}^{q}$ and $\lambda_{+,+}^{q}=\lambda_{-,+}^{q}$. But this contradicts Equation (5.16) and the fact that $\gamma \in \Gamma_{\check{C}, a_{-}, a_{+}}^{[-m, m]}\left(H_{s}\right)$. Hence $\widetilde{\gamma} \circ \check{\gamma}$ must have image contained in $D$. 
Proof of Proposition 5.24. In this proof we will use the family of Hamiltonians from Lemma 5.26 to compute symplectic cohomology and show that our action map (5.15) is an isomorphism. The key idea of the proof is to show that the 1-periodic orbits of these Hamiltonians outside $D$ are not needed to compute these symplectic cohomology groups mainly by using property (4) from Lemma 5.15.

Fix $p \in \mathbb{Z}$. Let $\left(H_{s}\right)_{s \in[0, \infty)}$ be as in Lemma 5.26 with $m=|p|+n+1$. Since the 1-periodic orbits of $H_{s}$ form a compact subset of $C^{\infty}(\mathbb{T}, M)$, there is a non-decreasing family of positive constants $\left(c_{s}\right)_{s \in[0, \infty)}$ so that for each $s \geq 0$ and each capped 1-periodic orbit $\gamma$ of $H_{s}$,

$$
\left|\mathcal{A}_{H_{s}, \check{C}}(\gamma)\left(\left[\omega_{\check{C}}\right], 1,1\right)-\mathcal{A}_{H_{s}, \check{C}}(\gamma)\left(\left[\omega_{\check{C}}\right], 0,1\right)\right|<c_{s} .
$$

Let $a_{s}: Q_{-} \longrightarrow \mathbb{R}$ be the unique linear map satisfying $a_{s}\left(\left[\omega_{\check{C}}\right], 1,1\right)=0$ and $a_{s}\left(\left[\omega_{\check{C}}\right], 0,1\right)=$ $-c_{s}$. Then for each $\check{C}$-action interval $\left(a_{-}, a_{+}\right) \in \operatorname{Sc}\left(Q_{-}\right) \times \operatorname{Sc}\left(Q_{+}\right)$, the action morphism

$$
H F_{\check{C}, a_{-}+a_{s}, a_{+}}^{p}\left(H_{s}\right) \longrightarrow H F_{\check{C},\left.a_{-}\right|_{Q_{+}},\left.a_{+}\right|_{Q_{+}}}^{p}\left(H_{s}\right)
$$

is an isomorphism for each $s \geq 0$ by Equation (5.18) combined with Lemma 3.8. Also if

$$
a_{-}\left(\left[\omega_{\check{C}}\right], 0,1\right)<a_{-}\left(\left[\omega_{\check{C}}\right], 1,1\right)-c_{0},
$$

then there is a function $v: \operatorname{Sc}\left(Q_{-}\right) \times \operatorname{Sc}\left(Q_{+}\right) \longrightarrow[0, \infty)$ so that the action morphism

$$
H F_{\check{C}, a_{-}, a_{+}}^{p}\left(H_{\check{s}}\right) \longrightarrow H F_{\check{C}, a_{-}+a_{s}, a_{+}}^{p}\left(H_{\check{s}}\right)
$$

is an isomorphism for each $\check{s} \geq v\left(a_{-}, a_{+}\right)$by Lemma 5.26 part (4) combined with Equation (5.18) (with $s=0$ ) and Lemma 3.8. Therefore we have a commutative diagram:

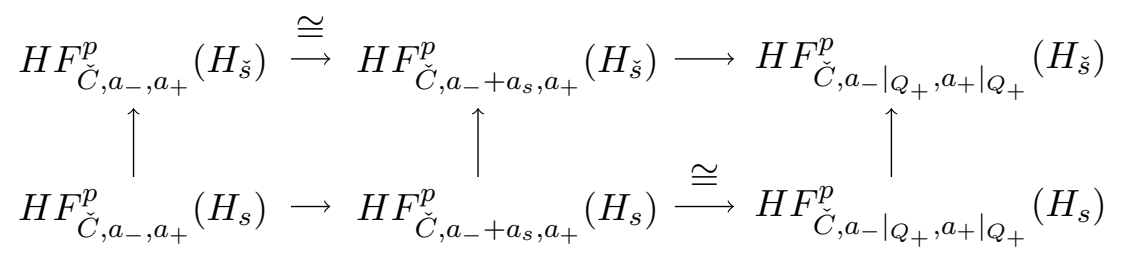

consisting of action maps (horizontal arrows) and continuation maps (vertical arrows) for each $\left(a_{-}, a_{+}\right) \in \operatorname{Sc}\left(Q_{-}\right) \times \operatorname{Sc}\left(Q_{+}\right)$satisfying (5.19) and each $s \geq 0$ and $\check{s} \geq s+v\left(a_{-}, a_{+}\right)$. Therefore we have a commutative diagram:

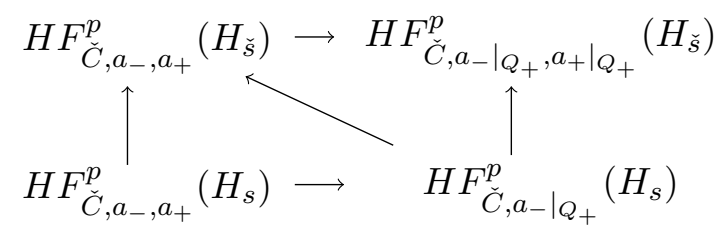

for each such $a_{-}, a_{+}, s, \check{s}$. This induces an isomorphism of directed systems

$$
\left(\underset{s}{\lim } H F_{\check{C}, a_{-}, a_{+}}^{p}\left(H_{s}\right)\right)_{\left(a_{-}, a_{+}\right) \in \operatorname{Sc}\left(Q_{-}\right) \times \operatorname{Sc}\left(Q_{+}\right)} \cong\left(\underset{s}{\lim } H F_{\check{C}, a_{-}^{\prime}, a_{+}^{\prime}}^{p}\left(H_{s}\right)\right)_{\left(a_{-}^{\prime}, a_{+}^{\prime}\right) \in \operatorname{Sc}\left(Q_{+}\right)^{2}} .
$$

Therefore by Proposition 5.5, the action map $\mathbb{S} H_{\breve{C}, Q_{-}, Q_{+}}^{*}(D \subset M) \longrightarrow \mathbb{S} H_{\breve{C}, Q_{+}, Q_{+}}^{*}(D \subset M)$ is an isomorphism in 2 -sys- $\Lambda_{\mathbb{K}}^{Q_{+},+}$. 
5.6. Transfer Isomorphisms between Index bounded Liouville Domains. In this subsection we give some sufficient conditions for a transfer map to be an isomorphism.

Definition 5.29. Let $\check{C}$ be a contact cylinder with associated Liouville domain $D$. A $L i$ ouville form associated to $\check{C}$ is a 1-form $\theta \in \Omega^{1}(D)$ satisfying $d \theta=\left.\omega\right|_{D}$ and $\left.\theta\right|_{D \cap C}=r_{C} \alpha_{C}$ where $r_{C}$ and $\alpha_{C}$ is the radial coordinate and contact form associated to $\check{C}$. A Liouville vector field associated to $\check{C}$ is the unique vector field $X_{\theta}$ on $D$ satisfying $i_{X_{\theta}} \omega=\theta$ for some Liouville form $\theta$ associated to $\check{C}$. The skeleton of a Liouville form $\theta$ associated to $\check{C}$ is the set of points $x \in D$ where the time $t$ flow of $x$ along $X_{\theta}$ exists for all time $t \in \mathbb{R}$. A skeleton of $\check{C}$ is the skeleton $S$ of $\theta$ for some Liouville for $\theta$ associated to $\check{C}$. We call $\theta$ a Liouville form associated to $S$.

Remark 5.30. Note that a skeleton $S$ of a contact cylinder is not unique. For instance one can obtain other skeletons of $\check{C}$ by pushing forward $S$ via a Hamiltonian diffeomorphism compactly supported in the interior of $D$. Also note that $D$ is a disjoint union of its skeleton and a tubular neighborhood $(-\infty, 0] \times \partial D$ given by flowing $\partial D$ backwards along $X_{\theta}$.

Definition 5.31. Let $\check{C}=[1-\epsilon, 1+\epsilon] \times C$ be a contact cylinder with associated Liouville domain $D$. Define $\omega_{\breve{C}}$ to be a $\check{C}$-compatible 2 -form with scaling constants 0 and 1 and which is equal to $\omega$ outside $D \cup([1,1+\epsilon / 2] \times C)$. We define $Q_{+}^{\check{C}} \subset H^{2}(M, D ; \mathbb{R}) \times \mathbb{R} \times \mathbb{R}$ to be the cone spanned by $\left(\left[\omega_{\check{C}}\right], 1,1\right)$ and $Q_{-}^{\check{C}}$ the cone spanned by $\left(\left[\omega_{\check{C}}\right], 1,1\right)$ and $\left(\left[\omega_{\check{C}}\right], 0,1\right)$.

Proposition 5.32. Let $\check{C}_{i}$ be a contact cylinder with associated Liouville domain $D_{i}$, Liouville form $\theta_{i}$ and skeleton $S_{i}$ of $\theta_{i}$ for $i=0,1,2,3,4$ so that

(1) $D_{i} \subset D_{j}$ and $\left.\theta_{j}\right|_{D_{i}}-\theta_{i}$ is exact for $i \geq j$,

(2) $\left.\theta_{0}\right|_{D_{2}}=\theta_{2}$ and $\left.\theta_{1}\right|_{D_{4}}=\theta_{4}$,

(3) $S_{i}$ is contained in the interior of $D_{4}$ for all $i$,

(4) and $D_{0}$ and $D_{3}$ are index bounded (See Figure 13).

Then the transfer map

$$
\mathbb{S} H_{\check{C}_{0}, Q_{-}, Q_{+}}^{*}\left(D_{0} \subset M\right) \longrightarrow \mathbb{S} H_{\check{C}_{0}, Q_{-}, Q_{+}}^{*}\left(D_{3} \subset M\right)
$$

is an isomorphism in 2 -sys- $\Lambda_{\mathbb{K}}^{Q_{+}}$where $Q_{ \pm}:=Q_{ \pm}^{\check{C}_{0}}$.

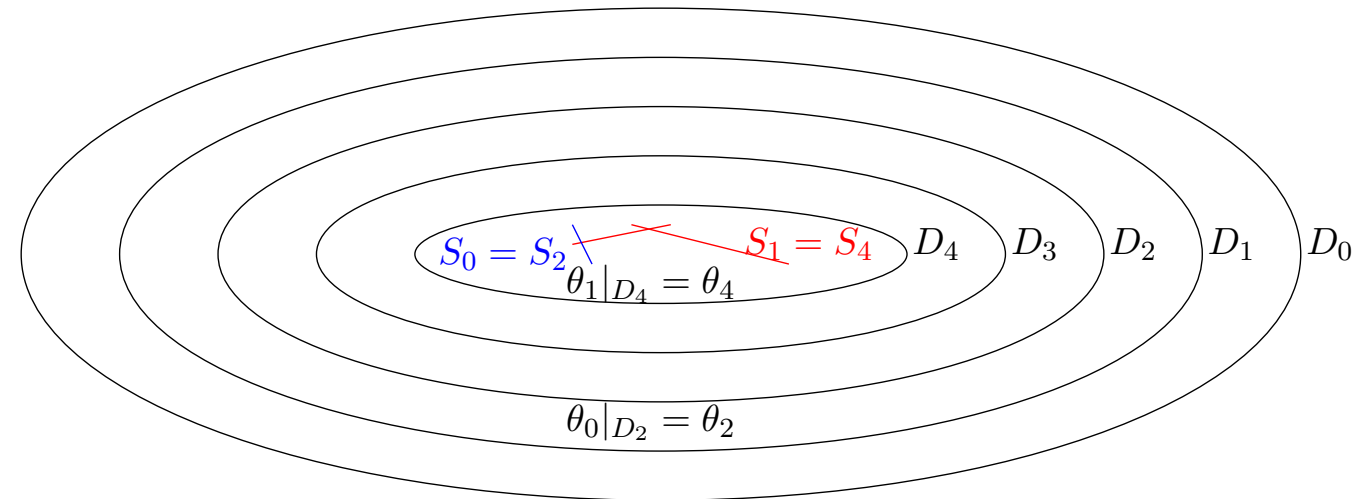

Figure 13. Schematic picture of the Liouville domains $D_{0}, \cdots, D_{4}$. 
Before we prove this proposition, we need a few preliminary lemmas.

Lemma 5.33. Let $\widehat{C}$ be a contact cylinder with associated Liouville domain $\widehat{D}$ and let $\check{C}:=$ $[1-\epsilon, 1+\epsilon] \times C$ be an index bounded contact cylinder with associated Liouville domain $D$ so that $D \cup \breve{C} \subset \widehat{D}$. Then the transfer map

$$
\mathbb{S} H_{\widehat{C}, Q_{-}^{\widehat{C}}, Q_{+}^{\widehat{C}}}^{*}\left(D \cup\left(\left[1, t^{\prime \prime}\right] \times C\right) \subset M\right) \longrightarrow \mathbb{S} H_{\widehat{C}, Q_{-}^{\widehat{C}}, Q_{+}^{\widehat{C}}}^{*}\left(D \cup\left(\left[1, t^{\prime}\right] \times C\right) \subset M\right)
$$

is an isomorphism for all $1 \leq t^{\prime} \leq t^{\prime \prime}<1+\epsilon$.

Proof. Define $D_{t}:=D \cup([1, t] \times C)$ for each $t \in[1,1+\epsilon)$. It is sufficient for us to show that for each $t \in[1,1+\epsilon)$, there exists a constant $0<\delta_{t}<1$ so that the transfer map

$$
S H_{\widehat{C}, Q_{-}, Q_{+}}^{*}\left(D_{t^{\prime \prime}} \subset M\right) \longrightarrow S H_{\widehat{C}, Q_{-}, Q_{+}}^{*}\left(D_{t^{\prime}} \subset M\right)
$$

is an isomorphism for each $t^{\prime}, t^{\prime \prime} \in[1,1+\epsilon)$ satisfying $t-\delta_{t} \leq t^{\prime} \leq t^{\prime \prime} \leq t+\delta_{t}$.

Fix $t \in[1,1+\epsilon)$. Let $\check{C}_{t} \subset \check{C}$ be a contact cylinder with associated Liouville domain $D_{t}$ and whose cylindrical coordinate is $r_{C} / t$ where $r_{C}$ is the cylindrical coordinate associated to $\check{C}$. Let $\left(H_{s}\right)_{s \in[0, \infty)}$ be as in Lemma 5.26 with $m=|p|+n+1$ for some $p \in \mathbb{Z}$ and with $\check{C}$ replaced by $\check{C}_{t}$. Let $A \subset \mathbb{R}$ be the set of action values $\mathcal{A}_{\widehat{C}, H_{0}}(\gamma)\left(\left[\omega_{\widehat{C}}\right], 1,1\right) \in \mathbb{R}$ and $\mathcal{A}_{\widehat{C}, H_{0}}(\gamma)\left(\left[\omega_{\widehat{C}}\right], 0,1\right) \in \mathbb{Z}$ where $\gamma$ runs over all capped 1-periodic orbits of $H_{0}$ whose associated 1-periodic orbit is contained in $D_{0}$. Since $A$ is closed and of measure 0 by Lemma 8.5, there exists $a \in \mathbb{R}$ and $\delta^{\prime}>0$ so that $\left(a+k-\delta^{\prime}, a+k+\delta^{\prime}\right) \cap A=\emptyset$ for all $k \in \mathbb{Z}$. Hence there exists a cofinal family $\left(a_{-}^{j}\right)_{j \in \mathbb{N}}$ in $\left(\operatorname{Sc}\left(Q_{-}^{\widehat{C}}\right), \geq\right)$, a cofinal family $\left(a_{+}^{j}\right)_{j \in \mathbb{N}}$ in $\left(\operatorname{Sc}\left(Q_{+}^{\widehat{C}}\right), \leq\right)$ and neighborhoods $N_{ \pm} \subset \operatorname{Sc}\left(Q_{ \pm}^{\widehat{C}}\right)$ of 0 so that for each $j \in \mathbb{N}$ and each capped 1-periodic orbit $\gamma$ of $H_{0}$ whose associated 1-periodic orbit is contained in $D_{0}$, we have that $a_{ \pm}^{j}(\gamma)-\left.\mathcal{A}_{\widehat{C}, H_{0}}(\gamma)\right|_{Q_{ \pm}^{\widehat{C}}} \notin$ $N_{ \pm}$. Therefore by Lemma 5.26 combined with Remarks 5.27 and 5.28 there is an increasing sequence of constants $\left(s_{j}\right)_{j \in \mathbb{N}}$ and a constant $\delta_{t} \in(0,1)$ so that

$$
\Gamma_{\widehat{C}, a_{-}^{j}, a_{+}^{j}}^{[-m, m]}\left(e^{\tau} H_{s}\right)=\Gamma_{\widehat{C}, a_{-}^{j}+n_{-}, a_{+}^{j}+n_{+}}^{[-m, m]}\left(e^{\tau} H_{s}\right),
$$

(See Definition 2.58) for all $j \in \mathbb{N}, s>s_{j}, n_{ \pm} \in N_{ \pm}$and $-\delta_{t} \leq \tau \leq \delta_{t}$ after possibly shrinking $N_{ \pm}$.

Let $\phi_{t}: M \longrightarrow M$ be the time $t$ flow of a vector field on $M$ equal to $r \frac{\partial}{\partial r}$ inside $\check{C}$ for each $t \in \mathbb{R}$. By Equation (5.23) and Lemma 3.6 combined with the fact that $\left.H_{s}\right|_{D}=\left.H_{0}\right|_{D}$ for each $s \in[0, \infty)$ we have that the natural continuation map

$$
H F_{\widehat{C}, a_{-}^{j}, a_{+}^{j}}^{p}\left(\left(\phi_{t^{\prime \prime}}\right)_{*} H_{s}\right) \longrightarrow H F_{\widehat{C}, a_{-}^{j}, a_{+}^{j}}^{p}\left(\left(\phi_{t^{\prime}}\right)_{*} H_{s}\right)
$$

is an isomorphism for each $t^{\prime}, t^{\prime \prime} \in[1,1+\epsilon)$ satisfying $-\delta_{t} \leq t^{\prime}<t^{\prime \prime} \leq \delta_{t}, j \in \mathbb{N}$ and $s>s_{j}$. This implies that the continuation map

$$
H F_{\widehat{C}, a_{-}^{j}, a_{+}^{j}}^{p}\left(H^{D_{t^{\prime \prime}}}\right) \longrightarrow H F_{\widehat{C}, a_{-}^{j}, a_{+}^{j}}^{p}\left(H^{D_{t^{\prime}}}\right)
$$

is an isomorphism for each $t^{\prime}, t^{\prime \prime} \in[1,1+\epsilon)$ satisfying $t-\delta_{t} \leq t^{\prime}<t^{\prime \prime} \leq t+\delta_{t}$ and $j \in \mathbb{N}$ where $H^{D_{\tau}} \in \mathcal{H}^{\mathbb{T}, \text {,.s.s. }}(\check{C}, \leq 0)$ (Definition 4.1) is equal to $\left(\phi_{\tau}\right)_{*}\left(H_{0}\right)$ inside $D_{\tau}$ and $\infty$ otherwise for each $\tau \in\left[-\delta_{t}, \delta_{t}\right]$. Hence by Lemma 2.40 and Proposition 5.5, the map (5.22) is an isomorphism.

We wish to reduce the proof of Proposition 5.32 to Lemma 5.33 above. In order to do this, we need to 'rearrange' our contact cylinders slightly. This is the purpose of the following two lemmas. 
Lemma 5.34. Let $\left(\check{C}_{i}\right)_{i=0,1}$ be contact cylinders with associated Liouville domains $\left(D_{i}\right)_{i=0,1}$ and skeletons $\left(S_{i}\right)_{i=0,1}$. Let $\left(\theta_{i}\right)_{i=0,1}$ be Liouville forms associated to $\left(S_{i}\right)_{i=0,1}$. Suppose that $D_{1} \subset D_{0}, S_{0}$ is contained in the interior of $D_{1}, S_{1} \subset S_{0}$ and that $\theta_{1}-\left.\theta_{0}\right|_{D_{1}}$ is exact. Suppose also that the time $t$ flow of $X_{\theta_{0}}$ sends $S_{1}$ to $S_{1}$ for all $t \in \mathbb{R}$. Then for each neighborhood $N$ of $S_{1}$, there is a Hamiltonian isotopy $\phi_{t}: M \longrightarrow M, t \in[0,1]$ compactly supported in the interior of $D_{1}$ satisfying $\phi_{0}=\mathrm{id}, \phi_{t}\left(S_{1}\right)=S_{1}$ for all $t \in[0,1]$ and $\phi_{1}\left(S_{0}\right) \subset N$.

Proof. Let $\phi_{t}^{i}: D_{i} \longrightarrow D_{i}$ be the time $t$ flow of the Liouville vector field $-X_{\theta_{i}}$ for each $i=0,1$. Since $S_{0}$ is contained in the interior of $D_{1}$, there exists $T>0$ so that $\phi_{t}^{0}\left(D_{0}\right) \subset D_{1}$ for all $t \geq T$. We can also assume $\phi_{t}^{1}\left(D_{1}\right) \subset N$ for all $t \geq T$. Define the embedding

$$
\iota_{t}: D_{0} \longrightarrow D_{1}, \quad \iota_{t}:=\phi_{t}^{1} \circ \phi_{2 T-t}^{0}
$$

for each $t \in[0, T]$. Then $\frac{d}{d t} \iota_{t}$ is a Hamiltonian vector field on $\iota_{t}\left(D_{0}\right)$ for all $t \in[0, T]$, $\iota_{0}\left(S_{0}\right)=S_{0}, \iota_{t}\left(S_{1}\right)=S_{1}$ for all $t \in[0, T]$ and $\iota_{T}\left(S_{0}\right) \subset N$. Extending this time dependent Hamiltonian vector field to a Hamiltonian vector field on $M$ compactly supported in $D_{1}$ completes our lemma.

Lemma 5.35. Let $\check{C}_{i}$ be a contact cylinder with associated Liouville domain $D_{i}$ and associated Liouville form $\theta_{i}$ for $i=0,1,2$. Suppose

- $D_{2} \subset D_{1} \subset D_{0}$,

- the skeleton of $D_{i}$ is contained in the interior of $D_{2}$ for each $i$,

- $\left.\theta_{0}\right|_{D_{2}}=\theta_{2}$ and $\left.\theta_{0}\right|_{D_{1}}-\theta_{1}$ is exact.

Then there is a 1 -form $\widehat{\theta}_{0}$ associated to $\check{C}_{0}$ so that $\widehat{\theta}_{0}-\theta_{0}$ is exact, $\left.\widehat{\theta}_{0}\right|_{D_{0}-D_{2}}=\left.\theta_{0}\right|_{D_{0}-D_{2}}$, the skeleton $\widehat{S}_{0}$ of $\widehat{\theta}_{0}$ contains the skeleton of $\theta_{1}$ and $\widehat{S}_{0}$ is contained in the interior of $D_{2}$. Also we can assume that the time $t$ flow along $X_{\widehat{\theta}_{0}}$ of $S_{1}$ is $S_{1}$ for all $t \in \mathbb{R}$.

Proof. Let $S_{i}$ be the skeleton of $\theta_{i}$ for each $i$. Then $S_{0}=S_{2}$. Choose a neighborhood $N$ of $S_{1}$ whose closure is contained in the interior of $D_{2}$. Let $f: D_{0} \longrightarrow \mathbb{R}$ be a smooth function with compact support in the interior of $D_{2}$ so that $\left.\theta_{0}\right|_{N}-\left.\theta_{1}\right|_{N}=\left.d f\right|_{N}$. Define $\widehat{\theta}_{0}:=\theta_{0}-d f$. This has the properties we want.

Proof of Proposition 5.32. Let $\left(D_{i}^{o}\right)_{i=0,1,2,3,4}$ be the interiors of $\left(D_{i}\right)_{i=0,1,2,3,4}$ respectively. By applying Lemma 5.35 twice we can find a Liouville form $\widehat{\theta}_{i}$ associated to $\check{C}_{i}$ with associated skeleton $\widehat{S}_{i}$ so that $\widehat{\theta}_{i}-\theta_{i}$ is exact for $i=0,1$ and so that $S_{3} \subset \widehat{S}_{1} \subset \widehat{S}_{0}$ and $\widehat{S}_{0} \subset D_{2}^{o}$ and $\widehat{S}_{1} \subset D_{4}^{o}$. Also we can assume that the time $t$ flow along $X_{\theta_{1}}$ of $S_{3}$ is contained in $S_{3}$ and the time $t$ flow along $X_{\widehat{\theta}_{0}}$ of $\widehat{S}_{1}$ is contained in $\widehat{S}_{1}$.

By Lemma 5.34, we can push forward $\widehat{\theta}_{0}$ by an appropriate Hamiltonian isotopy $\chi_{t}: M \longrightarrow$ $M, t \in[0,1]$ compactly supported in the interior of $D_{1}$ satisfying $\chi_{t}\left(\widehat{S}_{1}\right)=\widehat{S}_{1}$ for all $t \in[0,1]$ so that $\chi_{1}\left(\widehat{S}_{0}\right) \subset D_{4}^{o}$. Hence after replacing $\widehat{\theta}_{0}$ by its pushforward by $\chi_{1}$, we can still assume that $S_{3} \subset \widehat{S}_{1} \subset \widehat{S}_{0}$.

Let $\phi_{t}: D_{0} \longrightarrow D_{0}$ be the time $t$ flow of $-X_{\widehat{\theta}_{0}}$ and $\psi_{t}: D_{3} \longrightarrow D_{3}$ the time $t$ flow of $-X_{\theta_{3}}$. Since $S_{3} \subset \widehat{S}_{0} \subset D_{4}^{o}$, there are constants $T_{0}, T_{3}>0$ so that $D_{0}^{\prime}:=\phi_{T_{0}}\left(D_{0}\right) \subset D_{3}$ and $D_{3}^{\prime}:=\psi_{T_{3}}\left(D_{3}\right) \subset D_{0}^{\prime}$. Since $\cup_{t \in[0, T]} \phi_{t}\left(\partial D_{0}\right)$ is the image of a contact cylinder in $M$ for all $T>0$, we can apply Lemma 5.33 to show that the transfer map

$$
\mathbb{S} H_{\check{C}_{0}, Q_{-}^{\check{C}_{0}}, Q_{+}^{\check{C}_{0}}}^{*}\left(D_{0} \subset M\right) \longrightarrow \mathbb{S} H_{\check{C}_{0}, Q_{-}^{\check{C}_{0}}, Q_{+}^{\check{C}_{0}}}^{*}\left(D_{0}^{\prime} \subset M\right)
$$


is an isomorphism. Similarly

$$
\mathbb{S} H_{\check{C}_{0}, Q_{-}^{C_{0}}, Q_{+}^{\check{C}_{0}}}^{*}\left(D_{3} \subset M\right) \longrightarrow \mathbb{S} H_{\check{C}_{0}, Q_{-}^{C_{0}}, Q_{+}^{\check{C}_{0}}}^{*}\left(D_{3}^{\prime} \subset M\right)
$$

is an isomorphism. Since $D_{3}^{\prime} \subset D_{0}^{\prime} \subset D_{3} \subset D_{0}$ and since the composition of two transfer maps is a transfer map, this implies that the transfer map (5.20) is an isomorphism.

5.7. A Chain Complex For Symplectic Cohomology. In this section we construct a double system of chain complexes from Liouville domains associated index bounded contact cylinders. In the next section (see the proof of Theorem 5.39), we'll show that these double systems of chain complexes compute symplectic cohomology in some cases. Throughout this subsection we fix an index bounded (Definition 5.23) contact cylinder $\check{C}=[1-\epsilon, 1+\epsilon] \times C$ with associated Liouville domain $D$.

Lemma 5.36. Let $\left(Q_{-}^{m}, Q_{+}^{m}\right)_{m \in I}$ be a finite collection of $\check{C}$-interval domain pairs which are wide (Definition 2.58). For each $m \in I$ let $\left(a_{-}^{j, m}\right)_{j \in \mathbb{N}},\left(a_{+}^{j, m}\right)_{j \in \mathbb{N}}$ be a cofinal family of $\left(\operatorname{Sc}\left(Q_{-}^{m}\right), \geq\right)$ and $\left(\operatorname{Sc}\left(Q_{+}^{m}\right), \leq\right)$ respectively so that $a_{-}^{j+1, m} \leq a_{-}^{j, m}$ and $a_{+}^{j, m} \leq a_{+}^{j+1, m}$ for all $j \in \mathbb{N}$. Then there is an element $H_{D} \in \mathcal{H}^{\mathbb{T}, 1 . s .}(\check{C}, D, \leq 0)$ (Definition 4.1), a smooth family of autonomous Hamiltonians $\left(H_{s, t}\right)_{(s, t) \in[1, \infty) \times \mathbb{T}}$, a constant $s_{p} \geq 1$ and a subset $N_{p} \subset$ $D \cup([1,1+\epsilon / 8] \times C)$ which is open in $M$ for each $p \in \mathbb{N}$ so that

(1) $\left\{H_{s, \bullet}: s \geq s_{p}\right\}$ is a cofinal subset of $\mathcal{H}^{\mathrm{reg}}\left(<_{\check{C}} H_{D}, a_{-}^{p, m}, a_{+}^{p, m},[-p, p]\right)($ Definition 3.1) for each $m \in I$ where $H_{s, \bullet}:=\left(H_{s, t}\right)_{t \in \mathbb{T}}$ and $H_{s, \bullet}<_{\check{C}} H_{\check{s}, \bullet}$ for each $s<\check{s}$,

(2) all 1-periodic orbits of $H_{s, \bullet}$ of index in $[-p, p]$ have image contained in $N_{p}$ for each $s \geq s_{p}$ and

(3) $\left.H_{s, \bullet}\right|_{N_{p}}+1 / s=\left.H_{\breve{s}, \bullet}\right|_{N_{p}}+1 / \check{s}$ for each $s, \check{s} \geq s_{p}$ and each $p \in \mathbb{N}$.

Proof. Let $g:(-\infty, 1] \longrightarrow(-\infty,-1]$ be a continuous function so that

- $\left.g\right|_{(-\infty, 1)}$ is smooth and $g^{\prime}(x) \geq 0$ for $x<1$,

- $\left.g\right|_{(-\infty, 1-\epsilon / 16)}=-2$

- $g(x)=-1-\sqrt{1-x}$ for $x \geq 1-\epsilon / 32$.

Define

$$
\widetilde{g}:[1+\epsilon / 16, \infty) \longrightarrow(1, \infty), \quad \widetilde{g}(x):=1-g(2+\epsilon / 16-x) .
$$

Let $\left(a_{s}\right)_{s \in[1, \infty)}$ and $\left(b_{s}\right)_{s \in[1, \infty)}$ be smooth families of constants so that $\frac{d}{d s}\left(a_{s}\right)>0, \frac{d}{d s}\left(b_{s}\right)<$ $0, a_{s} \in(1-\epsilon / 32,1), b_{s} \in(1+\epsilon / 16,1+3 \epsilon / 32)$ for all $s \geq 1, a_{s} \rightarrow 1, b_{s} \rightarrow 1+\epsilon / 16$ and $g^{\prime}\left(a_{i}\right)$ is not equal to the length of any Reeb orbit of $\alpha_{C}$ for each $i \in \mathbb{N}_{>1}$. Such constants exist since the set of lengths of Reeb orbits has measure 0 in $\mathbb{R}$ by [Pop93, Proposition 3.2]. Let $f_{s}: \mathbb{R} \longrightarrow \mathbb{R}, s \in[1, \infty)$ be a smooth family of smooth functions satisfying:

- $f_{s}^{\prime} \geq 0, \frac{d}{d s} f_{s}(x)>0$,

- $f_{s}(x)=g(x)-\frac{1}{s}$ for $x \leq a_{s}, f_{s}(x)=\widetilde{g}(x)+s$ for $x \geq b_{s}$ and

- $\left.f_{s}\right|_{(1, \infty)}$ pointwise tends to infinity and the function $\left.f_{s}^{\prime}\right|_{\left[a_{s}, b_{s}\right]}$ uniformly tends to infinity as $s$ tends to infinity (See Figure 14).

Let $h:[1-\epsilon, 1+\epsilon] \longrightarrow \mathbb{R}$ satisfy

- $h^{\prime} \leq 0$,

- $\left.h\right|_{\left[1-\epsilon, 1+\frac{3 \epsilon}{32}\right]}=0$,

- $h(x)=-\frac{1}{4}\left(1+\frac{x}{1+\epsilon}\right)$ for all $x$ inside $[1+\epsilon / 8,1+\epsilon / 2]$,

- $\left.h\right|_{[1+3 \epsilon / 4,1+\epsilon]}=-1$ (See Figure 15). 


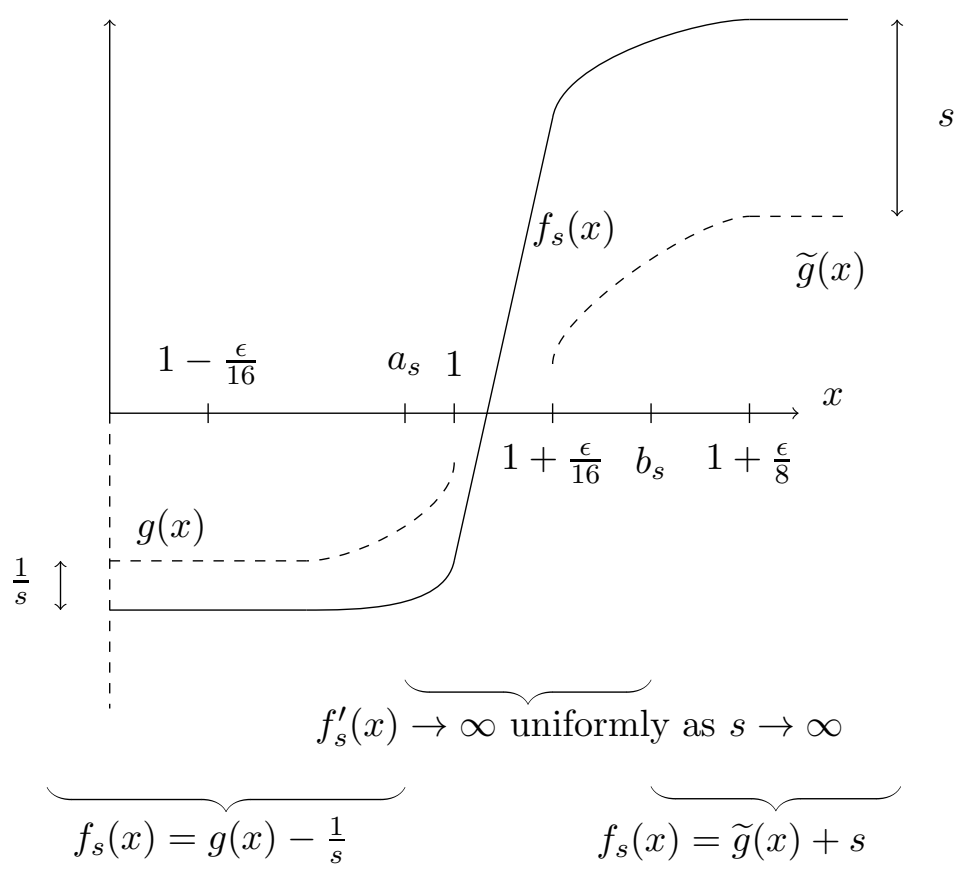

Figure 14. Graph of $f_{s}$ for each $s \in[1, \infty)$.

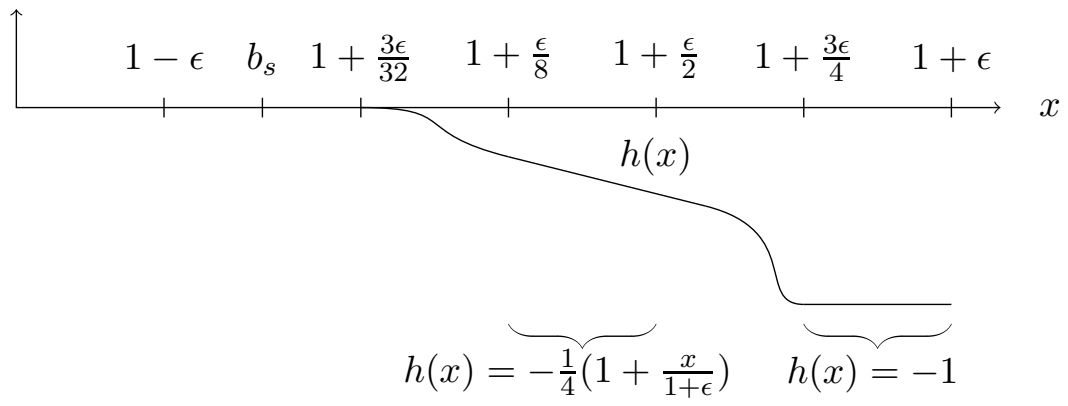

FiguRE 15. Graph of $h$.

Define

$$
K_{s}: M \longrightarrow \mathbb{R}, \quad K_{s}:=\left\{\begin{array}{ll}
-2-1 / s & \text { inside } D-\check{C} \\
f_{s}\left(r_{C}\right)+\frac{1}{s} h\left(r_{C}\right) & \text { inside } \check{C} \\
3+s-\frac{1}{s} & \text { otherwise }
\end{array}\right\}, \quad s \in[1, \infty) .
$$

Then $K_{s}<_{\check{C}} K_{\check{s}}$ for all $s<\check{s}$. Let $\rho: \mathbb{R} \longrightarrow[0,1]$ be a smooth function equal to 0 inside $(-\infty, 0]$ and 1 inside $[1, \infty)$. Define $B_{i}:=\left(a_{i}, a_{i+1}\right) \times C$ for each integer $i \geq 1$ and $B_{0}:=D-\left(\left[a_{1}, 1\right] \times C\right)$.

Since $\check{C}$ is index bounded, let $\Xi_{p}$ be larger than the length of the longest Reeb orbit of $\alpha_{C}$ of index in $[-p, p]$ for each $p \in \mathbb{N}$ so that $\left(\Xi_{p}\right)_{p \in \mathbb{N}}$ is increasing. Let $h_{p}:=$ 
$\max _{m \in I}$ height $\left(a_{-}^{p, m}, a_{+}^{p, m}\right)$ where height is given in Definition 2.58. For each $p \in \mathbb{N}$ choose $\check{s}_{p} \geq 1$ so that $\left.f_{s}^{\prime}\right|_{\left[a_{s}, b_{s}\right]}>\Xi_{p+n+1}$ for each $s \geq \check{s}_{p}$ and so that $\left(\check{s}_{p}\right)_{p \in \mathbb{N}}$ is increasing. Define

$$
s_{p}:=h_{p}+2+(1+\epsilon) \Xi_{p}+\check{s}_{p} .
$$

Then by Corollary 2.8 and Lemma 5.25 we have that for each $p \in \mathbb{Z}$ the 1 -periodic orbit associated to each element of $\Gamma_{\check{C}, a_{-}^{p, m}, a_{+}^{p, m}}^{[-p-n-1, p+n+1]}\left(H_{s}\right)$ (Definition 2.58) has image contained in $N_{p}:=D-\left(\left[a_{s_{p}}, 1\right] \times \check{C}\right)$ for each $m \in I$ and $s \geq s_{p}$. Also $H_{i}$ has no 1-periodic orbits in $\left\{r_{C}=\right.$ $\left.a_{i}\right\}$ for each $i \in \mathbb{N}$ since $g^{\prime}\left(a_{i}\right)$ is not the length of any Reeb orbit of $\alpha_{C}$ for each $i$. Therefore by repeatedly applying Lemma 8.2, we can find smooth Hamiltonians $W_{k}=\left(W_{k, t}\right)_{t \in \mathbb{T}}, k \in \mathbb{N}_{\geq 0}$ which are $C^{\infty}$ small (in particular, $C^{\infty}$ tending to 0 as $i$ tends to infinity) and non-negative and where $W_{i}$ has support inside $B_{i}$ for each $i$ so that $K_{i}+\sum_{k=0}^{i-1} W_{k} \in \cap_{j=1}^{q} \mathcal{H}^{\text {reg }}\left(<_{\check{C}}\right.$ $\left., a_{-}^{j, m}, a_{+}^{j, m},[-j, j]\right)$ for each $q, i \in \mathbb{N}$ satisfying $i \geq s_{q}$. Define $H_{s, \bullet}:=K_{s}+\sum_{k=0}^{\lfloor s\rfloor-1} \rho(s-k) W_{k}$ for each $s \geq 1$ where $\lfloor s\rfloor$ is the largest integer $\leq s$. Then $H_{s, \bullet}<_{\check{C}} H_{\check{s}, \bullet}$ for all $s<\check{s}$. Since $W_{k}$ is $C^{\infty}$ small for each $k$, we can assume that the associated 1-periodic orbit of every element of $\Gamma_{\check{C}, a_{-}^{p, m}, a_{+}^{p, m}}^{[-p, p]}\left(H_{s, \bullet}\right)$ has image in $N_{p}$ for each $m \in I$ (since the same is true for $H_{s}$ ). Similarly, we can assume that $\left.H_{s, \bullet}\right|_{D}<0$ for all $s \geq 1$ since $W_{k}$ is small for each $k \geq 0$. All such orbits are non-degenerate by construction and $\left.H_{s, \bullet}\right|_{N_{p}}+\frac{1}{s}=\left.H_{\check{s}, \bullet}\right|_{N_{p}}+\frac{1}{\check{s}}$ for each $s, \check{s} \geq s_{p}$. Hence property (1)-(3) holds, after possibly making $s_{p}$ larger so that $\frac{1}{s_{p}}$ is sufficiently small. This completes the lemma.

Definition 5.37. A double system of chain complexes is a double system (Definition 2.28) $W: I \times J \longrightarrow R$-mod of $\mathbb{Z}$-graded $R$ modules where each module $W(i, j)$ is a chain complex over $R$, where the differential has degree 1 and each morphism $W\left((i, j) \longrightarrow\left(i^{\prime}, j^{\prime}\right)\right)$ is a chain map. We define $\lim _{i} \lim _{\leftarrow} W(i, j)$ in the same way as Definitions 2.33 and 2.34, except that it is now a chain complex with a differential. Let $\left(Q_{-}^{m}, Q_{+}^{m}\right)_{m \in I},\left(a_{ \pm}^{j, m}\right)_{j \in \mathbb{N}}, H_{D},\left(H_{s, t}\right)_{(s, t) \in[1, \infty) \times \mathbb{T}}$, $\left(H_{s, \bullet}\right)_{s \in[1, \infty)},\left(s_{p}\right)_{p \in \mathbb{N}},\left(N_{p}\right)_{p \in \mathbb{N}}$ be as in Lemma 5.36. Choose $J \in \cap_{i \in \mathbb{N}} \operatorname{g}^{\operatorname{reg}}\left(H_{i, \bullet}, \check{C}\right)$. By Lemma 2.74 there exists

$$
H^{i,-+} \in \cap_{m \in I} \mathcal{H}^{\mathbb{R} \times \mathbb{T}}\left(\check{C}, a_{-}^{j, m}, a_{+}^{j^{\prime}, m}, H_{i, \bullet}, H_{i+1, \bullet}\right), \quad J^{i,-+} \in J^{\mathbb{R} \times \mathbb{T}, \operatorname{reg}}\left(H^{-+},(J, J), \check{C}\right)
$$

so that the chain level continuation map:

$$
\Phi_{i, m, j, j^{\prime}}^{q}:=\widetilde{\Phi}_{\check{H}^{i,-+, \breve{J}^{i,-+}}}^{q}: C F_{\check{C}, a_{-}^{j, m}, a_{+}^{j^{\prime}, m}}^{q}\left(H_{i, \bullet}\right) \longrightarrow C F_{\check{C}, a_{-}^{j, m}, a_{+}^{j^{j^{\prime}, m}}}^{q}\left(H_{i+1, \bullet}\right)
$$

is an isomorphism for each $i, q, j, j^{\prime} \in \mathbb{N}$ satisfying $j, j^{\prime} \leq q, i \geq s_{p},-p \leq q \leq p, p \in \mathbb{N}$ and $m \in I$. Finally define

$$
\begin{gathered}
\Phi_{i \rightarrow i^{\prime}, m, j, j^{\prime}}^{q}: C F_{\check{C}, a_{-}^{j, m}, a_{+}^{j^{\prime}, m}}^{q}\left(H_{i, \bullet}\right) \longrightarrow C F_{\check{C}, a_{-}^{j, m}, a_{+}^{j^{\prime}, m}}^{q}\left(H_{i^{\prime}, \bullet}\right), \\
\Phi_{i \rightarrow i^{\prime}, m, j, j^{\prime}}^{q}:=\Phi_{i^{\prime}-1, m, j, j^{\prime}}^{q} \circ \Phi_{i^{\prime}-2, m, j, j^{\prime}}^{q} \circ \cdots \circ \Phi_{i+1, m, j, j^{\prime}}^{q} \circ \Phi_{i, m, j, j^{\prime}}^{q}
\end{gathered}
$$

for all $i, q, j, j^{\prime}, i^{\prime} \in \mathbb{N}$ satisfying $j, j^{\prime} \leq q, i^{\prime} \geq i, i>s_{p},-p \leq q \leq p, p \in \mathbb{N}$ and $m \in I$ where such a map is the identity map for $i=i^{\prime}$. These maps give us a directed system $\left(C F_{\check{C}, a_{-}^{j, m}, a_{+}^{j^{\prime}, m}}^{q}\left(H_{i, \bullet}\right)\right)_{i \geq s_{p}}$ for each $q, j, j^{\prime} \in \mathbb{N}$ satisfying $j, j^{\prime} \leq q,-p \leq q \leq p, p \in \mathbb{N}$ and $m \in I$. Define

$$
W_{j, j^{\prime}, m}^{q}:=\underset{i \geq s_{p}}{\lim _{i}} C F_{\check{C}, a_{-}^{j, m}, a_{+}^{j^{\prime}, m}}^{q}\left(H_{i, \bullet}\right)
$$

for each $q, j, j^{\prime}, m$ as above. A compatible collection of double systems of chain complexes for $\mathbb{S} H_{\check{C}, Q_{-}^{m}, Q_{+}^{m}}^{*}(D \subset M), m \in I$ is defined to be the double systems of chain complexes 
$\left(W_{j, j^{\prime}, m}^{*}\right)_{j, j^{\prime} \in \mathbb{N}}, m \in I$ where the double system maps are chain level action maps and where $\mathbb{N}$ has the ordering $\geq$. If $I$ has just one element $m$ then such a double system is called a double system of chain complexes for $\mathbb{S} H_{\breve{C}, Q_{-}^{m}, Q_{+}^{m}}^{*}(D \subset M)$.

Remark 5.38. Suppose that $I=\{0,1\}$ and that $\left(Q_{-}^{1}, Q_{+}^{1}\right)$ is smaller than $\left(Q_{-}^{0}, Q_{+}^{0}\right)$ (Definition 2.75). Then $\mathbb{S} H_{\breve{C}, Q_{-}^{m}, Q_{+}^{m}}^{*}(D \subset M)$ is isomorphic to the double system $\left(H_{*}\left(W_{j, j^{\prime}, m}^{*}\right)\right)_{j, j^{\prime} \in \mathbb{N}}$ for each $m \in I$ by Lemma 2.40 and the action map

$$
\mathbb{S} H_{\check{C}, Q_{-}^{0}, Q_{+}^{0}}^{*}(D \subset M) \longrightarrow \mathbb{S} H_{\breve{C}, Q_{-}^{1}, Q_{+}^{1}}^{*}(D \subset M)
$$

is equal to the natural map

$$
\left(H_{*}\left(W_{j, j^{\prime}, 0}^{*}\right)\right)_{j, j^{\prime} \in \mathbb{N}} \longrightarrow\left(H_{*}\left(W_{j, j^{\prime}, 1}^{*}\right)\right)_{j, j^{\prime} \in \mathbb{N}}
$$

induced by the corresponding chain level action maps under this isomorphism of double systems.

\subsection{Changing Novikov Rings.}

Theorem 5.39. Let $\breve{C}$ be an index bounded contact cylinder with associated Liouville domain $D$ and let $\left(Q_{-}^{j}, Q_{+}^{j}\right)$ be a $\check{C}$-interval domain for $j=0,1$ so that $\left(Q_{-}^{1}, Q_{+}^{1}\right)$ is smaller than $\left(Q_{-}^{0}, Q_{+}^{0}\right)$ (Definition 2.75) and so that $\left(Q_{-}^{j}, Q_{+}^{j}\right)$ is wide for $j=0,1$ (Definition 2.58). Suppose that $\Lambda_{\mathbb{K}}^{Q^{1}}$ is a flat $\Lambda_{\mathbb{K}}^{Q^{0}}$-module and that $\underset{\lim }{\longleftrightarrow} \lim ^{1}\left(\mathbb{S} H_{\check{C}, Q_{-}^{1}, Q_{+}^{1}}^{*}(D \subset M)\right)=0$. Then the map

$$
S H_{C, Q_{-}^{0}, Q_{+}^{0}}^{*}(D \subset M) \otimes_{\Lambda_{\mathbb{K}}^{Q_{+}^{0}}} \Lambda_{\mathbb{K}}^{Q_{+}^{1}} \longrightarrow S H_{C, Q_{-}^{1}, Q_{+}^{1}}^{*}(D \subset M)
$$

induced by the corresponding action map is an isomorphism.

Proof of Theorem 5.39. Let $\left(W_{j, j^{\prime}, m}^{*}\right)_{j, j^{\prime} \in \mathbb{N}}, m=0,1$ be a compatible collection of double systems of chain complexes for $\mathbb{S} H_{\check{C}, Q_{-}^{m}, Q_{+}^{m}}^{*}(D \subset M), m=0,1$ as in Definition 5.37. By Remark 5.38 it is sufficient for us to show that the natural map

$$
\left(\underset{j}{\lim } \underset{j^{\prime}}{\lim } H_{*}\left(W_{j, j^{\prime}, 0}^{*}\right)\right) \otimes \Lambda_{\mathbb{K}}^{Q_{+}^{0}} \Lambda_{\mathbb{K}}^{Q_{+}^{1}} \longrightarrow \underset{j}{\lim } \underset{j^{\prime}}{\lim } H_{*}\left(W_{j, j^{\prime}, 1}^{*}\right)
$$

induced by chain level action maps is an isomorphism.

By Remark 5.38 combined with the fact that $\underline{\lim } \underset{\lim ^{1}}{\leftarrow} \mathbb{S} H_{\check{C}, Q_{-}^{1}, Q_{+}^{1}}^{p}(D \subset M)=0$, we get $\stackrel{\lim }{\lim ^{1}}\left(H_{*}\left(W_{j, j^{\prime}, 1}^{*}\right)\right)_{j, j^{\prime} \in \mathbb{N}}=0$. Hence by [Wei94, Theorem 3.5.8] combined with the fact that direct limits preserve short exact sequences and commute with homology and that $\Lambda_{\mathbb{K}}^{Q_{+}^{1}}$ is a flat $\Lambda_{\mathbb{K}}^{Q_{+}^{0}}$-module, we get a commutative diagram: 


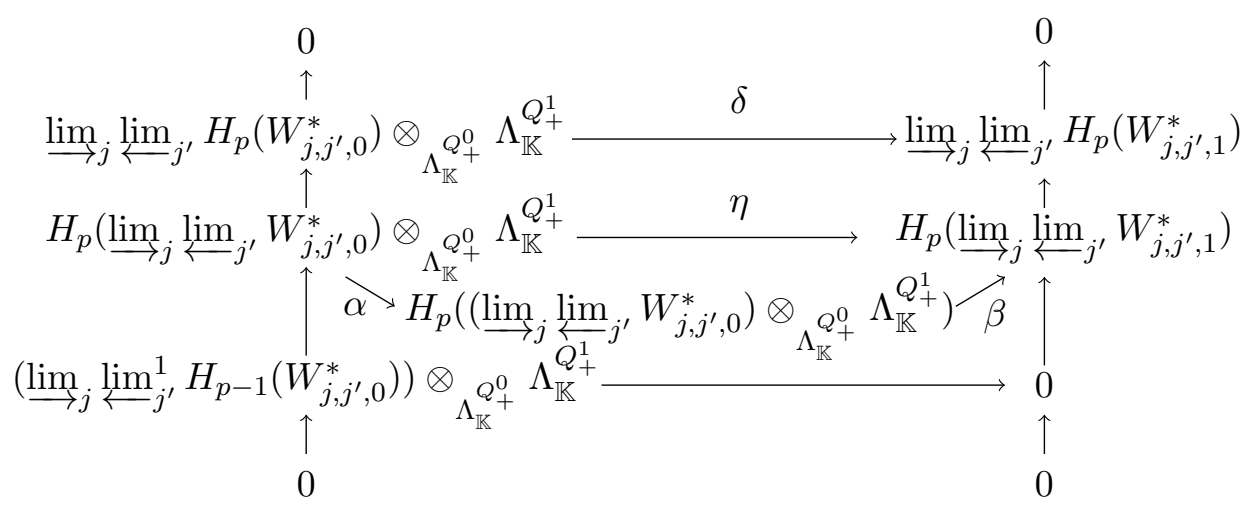

where the vertical morphisms form short exact sequences and the remaining maps are induced by chain level action maps for each $p \in \mathbb{Z}$. Since $\Lambda_{\mathbb{K}}^{Q_{+}^{1}}$ is a flat $\Lambda_{\mathbb{K}}^{Q_{+}^{0}}$-module, we have that $\alpha$ is an isomorphism. Also $\beta$ is an isomorphism since $\lim _{j} \lim _{j^{\prime}} W_{j, j^{\prime}, k}^{p}$ is a free finitely generated $\Lambda_{\mathbb{K}}^{Q_{+}^{k}}$-module for $k=0,1$ and $\beta$ sends the generators of one module to the other. This implies that the map (5.26) is an isomorphism.

\section{Symplectic Geometry of Projective Varieties and Singular Ample Divisors.}

6.1. Constructing Appropriate Kähler Forms. In order to show that birational Kähler manifolds have the same small quantum groups, we need to modify their Kähler forms so that they are identical on some large compact subset of a common open affine subset. This will enable us to show that various Hamiltonian Floer algebras are the same on both Calabi-Yau manifolds. This technical subsection is devoted to manipulating certain symplectic forms on smooth affine varieties in order to achieve this goal. It is also needed to ensure that this large compact subset contains a 'large' index bounded contact cylinder (see Section 6.2).

Definition 6.1. Let $\omega_{X}$ be a Kähler form on a complex manifold $\left(X, J_{X}\right)$. Then for any open subset $U \subset X$ and any smooth function $f: U \longrightarrow \mathbb{R}$, we define $\nabla_{\omega_{X}} f$ to be the unique vector field on $U$ satisfying $\omega_{X}\left(\nabla_{\omega_{X}} f, J_{X}(-)\right)=d f(-)$ (I.e. the gradient of $f$ with respect to the metric $\left.\omega_{X}\left(-, J_{X}(-)\right)\right)$.

Definition 6.2. Let $A$ be a smooth affine variety. Let $J_{A}: T A \longrightarrow T A$ be the complex structure on $A$. A smooth function $\rho: A \longrightarrow \mathbb{R}$ is exhausting if it is proper and bounded from below. Define $d^{c} \rho:=d \rho \circ J_{A}$. We say that $\rho$ is plurisubharmonic if $-d d^{c} \rho$ is a Kähler form. For each plurisubharmonic function $\rho$, we define $\omega_{\rho}:=-d d^{c} \rho$ and for each function $f: A \longrightarrow \mathbb{R}$, we define $\nabla_{\rho} f:=\nabla_{\omega_{\rho}} f$.

A smooth function $\rho: A \longrightarrow \mathbb{R}$ is an algebraic plurisubharmonic function if there exists a smooth projective variety $X$ compactifying $A$, a holomorphic line bundle $L$ over $X$ with a Hermitian metric $|\cdot|$ and a holomorphic section $s$ of $L$ so that $\rho=-\log (|s|), s^{-1}(0)=X-A$ and $\rho$ is plurisubharmonic.

A smooth function $\rho: A \longrightarrow \mathbb{R}$ is a partially algebraic plurisubharmonic function if there is an algebraic plurisubharmonic function $\rho_{\infty}: A \longrightarrow \mathbb{R}$ and a compact subset $K \subset A$ so that $\left.\rho\right|_{A-K}=\left.\rho_{\infty}\right|_{A-K}$ and if $\rho$ is plurisubharmonic.

Algebraic plurisubharmonic functions always exist since every affine variety can be compactified to a smooth projective variety $X$ by [Hir64]. One then can choose an ample line bundle $L$ together with a section $s$ satisfying $s^{-1}(0)=X-A$. Any ample line bundle admits 
a positive Hermitian metric $|\cdot|$ (e.g. a metric induced from the Fubini Study metric) which implies that $-\log (|s|)$ is plurisubharmonic. Having said that, in general we do not require that the metric $|\cdot|$ be positive outside $A$. Also note that all algebraic plurisubharmonic functions are exhausting.

The following technical lemma is needed to prove Corollaries 6.5 and 6.6 below. This lemma is basically about controlling the size of the derivatives of partially algebraic plurisubharmonic functions near infinity.

Lemma 6.3. Let $\rho_{0}, \rho_{1}$ be two partially algebraic plurisubharmonic functions on a smooth affine variety $A$. Define $\rho_{t}:=\rho_{0}+t \rho_{1}$ for each $t \in[0,1]$. Let $k$ be a positive integer. Then there is a compact subset $K \subset A$ so that

$$
d \rho_{t}\left(\nabla_{\rho_{t}} \rho_{t}\right)>\rho_{t}^{k} \quad \forall t \in[0,1]
$$

outside $K$. Also there is a vector field $V$ on $A$ so that

$$
d \rho_{0}(V)>0, \quad d \rho_{1}(V)>0
$$

outside $K$.

Proof. The key idea here is to reduce Equations (6.1) and (6.2) to a local estimate near each point at infinity. A similar thing has been done previously in the proof of [Sei08, Lemma 4.3].

First of all since we only require Equations (6.1) and (6.2) to hold outside a compact set, we can assume that $\rho_{j}$ is an algebraic plurisubharmonic function for all $j=0,1$. Therefore, by definition, for each $j \in\{0,1\}$ there is

- a smooth projective variety $X_{j}$ compactifying $A$,

- a holomorphic line bundle $\check{L}_{j}$ over $X_{j}$,

- a Hermitian metric $\|\cdot\|_{j}$ on $\check{L}_{j}$ and

- a holomorphic section $\check{s}_{j}$ of $\check{L}_{j}$

so that $\rho_{j}=-\log \left(\left\|\check{s}_{j}\right\|_{j}\right)$ and $\check{s}_{j}^{-1}(0)=X_{j}-A$. By the Hironaka resolution of singularities theorem [Hir64], there is a smooth projective variety $X$ compactifying $A$ and morphisms $\pi_{j}: X \longrightarrow X_{j}, j=0,1$ satisfying $\pi_{j}(a)=a$ for each $a \in A$ and each $j=0,1$. We can also assume that $X-A$ is a normal crossings variety (I.e. it is locally a transverse intersection of complex hypersurfaces). Let $L_{j}=\pi_{j}^{*} \check{L}_{j},|\cdot|_{j}=\pi_{j}^{*}\|\cdot\|_{j}$ and $s_{j}=\pi_{j}^{*} \check{s}_{j}$ be the corresponding pullbacks of our line bundle, metric and section to $X$ for each $j=0,1$. Then

$$
\rho_{j}=-\log \left(\left|s_{j}\right|_{j}\right) \text { and } s_{j}^{-1}(0)=X-A \quad \forall j=0,1 .
$$

In order to prove Equation (6.1) it is sufficient for us to show that for each $x \in X-A$, Equation (6.1) holds on a small neighborhood of $x$ since $X-A$ is compact. Similarly, in order to prove Equation (6.2), it is sufficient to show that there is a vector field $V_{x}$ defined in a neighborhood of $x$ satisfying $d \rho_{j}\left(V_{x}\right)>0, j=0,1$. This is because we can construct our desired vector field $V$ by patching together finitely many such vector fields $V_{x_{1}}, \cdots, V_{x_{k}}$ using partitions of unity.

Therefore fix $x \in X-A$. Choose a holomorphic coordinates $z_{1}, \cdots, z_{n}$ on a small chart $U_{x} \subset X$ centered at $x$ so that $(X-A) \cap U_{x}=\left\{\prod_{j=1}^{l} z_{j}=0\right\}$ for some $1 \leq l \leq n$. After shrinking $U_{x}$, we can choose trivializations of the line bundles $\left.L_{0}\right|_{U_{x}}$ and $\left.L_{1}\right|_{U_{x}}$. We will also assume that the coordinates $z_{1}, \cdots, z_{n}$ and trivializations above extend to a neighborhood of the closure of $U_{x}$ in order to ensure that $C^{1}$ bounds hold. For each $j=0,1$, there are smooth functions $\eta_{j}: U_{x} \longrightarrow \mathbb{R}$ so that $|\cdot|_{j}=e^{-\eta_{j}}|\cdot|$ with respect to the trivializations of $\left.L_{0}\right|_{U}$ and $\left.L_{1}\right|_{U}$ above. Also for each $j=0,1$, there are positive integers $a_{k}^{j}, k=1, \cdots, l$ and 
a holomorphic function $h_{j}: U_{x} \longrightarrow \mathbb{C}$ whose norm is bounded below by a positive constant so that $s_{j}=h_{j} \prod_{k=1}^{l} z_{k}^{a_{k}^{j}}$ with respect to the trivializations above. Hence

$$
\left.\left(\left|s_{j}\right|_{j}\right)\right|_{U_{x}}=e^{-\eta_{j}}\left|h_{j}\right| \prod_{k=1}^{l}\left|z_{k}\right|^{a_{k}^{j}} \quad \forall j=0,1 .
$$

Therefore

$$
\left.\rho_{j}\right|_{U_{x} \cap A}=\eta_{j}-\log \left(\left|h_{j}\right|\right)-\sum_{k=1}^{l} a_{k}^{j} \log \left(\left|z_{k}\right|\right) \quad \forall j=0,1 .
$$

Let $J_{X}$ be the complex structure on $X$. Let $g(-,-)$ be the standard Euclidean metric on $U_{x}$ and let $\|\cdot\|_{g}$ be the induced norm on the cotangent bundle $T^{*} U_{x}$. Let $\|\cdot\|_{\rho_{0}}$ be the induced norm on $T^{*} A$ coming from the metric $\omega_{\rho_{0}}\left(-, J_{X}(-)\right)$ on $A$. The metric $\omega_{\rho_{0}}\left(-, J_{X}(-)\right)$ smoothly extends to a $(0,2)$-tensor $g_{\rho_{0}}$ on $X$ (which may be degenerate along points of $X-A$ ). Since $g_{\rho_{0}}(Y, Y) \geq 0$ for all $Y \in T X$, there is a constant $c>0$ so that $g(Y, Y) \geq c g_{\rho_{0}}(Y, Y)$ for each $Y \in T U_{x}$. Hence $\left.c\|\cdot\|_{g}\right|_{A} \leq\|\cdot\|_{\rho_{0}}$. Therefore, by Equation (6.4),

$$
\begin{gathered}
\left.d \rho_{t}\left(\nabla_{\rho_{t}} \rho_{t}\right)\right|_{U_{x} \cap A}=\left\|d \rho_{t}\right\|_{\rho_{t}}^{2} \geq c^{2}\left\|d \rho_{t}\right\|_{g}^{2} \\
\geq \sum_{k=1}^{l} \frac{c^{2}}{\left|z_{k}\right|^{2}}\left\|\left(a_{k}^{0}+t a_{k}^{1}\right) d\left(\left|z_{k}\right|\right)\right\|_{g}^{2}- \\
\sum_{k=1}^{l} \frac{2 c^{2}}{\left|z_{k}\right|}\left(\left\|\left(a_{k}^{0}+t a_{k}^{1}\right) d\left(\left|z_{k}\right|\right)\right\|_{g} \| d\left(\eta_{0}-\log \left(\left|h_{0}\right|\right)+t d\left(\eta_{1}-\log \left(\left|h_{1}\right|\right)\right) \|_{g}^{2}\right), \quad \forall t \in[0,1] .\right.
\end{gathered}
$$

Hence Equation (6.1) holds near $x$ since the function $\frac{1}{y^{2}}$ grows much faster than $-\log (y)$ and $\frac{1}{y}$ as $y \rightarrow 0_{+}$.

Let $x_{k}$ be the real part of $z_{k}$ and $y_{k}$ the imaginary part for each $k=1, \cdots, n$. Then $x_{1}, y_{1}, \cdots, x_{n}, y_{n}$ are real coordinates on $U_{x}$. Now define the vector field $V_{x}:=-\sum_{k=1}^{n}\left(x_{k} \frac{\partial}{\partial x_{k}}+\right.$ $\left.y_{k} \frac{\partial}{\partial y_{k}}\right)$ on $U_{x}$. Then by Equation (6.4),

$$
d \rho_{j}\left(V_{x}\right)=d\left(\eta_{j}-\log \left(\left|h_{j}\right|\right)\right)\left(V_{x}\right)+\sum_{k=1}^{l} a_{k}^{j}, \quad \forall j=0,1
$$

which is positive in a neighborhood $\check{U}_{x}$ of $x$ since the $\|\cdot\|_{g}$ norm of $d\left(\eta_{j}-\log \left(\left|h_{j}\right|\right)\right)$ is bounded and the $g$-norm of $V_{x}$ tends to 0 as we approach $x$. Choose points $x^{1}, \cdots, x^{k}$ in $X-A$ so that $\check{U}_{x^{1}}, \cdots, \check{U}_{x^{k}}$ cover $X-A$. Choose a partition of unity $f: A \longrightarrow[0,1], f_{j}: \check{U}_{j} \longrightarrow[0,1]$, $j=1, \cdots, k$, subordinate to the cover $A, \check{U}_{x^{1}}, \cdots, \check{U}_{x^{k}}$ of $X$. Define $V:=\sum_{j=1}^{k} f_{j} V_{x_{j}}$. Then since Equation (6.5) holds inside $\check{U}_{x}$ for each $x \in X-A$, we get that $V$ satisfies Equation (6.2) outside a compact subset of $X$.

The following corollary of Lemma 6.3 will be used to construct appropriate Kähler forms on birational Calabi-Yau manifolds so that they coincide on some large compact set of some common affine variety. This will be used in the proof of Theorem 1.2 in Section 7 below. Before we state this corollary, we need a preliminary definition.

Definition 6.4. Let $\rho: A \longrightarrow \mathbb{R}$ be a plurisubharmonic function on an affine variety $A$. Let $\phi_{t}: A \longrightarrow A$ be the time $t$ flow of $-\nabla_{\rho} \rho$ for each $t \geq 0$. The skeleton of $\rho$ is the subset

$$
\cap_{t>0} \phi_{t}(A) \subset A \text {. }
$$


Note that the complement of the skeleton of $\rho$ is diffeomorphic to a product $\mathbb{R} \times Y$ where $\{r\} \times Y$ is a level set of $\rho$ for each $r \in \mathbb{R}$. As a result, one can think of $A$ as a 'cylindrical end' $\mathbb{R} \times Y$ with the skeleton 'glued' to one side (See Figure 16).

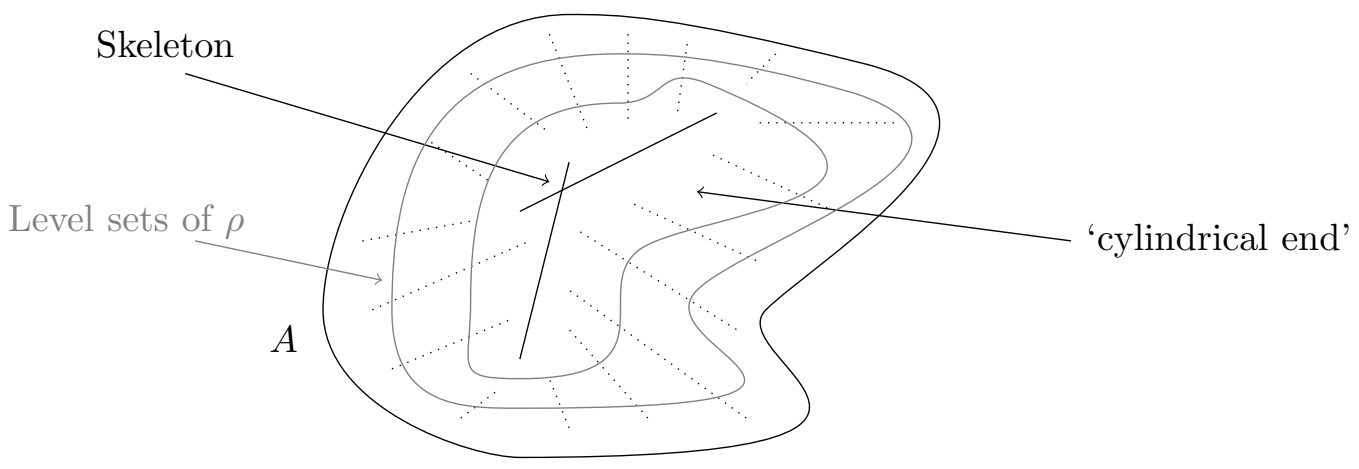

FiguRE 16. Skeleton of $A$.

Corollary 6.5. Let $\rho_{0}, \rho_{1}$ be two partially algebraic plurisubharmonic functions on a smooth affine variety $A$. Then for any compact subset $K \subset A$ there is a third partially algebraic plurisubharmonic function $\rho: A \longrightarrow \mathbb{R}$, a compact set $Q$ containing $K$ and constants $\kappa_{1}, \kappa_{2} \in$ $\mathbb{N}$ so that

- $\left.\rho\right|_{K}=\left.\rho_{1}\right|_{K}$,

- $\rho$ is equal to $\kappa_{1}\left(\rho_{0}-\log \kappa_{2}\right)$ outside $Q$ and

- the skeleton of $\rho$ is equal to the skeleton of $\rho_{1}$.

Proof. By Lemma 6.3 there is a vector field $V$ on $A$ and a compact subset $Q^{\prime} \subset A$ so that $d \rho_{0}(V)>0$ and $d \rho_{1}(V)>0$ outside $Q^{\prime}$. By enlarging $Q^{\prime}$, we can assume that it contains $K$. Let $\alpha:=\max \left(\left.\rho_{0}\right|_{Q^{\prime}}\right)+\log (2)$. Choose a compact subset $Q \subset A$ whose interior contains $Q^{\prime}$ and so that $\rho_{0}$ is greater than $\max (\alpha, 2)$ outside a compact subset of the interior of $Q$. Choose $\kappa_{2} \in \mathbb{N}$ so that $\max \left(\left.\rho_{0}\right|_{Q^{\prime}}\right)<\log \left(\kappa_{2}\right)<\max (\alpha, 2)$. Then

$$
\left.\rho_{0}\right|_{Q^{\prime}}-\log \left(\kappa_{2}\right)<0,\left.\quad \rho_{0}\right|_{\overline{A-Q}}-\log \left(\kappa_{2}\right)>0 .
$$

Hence we can choose an integer $\kappa_{1} \gg 1$ so that $f:=\kappa_{1}\left(\rho_{0}-\log \left(\kappa_{2}\right)\right)$ satisfies $\left.f\right|_{Q^{\prime}}<\left.\rho_{1}\right|_{Q^{\prime}}$ and $f>\rho_{1}$ outside a compact subset of the interior of $Q$. Then by [CE12, Propositions 3.20], we can smooth the function $\max \left(\rho_{1}, f\right)$ to a plurisubharmonic function $\rho: A \longrightarrow \mathbb{R}$ so that $\left.\rho\right|_{Q^{\prime}}=\left.\rho_{1}\right|_{Q^{\prime}},\left.\rho\right|_{A-Q}=\left.f\right|_{A-Q}$ and $d \rho(V)>0$ outside a compact subset of the interior of $Q^{\prime}$. Then $\rho$ has the required properties.

The following technical corollary of Lemma 6.3 will be used in the proof of Proposition 6.7 to construct certain index bounded contact cylinders.

Corollary 6.6. Let $\rho_{0}, \rho_{1}$ be two partially algebraic plurisubharmonic functions on a smooth affine variety $A$ and let $K \subset A$ be a compact subset. Then there are constants $0<\delta \ll T \ll 1$, a compact set $Q \subset A$ containing $K$ and an exhausting plurisubharmonic function $\rho$ on $A$ satisfying

(1) $\left.\rho\right|_{Q}=\left.\rho_{0}\right|_{Q}$,

(2) $\rho$ is equal to $\rho_{0}+\delta \rho_{1}$ outside a large compact set and 
(3) for all $x \in A$, the time $T$ flow of $x$ along $-\nabla_{\rho} \rho$ is contained in $Q$ and is disjoint from $K$ if, in addition, $x \notin Q$.

Proof. Define $\rho_{t}:=\rho_{0}+t \rho_{1}$ for each $t \in[0,1]$. Let $\phi_{t, \tau}: A \longrightarrow A$ be the time $\tau$ flow of $-\nabla_{\rho_{t}} \rho_{t}$ for all $\tau \geq 0$ and $t \in[0,1]$. For each $\tau \geq 0$, define

$$
\widehat{A}_{\tau}:=\cup_{t \in[0,1]} \phi_{t, \tau}(A), \quad \check{A}_{\tau}:=\cap_{t \in[0,1]} \phi_{t, \tau}(A) .
$$

By Lemma 6.3,

$$
d \rho_{t}\left(\nabla_{\rho_{t}} \rho_{t}\right)>\rho_{t}^{2}, \quad \forall t \in[0,1]
$$

outside a compact subset of $A$. This implies that $\widehat{A}_{\tau}$ and $\check{A}_{\tau}$ are relatively compact subsets for each $\tau>0$ since $\left(\rho_{t}\right)_{t \in[0,1]}$ are exhausting functions. We also have the following properties:

(a) $\overline{\widehat{A}}_{\tau_{0}} \subset \widehat{A}_{\tau_{1}}$ and $\overline{\check{A}_{\tau_{0}}} \subset \check{A}_{\tau_{1}}$ for each $0 \leq \tau_{1}<\tau_{2}$.

(b) $\phi_{t, \tau_{0}}\left(\check{A}_{\tau_{1}}\right) \subset \widehat{A}_{\tau_{0}+\tau_{1}}$ and $\phi_{t, \tau_{0}}\left(\widehat{A}_{\tau_{1}}\right) \supset \check{A}_{\tau_{0}+\tau_{1}}$ for each $\tau_{0}, \tau_{1} \geq 0$.

Choose constants $1 \gg \tau_{0} \gg \tau_{1} \gg \tau_{2}>0$ so that $K \subset \check{A}_{4 \tau_{0}}, \overline{\widehat{A}_{\tau_{0}}} \subset \check{A}_{2 \tau_{1}}$ and $\overline{\widehat{A}_{\tau_{1}}} \subset \check{A}_{2 \tau_{2}}$. Define $T:=2 \tau_{0}$ and $Q:=\overline{\widehat{A}_{\tau_{0}}}$. Let $\beta: A \longrightarrow \mathbb{R}$ be a smooth function equal to 0 along $Q$ and 1 outside $\check{A}_{2 \tau_{1}}$. Define $\check{\rho}_{t}:=(1-\beta) \rho_{0}+\beta \rho_{t}$ for all $t \in[0,1]$. Since being plurisubharmonic is a $C^{2}$ open condition, there is a constant $\eta>0$ so that $\check{\rho}_{t}$ is plurisubharmonic for all $t \in[0, \eta]$. Let $\psi_{t, \tau}$ be the time $\tau$ flow of $-\nabla_{\check{\rho}_{t}} \check{\rho}_{t}$ for each $t \in[0, \eta]$. Since $\rho_{t}$ converges to $\rho_{0}$ in the $C_{\text {loc }}^{\infty}$ topology as $t \rightarrow 0$ and by (a), (b) above combined with the fact that $\widehat{A}_{\tau}, \check{A}_{\tau}$ are relatively compact for all $\tau>0$, there exists $\delta \in(0, \eta]$ small enough so that

$$
\psi_{\delta, \tau}\left(\check{A}_{\tau_{2}}\right) \subset Q, \quad \psi_{\delta, \tau}\left(\check{A}_{\tau_{2}}-Q\right) \cap K=\emptyset, \quad \forall \tau \in\left\{T-\tau_{1}, T\right\} .
$$

Since $\beta^{-1}([0,1)) \subset \check{A}_{2 \tau_{1}}$ and by (a), (b), we have

$$
\psi_{\delta, \tau_{1}}\left(A-\widehat{A}_{\tau_{1}}\right)=\phi_{\delta, \tau_{1}}\left(A-\widehat{A}_{\tau_{1}}\right) \subset \widehat{A}_{\tau_{1}}-\check{A}_{2 \tau_{1}}
$$

and hence by Equation (6.7),

$$
\psi_{\delta, T}(A) \subset Q, \quad \psi_{\delta, T}(A-Q) \cap K=\emptyset .
$$

Hence $\rho:=\check{\rho}_{\delta}$ satisfies the properties we want.

6.2. Constructing Index Bounded Contact Cylinders. The main aim of this section is to prove the proposition below which constructs appropriate index bounded contact cylinders in Calabi-Yau manifolds. Recall that a contact cylinder is a codimension 0 symplectic embedding of a subset $\check{C}=[1-\epsilon, 1+\epsilon] \times C$ of a symplectization of a contact manifold $C$ which bounds a Liouville domain $D$ (see Definition 2.3 for more precise details). Such a contact cylinder is index bounded if for each $m>0$, there is a constant $\mu_{m}>0$ so that each Reeb orbit in $C$ of index in $[-m, m]$ has length $<\mu_{m}$ (see Definition 5.23).

Proposition 6.7. Let $X$ be a smooth projective variety satisfying $c_{1}(X)=0$ and let $A \subset X$ be an affine open subset. Let $\rho: A \longrightarrow \mathbb{R}$ be a partially algebraic plurisubharmonic function as in Definition 6.2 so that $-d d^{c} \rho$ extends to a Kähler form $\omega_{X}$ on $X$. Then for any compact subset $K \subset A$, there exists an index bounded contact cylinder $\check{C}$ of $\left(X, \omega_{X}\right)$ inside $A-K$ whose associated Liouville domain $D$ satisfies $K \subset D \subset A$. Also $\check{C}$ contains a contact cylinder $\check{C}_{0} \subset \check{C}$ whose associated Liouville domain domain $D_{0}$ contains $D$ and where $-\left.d^{c} \rho\right|_{D_{0}}$ is a Liouville form associated to $\check{C}_{0}$. 
The idea of the proof is to blow up $X$ so that the complement of $A$ is a smooth normal crossing divisor. One then finds a nice symplectic neighborhood of this divisor (after deformation) and constructs an index bounded contact cylinder in this neighborhood. As a result we need some technical definitions and lemmas about the symplectic geometry of normal crossing divisors.

Before that, we need a lemma about Conley-Zehnder indices of matrices.

Lemma 6.8. Let $\mathbb{R}^{2 n}$ be the standard symplectic vector space and let $L \subset \mathbb{R}^{2 n}$ be a fixed linear Lagrangian subspace. Let $A:=\left(A_{t}\right)_{t \in[0,1]}$ be a smooth family of symplectic matrices on $\mathbb{R}^{2 n}$ so that $A_{t}(x)=x$ for all $x \in L$. Then $C Z(A) \in[-2 n, 2 n]$.

Proof. Let $x_{1}, x_{2}, \cdots, x_{n}, y_{1}, \cdots, y_{n}$ be a basis of $\mathbb{R}^{2 n}$ so that the standard linear symplectic form on it is $\Omega_{\text {std }}=\sum_{i=1}^{n} x_{i}^{*} \wedge y_{i}^{*}$ where $x_{1}^{*}, \cdots, x_{n}^{*}, y_{1}^{*}, \cdots, y_{n}^{*}$ is the corresponding dual basis. We can also assume that $L=\left\{y_{1}^{*}=y_{2}^{*}=\cdots=y_{n}^{*}=0\right\}$. Since $A_{t}\left(x_{i}\right)=x_{i}$ for each $i=1, \cdots, n$, there exists a smooth family of $n \times n$ symmetric matrices $\left(B_{t}\right)_{t \in[0,1]}$ so that

$$
A_{t}=\left(\begin{array}{ll}
\text { id } & -B_{t} \\
0 & \text { id }
\end{array}\right), \quad \forall t \in[0,1]
$$

with respect to the basis above where id is the $n \times n$ identity matrix. Since the space of symmetric $n \times n$ matrices is convex, we have that the above path of matrices is homotopic relative to its endpoints to the catenation of the paths $A^{0}=\left(A_{t}^{0}\right)_{t \in[0,1]}$ and $A^{1}=\left(A_{t}^{1}\right)_{t \in[0,1]}$ where:

$$
A_{t}^{0}:=\left(\begin{array}{ll}
\mathrm{id} & -(1-t) B_{0} \\
0 & \mathrm{id}
\end{array}\right), \quad A_{t}^{1}:=\left(\begin{array}{ll}
\mathrm{id} & -t B_{1} \\
0 & \mathrm{id}
\end{array}\right), \quad t \in[0,1] .
$$

Therefore by (CZ3) and (CZ4),

$$
C Z(A)=C Z\left(A^{0}\right)+C Z\left(A^{1}\right) .
$$

Also by (CZ3), $C Z\left(A^{0}\right)=-C Z\left(\left(A_{1-t}^{0}\right)_{t \in[0,1]}\right)$, and hence by (CZ5) and Equation (6.8),

$$
C Z\left(A^{0}\right)=-\frac{1}{2} \operatorname{Sign}\left(B_{0}\right), \quad C Z\left(A^{1}\right)=\frac{1}{2} \operatorname{Sign}\left(B_{1}\right) .
$$

Combining this with Equation (6.9) gives us

$$
C Z\left(\left(A_{t}\right)_{t \in[0,1]}\right)=\frac{1}{2}\left(\operatorname{Sign}\left(B_{1}\right)-\operatorname{Sign}\left(B_{0}\right)\right) \in[-2 n, 2 n] .
$$

Definition 6.9. [FTMZ14, Definition 2.1]. Let $\left(\Delta_{i}\right)_{i \in S}$ be a finite collection of transversally intersecting closed codimension 2 symplectic submanifolds of a compact symplectic manifold $\left(W, \omega_{W}\right)$ so that $\Delta_{I}:=\cap_{i \in I} \Delta_{i}$ is a symplectic submanifold for all $I \subset S$ (our convention is that if $I=\emptyset$ then $\left.\Delta_{I}=W\right)$. The symplectic orientation on $\Delta_{I}$ is the orientation on $\Delta_{I}$ induced by the symplectic form. Let $N \Delta_{I}$ be the normal bundle of $\Delta_{I}$ for each $I \subset S$. The intersection orientation on $\Delta_{I}$ is the orientation on $\Delta_{I}$ coming from the symplectic orientation on its normal bundle induced by the splitting $N \Delta_{I}=\left.\oplus_{i \in I} N \Delta_{i}\right|_{\Delta_{I}}$ and the symplectic orientation on $M$.

A symplectic crossings divisor or $S C$ divisor inside a symplectic manifold $\left(W, \omega_{W}\right)$ is a finite collection $\left(\Delta_{i}\right)_{i \in S}$ of transversally intersecting closed submanifolds of $W$ as above so that $\Delta_{I}$ is symplectic and so that the symplectic orientation and the intersection orientation of $\Delta_{I}$ agree for all $I \subset S$. 
One of the main examples of an SC divisor to keep in mind is a union of transversally intersecting complex hypersurfaces in a Kähler manifold. The definition above should be thought of a symplectic version of such a union of complex hypersurfaces. This definition is more flexible and will enable us to control what the symplectic structure looks like near $\cup_{i \in S} \Delta_{i}$ after deforming $\left(\Delta_{i}\right)_{i \in S}$. For instance, it would be nice for these $\Delta_{i}$ 's to be symplectically orthogonal to each other after deformation. The condition ensuring that the symplectic and intersection orientation agree is crucial for such a deformation to exist. An example where this does not happen is if $W=T^{*} \mathbb{R}^{2}$ and if $\Delta_{1}$ is the graph of $x d y$ and $\Delta_{2}$ the graph of $y d x$ where $x, y$ are the standard coordinates on $\mathbb{R}^{2}$. These are two symplectic hypersurfaces which intersect negatively at the origin. There is no way of isotoping these linear symplectic subspaces so that they intersect orthogonally with respect to the symplectic form. A more sophisticated example where this orientation condition fails is contained in [FTMZ14, Example 2.7 .

Definition 6.10. Let $Q \subset W$ be a submanifold of a manifold $W$. A tubular neighborhood of $Q$ is a smooth fibration $\pi_{Q}: U_{Q} \longrightarrow Q$ so that

(1) $U_{Q} \subset W$ is an open subset containing $Q$,

(2) there is a metric $g$ on $W$ so that $\pi_{Q}=\pi_{D Q} \circ \exp ^{-1}$ where

$$
D Q:=\left\{\left.v \in T W\right|_{x}: x \in Q, g(v, v)<1, g(v, w)=\left.0 \forall w \in T Q\right|_{x}\right\}
$$

is the unit disk normal bundle, $\pi_{D Q}: D Q \longrightarrow Q$ is the natural projection map and $\exp : D Q \longrightarrow W$ is the exponential map with respect to $g$ so that

(3) exp is an embedding and $\exp (D Q)=U_{Q}$.

Recall that an Ehresmann connection on a smooth fiber bundle $\pi: E \longrightarrow B$ is a subbundle $H \subset T E$ so that $\left.D \pi\right|_{\left.H\right|_{x}}:\left.\left.H\right|_{x} \longrightarrow T B\right|_{\pi(x)}$ is an isomorphism for each $x \in E$. Such a connection is complete if for every smooth embedding $p:[0,1] \longrightarrow B$ and every $x \in \pi^{-1}(p(0))$, there is a unique lift $\widetilde{p}:[0,1] \longrightarrow E$ of $p$ tangent to $H$. In other words, points in $E$ do not parallel transport to infinity in finite time.

If $\pi: E \longrightarrow B$ is a smooth fibration and $\Omega$ a closed 2-form on $E$ making the fibers of $E$ symplectic then the associated symplectic connection is defined to be the Ehresmann connection consisting of vectors $\Omega$-orthogonal to the fibers. I.e.

$$
H_{\Omega}:=\left\{\left.Q \in T E\right|_{x}: x \in E, \Omega(Q, A)=\left.0 \forall A \in \operatorname{ker}(D \pi)\right|_{x}\right\} .
$$

Let $I$ be a finite set. A symplectic $U(1)^{I}$ neighborhood of a symplectic submanifold $Q \subset W$ of a symplectic manifold $\left(W, \omega_{W}\right)$ is a tubular neighborhood $\pi_{Q}: U_{Q} \longrightarrow Q$ of $Q$ where

(1) the fibers are symplectic submanifolds symplectomorphic to $\prod_{i \in I} \mathbb{D}_{i}(\epsilon)$ where $\mathbb{D}_{i}(\epsilon) \subset$ $\mathbb{C}$ is the open symplectic disk of radius $\epsilon$ labeled by $i \in I$,

(2) the fiber bundle $\pi_{Q}$ has structure group $U(1)^{I}:=\prod_{i \in I} U(1)$ given by rotating such disks in the natural way and

(3) the associated symplectic connection is complete and the parallel transport maps induced by the symplectic connection respect the above structure group (in other words, parallel transport maps between fibers in a $U(1)^{I}$ trivialization are elements of $\left.U(1)^{I}\right)$.

For $I^{\prime} \subset I$, let $U_{Q}^{I^{\prime}} \subset U_{Q}$ be the subset of points fixed under the $U(1)^{I^{\prime}} \subset U(1)^{I}$ action. For each $I^{\prime} \subset I$, the $U(1)^{I^{\prime}}$-bundle associated to $\pi_{Q}$ is the fibration $\pi_{Q}^{I^{\prime}}: U_{Q} \longrightarrow U_{Q}^{I^{\prime}}$ whose 
restriction to each fiber in a $U(1)^{I}$ trivialization is the natural projection map

$$
\prod_{i \in I} \mathbb{D}_{i}(\epsilon) \longrightarrow \prod_{i \in I^{\prime}} \mathbb{D}_{i}(\epsilon)
$$

and with induced structure group $U(1)^{I^{\prime}}$. This is naturally a symplectic $U(1)^{I^{\prime}}$ neighborhood of $U_{Q}^{I^{\prime}}$ inside $U_{Q}$.

A standard tubular neighborhood of an SC divisor $\left(\Delta_{i}\right)_{i \in S}$ consists of a symplectic $U(1)^{I}$ neighborhood $\pi_{I}: U_{I} \longrightarrow \Delta_{I}$ for each $I \subset S$ so that

(1) $U_{I} \cap U_{I^{\prime}}=U_{I \cup I^{\prime}}$ for all $I, I^{\prime} \subset S$,

(2) $\pi_{I^{\prime}}\left(U_{I}\right)=U_{I} \cap \Delta_{I^{\prime}}$ for all $I^{\prime} \subset I \subset S$ and

(3) the $U(1)^{I^{\prime}}$-bundle associated to $\pi_{I}$ is equal to $\left.\pi_{I^{\prime}}\right|_{U_{I}}: U_{I} \longrightarrow U_{I} \cap \Delta_{I^{\prime}}$ as fiber bundles with structure groups $U_{Q}^{I^{\prime}}$ for all $I^{\prime} \subset I \subset S$.

The radius of this standard tubular neighborhood is $\epsilon$. The radial coordinate $r_{i}: U_{i} \longrightarrow \mathbb{R}$ corresponding to $\Delta_{i}$ is the map whose restriction to each fiber $\mathbb{D}_{i}(\epsilon)$ of a $U(1)^{\{i\}}$-trivialization of $\pi_{i}$ is the standard radial coordinate on this disk.

Definition 6.11. Let $\left(W, \omega_{W}\right)$ be a closed symplectic manifold of dimension $2 n$ and let $\left(\Delta_{i}\right)_{i \in S}$ be a symplectic SC divisor in $W$. Define $W^{o}:=W-\cup_{j \in S} \Delta_{j}$. Let $\theta \in \Omega^{1}\left(W^{o}\right)$ satisfy $d \theta=\left.\omega_{W}\right|_{W^{o}}$. Let $N$ be a neighborhood of $\cup_{j \in S} \Delta_{j}$ which deformation retracts on to $\cup_{j \in S} \Delta_{j}$ and let $\beta: N \longrightarrow[0,1]$ be a compactly supported smooth function equal to 1 near $\cup_{j \in S} \Delta_{j}$. Let $\omega_{c}$ be the compactly supported closed 2 -form on $N$ equal to $\omega$ near $\cup_{j \in S} \Delta_{j}$ and $d(\beta \theta)$ inside $N \cap W^{o}$. Then since $N$ deformation retracts onto $\cup_{j \in S} \Delta_{j}$, the Lefschetz dual of $\omega_{c}$ is equal to $-\sum_{j} w_{j}\left[\Delta_{j}\right] \in H_{2 n-2}(N ; \mathbb{R})$ for unique real numbers $\left(w_{j}\right)_{j \in S}$. The wrapping number of $\theta$ around $\Delta_{j}$ is defined to be $w_{j}$ for each $j \in S$. We call $\left(\Delta_{j}\right)_{j \in S}$ a negatively wrapped divisor if there exists a 1-form $\theta$ on $W^{o}$ as above so that the wrapping number of $\theta$ around $W_{j}$ is negative for each $j \in S$.

The following lemma gives us an important example of a negatively wrapped divisor.

Lemma 6.12. Let $X$ be a complex projective variety and let $A \subset X$ be a codimension 0 affine subvariety so that $X-A$ is a union of transversally intersecting complex hypersurfaces $D_{1}, \cdots, D_{l}$. Let $\rho: A \longrightarrow \mathbb{R}$ be an exhausting plurisubharmonic function on $A$ as in Definition 6.2 so that $-d d^{c} \rho$ extends to a Kähler form $\omega_{X}$ on $X$. Then $\left(D_{i}\right)_{i=1}^{l}$ is a negatively wrapped divisor on $\left(X, \omega_{X}\right)$.

Proof. Let $\theta=-d^{c} \rho$. We will show that the wrapping number of $\theta$ around $D_{i}$ is negative for each $i \in\{1, \cdots, l\}$. Fix such a $D_{i}$. We will use a characterization of wrapping number in terms of embedded disks (See [McL16, Lemma 5.5]). Let $\mathbb{D} \subset \mathbb{C}$ be the closed unit disk and let $\iota: \mathbb{D} \hookrightarrow X$ be a holomorphic embedding so that

- $\iota^{-1}\left(D_{i}\right)=\{0\}$,

- $\iota(\mathbb{D})$ intersects $D_{i}$ transversally and

- $\iota^{-1}\left(D_{j}\right)=\emptyset$ for each $j \neq i$.

Since $\omega_{X}$ is a Kähler form and since $\mathbb{D}$ is contractible, there exists a smooth function $f$ : $\mathbb{D} \longrightarrow \mathbb{R}$ so that $\iota^{*} \omega_{X}=-d d^{c} f$. Since $-d d^{c}\left(\iota^{*} \rho-f\right)=0$, we get that $-d^{c}\left(\iota^{*} \rho-f\right)$ represents a De Rham cohomology class in $H^{1}(\mathbb{D}-\{0\} ; \mathbb{R})$. By [McL16, Lemma 5.5], this cohomology class determines the wrapping number $w_{i}$ of $\theta$ around $D_{i}$. Since De Rham cohomology classes in $H^{1}(\mathbb{D}-\{0\} ; \mathbb{R})$ are determined by integration around any loop wrapping once positively 
around the origin, we get ([McL16, Lemma 5.5]):

$$
w_{i}=\frac{1}{2 \pi} \int_{\partial \mathbb{D}}-d^{c}\left(\iota^{*} \rho-f\right) .
$$

Let $(r, \vartheta)$ be the standard polar coordinates on $\mathbb{D}$ and let $\partial_{r}:=\frac{\partial}{\partial r}$ be the unit radial vector field on $\mathbb{D}-\{0\}$. Define

$$
\kappa: \mathbb{D}-\{0\} \longrightarrow \mathbb{R}, \quad \kappa:=d\left(\iota^{*} \rho-f\right)\left(\partial_{r}\right) .
$$

Since $\rho$ is an exhausting function, we have that $\rho(z)-f(z)$ tends to infinity as $|z|$ tends to 0 . Hence there exists $\eta \in(0,1)$ so that

$$
\int_{0}^{2 \pi} \kappa\left(\eta e^{i \vartheta}\right) d \vartheta<0
$$

Therefore

$$
\int_{\{r=\eta\}}-d^{c}\left(\iota^{*} \rho-f\right)=\eta \int_{0}^{2 \pi} \kappa\left(\eta e^{i \vartheta}\right) d \vartheta<0 .
$$

Hence the integral (6.10) is negative which implies that the wrapping number of $\theta$ around $D_{i}$ is negative.

Definition 6.13. Let $W$ be a manifold of dimension $2 n$ with an almost complex structure $J$. The anti-canonical bundle of $(W, J)$ is the complex line bundle $\kappa_{W}^{*}:=\wedge_{\mathbb{C}}^{n}(T W, J)$. Let $\left(\Delta_{j}\right)_{j \in S}$ be a finite collection of transversally intersecting codimension 2 submanifolds. Define $W^{o}:=W-\cup_{j \in S} \Delta_{j}$. Let $\tau:\left.\kappa_{W}^{*}\right|_{W^{o}} \longrightarrow W^{o} \times \mathbb{C}$ be a trivialization of the anti-canonical bundle of $\left(W^{o}, J\right)$. Let $N \subset W$ be a neighborhood of $\cup_{j \in S} \Delta_{j}$ which deformation retracts on to $\cup_{j \in S} \Delta_{j}$ and let $s$ be a smooth section of $\left.\kappa_{W}^{*}\right|_{N}$ transverse to 0 so that $s(x):=\tau^{-1}(x, 1)$ for all $x$ outside a compact subset of $N$. Then $\left[s^{-1}(0)\right]=-\sum_{j} a_{j}\left[\Delta_{j}\right] \in H_{2 n-2}(N ; \mathbb{Z})$ for unique $a_{j} \in \mathbb{Z}, j \in S$. We define the $\tau$-discrepancy of $\Delta_{j}$ to be $a_{j}$ for each $j \in S$. If it is clear that we are using the trivialization $\tau$ (up to isotopy), we will call the $\tau$-discrepancy of $\Delta_{j}$ the discrepancy of $\Delta_{j}$ for each $j \in S$.

The following lemma gives us an example of an SC divisor where the discrepancy of its components are non-negative. Before we state the lemma, we remind the reader of some important notions in algebraic geometry.

Definition 6.14. Let $Y$ be a compact complex manifold of complex dimension $n$. We define the canonical bundle $\kappa_{Y}$ of $Y$ to be the dual of the anti-canonical bundle of $Y$. Let $\pi: \check{Y} \longrightarrow Y$ be a holomorphic map. We define the relative canonical bundle of $\pi$ to be

$$
\kappa_{\widetilde{Y} / Y}:=\kappa_{\widetilde{Y}} \otimes\left(\pi^{*} \kappa_{Y}\right)^{*} .
$$

For any subvariety $F \subset \check{Y}$, we say that $F$ is contracted by $\pi$ if the dimension of the subvariety $\pi(F) \subset Y$ is less than the dimension of $F$.

Recall that a divisor in $Y$ is a formal $\mathbb{Z}$-linear combination $D=\sum_{i=1}^{l} a_{i} D_{i}$ of codimension 1 subvarieties $D_{1}, \cdots, D_{l}$ in $Y$. Such a divisor is effective if $a_{i} \geq 0$ for each $i=1, \cdots, l$. The support $\operatorname{supp}(D)$ of $D$ is the subset $\bigcup_{i \in\{1, \cdots, l\}, a_{i} \neq 0} D_{i}$. The associated homology class $[D]$ is the sum $\sum_{i=1}^{l} a_{i}\left[D_{i}\right] \in H_{2 n-2}(Y ; \mathbb{Z})$ where $\left[D_{i}\right]$ is the fundamental class of $D_{i}$ (See $\left[\right.$ GH94, Chapter 0 , Section 4]). We will define $[D]^{*} \in H^{2}(Y ; \mathbb{Z})$ to be the Poincaré dual of $[D]$. Two divisors are numerically equivalent if they represent the same homology class. The divisor line bundle correspondence gives us a 1-1 correspondence between divisors $E$ and pairs $(L, s)$ where $L$ is a line bundle and $s$ a holomorphic section of $L$ (see [GH94, Chapter 1, 
Section 1$])$. We define $(s)$ to be the divisor associated to $(L, s)$ under the divisor line bundle correspondence.

Under the divisor line bundle correspondence, we have that $[(s)]^{*}=c_{1}(L)$ ([GH94, Chapter 1, Section 1])). Therefore if $s$ and $\check{s}$ are holomorphic sections of two line bundles $L$ and $\check{L}$ then $(s)$ is numerically equivalent to $(\check{s})$ if and only if $c_{1}(L)=c_{1}(\check{L})$.

Lemma 6.15. Let $\pi: \widetilde{X} \longrightarrow X$ be a morphism of smooth projective varieties over $\mathbb{C}$. Let $A \subset X, \widetilde{A} \subset \widetilde{X}$ be Zariski dense affine subvarieties so that

$$
\left.\pi\right|_{\widetilde{A}}: \widetilde{A} \longrightarrow A
$$

is an isomorphism. Suppose $\widetilde{X}-\widetilde{A}$ is a union of transversally intersecting complex hypersurfaces $D_{1}, \cdots, D_{l}$. Let $\tau: \kappa_{X}^{*} \longrightarrow X \times \mathbb{C}$ be a trivialization of the anti-canonical bundle of $X$ and let $\widetilde{\tau}:=\tau \circ\left(\left.\pi\right|_{\widetilde{A}}\right)$ be the induced trivialization of the anti-canonical bundle of $\widetilde{A}$. Then the $\widetilde{\tau}$-discrepancy of $D_{i}$ is non-negative for each $i=1, \cdots, l$.

Proof. Let $\kappa_{\widetilde{X}}$ and $\kappa_{X}$ be the canonical bundles of $\widetilde{X}$ and $X$ respectively and let $\kappa_{\widetilde{X} / X}$ be the relative canonical bundle of $\pi$. The Jacobian of $\pi$ gives us a holomorphic section $s$ of $\kappa_{\tilde{X} / X}$ in the following way: By Equation $(6.11), \kappa_{\widetilde{X} / X}$ is naturally isomorphic to $\operatorname{Hom}\left(\kappa_{\tilde{X}}^{*}, \pi^{*} \kappa_{X}^{*}\right)$ where $\kappa_{\widetilde{X}}^{*}$ and $\kappa_{X}^{*}$ are the anti-canonical bundles of $\tilde{X}$ and $X$ respectively (See Definition 6.13). Under this identification, the section $s$ is given by the map sending $v_{1} \wedge \cdots \wedge v_{1} \in \kappa_{\widetilde{X}}^{*}$ to $D \pi\left(v_{1}\right) \wedge \cdots \wedge D \pi\left(v_{n}\right) \in \pi^{*} \kappa_{X}^{*}$. We have $(s)=\sum_{i=1}^{l} b_{i} F_{i}$ is an effective divisor with the property that $F_{i}$ is contracted by $\pi$ for each $i=1, \cdots, l$. Since (6.12) is an isomorphism, we get that $F_{i} \subset \widetilde{X}-\widetilde{A}$ for each $i=1, \cdots, k$. Hence for each $i=1, \cdots, k$, there exists $j_{i} \in\{1, \cdots, l\}$ so that $F_{i}=D_{j_{i}}$. Hence $(s)=\sum_{i=1}^{l} a_{i} D_{i}$ for some non-negative integers $a_{1}, \cdots, a_{l}$.

Since $\tau$ is a trivialization of the anti-canonical bundle of $X$, we get an induced trivialization $\check{\tau}: \kappa_{X} \longrightarrow X \times \mathbb{C}$ of the canonical bundle (this is the unique trivialization so that $\check{\tau} \otimes \tau$ is the natural trivialization of $\left.\kappa_{X} \otimes \kappa_{X}^{*}\right)$. Let $\sigma: X \longrightarrow \kappa_{X}$ be the unique smooth section satisfying $\check{\tau}(\sigma(x))=(x, 1)$ for each $x \in X$. By (6.11), we have $\kappa_{\widetilde{X}}=\kappa_{\tilde{X} / X} \otimes \pi^{*} \kappa_{X}$ and hence $s \otimes \pi^{*} \sigma$ is a smooth section of $\kappa_{\tilde{X}}$. Let $s^{\prime}$ be a $C^{\infty}$ small perturbation of $s \otimes \pi^{*} \sigma$ which is transverse to 0 and which is equal to $s \otimes \pi^{*} \sigma$ outside a small neighborhood of $\cup_{i=1}^{l} D_{i}$. Then since $\sigma$ is nowhere zero, we get that $\left[\left(s^{\prime}\right)^{-1}(0)\right]$ is homologous to $\sum_{i=1}^{l} a_{i}\left[D_{i}\right]$. Since $k_{\widetilde{X}}^{*}$ is dual to $k_{X}$, this implies that the $\tau$-discrepancy of $D_{i}$ is $a_{i} \geq 0$ for each $i=1, \cdots, l$.

Definition 6.16. Let $\left(W, \omega_{W}\right)$ be a closed symplectic manifold of dimension $2 n$ and let $\left(\Delta_{i}\right)_{i \in S}$ be a symplectic SC divisor in $W$. Define $W^{o}:=W-\cup_{j \in S} \Delta_{j}$. Let $J$ be an $\omega_{W}$-tame almost complex structure and let $\tau$ be a trivialization of the anti-canonical bundle of $\left(W^{o}, J\right)$.

A contact cylinder in $\check{C} \subset W^{o}$ is a contact cylinder as in Definition 2.3 with the symplectic manifold $(M, \omega)$ replaced by $\left(W^{o}, \omega_{W}\right)$. We say that $\check{C}$ is index bounded if it is index bounded in the sense of Definition 5.23 with $(M, \omega)$ replaced by $\left(W^{o}, \omega_{W}\right)$ and where the symplectic trivialization used along each Reeb orbit is induced by $\tau$ and where we consider all Reeb orbits (not just null homologous ones).

Proposition 6.17. Let $\left(\Delta_{i}\right)_{i \in S}$ be a negatively wrapped symplectic $S C$ divisor in a closed symplectic manifold $\left(W, \omega_{W}\right)$ and let $U$ be an open neighborhood of $\cup_{j \in S} \Delta_{j}$ in $W$. Let $W^{o}, J, \tau$ be as in Definition 6.16 above. Let $\rho: W-\cup_{i \in S} \Delta_{i} \longrightarrow \mathbb{R}$ be an exhausting smooth function. Suppose that the $\tau$-discrepancy of $\Delta_{j}$ is non-negative for each $j \in S$. Then there is an index 
bounded contact cylinder $\check{C}$ contained in $U \cap W^{o}$ whose associated Liouville domain contains $W-U$ and so that $\breve{C}$ contains a regular level set of $\rho$.

Proof of Proposition 6.17. Let $w_{j}, a_{j}$ be the wrapping number and $\tau$-discrepancy of $\Delta_{j}$ for each $j \in S$. By [McL12a] [Lemma 5.3 and Lemma 5.14] (or by [FTMZ14, Theorem 2.12]) we can assume, after smoothly deforming $\left(\Delta_{i}\right)_{i \in S}$ through a family of symplectic SC divisors (which doesn't change the symplectomorphism type of the complement $\left(W^{o}, \omega_{W}\right)$ by [McL12a, Lemma 5.15]), that $\left(\Delta_{i}\right)_{i \in I}$ admits a standard tubular neighborhood as in Definition 6.9. Therefore we let $\pi_{I}: U_{I} \longrightarrow \Delta_{I}, I \subset S$ and $r_{i}: U_{i} \longrightarrow \mathbb{R}, i \in S$ be as in Definition 6.9. We can also assume after shrinking the radius $\epsilon$ that this standard tubular neighborhood is contained in $U$. Also the constants $a_{j}, w_{j}$ do not change under isotopy for all $j \in S$, so we can still assume that $w_{j}<0$ and $a_{j} \geq 0$ for all $j \in S$. Let $\dot{\Delta}_{I}:=\Delta_{I}-\cup_{j \in S-I} \Delta_{j}$, $\dot{U}_{I}:=U_{I} \cap W^{o}$ and

$$
\dot{\pi}_{I}: \dot{U}_{I} \longrightarrow \dot{\Delta}_{I}, \quad \dot{\pi}_{I}(x):=\pi_{I}(x)
$$

for all $I \subset S$. The map $\dot{\pi}_{I}$ is a symplectic fibration with fibers symplectomorphic to $\prod_{i \in I} \dot{\mathbb{D}}_{i}(\epsilon)$ where $\dot{\mathbb{D}}_{i}(\epsilon):=\mathbb{D}_{i}(\epsilon)-0$ and whose structure group is given by the natural action of $U(1)^{I}$.

By the proof of [McL16, Lemma 5.18], we can find a smooth function $g: W \longrightarrow \mathbb{R}$ with the property that $\theta+d g$ restricted to any fiber $\prod_{i \in I} \dot{\mathbb{D}}_{i}(\epsilon)$ of $\dot{\pi}_{I}$ is $\sum_{i \in I}\left(\frac{1}{2} r_{i}^{2}+\frac{1}{2 \pi} w_{i}\right) d \vartheta_{i}$ after shrinking $\epsilon$ where $\left(r_{i}, \vartheta_{i}\right)$ are the natural polar coordinates on $\mathbb{D}_{i}(\epsilon)$. Choose $t^{\prime}>0$ so that

$$
e^{t^{\prime}} \in\left(1, \frac{\left(\frac{1}{2} \epsilon\right)^{2}+\frac{1}{\pi} w_{i}}{\epsilon^{2}+\frac{1}{\pi} w_{i}}\right), \quad \forall i \in I .
$$

Choose $\check{\epsilon} \in\left(0, \frac{1}{2} \epsilon\right)$ small enough so that

$$
\cup_{i \in I}\left\{r_{i}^{2}<\left(\check{\epsilon}^{2}+\frac{1}{\pi} w_{i}\right) e^{-t^{\prime}}-\frac{1}{\pi} w_{i}\right\}-\cup_{i \in I}\left\{r_{i}^{2} \leq \check{\epsilon}^{2}\right\}
$$

contains a regular level set of $\rho$. Let $f:\left[0, \epsilon^{2}\right) \longrightarrow \mathbb{R}$ be a smooth function so that $f(x)=1-x$ for all $x \leq \frac{1}{2} \check{\epsilon}^{2},\left.f\right|_{\left[\check{\epsilon}^{2}, \epsilon^{2}\right]}=0, f \geq 0, f^{\prime} \leq 0,\left.f^{\prime}\right|_{\left(0, \check{\epsilon}^{2}\right)}<0$ and $f^{\prime \prime} \geq 0$ (See Figure 17).

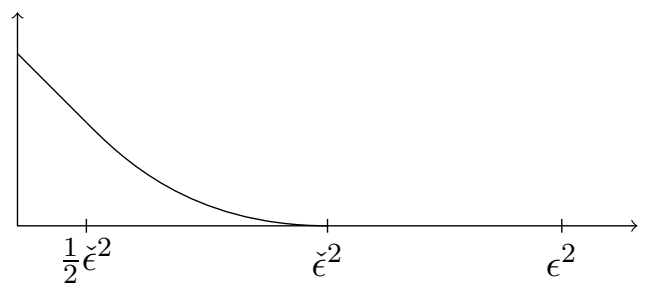

FigURE 17. Graph of $f$.

Define $U_{I}^{o}:=U_{I}-\bigcup_{j \in S-I} U_{j}$ and $\dot{U}_{I}^{o}:=U_{I}^{o} \cap \dot{U}_{I}$. Let $H: W \longrightarrow \mathbb{R}$ be the unique function which satisfies $H(x)=\sum_{i \in I} f\left(r_{i}^{2}\right)$ for all $x \in U_{I}^{o}$ and all $I \subset S$.

Let $\frac{\partial}{\partial r_{i}}$ and $\frac{\partial}{\partial \vartheta_{i}}$ be the unique vector fields on $\dot{U}_{i}$ which are tangent to the fibers of $\dot{\pi}_{I}$ and equal to $\frac{\partial}{\partial r_{i}}$ and $\frac{\partial}{\partial \vartheta_{i}}$ inside the fibers $\dot{\mathbb{D}}_{i}(\epsilon)$ of $\dot{\pi}_{i}$ respectively where $\left(r_{i}, \vartheta_{i}\right)$ are standard 
polar coordinates on $\mathbb{D}_{i}(\epsilon)$. Let $Q_{I} \subset T U_{I}$ be the natural symplectic connection for $\pi_{I}$ as in Definition 6.10. Since the $d \theta$-dual $X_{\theta+d g}$ of $\theta+d g$ inside $\dot{U}_{I}^{o}$ is equal to

$$
\sum_{i \in I}\left(\frac{1}{2} r_{i}+\frac{w_{i}}{2 \pi r_{i}}\right) \frac{\partial}{\partial r_{i}}+E_{I}=\sum_{i \in I}\left(r_{i}^{2}+\frac{w_{i}}{\pi}\right) \frac{\partial}{\partial\left(r_{i}^{2}\right)}+E_{I}
$$

where $E_{I}$ is a vector field tangent to $Q_{I}$ for all $I \subset S$, we get that $d H\left(X_{\theta+d g}\right)>0$ inside $H^{-1}((0, \infty)) \cap \cup_{I \subset S} \dot{U}_{I}$. Hence $C_{\delta}:=H^{-1}(\delta)$ is a compact submanifold of $U$ with contact form $\alpha_{\delta}:=\left.(\theta+d g)\right|_{H^{-1}(\delta)}$ for all sufficiently small $\delta>0$. For each $x \in W^{o}$, let $\psi_{t}(x)$ be the time $t$ flow of $x$ along $-X_{\theta+d g}$ for all $t$ (when defined). Since the region (6.14) contains a regular level set of $\rho$, we have by Equation (6.13) that the contact cylinder with image

$$
\check{C}_{\delta}:=\bigcup_{t \in\left[0, t^{\prime}\right]} \psi_{t}\left(C_{\delta}\right)
$$

contains a regular level set of $\rho$ for all sufficiently small $\delta>0$. Equation (6.13) also ensures that this contact cylinder is contained in $U$. To finish our lemma we will now show that the contact cylinder $\check{C}_{\delta}$ is index bounded for all $\delta>0$ small enough by computing the Conley-Zehnder indices of the Reeb orbits of $\alpha_{\delta}$ for $\delta>0$ small enough.

Let $R_{\delta}$ be the Reeb vector field of $\alpha_{\delta}$. Inside $\dot{U}_{I}^{o}$, we have that $X_{H}=2 \sum_{i \in I} f^{\prime}\left(r_{i}^{2}\right) \frac{\partial}{\partial \vartheta i}$. Define

$$
b: \dot{U}_{I}^{o} \longrightarrow \mathbb{R}, \quad b:=\sum_{i \in I} f^{\prime}\left(r_{i}^{2}\right)\left(r_{i}^{2}+\frac{w_{i}}{\pi}\right) .
$$

Then

$$
R_{\delta}=\frac{1}{(\theta+d g)\left(X_{H}\right)} X_{H}=\frac{2}{b} \sum_{i \in I} f^{\prime}\left(r_{i}^{2}\right) \frac{\partial}{\partial \vartheta i} .
$$

In particular, a Reeb orbit which starts inside $\dot{U}_{I}^{o} \cap H^{-1}(\delta)$, stays inside $\dot{U}_{I}^{o} \cap H^{-1}(\delta)$ and all such Reeb orbits are contained in fibers of $\left.\dot{\pi}_{I}\right|_{H^{-1}(\delta)}$. We will show that the Conley-Zehnder index of every Reeb orbit of $\alpha_{\delta}$ is a bounded above by a linear function of its length whose slope is negative when $\delta>0$ is sufficiently small. This will be sufficient for us to show that $\check{C}_{\delta}$ is index bounded for all $\delta>0$ sufficiently small.

Now let $\zeta: \mathbb{R} / \lambda \mathbb{Z} \longrightarrow H^{-1}(\delta)$ be a Reeb orbit of $\alpha_{\delta}$ of length $\lambda$. Now $\zeta$ is contained inside $\dot{\pi}_{I}^{-1}(q)$ for some $q \in \Delta_{I}-\cup_{j \in S-I} U_{j}$ and $I \subset S$ and so there exists a smooth map $\check{\zeta}: \mathbb{D} \longrightarrow \pi_{I}^{-1}(q)$ from the closed unit disk $\mathbb{D} \subset \mathbb{C}$ so that $\check{\zeta}\left(e^{2 \pi i t}\right)=\zeta(\lambda t)$ for all $t \in[0,1]$. Let $d_{i} \in \mathbb{Z}$ be the intersection number of $\check{\zeta}$ with $\Delta_{i} \cap \pi_{I}^{-1}(q)$ inside $\pi^{-1}(q)$ for each $i \in I$. Define $C Z(\zeta)$ to be the Conley-Zehnder index of $\zeta$ inside $W^{o}$ and let $C Z(\check{\zeta})$ to be the ConleyZehnder index of $\zeta$ inside a small neighborhood of $\pi_{I}^{-1}(q)$ (I.e. we think of a portion of our contact cylinder containing this orbit as a contact cylinder in a neighborhood of $\left.\pi_{I}^{-1}(q)\right)$. By (CZ1), (CZ2) and (CZ3) we have

$$
C Z(\zeta)=C Z(\check{\zeta})+2 \sum_{i \in I} d_{i} a_{i}
$$

By Equation (6.15) combined with the fact that $f^{\prime}\left(f^{-1}(x)\right)<0$ for all small $x>0$, we see that $d_{i}<0$ for all $i \in I$ and that the length of the Reeb orbit $\zeta$ is bounded below by a positive constant times $-\sum_{i \in I} d_{i}$. Therefore it is sufficient for us to show that $C Z(\zeta)$ is less than or equal to some fixed linear function of $\sum_{i \in I} d_{i}$ of positive slope.

Let $T:=T\left(\pi_{I}^{-1}(q)\right) \subset T W$ be the tangent space of the fiber containing $\zeta$ and let $T^{\perp} \subset T W$ be the set of vectors which are $\omega_{W}$ orthogonal to $T$. Let $x_{1}, y_{1}, \cdots, x_{|I|}, y_{|I|}$ be symplectic 
coordinates of $\pi_{I}^{-1}(q)$ coming from a $U(1)^{|I|}$ trivialization of this fiber and let $J$ be the natural complex structure coming from this trivialization. These coordinates induce a symplectic trivialization $\tau_{T}: \zeta^{*} T \longrightarrow \mathbb{R} / \lambda \mathbb{Z} \times \mathbb{C}^{|I|}$ of $\zeta^{*} T$. Define $K_{\delta}:=\operatorname{ker}\left(\alpha_{\delta}\right) \cap T \subset T$ and let $\left.K_{\delta}^{\perp} \subset T\right|_{\dot{\pi}_{I}^{-1}(q)}$ be the symplectic vector subspace of $T$ consisting of vectors which are $\left.\omega_{W}\right|_{T^{-}}$ orthogonal to $K_{\delta}$. Since $R_{\delta}$ is contained in $K_{\delta}^{\perp}$ and $K_{\delta}^{\perp}$ is a two dimensional vector bundle, there is a unique, up to homotopy, trivialization $\tau_{R_{\delta}}: K_{\delta}^{\perp} \longrightarrow \dot{\pi}_{I}^{-1}(q) \times \mathbb{C}$ of $K_{\delta}^{\perp}$ which maps $R_{\delta}$ to the constant section whose value is $i \in \mathbb{C}$. Hence there is a symplectic trivialization $\check{\tau}: \zeta^{*} K_{\delta} \longrightarrow \mathbb{R} / \lambda \mathbb{Z} \times \mathbb{C}^{|I|-1}$ so that $\check{\tau} \oplus \tau_{R_{\delta}}$ gives us a trivialization of $\zeta^{*} T=\zeta^{*}\left(K_{\delta} \oplus K_{\delta}^{\perp}\right)$ homotopic to $\tau_{T}$.

Let $\tau_{T^{\perp}}: \zeta^{*} T^{\perp} \longrightarrow \mathbb{R} / \lambda \mathbb{Z} \times \mathbb{C}^{n-|I|}$ be a trivialization of $\zeta^{*} T^{\perp}$ which is a restriction of a trivialization of $T^{\perp}$ (such a trivialization is unique up to homotopy since $\pi_{I}^{-1}(q)$ is contractible). Then

$$
\check{\tau} \oplus \tau_{T^{\perp}}: \zeta^{*}\left(K_{\delta} \oplus T^{\perp}\right)=\zeta^{*} \operatorname{ker}\left(\alpha_{\delta}\right) \longrightarrow \mathbb{R} / \lambda \mathbb{Z} \times \mathbb{C}^{n-1}
$$

is a trivialization of $\zeta^{*} \operatorname{ker}\left(\alpha_{\delta}\right)$. This is the trivialization we need in order to compute $C Z(\check{\zeta})$ since the trivialization $\tau_{R_{\delta}} \oplus \check{\tau} \oplus \tau_{T^{\perp}}$ of $\zeta^{*} T W$ extends over the disk $\check{\zeta}^{*} T W$ after identifying the boundary of $\partial \mathbb{D}$ with $\mathbb{R} / \lambda \mathbb{Z}$ in the natural way as explained earlier.

If $\phi_{t}^{\delta}: H^{-1}(\delta) \longrightarrow H^{-1}(\delta)$ is the time $t$ flow of $R_{\delta}$ then its linearization $D \phi_{t}:\left.\operatorname{ker}\left(\alpha_{\delta}\right)\right|_{\zeta(0)} \longrightarrow$ $\left.\operatorname{ker}\left(\alpha_{\delta}\right)\right|_{\zeta(t)}$ gives us a family of symplectic matrices $\left(A_{t}\right)_{t \in[0, \lambda]}$ with respect to the trivialization (6.16). Equation (6.15) tells us that $D \phi_{t}^{\delta}$ respects the splitting $\zeta^{*} \operatorname{ker}\left(\alpha_{\delta}\right)=\zeta^{*} K_{\delta} \oplus \zeta^{*} T^{\perp}$ and hence $A_{t}=B_{t} \oplus C_{t}$ for some family of matrices $\left(B_{t}\right)_{t \in[0, \lambda]}$ and $\left(C_{t}\right)_{t \in[0, \lambda]}$ with respect to the trivializations of $\zeta^{*} K_{\delta}$ and $\zeta^{*} T^{\perp}$. Also Equation (6.15) tells us that $C_{t}=\mathrm{id}$ for all $t \in[0, \lambda]$ after homotoping $\tau_{T^{\perp}}$ appropriately. By (CZ6), we have that $C Z\left(\left(C_{t}\right)_{t \in[0, \lambda]}\right)=0$. Hence by (CZ2), all we need to do is compute the Conley-Zehnder index of $\left(B_{t}\right)_{t \in[0, \lambda]}$.

In order to compute $C Z\left(\left(B_{t}\right)_{t \in[0, \lambda]}\right)$ we will compute the Conley-Zehnder index with respect to an alternative trivialization of $K_{\delta}$ and relate it to the trivialization $\check{\tau}$ above. Choose a total ordering on $I$. This means we have a natural identification $I=\{1, \cdots, l\}$. Define

$$
\begin{gathered}
\check{R}_{i}:=\left(2 r_{l} f^{\prime}\left(r_{l}^{2}\right)\right) \frac{\partial}{\partial r_{i}}-\left(2 r_{i} f^{\prime}\left(r_{i}^{2}\right)\right) \frac{\partial}{\partial r_{l}} \\
\Theta_{i}:=\left(\frac{1}{2} r_{l}^{2}+\frac{1}{2 \pi} w_{l}\right) \frac{\partial}{\partial \vartheta_{i}}-\left(\frac{1}{2} r_{i}^{2}+\frac{1}{2 \pi} w_{i}\right) \frac{\partial}{\partial \vartheta_{l}}
\end{gathered}
$$

for all $i \in\{1, \cdots, l-1\}$. Let $L$ be the span of $\Theta_{1}, \cdots, \Theta_{l-1}$ and $L^{\perp}$ the span of $\check{R}_{1}, \cdots, \check{R}_{l-1}$. Since $K_{\delta}=\operatorname{ker}\left(\alpha_{\delta}\right)=\operatorname{ker}(\theta+d g) \cap \operatorname{ker}(d H)$, we get that $\check{R}_{1}, \Theta_{1}, \cdots, \check{R}_{l-1}, \Theta_{l-1}$ is a basis for $K_{\delta}$. The problem with this basis is that it is not a symplectic basis. However since $L$ and $L^{\perp}$ are Lagrangian, there is a new basis $R_{1}, \cdots, R_{l-1}$ of $L^{\perp}$ so that $R_{1}, \Theta_{1}, \cdots, R_{l-1}, \Theta_{l-1}$ is a symplectic basis of $K_{\delta}$. Since the Poisson bracket of $R_{\delta}$ with each of $\Theta_{1}, \cdots, \Theta_{l-1}$ is zero by Equation (6.15), we have that the flow of $R_{\delta}$ sends $\Theta_{i}$ to $\Theta_{i}$ for each $i=1, \cdots, l-1$. The linearization $\left.D \phi_{t}^{\delta}\right|_{K_{\delta}}:\left.\left.K_{\delta}\right|_{\zeta(0)} \longrightarrow K_{\delta}\right|_{\zeta(t)}$ of the Reeb flow $\phi_{t}^{\delta}$ of $R_{\delta}$ is a family of symplectic matrices $\left(W_{t}\right)_{t \in[0, \lambda]}$ with respect to the symplectic basis $R_{1}, \Theta_{1}, \cdots, R_{l-1}, \Theta_{l-1}$. Since $W_{t}(x)=x$ on $L$ with respect to this basis for all $t \in[0, \lambda]$, we have by Lemma 6.8 that $C Z\left(\left(W_{t}\right)_{t \in[0, \lambda]}\right) \in[-2 l-2,2 l+2] \subset[-2 n+2,2 n-2]$.

Let $\widehat{\tau}: \zeta^{*} K_{\delta} \longrightarrow \mathbb{R} / \lambda \mathbb{Z} \times \mathbb{C}^{l-1}$ be the trivialization of $\zeta^{*} K_{\delta}$ induced by the symplectic basis $R_{1}, \Theta_{1}, \cdots, R_{l-1}, \Theta_{l-1}$. Then $\widehat{\tau} \oplus \tau_{R_{\delta}}$ is isotopic to the trivialization induced by the symplectic basis

$$
\frac{1}{r_{1}} \frac{\partial}{\partial r_{1}}, \frac{\partial}{\partial \theta_{1}}, \cdots, \frac{1}{r_{l}} \frac{\partial}{\partial r_{l}}, \frac{\partial}{\partial \theta_{l}}
$$


Hence the bundle automorphism $\check{\tau} \circ \widehat{\tau}^{-1}$ gives us a family of symplectic matrices parameterized by $[0, \lambda]$ whose Conley-Zehnder index is $2 \sum_{i \in I} d_{i}$. Hence by (CZ1), (CZ2) and (CZ3),

$$
C Z\left(\left(B_{t}\right)_{t \in[0, \lambda]}\right)=C Z\left(\left(W_{t}\right)_{t \in[0, \lambda]}\right)+2 \sum_{i \in I} d_{i} .
$$

Hence by (CZ2), we get that

$$
C Z\left(\left(A_{t}\right)_{t \in[0, \lambda]}\right)=C Z\left(\left(B_{t}\right)_{t \in[0, \lambda]}\right)+C Z\left(\left(C_{t}\right)_{t \in[0, \lambda]}=2 \sum_{i \in I} d_{i}+C Z\left(\left(W_{t}\right)_{t \in[0, \lambda]}\right) .\right.
$$

Hence

$$
C Z(\zeta)=2 \sum_{i \in I}\left(a_{i}+1\right) d_{i}+C Z\left(\left(W_{t}\right)_{t \in[0, \lambda]}\right)
$$

which is bounded above by a linear function of length of $\zeta$ with negative slope since we have $C Z\left(\left(W_{t}\right)_{t \in[0,1]}\right) \in[-2 n+2,2 n-2]$. Therefore $\check{C}:=\check{C}_{\delta}$ is index bounded. The associated Liouville domain of $\check{C}$ is contained in $W^{o}$ and contains $K$ since its complement is contained in $\cup_{i \in S} U_{i}$.

Proof of Proposition 6.7. By [Hir64], we can blow up $X$ along smooth subvarieties above $X-A$ giving us a variety $\widetilde{X}$ so that the preimage of $X-A$ is a smooth normal crossing variety. Let $B l: \widetilde{X} \longrightarrow X$ be the blowdown map. Choose an algebraic plurisubharmonic function $\widetilde{\rho}: A \longrightarrow \mathbb{R}$ so that $-d d^{c}\left(B l^{*}(\widetilde{\rho})\right)$ extends to a Kähler form $\omega_{\widetilde{X}}$ on $\widetilde{X}$ (I.e. $\widetilde{\rho}=-d d^{c} \log (|s|)$ for some appropriate section $s$ of an ample line bundle on $\widetilde{X}$ with an appropriate Hermitian metric). By Corollary 6.6 there are constants $0<\delta \ll T \ll 1$ and a compact set $Q \subset A$ containing $K$ and a plurisubharmonic function $\widehat{\rho}$ on $A$ satisfying

(1) $\left.\widehat{\rho}\right|_{Q}=\left.\rho\right|_{Q}$,

(2) $\widehat{\rho}=\rho+\delta \widetilde{\rho}$ outside a large compact set and

(3) for all $x \in A$, the time $T$ flow of $x$ along $-\nabla_{\widehat{\rho}} \widehat{\rho}$ is contained in $Q$ and is disjoint from $K$ if, in addition, $x \notin Q$.

Let $\phi_{T}: A \longrightarrow A$ be the time $T$ flow of $-\nabla_{\widehat{\rho}} \widehat{\rho}$. By Lemmas 6.12 and 6.15 combined with Proposition 6.17, we can find an index bounded contact cylinder $\check{C}_{1} \subset B l^{-1}(A)$ with respect to the symplectic form $B l^{*} \omega_{X}+\delta \omega_{\widetilde{X}}$ which is disjoint from $Q$ and whose associated Liouville domain contains $Q$ and which contains a regular level set $\widehat{\rho}^{-1}(C)$ for some $C \in \mathbb{R}$. Then $\check{C}:=\phi_{T}\left(B l\left(\check{C}_{1}\right)\right) \subset A$ is an index bounded contact cylinder with respect to $\omega_{X}$ since $\left.\omega_{X}\right|_{Q}=\left.\omega_{\tilde{X}}\right|_{Q}$ and since both of these symplectic forms are Kähler. Also the Liouville domain associated to $\check{C}$ contains $K$ and $\check{C}$ contains $C_{0}:=\phi_{T}\left(B l\left(\widehat{\rho}^{-1}(C)\right)\right)$ which is a hypersurface transverse to $\nabla_{\rho} \rho$ bounding a region containing $D$. By a Moser argument ([MS98, Exercise $3.36])$, we can enlarge $\check{C}$ slightly so that it contains a contact cylinder $\check{C}_{0}:=\left[1-\epsilon_{0}, 1+\epsilon_{0}\right] \times C_{0}$ where $\{0\} \times C_{0}=C_{0}$. The associated Liouville domain $D_{0}$ contains $D$ and $-\left.d^{c} \rho\right|_{D_{0}}$ is a Liouville form associated to $\check{C}_{0}$.

6.3. Divisors are Stably Displaceable. We recall from Section 1.3 that $(M, \omega)$ is a compact symplectic manifold. However, the results of this section also work when $M$ is noncompact. We also do not require that $(M, \omega)$ satisfy any other conditions, such as the Chern class condition $c_{1}(M, \omega)=0$.

Definition 6.18. A partly stratified symplectic subset $S$ of $(M, \omega)$ is a subset equal to a disjoint union of subsets $S_{1}, \cdots, S_{l}$ of $M$ so that for each $j \in\{1, \cdots, l\}$,

- $\cup_{i \leq j} S_{i}$ is a compact subset of $M$ and 
- $S_{j}$ is a proper codimension $\geq 2$ symplectic submanifold of $M-\cup_{i<j} S_{i}$ without boundary.

We call the subsets $S_{1}, \cdots, S_{l}$ the strata of $S$.

Example 6.19. [Whi65, Theorem 19.2]. If $M$ is a Kähler manifold then any compact codimension $\geq 1$ subvariety of $M$ is a partly stratified symplectic subset.

Proposition 6.20. Any partly stratified symplectic subset is stably displaceable as in Definition 5.11.

We have the following immediate corollary:

Corollary 6.21. Any compact positive codimension subvariety of a Kähler manifold is stably displaceable.

The rest of this subsection is devoted to the proof of Proposition 6.20. Throughout this subsection we will let $(\sigma, \tau)$ be the natural Darboux coordinates on $T^{*} \mathbb{T}=\mathbb{R} \times \mathbb{T}$ where $\sigma$ is the projection map to $\mathbb{R}$ and $\tau$ is the projection map to $\mathbb{T}$. We will also let $(\check{M}, \check{\omega})$ be the symplectic manifold $\left(M \times T^{*} \mathbb{T}, \omega+d \sigma \wedge d \tau\right)$.

Let us first give a sketch of the proof of Proposition 6.20. It would be nice if one could displace $S \times \mathbb{T}$ using the symplectic vector field $V:=\frac{\partial}{\partial \sigma}$. However this is not a Hamiltonian vector field. What we wish to do is to subtract another time dependent symplectic vector field $V_{t}, t \in \mathbb{R}$ from $V$ so that $V-V_{t}$ is Hamiltonian and so that the time $T$ flow of $S \times \mathbb{T}$ along $V-V_{t}$ is a bounded distance from the time $T$ flow of $S \times \mathbb{T}$ along $V$ for some large $T \in \mathbb{R}$. To do this, we first 'curl up' $S \times T^{*} \mathbb{T}$ into a small neighborhood of $S \times \mathbb{T}$. In other words, we find an appropriate symplectic embedding $\iota$ of a neighborhood $N$ of $S \times T^{*} \mathbb{T}$ into a relatively compact subset of $M \times \mathbb{T}^{*} \mathbb{T}$. This is done via an explicit embedding technique from [EM02, Lemma 12.1.2]. We then define $V_{t}$ to be an appropriate extension of $\left(\phi_{t}^{V}\right)_{*}\left(\iota_{*} V\right), t \in \mathbb{R}$ to $\check{M}$ where $\phi_{t}^{V}, t \in \mathbb{R}$ is the flow of $V$ (See Figure 18). Then $V-V_{t}$ is the Hamiltonian vector field displacing $S \times \mathbb{T}$ in $M \times T^{*} \mathbb{T}$. This completes the sketch of the proof.

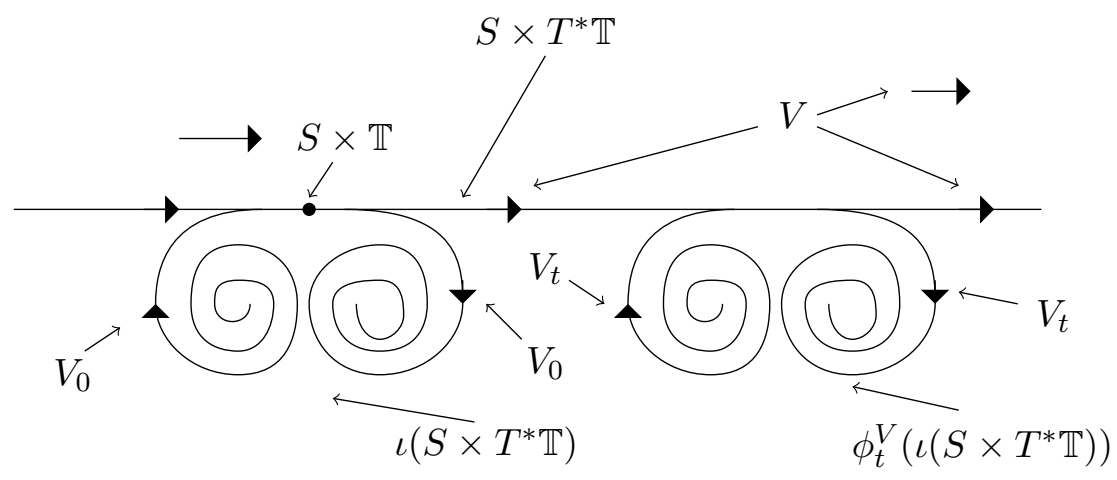

FiguRE 18. Picture of $V$ and $V_{t}, t \in \mathbb{R}$.

If $S$ has only one stratum (I.e. $S$ is a symplectic submanifold) then the methods of [LS94] and [Gür08, Section 4.3] can be used to displace $S \times \mathbb{T}$ instead. However it is hard to see how these methods could be used when $S$ has singularities. This is due to the fact that near certain singular points $p$ of $S$ (such as normal crossing points), there is no vector field 
that can infinitesimally displace a neighborhood of $p$ in $S$ from $S$. Also there are examples which seem difficult to infinitesimally displace. For instance a $C^{\infty}$ generic Hamiltonian on $\mathbb{C P}^{2} \times T^{*} \mathbb{T}$ cannot displace $(A \cup B) \times \mathbb{T}$ by an infinitesimally small amount where $A, B \subset \mathbb{C P}^{2}$ are distinct complex lines.

Before we prove Proposition 6.20, we need some preliminary definitions and lemmas. We will first provide a criterion for Hamiltonian displacement in terms of 'curled up' symplectic embeddings.

Definition 6.22. For each $\nu>0$, define

$$
\check{M}_{<\nu}:=\{(x,(\sigma, \tau)) \in \check{M}:|\sigma|<\nu\} .
$$

Let $Q \subset M$ be a subset. A weak curled up embedding of $Q \times T^{*} \mathbb{T}$ consists of a neighborhood $N$ of $Q \times T^{*} \mathbb{T}$ in $\check{M}$ and a symplectic embedding $\iota: N \hookrightarrow \check{M}_{<1}$ so that $\iota(y)=y$ for all $y$ in a neighborhood of $N \cap(M \times \mathbb{T}) \subset N$. A curled up embedding of $Q \times T^{*} \mathbb{T}$ is a weak curled up embedding as above with the additional property that the map $H^{1}(N ; \mathbb{R}) \longrightarrow$ $H^{1}(N \cap(M \times \mathbb{T}) ; \mathbb{R})$ induced by the inclusion map is injective.

Lemma 6.23. Let $Q \subset M$ be a compact subset of $M$ so that $Q \times T^{*} \mathbb{T}$ admits a curled up embedding. Then $Q$ is stably displaceable.

Proof. Let $\iota, N$ be as in Definition 6.22 above. Let $V:=\frac{\partial}{\partial \sigma}$. We wish to construct $V_{t}, t \in \mathbb{R}$ as described above. Define $\widetilde{V}:=\iota_{*}(V)$. Let $\phi_{t}^{V}$ be the time $t$ flow of $V$ for each $t \in \mathbb{R}$ and similarly define $\phi_{t}^{\widetilde{V}}$ (when defined). Since $\iota(y)=y$ for all $y \in \check{M}$ near $N \cap(M \times \mathbb{T}$ ), we get that the closed 1-form $\beta(-):=\check{\omega}\left(\left.V\right|_{\iota(N)}-\widetilde{V},-\right)$ vanishes near $N \cap(M \times \mathbb{T})$. Combining this with the fact that the map $H^{1}(N ; \mathbb{R}) \longrightarrow H^{1}(N \cap(M \times \mathbb{T}) ; \mathbb{R})$ induced by the inclusion map is injective, we have that $\beta$ is exact. Hence $\left.V\right|_{\iota(N)}-\widetilde{V}$ is a Hamiltonian vector field on $\iota(N) \subset \check{M}$. Let $\check{H}: \iota(N) \longrightarrow \mathbb{R}$ be the corresponding Hamiltonian. Let $\rho: \iota(N) \longrightarrow[0,1]$ be a smooth compactly supported function whose restriction to a small neighborhood of the relatively compact set $\widetilde{Q}:=\iota\left(\left(Q \times T^{*} \mathbb{T}\right) \cap \check{M}_{<3}\right)$ is 1 and define

$$
\widetilde{H}: \check{M} \longrightarrow \mathbb{R}, \quad \widetilde{H}(y):= \begin{cases}\rho(y) \check{H}(y) & \text { if } y \in \iota(N) \\ 0 & \text { otherwise. }\end{cases}
$$

Define $H_{t}:=\left(\phi_{t}^{V}\right)_{*}(\widetilde{H})$ for each $t \in \mathbb{R}$. Note that the vector field $V_{t}$ in the proof sketch above is just $V-X_{H_{t}}$. Our claim is that $H:=\left(H_{t}\right)_{t \in \mathbb{R}}$ is the Hamiltonian which displaces $Q$. Let $\phi_{t}^{H}$ be the time $t$ flow of $H$ for each $t \in \mathbb{R}$. Near $\phi_{t}^{V}(\widetilde{Q})$, we have

$$
X_{H_{t}}=\left(\phi_{t}^{V}\right)_{*}\left(X_{\widetilde{H}}\right)=\left(\phi_{t}^{V}\right)_{*}(V-\widetilde{V})=V+\left(\phi_{t}^{V}\right)_{*}(-\widetilde{V})
$$

for all $t \in \mathbb{R}$. Hence for each $y \in Q \times \mathbb{T}, \phi_{t}^{H}(y)=\phi_{t}^{V}\left(\phi_{t}^{-\widetilde{V}}(y)\right)$ for all $y \in Q \times \mathbb{T}$ and $t \in[0,3]$. Combining this with the fact that $\phi_{t}^{V}\left(\phi_{t}^{-\widetilde{V}}(y)\right) \in \phi_{t}^{V}\left(\check{M}_{<1}\right)$ for all $y \in Q \times \mathbb{T}$, and $\phi_{3}^{V}\left(\check{M}_{<1}\right) \cap \check{M}_{<1}=\emptyset$ we get $\phi_{3}^{H}(Q \times \mathbb{T}) \cap(Q \times \mathbb{T})=\emptyset$. Hence $Q$ is stably displaceable.

Definition 6.24. Let $A \subset M \times \mathbb{T}$ be a subset. For any function $f: A \longrightarrow \mathbb{R} \cup\{\infty\}$, define

$$
\left.\check{M}_{<f}\right|_{A}:=\left\{(x,(\sigma, \tau)) \in A \times T^{*} \mathbb{T}:|\sigma|<f(x, \tau)\right\} \subset \check{M} .
$$

Let $N \subset \check{M}$ be an open subset containing $A$. Define

$$
f_{N}: A \longrightarrow \mathbb{R} \cup\{\infty\}, \quad f_{N}(a):=\sup \{\sigma>0:\{a\} \times[-\sigma, \sigma] \subset N\} .
$$

See Figure 19. We define

$$
\left.\check{M}_{<N}\right|_{A}:=\left.\check{M}_{<f_{N}}\right|_{A}
$$


If $A=M \times \mathbb{T}$, we define

$$
\check{M}_{<N}:=\check{M}_{<N} \mid A .
$$

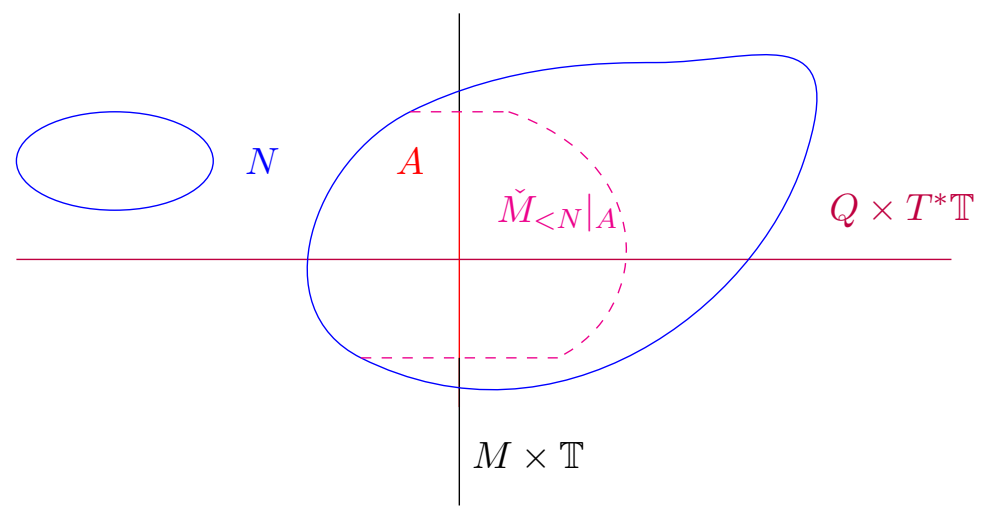

Figure 19. Picture of $\left.\check{M}_{<N}\right|_{A}$.

Lemma 6.25. The function $f_{N}$ from Definition 6.24 is lower semi-continuous. Hence $\left.\check{M}_{<N}\right|_{A}$ is an open subset of $\check{M}$ if $A \subset M \times \mathbb{T}$ is open.

Proof of Lemma 6.25. Let $a \in A$ and let $\left(a_{i}\right)_{i \in \mathbb{N}}$ be a sequence of points in $A$ converging to $a$. Let $\sigma>0$ satisfy $\sigma<f_{N}(a)$. Since $N \subset M$ is an open subset and since $\{a\} \times[-\sigma, \sigma]$ is compact, we have that $\left\{a_{j}\right\} \times[-\sigma, \sigma] \subset N$ for all $j$ sufficiently large. Hence $\sigma<f_{N}\left(a_{j}\right)$ for all $j$ sufficiently large. Hence

$$
\liminf _{j \rightarrow \infty} f_{N}\left(a_{j}\right) \geq \liminf _{\sigma<f_{N}(a)} \sigma=f_{N}(a) .
$$

Hence $f_{N}$ is lower semi-continuous.

Lemma 6.26. Let $Q \subset M$ be a compact subset of $M$ so that $Q \times T^{*} \mathbb{T}$ admits a weak curled up embedding. Then $Q \times T^{*} \mathbb{T}$ admits a curled up embedding.

Proof. Let $\iota: N \hookrightarrow \check{M}_{<1}$, be a weak curled up embedding of $Q \times T^{*} \mathbb{T}$. The claim is that $\left.\iota\right|_{\left(\check{M}_{<N}\right)}$ is a curled up embedding. To prove this, it is sufficient to show that $\check{M}_{<N}$ deformation retracts onto $E$. This deformation retraction is given by

$$
\Phi_{t}: \check{M}_{<N} \longrightarrow \check{M}_{<N}, \quad \Phi(x,(\sigma, \tau)):=(x,((1-t) \sigma, \tau)), \quad t \in[0,1] .
$$

We need the following Moser lemma in order to construct appropriate weak curled up embeddings of stratified symplectic subsets. The proof of this lemma is a slight modification of [MS98, Lemma 3.14].

Lemma 6.27. Let $\left(W, \omega_{W}\right),\left(\check{W}, \omega_{\breve{W}}\right)$ be symplectic manifolds, let $Q \subset W$ be a symplectic submanifold and let $U, N \subset W$ open sets satisfying $\bar{U} \subset N$. Let $\iota_{N}: N \hookrightarrow \check{W}, \iota_{Q}: Q \hookrightarrow \check{W}$ be symplectic embeddings so that

(1) $\iota_{N}$ is a codimension 0 symplectic embedding,

(2) $\left.\iota_{N}\right|_{N \cap Q}=\left.\iota_{Q}\right|_{N \cap Q}$ and 
(3) the pullback via $\iota_{Q}$ of the normal bundle of $\iota_{Q}(Q)$ is isomorphic as a symplectic vector bundle to the normal bundle of $Q$ in $W$ and this isomorphism coincides with the isomorphism induced by $\iota_{N}$ along $N \cap Q$.

Then there is a neighborhood $V \subset W$ of $Q$ and a codimension 0 symplectic embedding $\iota$ : $U \cup V \hookrightarrow \check{W}$ so that $\iota_{U}=\iota_{U}$ and $\iota_{Q}=\iota_{Q}$ (See Figure 20).

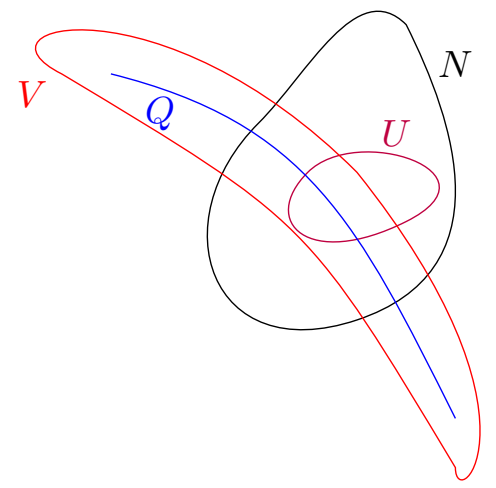

Figure 20. Embedding of $U \cup V$ into $\check{W}$.

Lemma 3.1 in [MS98] requires that $Q$ be compact. However this is really not needed.

Proof of Lemma 6.2\%. Choose complete metrics $g$ and $\check{g}$ on $W$ and $\check{W}$ respectively so that $\iota_{N}^{*} \check{g}=g$ near $\bar{U}$ and let $N Q$ be the symplectic normal bundle of $Q$ in $W$ and let $E \longrightarrow \iota_{Q}(Q)$ be the symplectic normal bundle of $\iota_{Q}(Q)$ in $\check{W}$. Let $\exp _{W}: N Q \longrightarrow W$ and $\exp _{\breve{W}}: E \longrightarrow \breve{W}$ be the exponential maps using $g$ and $\check{g}$ respectively. Let $\phi: N Q \longrightarrow \iota_{Q}^{*} E$ be the isomorphism described in (3) above. Choose small tubular neighborhoods $A \subset N Q$ and $B \subset E$ of the zero section so that $\left.\exp _{W}\right|_{A}$ and $\left.\exp _{\breve{W}}\right|_{B}$ are smooth embeddings and so that $\phi(A)=B$. We have a smooth family of symplectic forms

$$
\omega_{t}:=(1-t)\left(\left(\exp _{\breve{W}}\right)_{*} \phi_{*} \exp _{W}^{*} \omega_{W}\right)+t \omega_{\breve{W}}, \quad t \in[0,1]
$$

on $\widetilde{B}:=\exp _{\breve{W}}(B)$ after shrinking $A$ and $B$. Now $\omega_{t}$ is equal to $\omega_{\breve{W}}$ along $\left.T \breve{W}\right|_{\iota_{Q}(Q)}$ and also along some neighborhood $U^{\prime} \subset \widetilde{B}$ of $\bar{U} \cap \widetilde{B}$ for each $t \in[0,1]$. After shrinking $U^{\prime}, A$ and $B$, we can assume that $U^{\prime}$ deformation retracts onto $U^{\prime} \cap \iota_{Q}(Q)$. Hence we can find a smooth family of 1-forms $\sigma_{t} \in \Omega^{1}(\widetilde{B}), t \in[0,1]$ satisfying

- $\frac{d}{d t} \omega_{t}=d \sigma_{t}$ and

- $\sigma_{t}(v)=0$ for each $v \in T_{x} \widetilde{B}, x \in U^{\prime} \cup \iota_{Q}(Q)$ and $t \in[0,1]$

after shrinking $U^{\prime}$ slightly again. Let $\left(X_{t}\right)_{t \in[0,1]}$ be a smooth family of vector fields satisfying $\sigma_{t}+\iota_{X_{t}} \omega_{t}=0$ for each $t \in[0,1]$. Let $\psi_{t}$ be the time $t$ flow of $\left(X_{t}\right)_{t \in[0,1]}$ for each $t \in[0,1]$ (if it exists). For each $x \in \iota_{Q}(Q)$, we have that $X_{t}=0$ at $x$ since $\sigma_{t}=0$ at $x$. Therefore for each $x \in \iota_{Q}(Q)$, there is a small neighborhood $V_{x} \subset \widetilde{B}$ of $x$ so that the time $t$ flow $\psi_{t}(y)$ is well defined for each $y \in V_{x}$ and $t \in[0,1]$. Hence the time $t$ flow $\psi_{t}(y)$ is well defined for 
each $y \in V:=\cup_{x \in Q} V_{x}$ and each $t \in[0,1]$. Define

$$
\iota: U \cup V \hookrightarrow \check{W}, \quad \iota(x):= \begin{cases}\iota_{N}(x) & \text { if } x \in U \\ \psi_{1}\left(\exp _{\breve{W}}\left(\phi\left(\exp _{W}^{-1}(x)\right)\right)\right. & \text { if } x \in V .\end{cases}
$$

This is a symplectic embedding. Also since $\psi_{1}(x)=x$ for all $x \in(\bar{U} \cap \widetilde{B}) \cup \iota_{Q}(Q)$ we have $\left.\iota\right|_{U}=\iota_{U}$ and $\left.\iota\right|_{Q}=\iota_{Q}$.

The proof of the following lemma is identical to the proof of [EM02, Lemma 12.1.2] and so we will omit it. However, we will give an idea of the proof since it will be a crucial ingredient in the proof of Proposition 6.20 below.

Lemma 6.28. Let $Q$ be a symplectic submanifold of a symplectic manifold $\left(W, \omega_{W}\right)$ of positive codimension and let $U, N \subset Q$ be open sets so that $\bar{U} \subset N$. Let $\alpha_{t} \in \Omega^{1}(Q), t \in[0,1]$ be a smooth family of 1 -forms so that $\omega_{t}:=\left.\omega_{W}\right|_{T Q}+d \alpha_{t}, t \in[0,1]$ is a smooth family of symplectic forms on $Q$ and so that $\left.\alpha_{t}\right|_{N}=0$ for all $t \in[0,1]$ and $\alpha_{0}=0$. Then there is a smooth family of symplectic embeddings $\iota_{t}: Q \hookrightarrow\left(W, \omega_{W}\right), t \in[0,1]$ which are arbitrarily $C^{0}$ close to $\iota_{0}$ so that $\iota_{1}^{*} \omega_{W}=\omega_{1}, \iota_{0}=\mathrm{id}_{Q}$ and so that $\left.\iota_{0}\right|_{U}=\left.\iota_{t}\right|_{U}$ for all $t \in[0,1]$.

We will now give a hint at why the lemma above is true. The details are contained in [EM02, Lemma 12.1.2]. First of all, we can $C^{1}$ approximate the family of 1-forms $\alpha_{t}$, $t \in[0,1]$ by a new family of 1 -forms $\alpha_{t}^{\prime}, t \in[0,1]$ so that

- $\alpha_{0}^{\prime}=\alpha_{0}, \alpha_{1}^{\prime}=\alpha_{1}$,

- for each compact codimension 0 submanifold $D \subset Q$, the function

$$
[0,1] \longrightarrow \Omega^{1}(D),\left.\quad t \rightarrow \alpha_{t}^{\prime}\right|_{D}
$$

is piecewise linear and

- for each $t \in[0,1]$ where $\left.\frac{d}{d t} \alpha_{t}^{\prime}\right|_{D}$ is well defined, there is a relatively compact open neighborhood $N_{D}$ of $D$ so that $\left.\frac{d}{d t} \alpha_{t}^{\prime}\right|_{N_{D}}$ is equal to $r d s$ where $r, s$ are smooth functions on $Q$ with compact support in $N_{D}-\bar{U}$.

See [EM02, 12.1.3]) for more details. After a bit more work, it is then sufficient to prove Lemma 6.28 when $\alpha_{t}=t r d s$ for some smooth compactly supported functions $r, s$ on $Q$. In this special case, the embeddings $\iota_{t}, t \in[0,1]$ are obtained by using the following Lemma:

Lemma 6.29. [EM02, 12.1.5] (Symplectic Twisting Lemma). Let $\left(Q, \omega_{Q}\right)$ be a symplectic manifold and let $\mathbb{D}^{2}(\epsilon) \subset \mathbb{C}$ be the open disk of radius $\epsilon$ with the standard symplectic form $d x \wedge d y$. Then for any compactly supported smooth functions $r, s$ on $Q$, there is a smooth function $\phi: Q \longrightarrow \mathbb{D}^{2}(\epsilon)$ so that the function

$$
\Phi: Q \longrightarrow Q \times \mathbb{D}^{2}(\epsilon), \quad \Phi(q):=(q, \phi(q))
$$

satisfies $\Phi^{*}\left(\omega_{Q}+d x \wedge d y\right)=\omega_{Q}+d r \wedge d s$.

Proof. In order to prove this lemma, one just needs to find $\phi$ so that $\phi^{*} d x \wedge d y=d r \wedge d s$. If $\epsilon>0$ was really large, then we could just choose $\phi=(r, s)$. However, $\epsilon$ could be really small and such a map would not be well defined. But this can be corrected by first finding a smooth area preserving immersion $\tau: \mathbb{D}^{2}(R) \rightarrow \mathbb{D}^{2}(\epsilon)$ for $R$ large and then letting $\phi=\tau \circ(r, s)$. Such an immersion is illustrated in Figure 21 below. Here we should think of the disk $\mathbb{D}^{2}(R)$ as being 'curled up' inside the disk $\mathbb{D}^{2}(\epsilon)$. Then $\phi=\tau \circ(r, s)$ for $R$ large has the properties we want. 


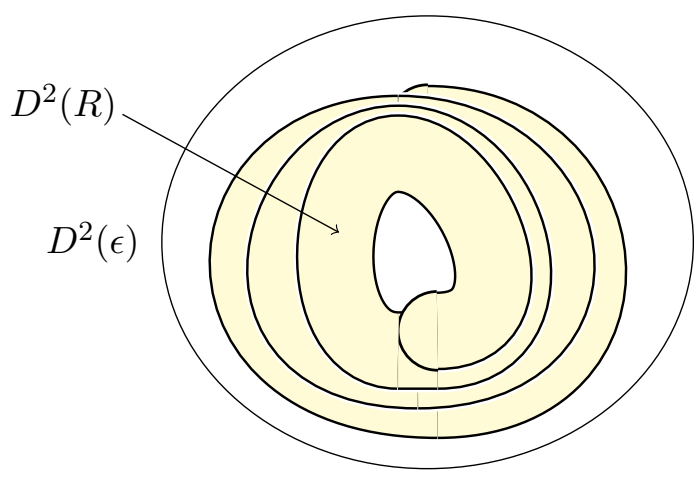

FIGURE 21. Immersion $\tau$.

Definition 6.30. Let $Q$ be a manifold and let $U \subset N \subset Q$ be open subsets. A smooth embedded homotopy to $N$ rel $U$ is a smooth family of embeddings $\iota_{t}: Q \hookrightarrow Q, t \in[0,1]$ so that

(1) $\iota_{0}=\mathrm{id}$,

(2) $\iota_{1}$ is a diffeomorphism onto $N$ and

(3) $\iota_{t}(u)=u$ for all $u \in U$ and $t \in[0,1]$.

Lemma 6.31. Let $\left(W, \omega_{W}\right),\left(\check{W}, \omega_{\breve{W}}\right)$ be symplectic manifolds and let $Q \subset W$ be a symplectic submanifold of positive codimension. Let $U, N \subset W$ be open sets so that $\bar{U} \subset N$ and define $U^{\prime}:=U \cap Q$ and $N^{\prime}:=N \cap Q$. Suppose also that $\overline{U^{\prime}}$ is a codimension 0 submanifold of $Q$ with the property that $H^{2}(Q ; \mathbb{R}) \longrightarrow H^{2}\left(U^{\prime} ; \mathbb{R}\right)$ is an injection and suppose $Q$ admits a smooth embedded homotopy to $N^{\prime}$ rel $U^{\prime}$. Let $\iota_{N}: N \hookrightarrow \breve{W}$ be a codimension 0 symplectic embedding. Then there is a neighborhood $V \subset W$ of $Q-\overline{U^{\prime}}$ and a codimension 0 symplectic embedding $\iota: U \cup V \hookrightarrow \check{W}$ so that $\left.\iota\right|_{U}=\left.\iota_{N}\right|_{U}$.

Proof. Let $\iota_{t}: Q \longrightarrow Q, t \in[0,1]$ be our smooth embedded homotopy to $N^{\prime}$ rel $U^{\prime}$. Since $\overline{U^{\prime}} \subset N^{\prime}$ is a codimension 0 submanifold, we can modify $\left(\iota_{t}\right)_{t \in[0,1]}$ so that it is a smooth embedded homotopy to $N^{\prime}$ rel $Y$ where $Y \subset N^{\prime}$ is a neighborhood of $\overline{U^{\prime}}$. After shrinking $Y$, we can also ensure that $\bar{Y} \subset N^{\prime}$ is a codimension 0 submanifold and that the inclusion map $U^{\prime} \hookrightarrow Y$ is a homotopy equivalence. Let $\omega_{t}:=\iota_{1-t}^{*} \omega_{W}$ for each $t \in[0,1]$. Since $\bar{Y}$ is a submanifold of $Q$ and since $H^{2}(Q ; \mathbb{R}) \longrightarrow H^{2}(\bar{Y} ; \mathbb{R})$ is an injection, there is a smooth family of 1-forms $\left(\alpha_{t}\right)_{t \in[0,1]}$ on $Q$ satisfying

- $\omega_{t}=\omega_{0}+d \alpha_{t}$ and

- $\left.\alpha_{t}\right|_{Y}=0$ for all $t \in[0,1]$ and $\alpha_{0}=0$.

Choose an open subset $\check{U} \subset Q$ so that $\overline{U^{\prime}} \subset \check{U}$ and $\check{\breve{U}} \subset Y$. Then by Lemma 6.28, there is a smooth family of symplectic embeddings

$$
\nu_{t}: Q-\overline{U^{\prime}} \hookrightarrow\left(\check{W}-\overline{\iota_{N}(U)}, \omega_{\breve{W}}\right), \quad t \in[0,1]
$$

so that $\nu_{0}(y)=\iota_{N}\left(\iota_{1}(y)\right)$ for each $y \in Q, \nu_{t}(y)=\iota_{N}(y)$ for all $y \in \check{U}-\overline{U^{\prime}}$ and $\nu_{1}^{*} \omega_{\breve{W}}=\omega_{1}$. Hence by Lemma 6.27, there is a neighborhood $V \subset W$ of $Q-\overline{U^{\prime}}$ and a codimension 0 symplectic embedding $\iota: U \cup V \longrightarrow \check{W}$ so that $\left.\iota\right|_{U}=\left.\iota_{N}\right|_{U}$ and $\left.\iota\right|_{Q}=\nu_{1}$.

Lemma 6.32. Every partly stratified symplectic subset $S \subset M$ admits a weak curled up embedding. 
Proof. Let $S_{1}, \cdots, S_{l}$ be the strata of $S$. Suppose (by induction) that $S_{<j}:=\cup_{i<j} S_{i}$ admits a weakly curled up embedding $\check{\iota}: \check{N} \hookrightarrow \check{M}_{<1}$ for some $j \in\{1, \cdots, l\}$. Since $\check{\iota}(y)=y$ for each $y$ in a neighborhood of $\check{N} \cap(M \times \mathbb{T}) \subset \check{M}$, there is a neighborhood $\check{U} \subset \check{M}_{<1}$ of $M \times \mathbb{T}$ with the property that

$$
\widetilde{\iota}: \check{U} \cup \check{N} \longrightarrow \check{M}, \quad \widetilde{\iota}(y):= \begin{cases}\check{\iota}(y) & \text { if } y \in \check{N} \\ y & \text { if } y \in \check{U}\end{cases}
$$

is a smooth map after shrinking $\check{N}$ by an arbitrarily small amount. Let $f_{\check{U} \cup \check{N}}: M \times \mathbb{T} \longrightarrow$ $\mathbb{R} \cup\{\infty\}$ be the lower semi-continuous function given in Equation (6.18) with $N$ replaced by $\check{U} \cup \check{N}$ (see Lemma 6.25). Now $f_{\check{U} \cup \check{N}}=\infty$ along $S_{<j}$. Combining this with the fact that $f_{\breve{U} \cup \check{N}}$ is positive and lower semi-continuous and the fact that $S_{<j} \times \mathbb{T}$ is compact, we can find a smooth function $\tilde{f}:\left(M-S_{<j}\right) \times \mathbb{T} \longrightarrow \mathbb{R}_{>0}$ satisfying $\widetilde{f}<f_{\check{U} \cup \check{N}}$ so that for any sequence of points $\left(x_{j}\right)_{j \in \mathbb{N}}$ in $\left(M-S_{<j}\right) \times \mathbb{T}$ converging to a point in $S_{<j} \times \mathbb{T}$, we have $\tilde{f}\left(x_{j}\right) \rightarrow \infty$ as $j \rightarrow \infty$. In particular,

$$
U:=\left(S_{<j} \times T^{*} \mathbb{T}\right) \cup \check{M}_{<\tilde{f}} \mid\left(M-S_{<j}\right) \times \mathbb{T}
$$

is an open neighborhood of $(M \cup \mathbb{T}) \cup\left(S_{<j} \times T^{*} \mathbb{T}\right)$. See Figure 22 .

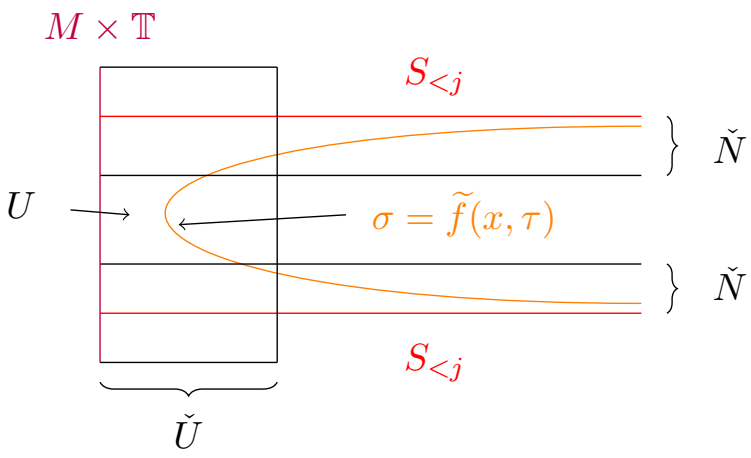

Figure 22. Picture of $\left.\check{M}_{<f}\right|_{S_{j} \times \mathbb{T}}$.

Define $Q:=S_{j} \times T^{*} \mathbb{T}$ and $N:=\check{M}_{<\check{U} \cup \check{N}}$. Define $U^{\prime}:=U \cap Q, N^{\prime}:=N \cap Q$. Then $\overline{U^{\prime}}$ is a codimension 0 submanifold of $Q$ with the property that $H^{2}(Q ; \mathbb{R}) \longrightarrow H^{2}\left(U^{\prime} ; \mathbb{R}\right)$ is injective. Also $\bar{U} \subset N$ and the manifold $Q$ admits a smooth embedded homotopy to $N^{\prime}$ rel $U^{\prime}$. Hence by Lemma 6.31 with $U, N, U^{\prime}, N^{\prime}$ and $Q$ as above and with $W=\check{M}, \check{W}=\check{M}_{<1}$ and $\iota_{N}=\left.\widetilde{\iota}\right|_{N}$, there is a neighborhood $V \subset \check{M}$ of $Q-\overline{U^{\prime}}$ and a codimension 0 symplectic embedding $\iota: U \cup V \longrightarrow \check{M}_{<1}$ so that $\left.\iota\right|_{U}=\widetilde{\iota}_{U}$. Since $\iota(y)=y$ for all $y$ sufficiently near $M \times \mathbb{T}$, we get that $\iota$ is a weakly curled up embedding of $S_{<j+1}=S_{<j} \cup S_{j}$. Hence we are done by induction on $j \in\{1, \cdots, l\}$.

Proof of Proposition 6.20. Let $S$ be our partly stratified symplectic subset. By Lemma 6.32 $S \times T^{*} \mathbb{T}$ admits a weak curled up embedding. Hence by Lemma $6.26, S \times T^{*} \mathbb{T}$ admits a curled up embedding. Therefore by Lemma $6.23, S$ is stably displaceable. 


\section{Proof of the Main Theorem}

By Calabi-Yau manifold, we will just mean a smooth complex projective variety with trivial first Chern class. We have the following lemma:

Lemma 7.1. ([Bat99, Proposition 3.1]). Let $\widehat{\Phi}: X \rightarrow \widehat{X}$ be a birational equivalence between smooth projective Calabi-Yau manifolds. Then there are complex codimension $\geq 2$ subvarieties $V_{X} \subset X$ and $V_{\widehat{X}} \subset \widehat{X}$ and an isomorphism

$$
\Phi: X-V_{X} \stackrel{\cong}{\longrightarrow}-V_{\widehat{X}}
$$

equal to $\left.\widehat{\Phi}\right|_{X-V_{X}}$.

Note that the Calabi-Yau condition here is crucial. For instance, if we take a smooth projective variety and blow it up at a point, then we create a birational variety whose second Betti number is strictly larger. Even though the proof of lemma 7.1 is contained in [Bat99, Proposition 3.1], we give a more detailed version of the same proof for readers who may not be experts in algebraic geometry. There is also a more general version of this lemma (see [KM98, Theorem 3.52]), which proves the same statement for varieties which are 'minimal' in some sense.

Proof of Lemma 7.1. Morally, the idea of the proof is as follows. If the region where $\widehat{\Phi}$ is not a submersion had complex codimension 1 then $\widehat{\Phi}$ would contract a codimension 1 subvariety $Z$ to a lower dimensional variety (See Definition 6.14). One can then show that such a contraction would ensure that $c_{1}(X)$ or $c_{1}(\widehat{X})$ is non-trivial giving us a contradiction. A similar argument also applies to the inverse $\widehat{\Phi}^{-1}$ and this gives us our result. Therefore, our proof comes in two parts:

Part (1) Showing that if the region where $\widehat{\Phi}$ is not a submersion has codimension less than 2, then $\widehat{\Phi}$ contracts a divisor.

Part (2) Showing that $c_{1}(X)$ or $c_{1}(\widehat{X})$ is non-trivial due to the existence of such a divisor.

Part (1): Since $\widehat{X}$ is a projective variety, we have that $\widehat{X}$ is a closed subvariety of $\mathbb{C} P^{N}$ for some $N \in \mathbb{N}$. Let $\iota: \widehat{X} \hookrightarrow \mathbb{C} P^{N}$ be the inclusion map. Consider the rational map $f=\iota \circ \widehat{\Phi}$. In any holomorphic chart $z_{1}, \cdots, z_{n}$ on $X$, the map $f$ has the form $\left[\phi_{0}\left(z_{1}, \cdots, z_{n}\right), \cdots, \phi_{N}\left(z_{1}, \cdots, z_{n}\right)\right]$ in homogeneous coordinates on $\mathbb{C} P^{N}$ where $\phi_{0}, \cdots, \phi_{N}$ are holomorphic functions. This local description of $f$ does not change if we multiply $\phi_{0}, \cdots, \phi_{N}$ by a common holomorphic function. In particular, if $\phi_{0}, \cdots, \phi_{N}$ all vanish to the same order along some codimension 1 subvariety, then we can remove a common factor from all of these holomorphic functions so that $\phi_{0}^{-1}(0) \cap \cdots \cap \phi_{N}^{-1}(0)$ has codimension 2 or higher after shrinking the chart ([GH94, Chapter 0, Section 1]). As a result, we get that there is a codimension $\geq 2$ subvariety $V_{X} \subset X$ so that $\widehat{\Phi}$ is well defined outside $V_{X}$. Now suppose that $\widehat{\Phi}: X-V_{X} \longrightarrow \widehat{X}$ is not an isomorphism onto its image. Then by [Sha13, Theorem 2.16], there is a codimension 1 subvariety $Z \subset X-V_{X}$ which is contracted by $\widehat{\Phi}$ as in Definition 6.14.

Part (2): Let $V_{X}$ and $Z$ be as above. We now wish to show that the existence of the variety $Z$ implies that either $c_{1}(X)$ or $c_{1}(\widehat{X})$ is non-trivial, and therefore giving us a contradiction. Consider the relative canonical bundle $\kappa_{X / \widehat{X}}:=\kappa_{X} \otimes \widehat{\Phi}^{*} \kappa_{\widehat{X}}$ on $X-V_{X}$ where $\kappa_{X}$ and $\kappa_{\widehat{X}}$ are the canonical bundles of $X$ and $\widehat{X}$ respectively (Definition 6.14). Then $\kappa_{X / \widehat{X}}$ can be 
identified with the bundle $\operatorname{Hom}\left(\kappa_{X}^{*}, \widehat{\Phi}^{*} \kappa_{\widehat{X}}^{*}\right)$ where $\kappa_{X}^{*}$ and $\kappa_{\widehat{X}}^{*}$ are the anti-canonical bundles of $X$ and $\widehat{X}$ respectively (See Definition 6.13). Under this identification, we have a section $s$ of $\kappa_{X / \widehat{X}}$ given by the Jacobian of $\widehat{\Phi}$, which is the map sending $v_{1} \wedge \cdots \wedge v_{1} \in \kappa_{X}^{*}$ to $D \pi\left(v_{1}\right) \wedge \cdots \wedge D \pi\left(v_{n}\right) \in \widehat{\Phi}^{*} \kappa_{\widehat{X}}^{*}$. This holomorphic section $s$ vanishes along $Z$. Now since $V_{X}$ has complex codimension $\geq 2$, we have by using Harthog's theorem and the Cauchy integral formula [GH94, Chapter 0, Section 1], that the bundle $\kappa_{X / \widehat{X}}$ and the section $s$ extends to a holomorphic bundle $L$ over $X$ together with a holomorphic section $\check{s}$ of $L$. We have that $c_{1}(L)$ is Poincaré dual to $[(\check{s})]$ by ([GH94, Chapter 1 , Section 1]), where $(\check{s})$ and the homology class $[(\check{s})]$ are given in Definition 6.14. Choose a Kähler form $\omega$ on $X$. Then $\omega^{n-1}$ is a positive volume form on the smooth locus of any codimension 1 subvariety. Therefore the de-Rham cohomology class $\left[\omega^{n-1}\right]$ on $X$ pairs non-trivially with the homology class $[(\check{s})]$ since $(\check{s})$ is a non-trivial effective divisor (See Definition 6.14). Hence $c_{1}(L)$ is non-zero. Since $V_{X}$ is a complex codimension $\geq 2$ subvariety, we get that the natural restriction map $H^{2}(X ; \mathbb{Z}) \longrightarrow H^{2}\left(X-V_{X} ; \mathbb{Z}\right)$ is an isomorphism. This implies that $c_{1}\left(\kappa_{X / \widehat{X}}\right) \neq 0$ in $X-V_{X}$. Since $c_{1}\left(\kappa_{X / \widehat{X}}\right)=c_{1}\left(\left.\kappa_{X}\right|_{X-V_{X}}\right)-\widehat{\Phi}^{*} c_{1}\left(\kappa_{\widehat{X}}\right)$, either $c_{1}(X)$ or $c_{1}(\widehat{X})$ is non-zero giving us a contradiction. Hence $\widehat{\Phi}$ maps $X-V_{X}$ isomorphically to its image.

Similar reasoning can be used to show that there is a codimension $\geq 2$ subvariety $\check{V}_{\widehat{X}} \subset \widehat{X}$ so that $\widehat{\Phi}^{-1}$ maps $\widehat{X}-\check{V}_{\widehat{X}}$ isomorphically to an open subset of $X$ containing $X-V_{X}$. Hence $V_{\widehat{X}}:=\widehat{X}-\widehat{\Phi}\left(X-V_{X}\right)$ has codimension $\geq 2$ and our lemma holds.

We have the following immediate corollary of Lemma 7.1.

Corollary 7.2. Let $X, \widehat{X}, \widehat{\Phi}, V_{X}, V_{\widehat{X}}$ and $\Phi$ be as in Lemma \%.1. Let $D \subset X-V_{X}$ be a compact submanifold possibly with boundary and let $R$ be any ring. Then there is a natural identification of homology and cohomology groups

$$
\begin{aligned}
& \Phi_{*}: H_{2}(X-D ; R) \stackrel{\cong}{\longleftarrow} H_{2}\left(X-V_{X}-D ; R\right) \underset{\Phi_{*}}{\cong} H_{2}\left(\widehat{X}-V_{\widehat{X}}-\Phi(D) ; R\right) \stackrel{\cong}{\longrightarrow} H_{2}(\widehat{X}-\Phi(D) ; R) \text {. } \\
& \Phi^{*}: H^{2}(\widehat{X}, \Phi(D) ; R) \stackrel{\cong}{\longrightarrow} H^{2}\left(\widehat{X}-V_{\widehat{X}}, \Phi(D) ; R\right) \underset{\Phi^{*}}{\cong} H^{2}\left(X-V_{X}, D ; R\right) \stackrel{\cong}{\longleftarrow} H^{2}(X, D ; R) .
\end{aligned}
$$

Remark 7.3. From now on we will fix the notation $\widehat{\Phi}, \Phi, X, \widehat{X}, V_{X}$ and $V_{\widehat{X}}$. Also we will not distinguish between $H_{2}(X-D ; R)$ and $H_{2}(\widehat{X}-\Phi(D) ; R)$ (resp. $H^{2}(X, D ; R)$, $\left.H^{2}(\widehat{X}, \Phi(D) ; R)\right)$ for each compact submanifold with boundary $D \subset X-V_{X}$ and each ring $R$.

The following lemma finds for us an appropriate common Zariski dense affine variety on $X$ and $\widehat{X}$. This lemma will also be used to construct appropriate Kähler forms on $X$ and $\widehat{X}$ which agree on a large compact subset of this common affine subvariety. We will use the notation from Definition 6.14.

Lemma 7.4. Let $\omega_{X}$ and $\omega_{\widehat{X}}$ be Kähler forms on $X$ and $\widehat{X}$ and suppose that their corresponding De Rham cohomology classes admit lifts to integral cohomology classes $\left[\omega_{X}\right] \in H^{2}(X ; \mathbb{Z})$ and $\left[\omega_{\widehat{X}}\right] \in H^{2}(\widehat{X} ; \mathbb{Z})$ respectively. Then there are effective divisors $\Delta$ on $X$ and $\widehat{\Delta}$ on $\widehat{X}$ and an integer $\mu>0$ so that

(1) $[\Delta]^{*}=\mu\left[\omega_{X}\right]$ and $[\widehat{\Delta}]^{*}=\mu\left[\omega_{\widehat{X}}\right]$, 
(2) $V_{X} \subset \operatorname{supp}(\Delta), V_{\widehat{X}} \subset \operatorname{supp}(\widehat{\Delta})$,

(3) $\overline{\Phi\left(\operatorname{supp}(\Delta)-V_{X}\right)}=\operatorname{supp}(\widehat{\Delta})$ and

(4) $A:=X-\operatorname{supp}(\Delta)$ and $\widehat{A}:=\widehat{X}-\operatorname{supp}(\widehat{\Delta})$ are affine varieties.

Proof. By [GH94, Chapter 1, Section 2], we can find a collection $\Delta_{1}, \cdots, \Delta_{l}$ of irreducible codimension 1 subvarieties of $X$ so that $\left[\Delta_{1}\right]^{*}, \cdots,\left[\Delta_{l}\right]^{*}$ generates $H^{1,1}(X) \cap H^{2}(X ; \mathbb{Z})$ and so that $\left[\widehat{\Delta}_{1}\right]^{*}, \cdots,\left[\widehat{\Delta}_{l}\right]^{*}$ generates $H^{1,1}(\widehat{X}) \cap H^{2}(\widehat{X} ; \mathbb{Z})$ where $\widehat{\Delta_{i}}=\overline{\Phi\left(\Delta_{i}-V_{X}\right)}$ for each $i=1, \cdots, l$. We can enlarge this collection of varieties so that (2) is satisfied. Choose a positive integer $N>0$ large enough so that

$$
\left[\omega_{X}\right]-\frac{1}{N} \sum_{i=1}^{l}\left[\Delta_{i}\right]^{*}, \quad\left[\omega_{\widehat{X}}\right]-\frac{1}{N} \sum_{i=1}^{l}\left[\widehat{\Delta}_{i}\right]^{*}
$$

represent Kähler forms. Such an $N$ exists since being Kähler is an open condition among $(1,1)$-forms with respect to the $C^{0}$-topology. By the Kodaira embedding theorem [GH94, Chapter 1, Section 4], we can find a positive integer $m$ and codimension 1 subvarieties $\Upsilon$ on $X$ and $\widehat{\Upsilon}^{\prime}$ on $\widehat{X}$ so that

$$
[\Upsilon]^{*}=m N\left[\omega_{X}\right]-m \sum_{i=1}^{l}\left[\Delta_{i}\right]^{*}, \quad\left[\widehat{\Upsilon}^{\prime}\right]^{*}=m N\left[\omega_{\widehat{X}}\right]-m \sum_{i=1}^{l}\left[\widehat{\Delta}_{i}\right]^{*} .
$$

Let $\widehat{\Upsilon}:=\overline{\Phi\left(\Upsilon-V_{X}\right)}$ and $\Upsilon^{\prime}=\overline{\Phi^{-1}\left(\widehat{\Upsilon}^{\prime}-V_{\widehat{X}}\right)}$. Since $\left[\Delta_{1}\right]^{*}, \cdots,\left[\Delta_{l}\right]^{*}$ generates $H^{1,1}(X) \cap$ $H^{2}(X ; \mathbb{Z})$, and $\left[\widehat{\Delta}_{1}\right]^{*}, \cdots,\left[\widehat{\Delta}_{l}\right]^{*}$ generates $H^{1,1}(\widehat{X}) \cap H^{2}(\widehat{X} ; \mathbb{Z})$, we have by [GH94, Chapter 1 , Section 2] that there are integers $\left(a_{i}\right)_{i=1}^{l},\left(\widehat{a}_{i}\right)_{i=1}^{l}$ so that

$$
\sum_{j=1}^{l} a_{i}\left[\Delta_{i}\right]=\left[\Upsilon^{\prime}\right], \quad \sum_{j=1}^{l} \widehat{a}_{i}\left[\widehat{\Delta}_{i}\right]=[\widehat{\Upsilon}]
$$

Now choose a positive integer $m^{\prime}$ greater than $\max _{i=1}^{l} a_{i}$ and $\max _{i=1}^{l} \widehat{a}_{i}$. Define

$$
\Delta:=m^{\prime} \Upsilon+\Upsilon^{\prime}+\sum_{i=1}^{l}\left(m^{\prime} m-a_{i}\right) \Delta_{i}, \quad \widehat{\Delta}:=\widehat{\Upsilon}+m^{\prime} \widehat{\Upsilon}^{\prime}+\sum_{i=1}^{l}\left(m^{\prime} m-\widehat{a}_{i}\right) \widehat{\Delta}_{i} .
$$

Then by Equations (7.3) and (7.4), we have that $[\Delta]^{*}=m^{\prime} m N\left[\omega_{X}\right]$ and $[\widehat{\Delta}]^{*}=m^{\prime} m N\left[\omega_{\widehat{X}}\right]$ and hence (1) holds with $\mu=m^{\prime} m N$. Also $\Delta$ and $\widehat{\Delta}$ are effective divisors with support

$$
\operatorname{supp}(\Delta)=\Upsilon \cup \Upsilon^{\prime} \cup \bigcup_{i=1}^{l} \Delta_{i}, \quad \operatorname{supp}(\widehat{\Delta})=\widehat{\Upsilon} \cup \widehat{\Upsilon}^{\prime} \cup \bigcup_{i=1}^{l} \widehat{\Delta}_{i}
$$

and hence (2) and (3) are satisfied. Finally $A$ and $\widehat{A}$ are affine varieties by the Kodaira embedding theorem [GH94, Chapter 1, Section 4].

We will now prove our main result (Theorem 1.2). Here is a statement of this theorem: Let $\omega_{X}$ and $\omega_{\widehat{X}}$ be Kähler forms on $X$ and $\widehat{X}$ respectively whose cohomology classes lift to integer cohomology classes. Then there exists a graded $\Lambda_{\mathbb{K}}^{\omega_{X}, \omega_{\widehat{X}}}$-algebra $Z$ and algebra isomorphisms

$$
Z \otimes_{\Lambda_{\mathbb{K}}^{\omega_{X}, \omega_{\widehat{X}}}} \Lambda_{\mathbb{K}}^{\omega_{X}} \stackrel{\cong}{\longrightarrow} Q H^{*}\left(X ; \Lambda_{\mathbb{K}}^{\omega_{X}}\right), \quad Z \otimes_{\Lambda_{\mathbb{K}}^{\omega_{X}, \omega_{\widehat{X}}}} \Lambda_{\mathbb{K}}^{\omega_{\widehat{X}}} \stackrel{\cong}{\longrightarrow} Q H^{*}\left(X ; \Lambda_{\mathbb{K}}^{\omega_{\widehat{X}}}\right)
$$


over the Novikov rings $\Lambda_{\mathbb{K}}^{\omega_{X}}$ and $\Lambda_{\mathbb{K}}^{\omega_{\widehat{X}}}$ respectively where $\Lambda_{\mathbb{K}}^{\omega_{X}, \omega_{\widehat{X}}}, \Lambda_{\mathbb{K}}^{\omega_{X}}$ and $\Lambda_{\mathbb{K}}^{\omega_{\widehat{X}}}$ are the Novikov rings given in Example 2.5\%.

Proof of Theorem 1.2. Let $n$ be the complex dimension of $X$. Let $\Delta, \widehat{\Delta}, \mu, A$ and $\widehat{A}$ be as in Lemma 7.4. By the divisor line bundle correspondence [GH94, Chapter 1, Section 1], there are holomorphic line bundles $L \longrightarrow X, \widehat{L} \longrightarrow \widehat{X}$ with holomorphic sections $s$ and $\widehat{s}$ respectively so that we have an equality of divisors $\left(s^{-1}(0)\right)=\Delta$ and $\left(\widehat{s}^{-1}(0)\right)=\widehat{\Delta}$ respectively. Choose Hermitian metrics $|\cdot|$ and $|\cdot|^{\prime}$ on $L$ and $\widehat{L}$ respectively so that $-d d^{c} \rho=\left.\mu \omega_{X}\right|_{A}$ and $-d d^{c} \widehat{\rho}=$ $\left.\mu \omega_{\widehat{X}}\right|_{\widehat{A}}$ where $\rho:=-\log (|s|)$ and $\widehat{\rho}:=-\log \left(|\widehat{s}|^{\prime}\right)$.

By Corollary 6.21 , there exists a constant $C>0$ so that $X-K$ is stably displaceable inside the symplectic manifold $\left(X, \mu \omega_{X}\right)$ where $K:=\rho^{-1}((-\infty, C])$. Also by Lemma 6.3 we can enlarge $C$ so that the interior of $K$ contains the skeleton of $\rho$ (Definition 6.4). Therefore by a Moser argument ([MS98, Exercise 3.36]), there is a contact cylinder $\check{C}_{4} \subset A$ inside the symplectic manifold $\left(X, \mu \omega_{X}\right)$ whose associated Liouville domain is $D_{4}:=K$. Define $\widehat{C}_{4}:=\Phi\left(\check{C}_{4}\right)$ and $\widetilde{D}_{4}:=\Phi\left(D_{4}\right)$.

By Proposition 6.7, there is an index bounded contact cylinder $\check{C}_{3}=\left[1-\epsilon_{3}, 1+\epsilon_{3}\right] \times C_{3} \subset A$ inside the symplectic manifold $\left(X, \mu \omega_{X}\right)$ so that the interior of the associated Liouville domain $D_{3}$ contains $D_{4}$ and is contained in $A$. Define $\widehat{C}_{3}:=\Phi\left(\check{C}_{3}\right)$ and $\widetilde{D}_{3}:=\Phi\left(D_{3}\right)$.

By Corollary 6.5, there exists a partially algebraic plurisubharmonic function $\check{\rho}: \widehat{A} \longrightarrow \mathbb{R}$, a compact set $K^{\prime}$ of $A$ containing $D_{3} \cup \check{C}_{3}$ so that

(1) $\left.\Phi^{*}(\check{\rho})\right|_{D_{3} \cup \check{C}_{3}}=\left.\rho\right|_{D_{3} \cup \check{C}_{3}}$

(2) $\check{\rho}=\kappa_{1}\left(\widehat{\rho}-\log \left(\kappa_{2}\right)\right)$ outside $\Phi\left(K^{\prime}\right)$ for some large $\kappa_{1}, \kappa_{2} \in \mathbb{N}$ and

(3) the skeleton of $\Phi^{*}(\check{\rho})$ is equal to the skeleton of $\rho$.

Define $\omega_{X}^{\prime}:=\mu \omega_{X}$ and

$$
\omega_{\widehat{X}}^{\prime}:=\left\{\begin{array}{ll}
\kappa_{1} \mu \omega_{\widehat{X}} & \text { inside } X-\Phi\left(K^{\prime}\right) \\
-d d^{c} \check{\rho} & \text { otherwise. }
\end{array}\right\}
$$

By Corollary 6.21, there is a compact subset $\widehat{K} \subset \widehat{X}$ whose interior contains $\Phi\left(K^{\prime}\right)$ so that $X-\widehat{K}$ is stably displaceable in $\left(\widehat{X}, \omega_{\widehat{X}}^{\prime}\right)$. By Proposition 6.7 , there exists an index bounded contact cylinder $\widehat{C}=[1-\epsilon, 1+\epsilon] \times C$ in the symplectic manifold $\left(\widehat{X}, \omega_{\widehat{X}}^{\prime}\right)$ which is disjoint from $\cup_{j \in S} \widehat{\Delta}_{j}$ and $\widehat{K}$ and whose associated Liouville domain $\widehat{D}$ is contained in $\widehat{A}$ and whose interior contains $\widehat{K}$. By the same proposition, we can also assume that $\widehat{C}$ contains a contact cylinder $\widehat{C}_{1}$ whose associated Liouville domain $\widetilde{D}_{1}$ contains $\widehat{K}$ and so that a Liouville form associated to $\widehat{C}_{1}$ is the restriction of $-d^{c} \check{\rho}$ to $\widetilde{D}$. By a Moser argument ([MS98, Exercise 3.36]) we can construct contact cylinders $\widehat{C}_{0}:=\left[1-\epsilon_{0}, 1+\epsilon_{0}\right] \times C_{0}$ and $\widehat{C}_{2}$ inside $\left(\widehat{X}, \omega_{\widehat{X}}^{\prime}\right)$ whose associated Liouville domains are $\widehat{D}_{0}=\widehat{D} \cup \widehat{C}$ and $\widetilde{D}_{2}=\widehat{\widehat{D}-\widehat{C}}$. I.e. they have boundary equal to $\{1+\epsilon\} \times C$ and $\{1-\epsilon\} \times C$ respectively. Also we can assume $\widetilde{D}_{3} \subset \widetilde{D}_{2} \subset \widetilde{D}_{1} \subset \widetilde{D}_{0}$. We define $\check{C}_{i}:=\Phi^{-1}\left(\widehat{C}_{i}\right), D_{i}:=\Phi^{-1}\left(\widetilde{D}_{i}\right)$ for $i=0,1,2$ (See Figure 23 ).

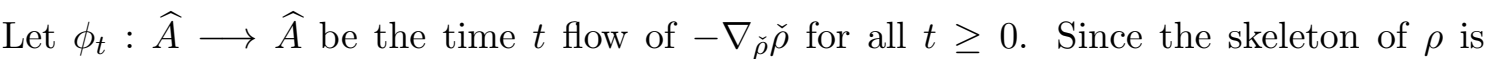
contained in the interior of $D_{4}$, we get that the skeleton of $\check{\rho}$ is contained in the interior of $\widetilde{D}_{4}$ by (3). Therefore we have that there is a constant $T>0$ so that $\phi_{T}\left(\widetilde{D}_{3}\right) \subset \widetilde{D}_{4}$ for some $T>0$. Since the inclusions $\widetilde{D}_{4} \subset \widetilde{D}_{1}, \phi_{T}\left(\widetilde{D}_{3}\right) \subset \widetilde{D}_{3}$ and $\widetilde{D}_{2} \subset \widetilde{D}_{0}$ induce isomorphisms

$$
H^{2}\left(\widehat{X}, \widetilde{D}_{1} ; \mathbb{R}\right) \cong H^{2}\left(\widehat{X}, \widetilde{D}_{4} ; \mathbb{R}\right), \quad H^{2}\left(\widehat{X}, \widetilde{D}_{3} ; \mathbb{R}\right) \cong H^{2}\left(\widehat{X}, \phi_{T}\left(\widetilde{D}_{3}\right) ; \mathbb{R}\right),
$$


$X$
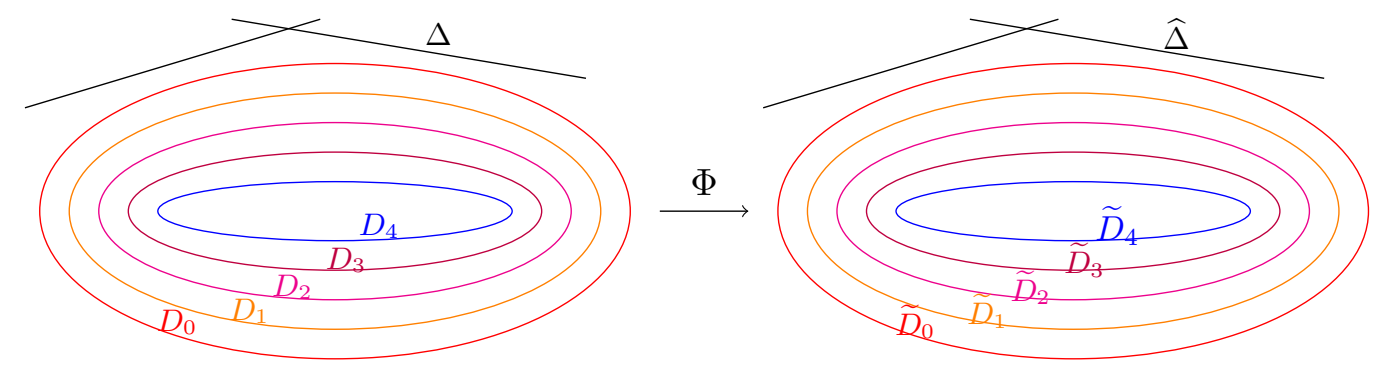

$\widehat{X}$

FiguRE 23. Liouville domains in $X$ and $\widehat{X}$.

$$
H^{2}\left(\widehat{X}, \widetilde{D}_{0} ; \mathbb{R}\right) \cong H^{2}\left(\widehat{X}, \widetilde{D}_{2} ; \mathbb{R}\right)
$$

we get that the restriction map $H^{2}\left(\widehat{X}, \widetilde{D}_{i} ; \mathbb{R}\right) \longrightarrow H^{2}\left(\widehat{X}, \widetilde{D}_{j} ; \mathbb{R}\right)$ is an isomorphism for each $0 \leq i \leq j \leq 4$. Also we have natural isomorphisms $H^{2}\left(X, D_{i} ; \mathbb{R}\right) \longrightarrow H^{2}\left(\widehat{X}, \widetilde{D}_{i} ; \mathbb{R}\right)$ for each $i=0,1,2,3,4$ by Corollary 7.2. So from now on we will identify all of these cohomology groups.

Since the quantum cohomology groups and the associated Novikov rings in the statement of our theorem only depend on $\omega_{X}$ and $\omega_{\widehat{X}}$ up to scalar multiplication and up to adding an exact 2 -form, we can just replace $\omega_{X}$ and $\omega_{\widehat{X}}$ with $\omega_{X}^{\prime}$ and $\omega_{\widehat{X}}^{\prime}$. Hence from now on, we'll assume $\omega_{X}=\omega_{X}^{\prime}$ and $\omega_{\widehat{X}}=\omega_{\widehat{X}}^{\prime}$.

Let $\widetilde{\omega} \in \Omega^{2}(X)$ be a $\check{C}_{2}$-compatible 2 -form which is equal to 0 inside $D_{3}$ and $\omega_{X}$ outside $D_{3} \cup\left(\left[1,1+\epsilon_{3} / 2\right] \times C_{3}\right)$. Let $\widetilde{\omega}^{\prime} \in \Omega^{2}(\widehat{X})$ be a $\check{C}_{0}$-compatible 2 -form which is equal to 0 inside $\widetilde{D}_{0}$ and $\omega_{\widehat{X}}$ outside $\widetilde{D}_{0} \cup\left(\left[1,1+\epsilon_{0} / 2\right] \times C_{0}\right)$. In the unlikely event that $[\widetilde{\omega}] \in H^{2}(X ; \mathbb{R})$ is proportional to $\left[\widetilde{\omega}^{\prime}\right] \in H^{2}(\widehat{X} ; \mathbb{R})$, we rescale $\widetilde{\omega}^{\prime}$ so that these cohomology classes are equal (this is done to ensure that the cones constructed below satisfy condition (1) of Definition 2.58). Let $Q_{+} \subset H^{2}\left(X, D_{3} ; \mathbb{R}\right) \times \mathbb{R} \times \mathbb{R}=H^{2}\left(\widehat{X}, \widetilde{D}_{0} ; \mathbb{R}\right) \times \mathbb{R} \times \mathbb{R}$ be the cone spanned by $([\widetilde{\omega}], 1,1)$ and $\left(\left[\widetilde{\omega}^{\prime}\right], 1,1\right)$. Let $Q_{-} \subset H^{2}\left(X, D_{3} ; \mathbb{R}\right) \times \mathbb{R} \times \mathbb{R}$ be the cone spanned by $([\widetilde{\omega}], 0,1),([\widetilde{\omega}], 1,1)\left(\left[\widetilde{\omega}^{\prime}\right], 0,1\right)$ and $\left(\left[\widetilde{\omega}^{\prime}\right], 1,1\right)$. Let $Q_{+}^{C_{3}} \subset H^{2}\left(X, D_{3} ; \mathbb{R}\right) \times \mathbb{R} \times \mathbb{R}$, (resp. $Q_{+}^{\widehat{C}_{0}} \subset H^{2}\left(X, D_{3} ; \mathbb{R}\right) \times \mathbb{R} \times \mathbb{R}$ ) be the one dimensional cone spanned by $([\widetilde{\omega}], 1,1$ ) (resp. $\left.\left(\left[\widetilde{\omega}^{\prime}\right], 1,1\right)\right)$. Let $Q_{-}^{\check{C}_{3}} \subset H^{2}\left(X, D_{3} ; \mathbb{R}\right) \times \mathbb{R} \times \mathbb{R},\left(\operatorname{resp} . Q_{-}^{\widehat{C}_{0}} \subset H^{2}\left(X, D_{3} ; \mathbb{R}\right) \times \mathbb{R} \times \mathbb{R}\right)$ be the two dimensional cone spanned by $([\widetilde{\omega}], 0,1)$ and $([\widetilde{\omega}], 1,1)\left(\operatorname{resp} .\left(\left[\widetilde{\omega}^{\prime}\right], 0,1\right),\left(\left[\widetilde{\omega}^{\prime}\right], 1,1\right)\right)$.

Since the interior of $D_{3}$ contains $D_{4}=K$, we have that $\overline{X-D_{3}}$ is stably displaceable inside $\left(X, \omega_{X}\right)$ and hence we have an isomorphism of $\Lambda_{\mathbb{K}}^{Q_{+}^{\check{C}_{3}}}=\Lambda_{\mathbb{K}}^{\omega_{X}}$-algebras

$$
S H_{\check{C}_{3}, Q_{-}^{\check{C}_{3}}, Q_{+}^{\check{C}_{3}}}^{*}\left(D_{3} \subset X\right) \cong Q H^{*}\left(X, \Lambda_{\mathbb{K}}^{\omega_{X}}\right),
$$

and

$$
\stackrel{\lim }{\longrightarrow} \lim ^{1} \mathbb{S} H_{\check{C}_{3}, Q_{-}^{\check{C}_{3}}, Q_{+}^{\check{C}_{3}}}^{*}\left(D_{3} \subset X\right)=0 .
$$

by Theorems 5.10, 5.12 and Propositions 5.4 and 5.24. Similarly, we have an isomorphism of $\Lambda_{\mathbb{K}}^{Q_{+}^{\widehat{C}_{0}}}=\Lambda_{\mathbb{K}}^{\omega_{\widehat{X}}}$-algebras

$$
S H_{\widehat{C}_{0}, Q_{-}^{\widehat{C}_{0}}, Q_{+}^{\widehat{C}_{0}}}^{*}\left(\widetilde{D}_{0} \subset \widehat{X}\right) \cong Q H^{*}\left(X, \Lambda_{\mathbb{K}}^{\omega_{\widehat{X}}}\right),
$$


and

$$
\stackrel{\lim }{\longrightarrow} \lim ^{1} \mathbb{S} H_{\widehat{C}_{0}, Q_{-}^{\widehat{C}_{0}}, Q_{+}^{\widehat{C}_{0}}}^{*}\left(\widetilde{D}_{0} \subset \widehat{X}\right)=0 .
$$

By Proposition 5.32 applied to $\left(\widehat{C}_{i}\right)_{i=0,1,2,3,4}$ and $\left(\widetilde{D}_{i}\right)_{i=0,1,2,3,4}$ we have that the transfer map

$$
\mathbb{S} H_{\widehat{C}_{0}, Q_{-}^{C_{0}}, Q_{+}^{\widehat{C}_{0}}}^{*}\left(\widetilde{D}_{0} \subset \widehat{X}\right) \stackrel{\cong}{\longrightarrow} \mathbb{S} H_{\widehat{C}_{0}, Q_{-}^{C_{0}}, Q_{+}^{\widehat{C}_{0}}}\left(\widetilde{D}_{3} \subset \widehat{X}\right)
$$

is an isomorphism. Also by Proposition 5.1, we have an isomorphism

$$
\mathbb{S} H_{\widehat{C}_{0}, Q_{-}^{C_{0}}, Q_{+}^{\widehat{C}_{0}}}^{*}\left(\widetilde{D}_{3} \subset \widehat{X}\right) \cong \mathbb{S} H_{\widehat{C}_{3}, Q_{-}^{\widehat{C}_{0}}, Q_{+}^{\widehat{C}_{0}}}\left(\widetilde{D}_{3} \subset \widehat{X}\right) .
$$

Define

$$
Z:=S H_{\check{C}_{3}, Q_{-}, Q_{+}}^{*}\left(D_{3} \subset X\right) .
$$

Since $\Phi\left(D_{3}\right)=\widetilde{D}_{3}$ and since all 1-periodic orbits and Floer trajectories used to define $Z$ can be made to avoid $V_{X}$ and $V_{\widehat{X}}$ by the ideas in Section 9 due to the fact that they are unions of submanifolds of real codimension $\geq 4$, we have an isomorphism of $\Lambda_{\mathbb{K}}^{\omega_{X}, \omega_{\widehat{X}}}=\Lambda_{\mathbb{K}}^{Q_{+}}$-algebras

$$
Z \cong S H_{\widehat{C}_{3}, Q_{-}, Q_{+}}^{*}\left(\widetilde{D}_{3} \subset \widehat{X}\right)
$$

Since both $\Lambda_{\mathbb{K}}^{\omega_{X}}$ and $\Lambda_{\mathbb{K}}^{\omega_{\widehat{X}}}$ are flat $\Lambda_{\mathbb{K}}^{\omega_{X}, \omega_{\widehat{X}}}$-modules by Proposition 11.2, we have by Theorem 5.39 combined with Equations (7.6), (7.8), (7.9), (7.10) and (7.11) isomorphisms

$$
\begin{aligned}
& Z \otimes_{\Lambda_{\mathbb{K}}^{\omega_{X}, \omega_{\widehat{X}}}} \Lambda_{\mathbb{K}}^{\omega_{X}} \cong S H_{\check{C}_{3}, Q_{-}^{\check{C}_{3}}, Q_{+}^{\check{C}_{3}}}^{*}\left(D_{3} \subset X\right), \\
& Z \otimes_{\Lambda_{\mathbb{K}}^{\omega_{X}, \omega_{\widehat{X}}}} \Lambda_{\mathbb{K}}^{\omega_{\widehat{X}}} \cong S H_{\widehat{C}_{3}, Q_{-}^{\widehat{C}_{3}}, Q_{+}^{\widehat{C}_{3}}}^{*}\left(\widetilde{D}_{3} \subset \widehat{X}\right) .
\end{aligned}
$$

Therefore our theorem now follows from Equations (7.12), (7.13), (7.5), (7.7), (7.9) and (7.10).

\section{Appendix A: Hamiltonians and Almost Complex Structures Compatible with Contact Cylinders.}

This section contains some lemmas allowing us to perturb Hamiltonians so that they become non-degenerate, while retaining certain properties. It also has a lemma telling us that a certain action spectrum has measure 0 .

Definition 8.1. Let $H$ be a Hamiltonian on $M$. Let $\Gamma$ be a collection of 1-periodic orbits of $H$. The associated fixed points of $\Gamma$ is a subset of $M$ denoted by

$$
\Gamma(0):=\{x \in M: \exists \gamma \in \Gamma \text { such that } x=\gamma(0)\} .
$$

We say that $\Gamma$ is isolated if there is a neighborhood $N_{\Gamma}$ of $\Gamma(0)$ so that for each 1-periodic orbit $\gamma$ satisfying $\gamma(0) \in N_{\Gamma}$, we have $\gamma \in \Gamma$. We call $N_{\Gamma}$ an isolating neighborhood of $\Gamma$.

Lemma 8.2. Let $H=\left(H_{t}\right)_{t \in \mathbb{T}}$ be a Hamiltonian on $M$ and let $\Gamma$ be a set of 1-periodic orbits of $M$ which is isolated with isolating neighborhood $N_{\Gamma}$ and let $N$ be a neighborhood of $\Gamma(0)$ whose closure is contained in $N_{\Gamma}$. Let $\mathcal{H}(N, H)$ be the space of Hamiltonians $K=\left(K_{t}\right)_{t \in \mathbb{T}}$ on $M$ satisfying $\left.H_{t}\right|_{\phi_{t}^{H}(N)}=\left.K_{t}\right|_{\phi_{t}^{H}(N)}$ for all $t \in[0,1]$ equipped with the $C^{\infty}$ topology and let $\mathcal{H}^{\mathrm{reg}}(N, H) \subset \mathcal{H}(N, H)$ be the subset of those Hamiltonians with the property that every 1periodic orbit $\gamma$ not in $\Gamma$ is non-degenerate. Then there exists a sequence $\left(H_{i}\right)_{i \in \mathbb{N}}$ of elements in $\mathcal{H}^{\mathrm{reg}}(N, H)$ converging to $H$. 
Proof. Let $N^{\prime} \subset N_{\Gamma}$ be an open set so that $\bar{N} \subset N^{\prime}$ and $\overline{N^{\prime}} \subset N_{\Gamma}$. By [HS95, Theorem 3.1], there is a sequence of non-degenerate Hamiltonians $\left(\check{H}_{i}\right)_{i \in \mathbb{N}} C^{\infty}$ converging to $H$ where $\check{H}_{i}=\left(\check{H}_{i, t}\right)_{t \in \mathbb{T}}$ for all $i \in \mathbb{N}$. Let $\rho: M \longrightarrow[0,1]$ be a smooth function equal to 0 inside $N$ and 1 outside $N^{\prime}$. Define $H_{i, t}:=\left(\phi_{t}^{H}\right)_{*}(\rho) \check{H}_{i, t}+\left(1-\left(\phi_{t}^{H}\right)_{*}(\rho)\right) H_{t}$ for all $t \in \mathbb{T}$ and define $H_{i}:=\left(H_{i, t}\right)_{t \in \mathbb{T}}$. By a compactness argument, we have for all $i$ sufficiently large that any 1periodic orbit $\gamma$ of $H_{i}$ satisfying $\gamma(t) \in \phi_{t}^{H}\left(N^{\prime}\right)$ for some $t \in \mathbb{T}$ also satisfies $\gamma \in \Gamma$. Therefore all 1-periodic orbits $\gamma$ of $H_{i}$ not contained in $\Gamma$ satisfy $\gamma(t) \notin \phi_{t}^{H}\left(N^{\prime}\right)$ for each $t \in \mathbb{T}$ and hence are non-degenerate orbits of $\check{H}_{i, t}$ for all $i$ sufficiently large. Therefore such orbits are non-degenerate orbits of $H_{i, t}$ for all $i$ sufficiently large.

Lemma 8.3. Let $\check{C}$ be a contact cylinder and let $\left(a_{-}, a_{+}\right)$be a $\check{C}$-action interval. Then $\mathcal{H}^{\mathbb{T}, \text { reg }}\left(\check{C}, a_{-}, a_{+}\right)$is an open dense subset of $\mathcal{H}^{\mathbb{T}}\left(\check{C}, a_{-}, a_{+}\right)$(See Definition 2.58).

Proof. Openness is clear, so we just need to prove density. Let $\mathcal{H}^{\prime}\left(\check{C}, a_{-}, a_{+}\right) \subset \mathcal{H}^{\mathbb{T}}\left(\check{C}, a_{-}, a_{+}\right)$ be the subset consisting of Hamiltonians whose average slope along $\check{C}$ is not in the period spectrum of $\check{C}$. This is a dense subset since the period spectrum of both contact cylinders has measure 0 in $\mathbb{R}$ (see [Pop93, Proposition 3.2]).

Now there are two cases to consider. The first case is when $\left(a_{-}, a_{+}\right)$is small and the second case is when $\left(a_{-}, a_{+}\right)$is not small. In the first case, $\mathcal{H}^{\prime}\left(\check{C}, a_{-}, a_{+}\right) \cap \mathcal{H}^{\mathbb{T}, \operatorname{reg}}\left(\check{C}, a_{-}, a_{+}\right)$ is dense in $\mathcal{H}^{\prime}\left(\check{C}, a_{-}, a_{+}\right)$by Lemma 8.2 applied to each $H \in \mathcal{H}^{\prime}\left(\check{C}, a_{-}, a_{+}\right)$with $\Gamma=\emptyset$ and $N_{\Gamma}, N$ small neighborhoods of $[1+\epsilon / 8,1+\epsilon / 2] \times C$ containing no fixed points of $\phi_{1}^{H}$. Hence our lemma is true if $\left(a_{-}, a_{+}\right)$is small.

Now suppose $\left(a_{-}, a_{+}\right)$is not small. Let $D \subset M$ be the Liouville domain associated to $\check{C}$. Then $\mathcal{H}^{\prime}\left(\check{C}, a_{-}, a_{+}\right) \cap \mathcal{H}^{\mathbb{T}, \text { reg }}\left(\check{C}, a_{-}, a_{+}\right)$is dense in $\mathcal{H}^{\prime}\left(\check{C}, a_{-}, a_{+}\right)$by applying Lemma 8.2 to each $H \in \mathcal{H}^{\prime}\left(\check{C}, a_{-}, a_{+}\right)$with $\Gamma=M-(D \cup \check{C})$ and $N, N_{\Gamma}$ a small neighborhood of $\Gamma \cup([1+\epsilon / 8,1+\epsilon / 2] \times C)$ combined with the fact that the capped 1-periodic orbits whose associated 1-periodic orbit has image in $\Gamma$ are not contained in $\Gamma_{\tilde{C}, a_{-}, a_{+}}^{\mathbb{Z}}(H)$ by Equation (2.16) combined with Equation (2.6).

Definition 8.4. Let $H$ be a Hamiltonian. The action spectrum of $H$ is the set:

$$
\left\{\mathcal{A}_{H, \emptyset}(\gamma)([\omega], 1,1): \gamma \text { is a capped 1-periodic orbit of } H\right\}
$$

(see Example 2.11).

Lemma 8.5. The action spectrum of any Hamiltonian is closed and has measure 0.

Proof. We will first prove that the action spectrum of a Hamiltonian $H$ has measure 0. Let $\Delta \subset M \times M$ be the diagonal and let $N \subset M \times M$ be a neighborhood of $\Delta$ so that $(N,-\omega \oplus \omega)$ is symplectomorphic to a neighborhood of $\Delta$ in $T^{*} \Delta$ where such a symplectomorphism is the identity along $\Delta$ (see [MS98, Theorem 3.33]). Let $\lambda$ be the canonical 1-form on $T^{*} \Delta$ and let $\lambda_{N}$ be its restriction to $N$. Let $\gamma$ be a capped 1-periodic orbit of $H$ with associated 1-periodic orbit $\widehat{\gamma}$ and let $U \subset M$ be a simply connected open neighborhood of $\widehat{\gamma}(0)$ so that the graph $\Gamma$ of $\left.\phi_{1}^{H}\right|_{U}$ in $M \times M$ is contained in $N$ and so that $\Gamma$ is transverse to the fibers of $T^{*} \Delta$ (this can be done after perturbing the fibration $T^{*} \Delta$ by a small Hamiltonian flow fixing $\Delta$ ). Then since $\phi_{1}^{H}$ is a symplectomorphism and $U$ is simply connected, there is a unique smooth function $f: \Gamma \longrightarrow \mathbb{R}$ so that

- $f(\widehat{\gamma}(0), \widehat{\gamma}(0))=0$,

- $d f=\left.\lambda_{N}\right|_{\Gamma}$.

Let $O_{U}$ be the set of capped 1-periodic orbits of $H$ whose associated 1-periodic orbit starts in $U$. Then each orbit in $O_{U}$ starts at a critical point of $f$. Let $C_{U} \subset \mathbb{R}$ be the set of critical 
values of $f$. Then for any $\gamma_{0} \in O_{U}$ we have that $\mathcal{A}_{H, \emptyset}\left(\gamma_{0}\right)([\omega], 1,1)=-f\left(\widehat{\gamma}_{0}(0), \widehat{\gamma}_{0}(0)\right)+$ $\mathcal{A}_{H, \emptyset}(\gamma)([\omega], 1,1)+k$ for some $k \in \mathbb{Z}$ (this is because the de Rham cohomology class of $\omega$ admits an integral lift). As a result, every capped 1-periodic orbit of $H$ in $O_{U}$ has action contained in a translation of $\cup_{k \in \mathbb{Z}}\left(C_{U}+k\right)$ which has measure 0 by Sard's theorem. Since the set of fixed points of $\phi_{1}^{H}$ can be covered by a finite number of such subsets $U$, we get that the action spectrum of $H$ has measure 0 .

Now we wish to show that the action spectrum of $H$ is closed. Let $a_{i}$ be a sequence of points in the action spectrum of $H$ which converge to $a \in \mathbb{R}$. Since all 1-periodic orbits of $H$ are contained in a compact set we have a 1-periodic orbit $\widehat{\gamma}_{\infty}$ of $H$ and a sequence of capped 1-periodic orbits $\left(\gamma_{i}\right)_{i \in \mathbb{N}}=\left(\widetilde{\gamma}_{i}, \check{\gamma}_{i}\right)_{i \in \mathbb{N}}$ so that $\mathcal{A}_{H, \emptyset}\left(\gamma_{i}\right)([\omega], 1,1)=a_{i}$ and so that $\widetilde{\gamma}_{i} \circ \check{\gamma}$ $C^{\infty}$ converges to $\widehat{\gamma}_{\infty}$. Since the actions of these capped orbits $\left(\gamma_{i}\right)_{i \in \mathbb{N}}$ are bounded and since their associated 1-periodic orbits converge to $\widehat{\gamma}_{\infty}$, there is a capped 1-periodic orbit $\gamma_{\infty}$ and a sequence of $H_{2}(M ; \mathbb{Z})$ classes $\left(v_{i}\right)_{i \in \mathbb{N}}$ satisfying $\omega\left(v_{i}\right)=0$ for all $i \in \mathbb{N}$ so that $\left(\gamma_{i} \# v_{i}\right)_{i \in \mathbb{N}}$ converges to $\gamma_{\infty}$ in the natural topology on capped loops as described in Definition 2.1 and where \# is given in Definition 2.61. Hence $\left(a_{i}\right)_{i \in \mathbb{N}}$ converges to $\mathcal{A}_{H, \emptyset}\left(\gamma_{\infty}\right)([\omega], 1,1)=a$. Hence the action spectrum is closed.

\section{Appendix B: Avoiding Codimension $\geq 4$ Submanifolds.}

In this section we will show that families of Floer trajectories intersect any countable collection of submanifolds transversely. Also since we are working in the semi-positive setting, we also need low dimensional families of such Floer trajectories to avoid holomorphic spheres (this is needed for compactness to prevent bubbling). These are standard arguments whose main ideas are contained in [MS04, Section 6.3 and 6.7] for instance. We will mainly cite and use the Floer theoretic machinery developed in [Sch95]. Throughout this section, we will fix:

- a (possibly empty) contact cylinder $\check{C}=[1-\epsilon, 1+\epsilon] \times C \subset M$ (Definition 2.3),

- a Riemann surface $\Sigma$ with $n_{-}$negative cylindrical ends and $n_{+}$positive cylindrical ends labeled by finite sets $I_{-}$and $I_{+}$respectively (Definition 2.13),

- a $\Sigma$-compatible 1 -form $\beta \in \Omega^{1}(\Sigma)$ with weights $\left(\kappa_{j}\right)_{j \in I_{-} \sqcup I_{+}}$at the corresponding cylindrical ends (Definition 2.14),

- a $(\Sigma, \check{C})$-compatible family of Hamiltonians $H:=\left(H_{\sigma}\right)_{\sigma \in \Sigma}$ with limits $H^{\#}:=\left(H^{j}\right)_{j \in I_{-} \sqcup I_{+}}$ as in Definition 2.14,

- a tuple $J^{\#}:=\left(J^{j}\right)_{j \in I_{-} \sqcup I_{+}}$of elements of $\mathcal{J}^{\mathbb{T}}(\check{C})$ (Definition 2.12) and

- $\Gamma(H)$ the set of tuples $\left(\gamma^{j}\right)_{j \in I_{-} \sqcup I_{+}}$of non-degenerate capped 1-periodic orbits of $\left(\kappa_{j} H^{j}\right)_{j \in I_{-} \sqcup I_{+}}$respectively whose associated 1-periodic orbits are disjoint from $V$ so that if $\Sigma \neq \mathbb{R} \times \mathbb{T}$ then at least two such 1-periodic orbits have distinct images (Definition 2.1).

Definition 9.1. ([Sch95, Definition 2.1.2]) Let $\bar{\Sigma}$ be a manifold with boundary obtained by extending each cylindrical end $\iota_{i}: \mathbb{I}_{-} \times \mathbb{T} \hookrightarrow \Sigma$ (resp. $\iota_{i}: \mathbb{I}_{+} \times \mathbb{T} \hookrightarrow \Sigma$ ) of $\Sigma$ to $\bar{\iota}_{i}:[-\infty, 0] \times \mathbb{T} \hookrightarrow \bar{\Sigma}, i \in I_{-}$(resp. $\bar{\iota}_{i}:[0, \infty] \times \mathbb{T} \longrightarrow \Sigma, i \in I_{+}$). We get a smooth structure on this manifold by extending the smooth charts

$$
\begin{gathered}
\operatorname{Im}\left(\iota_{i}\right) \longrightarrow(-1,0] \times \mathbb{T}, \quad(s, t) \longrightarrow\left(\frac{s}{\sqrt{1+s^{2}}}, t\right), i \in I_{-} \\
\operatorname{Im}\left(\iota_{i}\right) \longrightarrow[0,1) \times \mathbb{T}, \quad(s, t) \longrightarrow\left(\frac{s}{\sqrt{1+s^{2}}}, t\right), i \in I_{+}
\end{gathered}
$$

on $\Sigma$ to charts

$$
\operatorname{Im}\left(\iota_{i}\right) \longrightarrow[-1,0] \times \mathbb{T}, \quad \operatorname{Im}\left(\iota_{i}\right) \longrightarrow[0,1] \times \mathbb{T}
$$


respectively on $\bar{\Sigma}$.

Definition 9.2. ([Sch95, Definition 2.1.5]) Let $k=0$ or 1. Let $\pi: E \longrightarrow \bar{\Sigma}$ be a $C^{k}$ vector bundle and let $\phi_{i}: \iota_{i}^{*} E \longrightarrow\left(\mathbb{I}_{ \pm} \times \mathbb{T}\right) \times \mathbb{R}^{k}$ be a $C^{k}$ trivialization which extends to a $C^{k}$ trivialization of $\tau_{i}^{*} E$ and let $\Pi_{i}:\left(\mathbb{I}_{ \pm} \times \mathbb{T}\right) \times \mathbb{R}^{k} \rightarrow \mathbb{R}^{k}$ be the natural projection map for each $i \in I_{ \pm}$. Define

$$
W_{\Sigma}^{k, p}(E):=\left\{\sigma \in W_{\mathrm{loc}}^{k, p}\left(\left.E\right|_{\Sigma}\right): \Pi_{i} \circ \phi_{i} \circ \sigma \circ \iota_{i} \in W^{k, p}\left(\mathbb{I}_{ \pm} \times \mathbb{T}, \mathbb{R}^{k}\right), \forall i \in I_{ \pm}\right\} .
$$

We define $L_{\Sigma}^{p}(E):=W_{\Sigma}^{0, p}(E)$. If $D \subset E$ is a subset of $E$ then we define $W_{\Sigma}^{k, p}(D):=\{\sigma \in$ $\left.W_{\Sigma}^{k, p}(E): \operatorname{Im}(\sigma) \subset D\right\}$ and $L_{\Sigma}^{p}(D):=W_{\Sigma}^{0, p}(D)$.

These are Banach spaces which do not depend on the choice of trivializations $\phi_{i}, i \in I_{-} \sqcup I_{+}$ by the paragraph after [Sch95, Definition 2.1.5].

Definition 9.3. ([Sch95, Definition 2.1.6]). Let $\gamma:=\left(\gamma_{i}\right)_{i \in I_{-} \sqcup I_{+}}=\left(\left(\widetilde{\gamma}_{i}, \check{\gamma}_{i}\right)\right)_{i \in I_{-} \sqcup I_{+}}$be finite collection of capped loops as in Definition 2.1. A smooth map $u: \bar{\Sigma} \longrightarrow M$ converges to $\left(\gamma_{i}\right)_{i \in I_{-} \sqcup I_{+}}$if $\iota_{i}( \pm \infty, t)=\widetilde{\gamma}_{i}\left(\check{\gamma}_{i}(t)\right)$ for all $i \in I_{ \pm}, t \in \mathbb{T}$ and the surface obtained by gluing the surfaces $\widetilde{\gamma}_{i}, i \in I_{-} \sqcup I_{+}$to $u$ is null-homologous. Let $C_{\gamma}^{\infty}(\bar{\Sigma}, M)$ be the space of such maps equipped with the $C^{\infty}$ topology.

Fix a Riemannian metric on $M$ and let $D \subset T M$ be an open neighborhood of $M$ so that $\left.\exp \right|_{D \cap T_{x} M}$ is a diffeomorphism on to its image for all $x \in M$ where exp is the exponential map with respect to this metric. Define

$$
\mathcal{P}_{\gamma}^{1, p}(\Sigma, M):=\left\{\exp \circ v \in C^{0}(\bar{\Sigma}, M): v \in W_{\Sigma}^{1, p}\left(h^{*} D\right), h \in C_{\gamma}^{\infty}(\bar{\Sigma}, M)\right\}
$$

for all $p>2$.

The space (9.1) is a Banach manifold by [Sch95, Theorem 2.1.7] with charts mapping to $W_{\Sigma}^{1, p}\left(h^{*} D\right)$ for all $h \in C_{\gamma}^{\infty}(\bar{\Sigma}, M)$.

Definition 9.4. Let $\pi: E \longrightarrow B$ be a Banach vector bundle. Let $s: B \longrightarrow E$ be a $C^{1}$ section and let $b \in s^{-1}(0)$, We say that $s$ is transverse to zero at $b$ if the linear map

$$
\left.T_{b} B \stackrel{s}{\longrightarrow} T_{s(b)} E \stackrel{\mathrm{pr}}{\longrightarrow} \operatorname{ker} D \pi\right|_{s(b)}
$$

is surjective where

$$
\text { pr: }\left.T E\right|_{B}=\left.T B \oplus\left(\left.\operatorname{ker} D \pi\right|_{B}\right) \longrightarrow \operatorname{ker} D \pi\right|_{B}
$$

is the natural projection map. We say that $s$ is transverse to 0 if it is transverse to 0 at every point $b \in s^{-1}(0)$. Such a section is Fredholm if the map (9.2) is Fredholm.

Definition 9.5. For each $J=\left(J_{\sigma}\right)_{\sigma \in \Sigma} \in \mathcal{J}^{\Sigma}\left(J^{\#}, \check{C}\right)$, we define $M^{J}$ to be the vector bundle over $\bar{\Sigma} \times M$ whose fiber at $(\sigma, x)$ is the space of $\left.J\right|_{(\sigma, x)}$ anti-linear maps from $T_{\sigma} \bar{\Sigma}$ to $T_{x} M$.

Proposition 9.6. [Sch95, Theorem 2.2.5, Proposition 2.3.1, Theorem 3.1.31]. Let $\gamma \in \Gamma(H)$ and $J=\left(J_{\sigma}\right)_{\sigma \in \Sigma} \in \mathcal{J}^{\Sigma}\left(J^{\#}, \check{C}\right)$. Then the set

$$
\mathcal{E}_{J, \gamma}:=\sqcup_{u \in \mathcal{P}_{\gamma}^{1, p}(\Sigma, M)}\{u\} \times L_{\Sigma}^{p}\left(\left(\mathrm{id}_{\bar{\Sigma}}, u\right)^{*} M^{J}\right)
$$

is naturally a smooth Banach bundle over $\mathcal{P}_{\gamma}^{1, p}(\Sigma, M)$ for all $p>2$. Also if $\mathbf{j}$ is the natural complex structure on $\Sigma$ then the section

$$
\begin{gathered}
\bar{\partial}_{J, \gamma}: \mathcal{P}_{\gamma}^{1, p}(\Sigma, M) \longrightarrow \mathcal{E}_{J, \gamma}, \\
\bar{\partial}_{J, \gamma}(u)(\sigma):=\left(d u+X_{H_{\sigma}} \otimes \beta\right)+J_{\sigma} \circ\left(d u+X_{H_{\sigma}} \otimes \beta\right) \circ \mathbf{j}, \quad \forall \sigma \in \Sigma
\end{gathered}
$$


is well defined (I.e. $\bar{\partial}_{J, \gamma}(u)$ is an element of $L_{\Sigma}^{p}\left(\left(\mathrm{id}_{\bar{\Sigma}}, u\right)^{*} M^{J}\right)$ for all $\left.u \in \mathcal{P}\right)$ and $\bar{\partial}_{J, \gamma}$ is a smooth Fredholm section of $\mathcal{E}_{J, \gamma}$.

Definition 9.7. An element $J \in \mathcal{J}^{\Sigma}\left(J^{\#}, \check{C}\right)$ is $H$-regular if the Fredholm section $\bar{\partial}_{J, \gamma}$ is transverse to zero for each $\gamma \in \Gamma(H)$.

Note that if the Hamiltonian $H$ only has degenerate one periodic orbits then every element $J \in \mathcal{J}^{\Sigma}\left(J^{\#}, \check{C}\right)$ is $H$-regular by the definition above since $\Gamma(H)$ is empty. We only care about Floer trajectories connecting non-degenerate 1-periodic orbits.

Theorem 9.8. [Sch95, Theorem 4.2 .2 and 3.3.11]. If $J \in \mathcal{J}^{\Sigma}\left(J^{\#}, \check{C}\right)$ is $H$-regular then $\mathcal{M}(H, J, \gamma)=\bar{\partial}_{J, \gamma}^{-1}(0)$ and this set is a smooth finite dimensional submanifold of $\mathcal{P}_{\gamma}^{1, p}(\Sigma, M)$ of dimension $k$ where $k$ is defined in Equation (2.9) for each $\gamma \in \Gamma(H)$.

Definition 9.9. Let $f_{j}: W_{j} \longrightarrow W, j=0,1$ be two smooth maps. We say that $f_{0}$ is transverse to $f_{1}$ if, for each $w_{0} \in W_{0}, w_{1} \in W_{1}$ satisfying $f_{0}\left(w_{0}\right)=f_{1}\left(w_{1}\right)$, we have that the span of the subspaces $D f_{0}\left(T_{w_{0}} W_{0}\right), D f_{1}\left(T_{w_{1}} W_{1}\right)$ is $T_{f_{0}\left(w_{0}\right)} W$.

Definition 9.10. For each $\gamma \in \Gamma(H)$, we say that $J=\left(J_{\sigma}\right)_{\sigma \in \Sigma} \in \mathcal{g}^{\Sigma}\left(J^{\#}, \check{C}\right)$ is $(H, V, \gamma)$ regular if it is $H$-regular and if there is a countable collection of smooth maps $f_{i}: W_{i} \longrightarrow$ $\Sigma \times M, i \in \mathbb{N}$ where $\operatorname{dim}_{\mathbb{R}}\left(W_{i}\right) \leq 2 n-2$ for each $i$ so that

(1) $V \subset \cup_{i \in \mathbb{N}} f_{i}\left(W_{i}\right)$,

(2) every $J_{\sigma}$-holomorphic map $u: \mathbb{P}^{1} \longrightarrow M$ has image contained in $(\{\sigma\} \times M) \cap$ $\left(\cup_{i} f_{i}\left(W_{i}\right)\right)$ after identifying $\{\sigma\} \times M$ with $M$ in the natural way for each $\sigma \in \Sigma$ and

(3) the evaluation map

$$
\text { ev }: \Sigma \times \mathcal{M}(H, J, \gamma) \longrightarrow \Sigma \times M, \quad(\sigma, u) \longrightarrow(\sigma, u(\sigma))
$$

is smooth and transverse to $f_{i}$ for each $i \in \mathbb{N}$.

We say that $J$ is $(H, V)$-regular if it is $(H, V, \gamma)$-regular for each $\gamma \in \Gamma(H)$. We define $\mathcal{J}^{\Sigma, \operatorname{reg}}\left(H, J^{\#}, \check{C}\right) \subset \mathcal{J}\left(J^{\#}, \check{C}\right)$ to be the subspace of $(H, V)$-regular families of almost complex structures.

We wish to show that $\mathcal{J}^{\Sigma, \text { reg }}\left(H, J^{\#}, \check{C}\right)$ is ubiquitous in $\mathcal{J}\left(J^{\#}, \check{C}\right)$ (Proposition 2.19). In order to do this we need to define an appropriate space of almost complex structures.

Definition 9.11. [Sch95, Definitions 4.2.6, 4.2.10 and 4.2.11]. Let $J \in \mathcal{J}^{\Sigma}\left(J^{\#}, \check{C}\right)$. Let $U \subset \Sigma$ be a relatively compact open subset, define $\widetilde{U}:=U \times(M-([1+\epsilon / 8,1+\epsilon / 2] \times C))$ and let $\pi: \Sigma \times M \longrightarrow M$ be the natural projection map. Let

$$
S_{J}:=\left\{A \in \operatorname{End}\left(\pi^{*} T M\right): A J+J A=0\right\}
$$

be a bundle over $\Sigma \times M$ and let $C_{U}^{\infty}\left(S_{J}\right)$ be the space of $C^{\infty}$ sections $A$ of $S_{J}$ so that all the derivatives of $A$ vanish along $\Sigma \times V$ and $\Sigma \times M-\widetilde{U}$. Let $\|\cdot\|$ be a metric on $M$ given by $\frac{1}{2}\left(\omega(\cdot, J(\cdot))+\omega(J(\cdot), \cdot)\right.$. Let $\epsilon:=\left(\epsilon_{i}\right)_{i \in \mathbb{N}}$ be a sequence of rapidly decreasing positive constants and define

$$
C_{\epsilon, U}^{\infty}\left(S_{J}\right):=\left\{A \in C_{U}^{\infty}\left(S_{J}\right):\|A\|_{\epsilon}<\infty\right\}
$$

where $\|A\|_{\epsilon}:=\sum_{i \in \mathbb{N}} \epsilon_{i}\left\|\nabla^{k} A\right\|$ coming from a product metric on $\Sigma \times M$. Define

$$
\Phi_{J}: C_{\epsilon, U}^{\infty}\left(S_{J}\right) \longrightarrow \mathcal{J}^{\Sigma}\left(J^{\#}, \check{C}\right), \quad \Phi_{J}(A):=J e^{A}
$$

and define

$$
\mathcal{\partial}_{\epsilon}\left(\left.J\right|_{U}\right):=\left\{\Phi_{J}(A): A \in C_{\epsilon, U}^{\infty}\left(S_{J}\right),\|A\|<\epsilon_{0}\right\} \subset \mathcal{J}^{\Sigma}\left(J^{\#}, \check{C}\right) .
$$


By the ideas in $[S \operatorname{sch} 95,4.2 .7,4.2 .9,4.2 .10]$ we have that $C_{\epsilon, U}^{\infty}\left(S_{J}\right)$ is a Banach space with Banach norm $\|\cdot\|_{\epsilon}$ and $\Phi_{J}^{-1}$ embeds $\mathcal{J}_{\epsilon}\left(\left.J\right|_{U}\right)$ as an open subset of this Banach space making it into a Banach manifold for $\epsilon$ small enough. There are a few minor differences between Definition 9.11 and [Sch95, Definition 4.2.11].

(1) Our almost complex structures are not necessarily compatible with $\omega$, but they do tame $\omega$,

(2) Our almost complex structures and all of their derivatives agree with those of $J$ along some regions of $\Sigma \times M$ and

(3) the formula for the map $\Phi_{J}$ is different.

These differences play no important role in the proof of the fact that $\partial_{\epsilon}\left(\left.J\right|_{U}\right)$ is a Banach manifold. Also it makes no difference in the proofs of Propositions 9.14, 9.16 and 9.18 which are just modified versions of [Sch95, Proposition 4.2.4], [Sch95, 4.2.18] and [Sch95, Proposition 4.2.5] respectively. Now one of the issues with $\mathcal{J}_{\epsilon}\left(\left.J\right|_{U}\right)$ is that it is not a topological subspace of $\mathcal{g}^{\Sigma}\left(J^{\#}, \check{C}\right)$. However we wish to prove theorems with respect to the topology of $\mathcal{g}^{\Sigma}\left(J^{\#}, \check{C}\right)$. The following lemma addresses this issue. If $\epsilon=\left(\epsilon_{i}\right)_{i \in \mathbb{N}}$ and $\epsilon^{\prime}=\left(\epsilon_{i}^{\prime}\right)_{i \in \mathbb{N}}$ are sequences of positive constants, we write $\epsilon \leq \epsilon^{\prime}$ if $\epsilon_{i} \leq \epsilon_{i}^{\prime}$ for all $i \in \mathbb{N}$.

Lemma 9.12. Let $\epsilon^{U, J}$ be a sequence of rapidly decreasing constants for each $J \in \mathcal{J}^{\Sigma}\left(J^{\#}, \check{C}\right)$ and each relatively compact subset $U \subset \Sigma$. Let $W_{U, J, \epsilon} \subset \partial_{\epsilon}\left(\left.J\right|_{U}\right)$ be a ubiquitous subset for each $J \in J^{\Sigma}\left(J^{\#}, \check{C}\right)$, relatively compact open subset $U \subset \Sigma$ and each $\epsilon \leq \epsilon^{U, J}$. Then $\cup_{U, J, \epsilon} W_{U, J, \epsilon}$ is ubiquitous in $J^{\Sigma}\left(J^{\#}, \check{C}\right)$.

Proof. Choose a sequence $U_{i} \subset \Sigma, i \in \mathbb{N}$ of relatively compact open subsets whose union is $\Sigma$. Choose a sequence of constants $\epsilon^{J, i} \leq \epsilon^{J, U_{i}}, i \in \mathbb{N}$ and an open neighborhood $W_{J} \subset \mathcal{J}^{\Sigma}\left(J^{\#}, \check{C}\right)$ of $J$ so that $V_{J}:=\cup_{i \in \mathbb{N}} \mathcal{J}_{\epsilon^{J, i}}\left(\left.J\right|_{U_{i}}\right)$ contains $W_{J}$ for each $J \in \mathcal{J}^{\Sigma}\left(J^{\#}, \check{C}\right)$. Choose an open neighborhood $\check{W}_{J} \subset \mathcal{J}^{\Sigma}\left(J^{\#}, \check{C}\right)$ of $J$ whose closure is contained in $W_{J}$ for each $J \in \mathcal{J}^{\Sigma}\left(J^{\#}, \check{C}\right)$. Separability of $\mathcal{J}^{\Sigma}\left(J^{\#}, \check{C}\right)$ implies that there exists a sequence $\left(J_{i}\right)_{i \in \mathbb{N}}$ of elements in $\mathcal{J}^{\Sigma}\left(J^{\#}, \check{C}\right)$ so that $\mathcal{J}^{\Sigma}\left(J^{\#}, \check{C}\right)=\cup_{i \in \mathbb{N}} \check{W}_{J_{i}}$. Define

$$
W^{i, j}:=W_{U_{j}, J_{i}, \epsilon^{J_{i}, j}} \cup\left(\mathrm{g}^{\Sigma}\left(J^{\#}, \check{C}\right)-\overline{W_{J}}\right) \subset \mathrm{g}^{\Sigma}\left(J^{\#}, \check{C}\right) .
$$

Then $W^{i, j}$ is ubiquitous in $\mathcal{J}^{\Sigma}\left(J^{\#}, \check{C}\right)$ and $W^{i, j} \cap \mathcal{J}_{\epsilon_{i} J_{i}, j}\left(\left.J_{i}\right|_{U_{j}}\right) \cap \check{W}_{J_{i}}=W_{U_{j}, J_{i}, \epsilon^{J_{i}, j}} \cap \check{W}_{J_{i}}$ for each $i, j \in \mathbb{N}$. Hence $\cap_{i, j \in \mathbb{N}} W^{i, j}$ is ubiquitous in $\mathcal{g}^{\Sigma}\left(J^{\#}, \check{C}\right)$ and is contained in $\cup_{U, J, \epsilon} W_{U, J, \epsilon}$ and therefore $\cup_{U, J, \epsilon} W_{U, J, \epsilon}$ is ubiquitous.

By combining Lemma 9.12 above with [Sch95, Proposition 4.2.5] (see also Proposition 9.16 below), we get the following proposition:

Proposition 9.13. The subspace of $H$-regular almost complex structures in $\mathcal{J}^{\Sigma}\left(J^{\#}, \check{C}\right)$ as in Definition 9.7 is ubiquitous.

We now wish to prove the same thing for $(H, V)$-regular almost complex structures. In order to do this we need some more propositions and lemmas. Note that every element $J=\left(J_{\sigma}\right)_{\sigma \in \Sigma}$ in $g^{\Sigma}\left(J^{\#}, \check{C}\right)$ extends to a smooth family of almost complex structures $\left(J_{\sigma}\right)_{\sigma \in \bar{\Sigma}}$ since $J$ is translation invariant in the cylindrical ends at infinity. Hence, from now on we will define $J_{\sigma}$ to be the limit as $\sigma^{\prime} \in \Sigma$ tends to $\sigma$ of $J_{\sigma^{\prime}}$ for each $\sigma \in \partial \bar{\Sigma}$. We will also use such conventions for other families of objects over $\Sigma$ such as $\beta$ and $H$.

Proposition 9.14. [Sch95, Proposition 4.2.4 and Theorem 3.1.31]. Let $U \subset \Sigma$ be a relatively compact open set, $\epsilon$ a sequence of rapidly decreasing constants, $J \in \mathcal{J}^{\Sigma}\left(J^{\#}, \check{C}\right)$ and $\gamma \in \Gamma(H)$. 
Let $\mathcal{B}:=\mathcal{J}_{\epsilon}\left(\left.J\right|_{U}\right)$ and $\mathcal{P}:=\mathcal{P}_{\gamma}^{1, p}(\Sigma, M), p>2$ where $\mathcal{P}_{\gamma}^{1, p}(\Sigma, M)$ is defined as in Equation (9.1). Then the set

$$
\mathcal{E}:=\sqcup_{\left(J^{\prime}, u\right) \in \mathcal{B} \times \mathcal{P}}\left\{\left(J^{\prime}, u\right)\right\} \times L_{\Sigma}^{p}\left(\left(\operatorname{id}_{\bar{\Sigma}}, u\right)^{*} M^{J^{\prime}}\right)
$$

is naturally a smooth Banach bundle over $\mathcal{B} \times \mathcal{P}$ whose fiber over $\left(J^{\prime}, u\right)$ is $L_{\Sigma}^{p}\left(\left(\mathrm{id}_{\bar{\Sigma}}, u\right)^{*} M^{J^{\prime}}\right)$. Also

$$
\bar{\partial}_{\mathcal{B} \times \mathcal{P}}: \mathcal{B} \times \mathcal{P} \longrightarrow \mathcal{E}, \quad \bar{\partial}_{\mathcal{B} \times \mathcal{P}}\left(J^{\prime}, u\right):=\bar{\partial}_{J^{\prime}, \gamma}(u)
$$

is a smooth section of $\mathcal{E}$ where $\bar{\partial}_{J, \gamma}$ is defined in Equation (9.4).

From now on, until the proof of Proposition 2.19 below, we will fix $U, \epsilon, J, \gamma, \mathcal{B}, \mathcal{P}, \mathcal{E}$ and $\bar{\partial}_{\mathcal{B} \times \mathcal{E}}$ from the proposition above.

Definition 9.15. Define $\mathcal{S} \subset \mathcal{B} \times \mathcal{P}$ to be the open subset of pairs $\left(J^{\prime}, u\right) \in \mathcal{B} \times \mathcal{P}$ satisfying

$$
U \nsubseteq u^{-1}(V \cup([1+\epsilon / 8,1+\epsilon / 2] \times C)) \cup\left(\Sigma-\operatorname{supp}\left(d u-\beta \otimes X_{H}\right)\right)
$$

where $\operatorname{supp}\left(d u-\beta \otimes X_{H}\right)$ is the support of the distribution $d u-X_{H} \otimes \beta \in L_{\mathrm{loc}}^{p}\left(\Sigma \otimes u^{*} T M\right)$ where

$$
\left.X_{H} \otimes \beta\right|_{\sigma}:=\left.\left.X_{H_{\sigma}}\right|_{u(\sigma)} \otimes \beta\right|_{\sigma} \forall \sigma \in \Sigma .
$$

We also define $\mathcal{D}:=\bar{\partial}_{\mathcal{B} \times \mathcal{P}}^{-1}(0) \cap \mathcal{S}$ and let

$$
\Pi_{\mathcal{B}}: \mathcal{D} \longrightarrow \mathcal{B} .
$$

be the natural projection map.

Proposition 9.16. [Sch95, Proposition 4.2.18 and its proof]. Suppose that $\left(J^{\prime}, u\right) \in \mathcal{D}$. Then the section $\bar{\partial}_{\mathcal{B} \times \mathcal{P}}$ above is transverse to 0 at $\left(J^{\prime}, u\right)$. Also the natural linear map

$$
\left.D_{J^{\prime}, u}\right|_{T_{J^{\prime}} \mathcal{B}}: T_{J^{\prime}} \mathcal{B}=T_{J^{\prime}} \mathcal{B} \times 0 \stackrel{D \bar{\partial}_{\mathcal{B} \times \mathcal{P}}}{\longrightarrow} T_{\left(J^{\prime}, u\right)} \mathcal{E} \stackrel{\mathrm{pr}}{\longrightarrow} L_{\Sigma}^{p}\left(\left(\mathrm{id}_{\bar{\Sigma}}, u\right)^{*} M^{J^{\prime}}\right)
$$

linearizing $\bar{\partial}_{\mathcal{B} \times \mathcal{P}}$ at $\left(J^{\prime}, u\right)$ has dense image.

Hence by combining the proposition above with the last part of Proposition 9.6 and the implicit function theorem, we get the following corollary.

Corollary 9.17. $\mathcal{D}$ is a Banach submanifold of $\mathcal{S}$.

Proposition 9.18. [Sch95, Theorem 3.3.11, Theorem 4.2.2, Proposition 4.2.5 together with its proof]. The map $\Pi_{\mathcal{B}}$ in Equation (9.7) is Fredholm. The subset $\left(\Pi_{\mathcal{B}}\right)^{\text {reg }} \subset \mathcal{B}$ of regular values of $\Pi_{\mathcal{B}}$ is ubiquitous as in Definition 2.18 and $\Pi_{\mathfrak{\gamma}}^{-1}\left(J^{\prime}\right)$ is a smooth manifold of dimension $k$ where $k$ is defined in Equation (2.9) for all $J^{\prime} \in \Pi_{\mathcal{B}}$.

By the Sobolev embedding theorem we can think of the tangent space $T_{u} \mathcal{P}$ at a point $u \in \mathcal{P}$ naturally as a subspace of $C^{0}\left(u^{*} T M\right)$. Let $\iota_{\mathcal{P}}: T_{u} \mathcal{P} \longrightarrow C^{0}\left(u^{*} T M\right)$ be the natural inclusion map. Then we have the following definition:

Definition 9.19. For each $u \in \mathcal{P}$ and $\sigma \in \Sigma$, define the Banach subspace $T_{u, \sigma} \mathcal{P} \subset T_{u} \mathcal{P}$ to be the subspace consisting of elements $v \in T_{u} \mathcal{P}$ satisfying $\iota_{\mathcal{P}}(v)(\sigma)=0$.

Lemma 9.20. Let $\sigma \in \Sigma$ and let $\left(J^{\prime}, u\right) \in \mathcal{D}$. Then the map

$$
D_{J^{\prime}, u, \sigma}: T_{J^{\prime}} \mathcal{B} \times T_{u, \sigma} \mathcal{P} \stackrel{D \overline{\mathcal{B}}_{\mathcal{B} \times \mathcal{P}}}{\longrightarrow} T_{\left(J^{\prime}, u\right)} \mathcal{E} \stackrel{\mathrm{pr}}{\longrightarrow} L_{\Sigma}^{p}\left(\left(\mathrm{id}_{\bar{\Sigma}}, u\right)^{*} M^{J^{\prime}}\right)
$$

is surjective. 
Proof. The map

$$
\left.D_{J^{\prime}, u}\right|_{0 \times T_{u} \mathcal{P}}: 0 \times T_{u} \mathcal{P} \stackrel{D \bar{\partial}_{\mathcal{B} \times \mathcal{P}}}{\longrightarrow} T_{\left(J^{\prime}, u\right)} \mathcal{E} \stackrel{\mathrm{pr}}{\longrightarrow} L_{\Sigma}^{p}\left(\left(\operatorname{id}_{\bar{\Sigma}}, u\right)^{*} M^{J^{\prime}}\right)
$$

is Fredholm by Proposition 9.14 and hence the map $\left.D_{J^{\prime}, u, \sigma}\right|_{0 \times T_{u, \sigma} \mathcal{P}}$ is Fredholm since $T_{u, \sigma} \mathcal{P} \subset$ $T_{u} \mathcal{P}$ is a subspace of finite codimension. This implies that the image of $D_{J^{\prime}, u, \sigma}$ is closed. Such an image is also dense by the last part of Proposition 9.16 and hence is surjective.

Lemma 9.21. The natural map

$$
E: \Sigma \times \mathcal{D} \longrightarrow M, \quad E\left(\sigma,\left(J^{\prime}, u\right)\right):=u(\sigma)
$$

is $C^{\infty}$.

Note that if we extend this map to $\mathcal{B} \times \mathcal{P}$ in the natural way then such a map is not even $C^{1}$.

Proof. By [Sch95, Proposition 2.5.7], we have that $u \in C^{\infty}(\Sigma, M)$ for all $\left(J^{\prime}, u\right) \in \mathcal{D}$. By [MS04, Proposition B.4.9] combined with the Sobolov embedding theorem [MS04, Proposition B.1.11], we have that for each $\left(J^{\prime}, u\right) \in \mathcal{D}$, and each compact codimension 0 submanifold $K \subset \Sigma$, the natural map from $T_{\left(J^{\prime}, u\right)} \mathcal{D}$ to $T_{u} C^{r}(K, M)$ is well defined continuous map between Banach spaces. Since charts on mapping spaces are constructed using the exponential map of a metric on $M$, this implies that the natural map from $\mathcal{D}$ to $C^{r}(K, M)$ is smooth for each compact codimension 0 submanifold $K \subset \Sigma$ and each $r \geq 0$. Therefore, our lemma follows from the fact that the evaluation map

$$
K \times C^{r}(K, M) \longrightarrow M
$$

is $C^{r}$ for all compact codimension 0 submanifolds $K \subset \Sigma$ and all $r \geq 0$ ([?, Page 78]).

Lemma 9.22. Let $\sigma \in \Sigma$. Then the map

$$
\left.E\right|_{\{\sigma\} \times \mathcal{D}}: \mathcal{D} \longrightarrow M,\left.\quad E\right|_{\{\sigma\} \times \mathcal{D}}\left(J^{\prime}, u\right)=u(\sigma)
$$

is a submersion.

Proof. Let $\left(J^{\prime}, u\right) \in \mathcal{D}$ and let $W \in T_{u(\sigma)} M$. Choose $w \in T_{u} \mathcal{P}$ so that $w(\sigma)=W$. Since $D_{J^{\prime}, u, \sigma}$ from Lemma 9.20 is surjective, there exists $\left(Y_{1}, w_{1}\right) \in T_{J^{\prime}} \mathcal{B} \times T_{u, \sigma} \mathcal{P}$ so that $D_{J^{\prime}, u}\left(Y_{1}, w_{1}\right)=D_{J^{\prime}, u}(0, w)$ where $T_{u, \sigma} \mathcal{P}$ is defined in Definition 9.19 and where $D_{J^{\prime}, u}$ is the composition

$$
D_{J^{\prime}, u}: T_{J^{\prime}} \mathcal{B} \times T_{u} \mathcal{P} \stackrel{D \bar{\partial}_{\mathcal{B} \times \mathcal{P}}}{\longrightarrow} T_{\left(J^{\prime}, u\right)} \mathcal{E} \stackrel{\mathrm{pr}}{\longrightarrow} L_{\Sigma}^{p}\left(\left(\mathrm{id}_{\bar{\Sigma}}, u\right)^{*} M^{J^{\prime}}\right) .
$$

Therefore $\left(-Y_{1}, w-w_{1}\right) \in T_{J^{\prime}, u} \mathcal{D}$ and $\left(w-w_{1}\right)(\sigma)=W$ and hence the map $\left.E\right|_{\{\sigma\} \times \mathcal{D}}$ is a submersion.

Lemma 9.23. The map

$$
\text { ev }: \Sigma \times \mathcal{D} \longrightarrow \Sigma \times M, \quad F\left(\sigma,\left(J^{\prime}, u\right)\right):=(\sigma, u(\sigma))
$$

is smooth and a submersion.

Proof. This follows directly from Lemmas 9.21 and 9.22.

Lemma 9.24. Let $f:=\left(f_{i}\right)_{i \in \mathbb{N}}$ be a countable collection of smooth maps $f_{i}: Q_{i} \longrightarrow \Sigma \times M$, $i \in \mathbb{N}$. Let $\partial_{\epsilon}\left(\left.J\right|_{U}, f\right) \subset \mathcal{B}$ be the subset consisting of elements $J^{\prime}$ that are $H$-regular with the property that $\left.\mathrm{ev}\right|_{\Pi_{\mathcal{B}}^{-1}\left(J^{\prime}\right)}$ is transverse to fi for each $i \in \mathbb{N}$ where ev and $\Pi_{\mathcal{B}}$ are defined in Equations (9.9) and (9.7) respectively. Then $\mathcal{J}_{\epsilon}\left(\left.J\right|_{U}, f\right)$ is ubiquitous in $\mathcal{B}$. 
Proof. Let $\widetilde{\Pi}_{i}:=\operatorname{ev}^{*} f_{i}: \widetilde{Q}_{i} \longrightarrow \Sigma \times \mathcal{D}$ be the pullback of $f_{i}$ for each $i \in \mathbb{N}$ (this exists by Lemma 9.23). Since $\Sigma \times M$ and $Q_{i}$ is finite dimensional, we have that $\widetilde{\Pi}_{i}$ is a Fredholm map. Hence the composition $P \circ \widetilde{\Pi}_{i}$ is Fredholm by Proposition 9.18 where $P: \Sigma \times \mathcal{D} \longrightarrow \mathcal{B}$ is the natural projection map. Hence the set of regular values $\mathcal{R}_{i}$ of $P \circ \widetilde{\Pi}_{i}$ is ubiquitous in $\mathcal{B}$. The subset of $H$-regular almost complex structures $\mathcal{J}_{H}^{\text {reg }}$ is ubiquitous by Lemma 9.13. Our lemma now follows from the fact that $\mathcal{J}_{\epsilon}\left(\left.J\right|_{U}, f\right)$ contains the ubiquitous set $\mathcal{J}_{H}^{\mathrm{reg}} \cap \cap_{i \in \mathbb{N}} \mathcal{R}_{i}$.

Proof of Proposition 2.19. Let $S_{i}, U_{i} \subset \Sigma, i \in \mathbb{N}$ be non-empty relatively compact open subsets of $\Sigma$ so that $\cup_{i} S_{i}=\Sigma, S_{i} \subset S_{i+1}$ and $\overline{U_{i}} \cap \overline{S_{i}}=\emptyset$ for all $i \in \mathbb{N}$. For each $J=$ $\left(J_{\sigma}\right)_{\sigma \in \Sigma} \in \mathcal{J}^{\Sigma}\left(J^{\#}, \check{C}\right)$, let $\mathcal{M}^{\text {vert }}(J)$ be the set of somewhere injective maps $v: \mathbb{P}^{1} \longrightarrow \Sigma \times M$ where

(1) the image of $v$ is contained in $\left\{\sigma_{v}\right\} \times M$ for some $\sigma_{v} \in \Sigma$ and not contained in $\Sigma \times V$ and

(2) $v$ is $J_{\sigma_{v}}$-holomorphic after identifying $\left\{\sigma_{v}\right\} \times M$ with $M$ in the natural way.

Then by the methods in [MS04, Section 3.2] we have that there is a ubiquitous subset $\mathcal{J}_{\mathbb{P} 1}^{\text {reg }} \subset$ $\mathcal{J}^{\Sigma}\left(J^{\#}, \check{C}\right)$ so that $\mathcal{M}^{\text {vert }}(J)$ is a manifold whose connected components are of dimension at most $2 n-2$ where $2 n$ is the dimension of $M$ and so that the evaluation map

$$
\operatorname{ev}_{\mathbb{P}^{1}}: \mathcal{M}^{\mathrm{vert}}(J) \longrightarrow \Sigma \times M, \quad \operatorname{ev}_{\mathbb{P}^{1}}(u):=u(0)
$$

is smooth. Then for each $J \in \mathcal{J}_{\mathbb{P} 1}^{\text {reg }}$, there exists a countable collection of smooth maps $f_{J}^{i}: W_{J}^{i} \longrightarrow \Sigma \times M, i \in \mathbb{N}$ where $\operatorname{dim}\left(W_{J}^{i}\right) \leq 2 n-2$ so that for each $u \in \mathcal{M}^{\text {vert }}(J)$, we have that image $(u) \subset \cup_{i \in \mathbb{N}} f_{J}^{i}\left(W_{J}^{i}\right)$. We can assume that the functions $f_{J}^{i}, i \in \mathbb{N}, J \in \mathcal{J}_{\mathbb{P} 1}^{\mathrm{reg}}$ have the property that if there exists $i \in \mathbb{N}$ so that $J=\left(J_{\sigma}\right)_{\sigma \in \Sigma}, J^{\prime}=\left(J_{\sigma}^{\prime}\right)_{\sigma \in \Sigma} \in \mathcal{J}_{\mathbb{P} 1}^{\text {reg }}$ satisfies $J_{\sigma}=J_{\sigma}^{\prime}$ for all $\sigma \in S_{i}$ then $E_{i, k, J}:=\left(f_{J}^{k}\right)^{-1}\left(S_{i} \times M\right)$ is diffeomorphic to $\left(f_{J^{\prime}}^{k}\right)^{-1}\left(S_{i} \times M\right)$ and $\left.f_{J}^{k}\right|_{E_{i, k, J}}=\left.f_{J^{\prime}}^{k}\right|_{E_{i, k, J}}$ under this identification for all $k \in \mathbb{N}$. Since any manifold is a countable union of compact codimension 0 submanifolds with boundary, we can assume that $W_{J}^{i}$ is a compact manifold with boundary for each $i \in \mathbb{N}$ as well.

We now wish to write the moduli spaces $\mathcal{M}(H, J, \gamma)$ as a union of compact sets for each $H, J, \gamma$ in a consistent way. We will use Gromov compactness ideas to do this. For each $j \in I_{ \pm}$and each non-degenerate fixed point $p$ of $\phi_{1}^{\kappa_{j} H^{j}}$, let $N_{j, p}$ be a neighborhood of $p$ whose closure $\overline{N_{j, p}}$ does not contain any other fixed points of $\phi_{1}^{\kappa_{j} H^{j}}$. For each $J \in \mathcal{J}^{\Sigma}\left(J^{\#}, \check{C}\right)$ and each $\gamma=\left(\gamma^{j}\right)_{j \in I_{-} \sqcup I_{+}} \in \Gamma(H)$, let $K(i, J, \gamma) \subset \mathcal{M}(H, J, \gamma)$ be the subset of maps $u: \Sigma \longrightarrow M$ satisfying $|d u| \leq i$ with respect to a fixed metric on $\Sigma$ which is translation invariant on the cylindrical ends and so that $u\left(\iota_{j}(s, t)\right) \in \phi_{t}^{\kappa_{j} H^{j}}\left(\overline{N_{j, \widehat{\gamma}^{j}(0)}}\right)$ for each $\pm s \geq i$ and $t \in \mathbb{T}$ where $\widehat{\gamma}^{j}$ is the 1-periodic associated to $\gamma^{j}$ for each $j \in I_{ \pm}$. Then a Gromov compactness argument (e.g. [Sch95, Theorem 4.3.22]) tells us that for each $J \in \mathcal{J}^{\Sigma}\left(J^{\#}, \check{C}\right)$ and $\gamma \in \Gamma(H), K(i, J, \gamma)$ is compact for each $i \in \mathbb{N}$ and the union of such subsets over all $i$ is $\mathcal{M}(H, J, \gamma)$.

Let $\mathcal{J}_{H}^{\text {reg }} \subset \mathcal{J}^{\Sigma}\left(J^{\#}, \check{C}\right)$ be the subspace of $H$-regular almost complex structures as in Definition 9.7. This is a ubiquitous subset by Proposition 9.13. Let $\left(N_{i}\right)_{i \in \mathbb{N}}$ be open subsets of $M$ satisfying $\cap_{i \in \mathbb{N}} N_{i}=V \cup([1+\epsilon / 8,1+\epsilon / 2] \times C)$. For each $i \in \mathbb{N}, J \in \mathcal{J}^{\Sigma}\left(J^{\#}, \check{C}\right)$ and $\gamma \in \Gamma(H)$ let $\mathcal{M}(i, J, \gamma) \subset K(i, J, \gamma)$ be the subset consisting of maps $u: \Sigma \longrightarrow M$ satisfying $u\left(U_{i}\right) \cap N_{i}=\emptyset$. Let

$$
\text { ev }: \Sigma \times \mathcal{M}(H, J, \gamma) \longrightarrow \Sigma \times M, \quad \operatorname{ev}(\sigma, u):=(\sigma, u(\sigma))
$$

be the natural evaluation map. For each $i \in \mathbb{N}, J \in \mathcal{J}_{H}^{\text {reg }}$ and $\gamma \in \Gamma(H)$, let $\mathcal{M}^{\operatorname{tr}}(i, J, \gamma) \subset$ $\mathcal{M}(H, J, \gamma)$ be the open subset consisting of maps $u$ for which there exists a neighborhood $N_{u}^{\prime}$ 
of $u$ in $\mathcal{M}(H, J, \gamma)$ so that $\left.\mathrm{ev}\right|_{S_{i} \times N_{u}^{\prime}}$ is transverse to $\left.f_{J}^{k}\right|_{E_{i, k, J}}$ for each $k \leq i$. Let $\mathcal{J}_{i, \gamma}^{\text {reg }} \subset \mathcal{J}_{H}^{\text {reg }}$ be the subset of almost complex structures $J$ satisfying $\mathcal{M}(i, J, \gamma) \subset \mathcal{M}^{\operatorname{tr}}(i, J, \gamma)$ for each $i \in \mathbb{N}$ and $\gamma \in \Gamma(H)$. Since $\mathcal{M}(i, J, \gamma)$ is compact for each $i \in \mathbb{N}, J \in \mathcal{J}_{i, \gamma}^{\text {reg }}$ and $\gamma \in \Gamma(H)$ and since transversality is an open condition so long as the corresponding domains are compact, we have that $J_{i, \gamma}^{\mathrm{reg}} \subset J_{H}^{\mathrm{reg}}$ is open. It is also dense by Lemma 9.24. Hence $\mathcal{J}_{i, \gamma}^{\mathrm{reg}}$ is a ubiquitous subset of $J^{\Sigma}\left(J^{\#}, \check{C}\right)$ for each $i \in \mathbb{N}, \gamma \in \Gamma(H)$ since modifying $J$ inside $U_{i} \times M$ does not change $\left.f_{J}^{k}\right|_{E_{i, k, J}}$ for all $k \leq i$. Hence $\mathcal{J}^{\text {reg }}:=\cap_{i, \gamma} \partial_{i, \gamma}^{\text {reg }}$ is ubiquitous in $J^{\Sigma}\left(J^{\#}, \check{C}\right)$.

Now let $J \in \mathcal{J}^{\text {reg }}$ and let $u \in \mathcal{M}(H, J, \gamma)$ for some $\gamma \in \Gamma(H)$. Since $\gamma \in \Gamma(H)$ and since $U_{i}$ eventually becomes disjoint from any compact subset of $\Sigma$ for $i$ large enough, there exists $i_{u} \in \mathbb{N}$ so that $u \in \mathcal{M}(i, J, \gamma)$ for each $i \geq i_{u}$. Since $J \in \mathcal{J}_{i, \gamma}^{\text {reg }}$ for each $i \geq i_{u}$, there is a neighborhood $N_{u, i}^{\prime}$ of $u$ in $\mathcal{M}\left(H, J^{\#}, \gamma\right)$ so that the evaluation map ev $\left.\right|_{\Sigma \times N_{u, i}^{\prime}}$ is transverse to $\left.f_{J}^{k}\right|_{E_{i, k, J}}$ for each $k \leq i$ and each $i \geq i_{u}$. Therefore since $S_{j} \subset S_{j+1}$ for all $j \in \mathbb{N}$, we get that ev is transverse to $\left.f_{J}^{k}\right|_{E_{i, k, J}}$ for each $i, k \in \mathbb{N}$ satisfying $k \leq i$ at each point $u \in \mathcal{M}(H, J, \gamma)$. Hence $\mathcal{J}^{\mathrm{reg}} \subset \mathcal{J}^{\mathrm{reg}}\left(H, J^{\#}, \check{C}\right)$ and so $\mathcal{J}^{\mathrm{reg}}\left(H, J^{\#}, \check{C}\right)$ is ubiquitous.

Also $M\left(H, J, \gamma^{\#}\right)$ is a manifold of dimension $k$ where $k$ is defined in (2.9) for each $\gamma^{\#} \in$ $\Gamma(H)$ and each $J \in \mathcal{J}^{\Sigma, \operatorname{reg}}\left(H, J^{\#}, \check{C}\right)$ by Proposition 9.18.

\section{Appendix C: Floer trajectories, Filtrations and Compactness.}

Throughout this section we will use the following notation (see Definitions 2.13 and 2.14):

- $\Sigma$ is a Riemann surface with $n_{-}$negative cylindrical ends and $n_{+}$positive cylindrical ends labeled by finite sets $I_{-}, I_{+}$respectively,

- $\iota_{j}: \mathbb{I}_{ \pm} \times \mathbb{T} \hookrightarrow \Sigma$ is the cylindrical end corresponding to $j$ for each $j \in I_{ \pm}$,

- $\beta$ is a $\Sigma$-compatible 1 -form and $\left(\kappa_{j}\right)_{j \in I_{-} \sqcup I_{+}}$are the weights of $\beta$ at each cylindrical end,

- $\check{C}$ is a contact cylinder with associated Liouville domain $D$,

- $H^{\#}:=\left(H^{j}\right)_{j \in I_{-} \sqcup I_{+}}$is a tuple of Hamiltonians and

- $J^{\#}:=\left(J^{j}\right)_{j \in I_{-} \sqcup I_{+}}$is a tuple of families of almost complex structures in $\mathcal{J}^{\mathbb{T}}\left(J_{0}, V, \omega\right)$.

Definition 10.1. Let $\left(\gamma_{j}\right)_{j \in I_{-} \sqcup I_{+}}=\left(\widetilde{\gamma}_{j}, \check{\gamma}_{j}\right)_{j \in I_{-} \sqcup I_{+}}$be capped loops where $\widetilde{\gamma}_{j}: \Sigma_{j} \longrightarrow M$ for each $j \in I_{-} \sqcup I_{+}$(Definition 2.1). A smooth map $u: \Sigma \longrightarrow M$ partially converges to $\left(\gamma_{j}\right)_{j \in I_{-} \sqcup I_{+}}$if there is a sequence $a_{1}^{j}, a_{2}^{j}, a_{3}^{j}, \cdots \in(0, \infty)$ tending to $a_{\infty}^{j} \in(0, \infty]$ for each $j \in I_{ \pm}$and a sequence of capped loops

$$
\left(\gamma_{j}^{k}\right)_{j \in I_{-} \sqcup I_{+}, k \in \mathbb{N}}=\left(\widetilde{\gamma}_{j}^{k}, \check{\gamma}_{j}^{k}\right)_{j \in I_{-} \sqcup I_{+}, k \in \mathbb{N}}
$$

where $\widetilde{\gamma}_{j}^{k}: \Sigma_{j}^{k} \longrightarrow M$ for each $j \in I_{-} \sqcup I_{+}, k \in \mathbb{N}$ so that

- $\widetilde{\gamma}_{j}^{k}\left(\check{\gamma}_{j}^{k}(t)\right)=u\left( \pm a_{k}^{j}, t\right)$ for each $t \in \mathbb{T}, j \in I_{ \pm}$, and $k \in \mathbb{N}$,

- the surface $u^{k}: S_{k} \longrightarrow M$ obtained by gluing

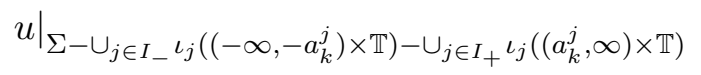

to each oriented surface $\widetilde{\gamma}_{j}^{k}, j \in I_{-} \sqcup I_{+}$is null-homologous for each $k \in \mathbb{N}$ and - $\gamma_{j}^{k} C^{0}$ converges to $\gamma_{j}$ in the space of capped loops as $k \longrightarrow \infty$ for each $j \in I_{-} \sqcup I_{+}$.

Note that if $u$ converges to capped 1-periodic orbits $\left(\gamma_{j}\right)_{j \in I_{-} \sqcup I_{+}}$as in Definition 2.14 then it partially converges to these capped 1-periodic orbits. Also note that $a_{\infty}^{j}$ does not have to be equal to $\infty$. 
Definition 10.2. Let $V$ be a vector space over $\mathbb{R}$ and $\omega_{V} \in \bigwedge^{2} V^{*}$. A linear complex structure $J_{V}: V \longrightarrow V$ is $\omega_{V}$-semi tame if $\omega_{V}\left(v, J_{V}(v)\right) \geq 0$ for all $v \in V$. An almost complex structure $J$ on $M$ is $\widetilde{\omega}$-semi tame for some $\widetilde{\omega} \in \Omega^{2}(M)$ if $\left.J\right|_{x}$ is $\left.\widetilde{\omega}\right|_{x}$-semi tame for all $x \in X$. Similarly, a smooth family of almost complex structures $\left(J_{\sigma}\right)_{\sigma \in \Sigma^{\prime}}$ is $\widetilde{\omega}$-semi tame if $J_{\sigma}$ is $\widetilde{\omega}$-semi tame for each $\sigma \in \Sigma^{\prime}$.

Lemma 10.3. Let $\widetilde{\omega} \in \Omega^{2}(M)$ be closed 2-form. Let

(1) $H:=\left(H_{\sigma}\right)_{\sigma \in \Sigma}$ be a $\Sigma$-compatible family of smooth functions (as in Definition 2.14) with limits $H^{\#}$,

(2) let $F:=\left(F_{\sigma}\right)_{\sigma \in \Sigma}$ be a smooth family of functions which is $\Sigma$-compatible with limits $F^{\#}=\left(F^{j}\right)_{j \in I_{-} \sqcup I_{+}}$and

(3) let $J:=\left(J_{\sigma}\right)_{\sigma \in \Sigma} \in \mathcal{J}^{\Sigma}\left(V, J_{0}, \omega\right)$ be a $\Sigma$-compatible family of almost complex structures with limits $J^{\#}$.

Suppose

- $H$ is $\widetilde{\omega}$-compatible where $F$ is the primitive associated to $(H, \widetilde{\omega})$ as in Definition 2.2, $J$ is $\widetilde{\omega}$-semi tame and

- $d\left(f^{x} \beta\right) \leq 0$ for all $x \in M$ where

$$
f^{x}: \Sigma \longrightarrow \mathbb{R}, \quad f^{x}(\sigma):=F_{\sigma}(x), \forall \sigma \in \Sigma .
$$

Then for any solution $u: \Sigma \longrightarrow M$ of the $(H, J)$-Floer equation which partially converges capped loops $\left(\gamma_{j}\right)_{j \in I_{-} \sqcup I_{+}}$, we have

$$
\sum_{j \in I_{-}} \mathcal{A}_{\kappa_{j} H^{j}, \widetilde{\omega}, \kappa_{j} F^{j}}\left(\gamma_{j}\right) \geq \sum_{j \in I_{+}} \mathcal{A}_{\kappa_{j} H^{j}, \widetilde{\omega}, \kappa_{j} F^{j}}\left(\gamma_{j}\right)
$$

(see Equation (2.4)).

Proof. The above inequality will follow from Stokes' formula. Let $\widehat{\omega}_{\beta}$ be a 2 -form on $\Sigma$ defined by

$$
\widehat{\omega}_{\beta}\left(Z_{1}, Z_{2}\right):=\widetilde{\omega}\left(d u\left(Z_{1}\right)-\beta\left(Z_{1}\right) X_{H_{\sigma}}, d u\left(Z_{2}\right)-\beta\left(Z_{2}\right) X_{H_{\sigma}}\right), \quad \forall Z_{1}, Z_{2} \in T_{\sigma} \Sigma, \sigma \in \Sigma .
$$

In order to prove our Lemma, we will show

(1) $\int_{\Sigma} \widehat{\omega}_{\beta} \geq 0$ and

(2) $\int_{\Sigma} \widehat{\omega}_{\beta} \leq \sum_{j \in I_{-}} \mathcal{A}_{\kappa_{j} H^{j}, \widetilde{\omega}, \kappa_{j} F^{j}}\left(\gamma_{j}\right)-\sum_{j \in I_{+}} \mathcal{A}_{\kappa_{j} H^{j}, \widetilde{\omega}, \kappa_{j} F^{j}}\left(\gamma_{j}\right)$.

We will now prove (1). If $\mathbf{j}$ is the complex structure on $\Sigma$, it is sufficient for us to show $\widehat{\omega}_{\beta}(Z, \mathbf{j} Z) \geq 0$ for all $Z \in T \Sigma$. Fix $z \in \Sigma$ and $Z \in T_{z} \Sigma$. Then $\widehat{\omega}_{\beta}(Z, \mathbf{j}(Z))=\widetilde{\omega}(d u(Z)-$ $\left.\beta(Z) X_{H_{\sigma}}, d u(\mathbf{j}(Z))-\beta\left(\mathbf{j}(Z) X_{H_{\sigma}}\right)\right)$. Since $u$ satisfies Floer's equation (2.8), we get that the above expression is equal to: $\widetilde{\omega}\left(d u(Z)-\beta(Z) X_{H_{\sigma}}, J_{\sigma}\left(d u(Z)-\beta(Z) X_{H_{\sigma}}\right)\right)$ which is $\geq 0$ since $J$ is $\widetilde{\omega}$-semi tame.

We now need to prove (2). We will do this by first modifying $\widehat{\omega}_{\beta}$ and then applying Stokes' formula. Let us look at a holomorphic coordinate chart $U$ inside $\Sigma$ with holomorphic coordinate $z=s+i t$ and consider the vectors $\partial_{s}:=\frac{\partial}{\partial s}, \partial_{t}:=\frac{\partial}{\partial t}$ at this point. Then

$$
\begin{gathered}
\widehat{\omega}_{\beta}\left(\partial_{s}, \partial_{t}\right)=\widetilde{\omega}\left(d u\left(\partial_{s}\right)-\beta\left(\partial_{s}\right) X_{H_{z}}, d u\left(\partial_{t}\right)-\beta\left(\partial_{t}\right) X_{H_{z}}\right)= \\
\widetilde{\omega}\left(d u\left(\partial_{s}\right), d u\left(\partial_{t}\right)\right)+\beta\left(\partial_{s}\right) \widetilde{\omega}\left(-X_{H_{z}}, d u\left(\partial_{t}\right)\right)- \\
\beta\left(\partial_{t}\right) \widetilde{\omega}\left(d u\left(\partial_{s}\right), X_{H_{z}}\right)+\beta\left(\partial_{s}\right) \beta\left(\partial_{t}\right) \widetilde{\omega}\left(X_{H_{z}}, X_{H_{z}}\right) \stackrel{(2.3)}{=} \\
\widetilde{\omega}\left(d u\left(\partial_{s}\right), d u\left(\partial_{t}\right)\right)+\beta\left(\partial_{s}\right) d F_{z}\left(d u\left(\partial_{t}\right)\right)-\beta\left(\partial_{t}\right) d F_{z}\left(d u\left(\partial_{s}\right)\right)= \\
\left(u^{*} \widetilde{\omega}+\beta \wedge u^{*} d F_{z}\right)\left(\partial_{s}, \partial_{t}\right) .
\end{gathered}
$$


Therefore

$$
\left.\widehat{\omega}_{\beta}\right|_{\sigma}=u^{*} \widetilde{\omega}+\beta \wedge u^{*} d F_{\sigma}, \quad \forall \sigma \in \Sigma .
$$

Define $\widehat{F}: \Sigma \longrightarrow \mathbb{R}, \widehat{F}(\sigma):=F_{\sigma}(u(\sigma))$. Since $d\left(f^{x} \beta\right) \leq 0$ for all $x \in M$, we get by Equation (10.2),

$$
\left.\widehat{\omega}_{\beta}\right|_{\sigma} \leq u^{*} \widetilde{\omega}-d(\widehat{F} \beta) .
$$

Let $\left(\bar{\gamma}_{j}\right)_{j \in I_{-} \sqcup I_{+}}$be the associated loops of the capped loops $\left(\gamma_{j}\right)_{j \in I_{-} \sqcup I_{+}}$. Since the maps $\gamma_{j}^{k}$ from Definition $10.1 C^{0}$ converge to $\gamma_{j}$ as $k$ tends to infinity, we have by Stokes' formula,

$$
\int_{\Sigma} d(\widehat{F} \beta)=\sum_{j \in I_{+}} \int_{0}^{1} \kappa_{j} F_{t}^{j}\left(\bar{\gamma}_{j}(t)\right) d t-\sum_{j \in I_{-}} \int_{0}^{1} \kappa_{j} F_{t}^{j}\left(\bar{\gamma}_{j}(t)\right) d t .
$$

Let $\gamma_{j}=\left(\widetilde{\gamma}_{j}, \check{\gamma}_{j}\right)$ be our capped loop where $\widetilde{\gamma}_{j}: \Sigma_{j} \longrightarrow M$ for each $j \in I_{-} \sqcup I_{+}$. Since the surface $u^{k}$ from Definition 10.1 is null-homologous for each $k$, we get the following equation

$$
\sum_{j \in I_{-}} \int_{\Sigma_{j}}\left(\widetilde{\gamma}_{j}\right)^{*} \widetilde{\omega}+\int_{\Sigma} u^{*} \widetilde{\omega}=\sum_{j \in I_{+}} \int_{\Sigma_{j}}\left(\widetilde{\gamma}_{j}\right)^{*} \widetilde{\omega}
$$

Therefore by Equations (10.3), (10.4), (10.5) and (2.4) we have

$$
\int_{\Sigma} \widehat{\omega}_{\beta} \leq \sum_{j \in I_{-}} \mathcal{A}_{\kappa_{j} H^{j}, \widetilde{\omega}, \kappa_{j} F^{j}}\left(\gamma_{j}\right)-\sum_{j \in I_{+}} \mathcal{A}_{\kappa_{j} H^{j}, \widetilde{\omega}, \kappa_{j} F^{j}}\left(\gamma_{j}\right) .
$$

Therefore (1) and (2) hold and we are done.

Definition 10.4. (see [Sch95, Definition 4.3.20]). Let $\gamma:=\left(\gamma_{j}\right)_{j \in I_{-} \sqcup I_{+}}$be non-degenerate capped 1-periodic orbits of $\left(\kappa_{j} H_{j}\right)_{j \in I_{-} \sqcup I_{+}}$. Let $H \in \mathcal{H}^{\Sigma}\left(H^{\#}, \check{C}\right)$ and $J \in \mathcal{J}^{\Sigma}\left(J^{\#}, \check{C}\right)$ as in Definition 2.14. A sequence $\left(u_{k}\right)_{k \in \mathbb{N}}$ in $\mathcal{M}(H, J, \gamma)$ (Definition 2.14) geometrically converges to a broken solution $(u, v)$ of degree 1 where $u: \Sigma \longrightarrow M, v: \mathbb{R} \times \mathbb{T} \longrightarrow M$ if there exists

- $m \in I_{ \pm}$,

- a non-degenerate capped 1-periodic orbit $\widehat{\gamma}$ of $\kappa_{m} H^{m}$ and

- a sequence $\left(s_{k}\right)_{k \in \mathbb{N}}$ tending to $\infty$ if $m \in I_{+}$and $-\infty$ if $m \in I_{-}$

so that $u_{k}$ converges in $C_{\text {loc }}^{\infty}$ to $u$ and $u_{k} \circ \iota_{m} \circ \tau_{k}$ converges in $C_{\text {loc }}^{\infty}$ to $v$ where

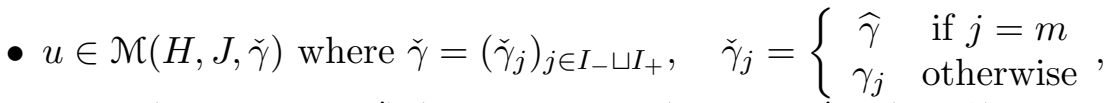

- $v \in \mathcal{M}\left(\kappa_{m} H_{m}, J^{m}, \gamma^{\prime}\right)$ (Definition 2.23) where $\gamma^{\prime}=\left(\gamma_{m}, \widehat{\gamma}\right)$ if $m \in I_{-}$and $\gamma^{\prime}=\left(\widehat{\gamma}, \gamma_{m}\right)$ if $m \in I_{+}$,

- $\tau_{k}$ is the map $\tau_{k}: \mathbb{I}_{s_{k}} \times \mathbb{T} \longrightarrow \mathbb{I}_{ \pm} \times \mathbb{T}, \quad \tau_{k}(s, t):=\left(s+s_{k}, t\right)$ for each $k \in \mathbb{N}$ where $\mathbb{I}_{s_{k}}:=\left[-s_{k}, \infty\right)$ if $m \in I_{+}$and $\mathbb{I}_{s_{k}}:=\left(-\infty,-s_{k}\right]$ if $m \in I_{-}$,

- $\left|\left(\check{\gamma}^{j}\right)_{j \in I_{-}}\right|-\left|\left(\check{\gamma}^{j}\right)_{j \in I_{+}}\right|=0$ and $|\widehat{\gamma}|-\left|\gamma_{m}\right|=1$ if $m \in I_{-}$and $\left|\gamma_{m}\right|-|\widehat{\gamma}|=1$ if $m \in I_{+}$.

We will call the capped 1-periodic orbit $\widehat{\gamma}$ the connecting orbit and $m \in I_{-} \sqcup I_{+}$the connecting index.

The following proposition is inspired by ideas from [CO18, Section 10.1].

Proposition 10.5. Suppose $I_{-}=\{\star\}$ is a single element set and (see Definitions 2.58 and 2.23)

- $\left(a_{-}^{j}, a_{+}^{j}\right) \in \operatorname{Sc}\left(Q_{-}^{j}\right) \times \operatorname{Sc}\left(Q_{+}^{j}\right)$ is a $\check{C}$-action interval,

- $\kappa_{j} H^{j} \in \mathcal{H}^{\mathbb{T}, \text { reg }}\left(\check{C}, a_{-}^{j}, a_{+}^{j}\right), J^{j} \in \mathcal{J}^{\mathbb{T}, \text { reg }}\left(\kappa_{j} H^{j}, \check{C}\right)$ and

- $\gamma_{j} \in \Gamma_{\check{C}, a_{-}^{j}, a_{+}^{j}}^{\mathbb{Z}}\left(H^{j}\right)$ 
for each $j \in I_{-} \sqcup I_{+}$. Suppose $Q_{ \pm}^{\star} \subset Q_{ \pm}^{j}$ for each $j \in I_{+}$and

$$
a_{-}^{\star} \leq\left.\sum_{j \in I_{+}} a_{-}^{j}\right|_{Q_{-}^{\star}}, \quad a_{+}^{\star} \leq\left. a_{+}^{j}\right|_{Q_{+}^{\star}}+\left.\sum_{j^{\prime} \in I_{+}-j} a_{-}^{j^{\prime}}\right|_{Q_{+}^{\star}}, \quad \forall j \in I_{+} .
$$

Suppose $\left|\gamma_{\star}\right|-\left|\left(\gamma_{j}\right)_{j \in I_{+}}\right|=1$. Let $H \in \mathcal{H}^{\Sigma}\left(H^{\#}, \check{C}\right)$ (Definition 2.14), $J \in J^{\Sigma, \operatorname{reg}}\left(H, J^{\#}, \check{C}\right)$ (Proposition 2.19) and let $\left(u_{k}\right)_{k \in \mathbb{N}}$ be a sequence in $\mathcal{M}(H, J, \gamma)$ where $\gamma=\left(\gamma_{j}\right)_{j \in I_{-} \sqcup I_{+}}$.

Then there is a subsequence $\left(u_{k_{j}}\right)_{j \in \mathbb{N}}$ which geometrically converges to a broken solution $(u, v)$ of degree 1 as in Definition 10.4 so that the connecting orbit $\widehat{\gamma}$ is an element of $\Gamma_{\tilde{C}, a_{-}^{m}, a_{+}^{m}}^{\mathbb{Z}}\left(H^{m}\right)$ where $m$ is the connecting index.

Proof. Suppose that there is some $m \in I_{ \pm}$and some sequence $s_{k} \in \mathbb{I}_{ \pm}$so that the sequence of maps

$$
l_{k}: \mathbb{T} \longrightarrow M, \quad t \rightarrow \iota_{m}\left(s_{k}, t\right)
$$

$C^{0}$ converges to a 1-periodic orbit $l: \mathbb{T} \longrightarrow M$. Let $\eta^{k}=\left(\widetilde{\eta}^{k}, \check{\eta}^{k}\right)$ be the unique capped loop with the property that its associated 1-periodic orbit is $l_{k}$ and where $\widetilde{\eta}^{k}$ is given by

- the catenation of $\left.\iota_{m}\right|_{\left(-\infty, s_{m}\right] \times \mathbb{T}}$ and $\widetilde{\gamma}^{m}$ if $m \in I_{-}$

- or the catenation of $\left.\iota_{j}\right|_{\left[s_{j}, \infty\right) \times \mathbb{T}}$ (with the opposite orientation) and $\widetilde{\gamma}^{m}$ if $m \in I_{+}$ where $\gamma_{m}=\left(\widetilde{\gamma}^{m}, \check{\gamma}^{m}\right)$.

By a repeated Gromov compactness argument (e.g. [Sch95, Theorem 4.3.22]) applied to cylinders and half cylinders, $\eta^{k}$ converges to a capped 1-periodic orbit $\widehat{\gamma}$ whose associated 1 -periodic orbit is $l$ after passing to a subsequence. Now by Lemma 10.3,

$$
\sum_{j \in I_{+}} \mathcal{A}_{H, \breve{C}}\left(\gamma_{j}\right) \leq \mathcal{A}_{H, \breve{C}}\left(\eta^{k}\right) \leq \mathcal{A}_{H, \check{C}}\left(\gamma_{\star}\right)
$$

if $m \in I_{-}$and

$$
\mathcal{A}_{H, \check{C}}\left(\gamma_{m}\right) \leq \mathcal{A}_{H, \check{C}}\left(\eta^{k}\right) \leq \mathcal{A}_{H, \check{C}}\left(\gamma_{\star}\right)-\sum_{j \in I_{+}-m} \mathcal{A}_{H, \check{C}}\left(\gamma_{j}\right)
$$

if $m \in I_{+}$for each $k \in \mathbb{N}$. Hence by Equation (10.6), $a_{-}^{m} \leq\left.\mathcal{A}_{H, \check{C}}\left(\eta^{k}\right)\right|_{Q_{-}^{m}}$ and $a_{+}^{m} \not \leq$ $\left.\mathcal{A}_{H, \check{C}}\left(\eta^{k}\right)\right|_{Q_{+}^{m}}$ for each $k \in \mathbb{N}$ and hence

$$
a_{-}^{m} \leq\left.\mathcal{A}_{H, \check{C}}(\widehat{\gamma})\right|_{Q_{-}^{m}}, \quad a_{+}^{m} \not \leq\left.\mathcal{A}_{H, \check{C}}(\widehat{\gamma})\right|_{Q_{+}^{m}} .
$$

Therefore $\widehat{\gamma}$ has to be non-degenerate. Hence by using the argument above one can show by [Sch95, Theorem 4.3.21] that (after passing to a subsequence) $\left(u_{k}\right)_{k \in \mathbb{N}}$ geometrically converges to a broken solution $(u, v)$ of degree 1 with connecting orbit $\widehat{\gamma}$ satisfying Equation (10.7).

Remark 10.6. There is also a parameterized version of Proposition 10.5 where we now have $H=\left(H_{s, \sigma}\right)_{s \in[0,1], \sigma \in \Sigma_{s}} \in \mathcal{H}^{\Sigma_{\bullet}}\left(H^{\#}, \check{C}\right)$ (Definition 2.20) and $J \in J^{\Sigma_{\bullet}, \operatorname{reg}}\left(H,\left(Y_{0}, Y_{1}\right), J^{\#}, \check{C}\right)$ (Proposition 2.21) for some smooth family of Riemann surfaces $\Sigma_{\bullet}:=\left(\Sigma_{t}\right)_{t \in[0,1]}$ where $Y_{j}=$ $\left(H_{j, s}\right)_{s \in \Sigma_{j}}$ for $j=0,1$ and $\left|\gamma_{\star}\right|-\left|\left(\gamma_{j}\right)_{j \in I_{+}}\right|=0$. The proof is also identical.

\section{Appendix D: Flatness of Novikov Rings.}

Throughout this section we will fix a finitely generated abelian group $(A, \cdot)$.

Definition 11.1. A rational polyhedral cone in $\left(A \otimes_{\mathbb{Z}} \mathbb{R}\right)^{*}$ is a cone of the form

$$
Q:=\left\{\sum_{i=0}^{k} r_{i} w_{i}: r_{0}, \cdots, r_{k} \geq 0\right\} \subset\left(A \otimes_{\mathbb{Z}} \mathbb{R}\right)^{*}
$$

for some fixed elements $w_{0}, \cdots, w_{k} \in\left(A \otimes_{\mathbb{Z}} \mathbb{Q}\right)^{*} \subset\left(A \otimes_{\mathbb{Z}} \mathbb{R}\right)^{*}$ called generators of $Q$. 
Such a cone is closed and hence if, in addition, this cone is salient (Definition 2.52) then we can define the Novikov rings $\Lambda_{\mathbb{K}}^{A, Q}$ and $\Lambda_{\mathbb{K}}^{A, Q,+}$ as in Definition 2.54. The aim of this section is to prove:

Proposition 11.2. Suppose $\mathbb{K}$ is Noetherian and let $Q_{0}, Q_{1} \subset\left(A \otimes_{\mathbb{Z}} \mathbb{R}\right)^{*}$ be salient rational polyhedral cones satisfying $Q_{1} \subset Q_{0}$. Then $\Lambda_{\mathbb{K}}^{A, Q_{1}}$ is a flat $\Lambda_{\mathbb{K}}^{A, Q_{0}}$-module.

The key idea of the proof is to show that there are appropriate subalgebras of $\Lambda^{A, Q_{j}}$, $j=0,1$ so that one is the completion of the other (Lemma 11.15) and so that appropriate localizations of them recover $\Lambda^{A, Q_{j}}, j=0,1$ (Lemma 11.16). Before we prove Proposition 11.2, we need a few definitions and lemmas. Throughout this section we will assume that our ring $\mathbb{K}$ is Noetherian.

Definition 11.3. Let $Q \subset\left(A \otimes_{\mathbb{Z}} \mathbb{R}\right)^{*}$ be a closed salient cone. For each $x \in A$, let $F_{x}^{Q}$ be the free $\mathbb{K}$-module generated by elements of the set $S_{x}^{Q}:=\left\{a \in A: x \preceq_{Q} a\right\}$ (Definition 2.54).

Remark 11.4. When $x=0, F_{0}^{Q}$ is a $\mathbb{K}$-algebra with multiplication induced from the product - on $A$ and $F_{x}^{Q}$ is an ideal in $F_{0}^{Q}$ for each $x \in A$ satisfying $0 \preceq_{Q} x$.

Definition 11.5. If $R$ is a ring and $x \in R$, then we write $(x)_{R}$ for the ideal generated by $x$. If it is clear which ring $x$ lives in, then we write $(x)=(x)_{R}$.

Lemma 11.6. Let $Q \subset\left(A \otimes_{\mathbb{Z}} \mathbb{R}\right)^{*}$ be a closed salient cone. Then we have an equality of ideals $\left(F_{x}^{Q}\right)^{m}=\left(x^{m}\right)$ in the $\mathbb{K}$-algebra $F_{0}^{Q}$ for each $x \in A, m>0$ satisfying $0 \preceq_{Q} x$.

Proof. Let $x \in A$ satisfy $0 \preceq_{Q} x$ and let $m>0$. Since $x \in F_{x}^{Q}$, we have $\left(x^{m}\right) \subset\left(F_{x}^{Q}\right)^{m}$. Now let $a \in A$ satisfy $x \preceq_{Q} a$. Then $0 \preceq_{Q} a \cdot x^{-1}$ which means that $a \cdot x^{-1} \in F_{0}^{Q}$. Hence $a^{m}=\left(a \cdot x^{-1}\right)^{m} x^{m} \in\left(x^{m}\right)$. Since $\left(F_{x}^{Q}\right)$ is an ideal generated by elements $a^{m}$ satisfying $x \preceq_{Q} a$, we then get that $\left(x^{m}\right)=\left(F_{x}^{Q}\right)^{m}$.

Definition 11.7. Let $Q \subset\left(A \otimes_{\mathbb{Z}} \mathbb{R}\right)^{*}$ be a closed salient cone. A $Q$-cofinal element is an element $y \in A$ satisfying $0 \preceq_{Q} y$ so that the sequence $\left(y^{n}\right)_{n \in \mathbb{N}}$ is cofinal in $\left(A, \preceq_{Q}\right)$.

Lemma 11.8. Let $Q_{0}, Q_{1} \subset\left(A \otimes_{\mathbb{Z}} \mathbb{R}\right)^{*}$ be closed salient cones so that $Q_{1} \subset Q_{0}$. Then there exists $y \in A$ which is both a $Q_{0}$ and $Q_{1}$-cofinal element.

Proof. Since $Q_{0}$ is closed and salient and $A$ is finitely generated, there exist $y_{0}, \cdots, y_{k} \in A$ generating $A$ as a group and satisfying $0 \preceq Q_{0} y_{i}$ for each $i=0, \cdots, k$. Then $0 \preceq Q_{1} y_{i}$ for each $i=0, \cdots, k$ as well since $Q_{1} \subset Q_{0}$. Define $y:=\prod_{i=0}^{k} y_{i}$. Now suppose $x \in A$ satisfies $0 \preceq Q_{j} x$ for some $j=0,1$. Then there exists $l_{0}, \cdots, l_{k} \in \mathbb{Z}$ so that $x=\prod_{i=0}^{k} y_{i}^{l_{i}}$. Then $0 \preceq Q_{j} x^{-1} \cdot y^{\max _{i=0}^{k}\left|l_{i}\right|}$ and hence $x \preceq Q_{j} y^{\max _{i=0}^{k}\left|l_{i}\right|}$. Therefore $y$ is a $Q_{j}$-cofinal element.

Definition 11.9. Let $R$ be a ring and $I \subset R$ an ideal. We define the completion of $R$ along $I$ to be the inverse limit of rings

$$
\widehat{R}_{I}:=\lim _{m \in \mathbb{N}} R / I^{m} .
$$

Lemma 11.10. Let $Q \subset\left(A \otimes_{\mathbb{Z}} \mathbb{R}\right)^{*}$ be a closed salient cone and let y be a $Q$-cofinal element. The natural inclusion map of $\mathbb{K}$-algebras $F_{0}^{Q} \hookrightarrow \Lambda_{\mathbb{K}}^{A, Q,+}$ extends to an isomorphism

$$
\widehat{F_{0}^{Q}} \stackrel{\cong}{\longrightarrow} \Lambda_{\mathbb{K}}^{A, Q,+} \text {. }
$$


Proof. By Lemma 11.6 combined with the fact that $y$ is a $Q$-cofinal element, we have

$$
\Lambda_{\mathbb{K}}^{A, Q,+}=\lim _{m \in \mathbb{N}} F_{0}^{Q} /\left(y^{m}\right) \text {. }
$$

by Equation (2.13). This proves our lemma since $\left(y^{m}\right)=(y)^{m}$ for each $m>0$.

Definition 11.11. A multiplicative subset in a commutative ring $R$ is a subset closed under multiplication. For a multiplicative subset $S \subset R$, we define the localization $S^{-1} R$ of $R$ along $S$ to be the set of equivalence classes of pairs $(r, s) \in R \times S$ so that $\left(r_{1}, s_{1}\right) \sim\left(r_{2}, s_{2}\right)$ if and only if there exists a $t \in S$ so that $t\left(r_{1} s_{2}-r_{2} s_{1}\right)=0$, where addition and multiplication are defined via the formulas

$$
\left(r_{1}, s_{1}\right)+\left(r_{2}, s_{2}\right)=\left(r_{1} s_{2}+r_{2} s_{1}, s_{1} s_{2}\right), \quad\left(r_{1}, s_{1}\right)\left(r_{2}, s_{2}\right)=\left(r_{1} r_{2}, s_{1} s_{2}\right) .
$$

If $x \in R$, we define $S_{x} \subset R$ to be the smallest multiplicative subset containing $x$.

Lemma 11.12. Let $Q \subset\left(A \otimes_{\mathbb{Z}} \mathbb{R}\right)^{*}$ be a closed salient cone and let y be a $Q$-cofinal element. Then the natural inclusion map $\Lambda_{\mathbb{K}}^{A, Q,+} \hookrightarrow \Lambda_{\mathbb{K}}^{A, Q}$ extends to an isomorphism of $\mathbb{K}$-algebras

$$
S_{y}^{-1} \Lambda_{\mathbb{K}}^{A, Q,+} \stackrel{\cong}{\longrightarrow} \Lambda_{\mathbb{K}}^{A, Q} \text {. }
$$

Proof. Since $F_{x_{0} x_{1}}^{Q}=x_{0} F_{x_{1}}^{Q}$ for each $x_{0}, x_{1} \in A$ and since $y^{-1}$ is $-Q$-cofinal, we have a natural isomorphism

$$
\Lambda_{\mathbb{K}}^{A, Q}=\lim _{m \in \mathbb{N}} y^{-m} \Lambda_{\mathbb{K}}^{A, Q,+}
$$

by Equations (2.12) and (2.13) where the morphisms in the corresponding directed system are the inclusion maps. Therefore since $y$ is invertible in $\Lambda_{\mathbb{K}}^{A, Q}$, we get by the definition of direct limit (Definition 2.33) that elements of the ring (11.3) are equivalence classes of pairs $\left(x, y^{m}\right), x \in \Lambda_{\mathbb{K}}^{A, Q,+}, m>0$ where $\left(x, y^{m}\right) \sim\left(x^{\prime}, y^{m^{\prime}}\right)$ iff $x y^{m^{\prime}+k}-x^{\prime} y^{m+k}=0$ for some $k \geq 0$ and where addition and multiplication satisfy formulas which are similar to (11.1). This proves our lemma.

We get the following immediate corollary of Lemmas 11.10 and 11.12.

Corollary 11.13. Let $Q_{0}, Q_{1} \subset\left(A \otimes_{\mathbb{Z}} \mathbb{R}\right)^{*}$ be closed salient cones so that $Q_{1} \subset Q_{0}$ and let $y \in A$ be a $Q_{j}$-cofinal element for $j=0,1$. Then we have the following commutative diagram of $\mathbb{K}$-algebras:

$$
\begin{aligned}
& S_{y}^{-1}\left(\widehat{F_{0}^{Q_{0}}}{ }_{(y)}\right) \stackrel{\cong}{\rightrightarrows} \Lambda_{\mathbb{K}}^{A, Q_{0}} \\
& S_{y}^{-1}\left(\stackrel{\downarrow}{F_{0}^{Q_{1}}(y)}\right) \stackrel{\downarrow}{\stackrel{\downarrow}{A}, Q_{1}}
\end{aligned}
$$

From now on, until the end of this section, we will let $Q_{0}, Q_{1} \subset\left(A \otimes_{\mathbb{Z}} \mathbb{R}\right)^{*}$ be closed salient cones so that $Q_{1} \subset Q_{0}$ and let $y$ be a $Q_{j}$-cofinal element for $j=0,1$ (see Lemma 11.8). We will also define $R^{j}:=F_{0}^{Q_{j}}$ and $I_{j}:=(y)_{R^{j}}$ (Definition 11.5) for $j=0,1$.

Lemma 11.14. The natural map

$$
\mathbb{K}[A]=S_{y}^{-1} R^{0} \longrightarrow S_{y}^{-1}{\widehat{R^{0}}}_{I_{0}}
$$

is injective.

Proof. The natural map $R^{0} \longrightarrow{\widehat{R^{0}}}_{I_{0}}$ is injective since $\cap_{m \in \mathbb{N}} I_{0}^{m}=0$. Hence (11.4) is injective by [Sta18, Tag 00CS]. 
Lemma 11.15. The map

$$
R^{1} \otimes_{R^{0}}{\widehat{R^{0}}}_{I_{0}} \longrightarrow{\widehat{R^{1}}}_{I_{1}}
$$

induced by the natural inclusion maps extends to an isomorphism

$$
\left(R^{1} \widehat{\otimes_{R^{0}} \widehat{R^{0}}} I_{0}\right)_{(1 \otimes y)} \stackrel{\cong}{\longrightarrow} \widehat{R^{1}} I_{1} .
$$

Proof. The map (11.5) induces an isomorphism

$$
\left(R^{1} \otimes_{R^{0}} \widehat{R}_{I_{0}}\right) /\left(1 \otimes y^{m}\right)=\left(R^{1} \otimes_{R^{0}} R^{0}\right) /\left(1 \otimes y^{m}\right) \cong R^{1} /\left(y^{m}\right)
$$

for each $m>0$. Taking the inverse limit as $m \rightarrow \infty$ of this isomorphism gives us the isomorphism (11.6).

Note that we have natural inclusion maps

$$
R^{1} \hookrightarrow S_{y}^{-1} R^{1}=\mathbb{K}[A]=S_{y}^{-1} R^{0} \hookrightarrow S_{y}^{-1} \widehat{R}^{0} I_{0}
$$

since $y$ is not a zero divisor in $R^{0}$ or $R^{1}$ and also by Lemma 11.14.

Lemma 11.16. The map

$$
\Psi: R^{1} \otimes_{R^{0}} \widehat{R^{0}} I_{0} \longrightarrow S_{y}^{-1} \widehat{R}^{0} I_{0}
$$

induced by the natural inclusion map (11.7) extends to an isomorphism

$$
\Phi: S_{1 \otimes y}^{-1}\left(R^{1} \otimes_{R^{0}}{\widehat{R^{0}}}_{I_{0}}\right) \stackrel{\cong}{\longrightarrow} S_{y}^{-1}\left({\widehat{R^{0}}}_{I_{0}}\right) .
$$

Proof. Since the map (11.8) sends $1 \otimes y$ to $y$, we get that the map $\Phi$ is well defined. Also the composition of the map $S_{1 \otimes y}^{-1}\left(R^{0} \otimes_{R^{0}} \widehat{R}_{I_{0}}\right) \longrightarrow S_{1 \otimes y}^{-1}\left(R^{1} \otimes_{R^{0}} \widehat{R}^{0} I_{0}\right)$ with $\Phi$ is an isomorphism and hence $\Phi$ is surjective. Finally, suppose $c \in \operatorname{ker}(\Phi)$. Then, there exists $m>0$ so that $c^{\prime}:=(1 \otimes y)^{m} c \in R^{1} \otimes_{R^{0}} \widehat{R^{0}} I_{0}$. Since $y$ is a $Q_{0^{-}}$-cofinal element, we have

$$
(y \otimes 1)^{m^{\prime}} c^{\prime} \in R^{0} \otimes_{R^{0}}{\widehat{R^{0}}}_{I_{0}}={\widehat{R^{0}}}_{I_{0}}
$$

for some large $m^{\prime}>0$. Since $y$ is not a zero divisor in $\widehat{R^{0}} I_{0}$, we get that the inclusion map $\widehat{R}^{0} I_{0} \hookrightarrow S_{y}^{-1} \widehat{R}_{I_{0}}$ is an injection and so the element (11.10) is zero. Hence

$$
(1 \otimes y)^{m+m^{\prime}} c=(y \otimes 1)^{m^{\prime}} c^{\prime}=0 \in R^{1} \otimes_{R^{0}}{\widehat{R^{0}}}_{I_{0}} .
$$

Since $1 \otimes y$ is invertible in $S_{1 \otimes y}^{-1}\left(R^{1} \otimes_{R^{0}}{\widehat{R^{0}}}_{I_{0}}\right)$, this implies that $c=0$ and hence $\Phi$ is injective. Hence $\Phi$ is an isomorphism.

Proof of Proposition 11.2. Since $Q_{j}$ is a rational polyhedral cone we get that $R^{j}$ is a finitely generated $\mathbb{K}$-algebra by Gordan's lemma [Ful93, Proposition 1, Section 1.2] and hence is Noetherian by [Sta18, Tag 00FN] for $j=0,1$ since $\mathbb{K}$ is Noetherian. Hence $R^{1} \otimes_{R^{0}} \widehat{R^{0}} I_{0}$ is Noetherian by [Sta18, Tag 0CY6], [Sta18, Tag 00FN] and [Sta18, Tag 05GH]. Therefore $\left(R^{1} \widehat{\otimes_{R^{0}} \widehat{R^{0}}} I_{0}\right)_{(1 \otimes y)}$ is a flat $R^{1} \otimes_{R^{0}} \widehat{R^{0}} I_{0}$-module by [Sta18, Tag 00MB] and [Sta18, Tag 00HT (1)]. Hence $\widehat{R}^{1} I_{1}$ is a flat $R^{1} \otimes_{R^{0}}{\widehat{R^{0}}}_{I_{0}}$-module by Lemma 11.15. Therefore since $S_{y}^{-1} \widehat{R}_{I_{1}}$ is a flat $R^{1} \otimes_{R^{0}} \widehat{R^{0}} I_{0}$-module by [Sta18, Tag 00HT (1)] combined with [Sta18, Tag 00HC] we get that $S_{y}^{-1} \widehat{R}_{I_{1}}$ is a flat $S_{1 \otimes y}^{-1}\left(R^{1} \otimes_{R^{0}}{\widehat{R^{0}}}_{I_{0}}\right)$-module by [Sta18, Tag 00HT (2)]. Hence $S_{y}^{-1} \widehat{R}^{1} I_{1}$ is a flat $S_{y}^{-1}\left(\widehat{R}_{I_{0}}\right)$-module by Lemma 11.16. Our proposition now follows from Corollary 11.13 . 


\section{REFERENCES}

[AD14] Michèle Audin and Mihai Damian. Morse theory and Floer homology. Universitext. Springer, London; EDP Sciences, Les Ulis, 2014. ISBN 978-1-4471-5495-2; 978-1-4471-5496-9; 978-2-75980704-8. doi:10.1007/978-1-4471-5496-9. Translated from the 2010 French original by Reinie Erné. $\uparrow 28, \uparrow 31, \uparrow 32, \uparrow 33, \uparrow 48$

[Bat99] Victor Batyrev. Birational Calabi-Yau $n$-folds have equal Betti numbers. 264:1-11, 1999. doi: 10.1017/CBO9780511721540.002. $\uparrow 2, \uparrow 88$

[Bei87] Aleksandr Beilinson. How to glue perverse sheaves. In K-theory, arithmetic and geometry (Moscow, 1984-1986), volume 1289 of Lecture Notes in Math., pages 42-51. Springer, Berlin, 1987. doi: 10.1007/BFb0078366. $\uparrow 18$

[CE12] Kai Cieliebak and Yakov Eliashberg. From Stein to Weinstein and back, volume 59 of American Mathematical Society Colloquium Publications. American Mathematical Society, Providence, RI, 2012. ISBN 978-0-8218-8533-8. Symplectic geometry of affine complex manifolds. $\uparrow 70$

[CFH95] Kai Cieliebak, Andreas Floer, and Helut Hofer. Symplectic homology II. A general construction. Math. Z., 218(1):103-122, 1995. ISSN 0025-5874. doi:10.1007/BF02571891. ^3, 个6

[CFHW96] Kai Cieliebak, Andreas Floer, Helut Hofer, and Krzysztof Wysocki. Applications of symplectic homology II: Stability of the action spectrum. Math. Z., 223(1):27-45, 1996. ISSN 0025-5874. doi:10.1007/PL00004267. $\uparrow 51$

[CO18] Kai Cieliebak and Alexandru Oancea. Symplectic homology and the Eilenberg-Steenrod axioms. Algebr. Geom. Topol., 18(4):1953-2130, 2018. ISSN 1472-2747. doi:10.2140/agt.2018.18.1953. Appendix written jointly with Peter Albers. $\uparrow 3, \uparrow 6, \uparrow 104$

[CZ84] Charles Conley and Eduard Zehnder. Morse-type index theory for flows and periodic solutions for Hamiltonian equations. Comm. Pure Appl. Math., 37(2):207-253, 1984. ISSN 0010-3640. doi: 10.1002/cpa.3160370204. $\uparrow 15$

[DL01] Jan Denef and François Loeser. Geometry on arc spaces of algebraic varieties. In European Congress of Mathematics, Vol. I (Barcelona, 2000), volume 201 of Progr. Math., pages 327-348. Birkhäuser, Basel, 2001. $\uparrow 2$

[EM02] Yakov Eliashberg and Nikolai Mishachev. Introduction to the h-principle, volume 48 of Graduate Studies in Mathematics. American Mathematical Society, Providence, RI, 2002. ISBN 0-82183227-1. doi:10.1090/gsm/048. $\uparrow 81, \uparrow 85$

[FH94] Andreas Floer and Helmut Hofer. Symplectic homology I. Open sets in $\mathbf{C}^{n}$. Math. Z., 215(1):37-88, 1994. ISSN 0025-5874. doi:10.1007/BF02571699. $\uparrow 5$

[Flo88] Andreas Floer. The unregularized gradient flow of the symplectic action. Comm. Pure Appl. Math., 41(6):775-813, 1988. ISSN 0010-3640. doi:10.1002/cpa.3160410603. $\uparrow 13$

[Fri91] Robert Friedman. On threefolds with trivial canonical bundle. In Complex geometry and Lie theory (Sundance, UT, 1989), volume 53 of Proc. Sympos. Pure Math., pages 103-134. Amer. Math. Soc., Providence, RI, 1991. doi:10.1090/pspum/053/1141199. $\uparrow 2$

[FTMZ14] Mohammad Farajzadeh-Tehrani, Mark McLean, and Aleksey Zinger. Normal crossings divisors and configurations for symplectic topology. arxiv.org/abs/1410.0609, pages 1-66, 2014. $\uparrow 72, \uparrow 73$, $\uparrow 77$

[Ful93] William Fulton. Introduction to toric varieties, volume 131 of Annals of Mathematics Studies. Princeton University Press, Princeton, NJ, 1993. ISBN 0-691-00049-2. doi:10.1515/9781400882526. The William H. Roever Lectures in Geometry. $\uparrow 108$

[GH94] Phillip Griffiths and Joseph Harris. Principles of algebraic geometry. Wiley Classics Library. John Wiley \& Sons, Inc., New York, 1994. ISBN 0-471-05059-8. doi:10.1002/9781118032527. Reprint of the 1978 original. $\uparrow 75, \uparrow 76, \uparrow 88, \uparrow 89, \uparrow 90, \uparrow 91$

[Gin10] Viktor Ginzburg. The Conley conjecture. Ann. of Math. (2), 172(2):1127-1180, 2010. ISSN 0003486X. doi:10.4007/annals.2010.172.1129. $\uparrow 49$

[GPS15] Sheel Ganatra, Timothy Perutz, and Nick Sheridan. Mirror symmetry: from categories to curve counts. arXiv preprint arXiv:1510.03839, 2015. $\uparrow 3$

[Gro15] Yoel Groman. Floer theory on open manifolds. arXiv preprint arXiv:1510.04265, 2015. $\uparrow 3, \uparrow 6, \uparrow 36$

[GS96] Henri Gillet and Christophe Soulé. Descent, motives, and K-theory. Journal Fur Die Reine Und Angewandte Mathematik, 478:127-176, 1996. $\uparrow 2$

[Gür08] Başak Gürel. Totally non-coisotropic displacement and its applications to Hamiltonian dynamics. Communications in Contemporary Mathematics, 10(06):1103-1128, 2008. $\uparrow 81$ 
[Gut14] Jean Gutt. Generalized Conley-Zehnder index. Ann. Fac. Sci. Toulouse Math. (6), 23(4):907-932, 2014. ISSN 0240-2963. doi:10.5802/afst.1430. $\uparrow 15$

[Hir64] Heisuke Hironaka. Resolution of singularities of an algebraic variety over a field of characteristic zero. I, II. Ann. of Math. (2) 79 (1964), 109-203; ibid. (2), 79:205-326, 1964. ISSN 0003-486X. $\uparrow 67, \uparrow 68, \uparrow 80$

[HS95] Helmut Hofer and Dietmar Salamon. Floer homology and Novikov rings. In The Floer memorial volume, volume 133 of Progr. Math., pages 483-524. Birkhäuser, Basel, 1995. $\uparrow 94$

[ILLW12] Yoshihiro Iwao, Yuan-Pin Lee, Hui-Wen Lin, and Chin-Lung Wang. Invariance of Gromov-Witten theory under a simple flop. J. Reine Angew. Math., 663:67-90, 2012. ISSN 0075-4102. doi:10.1515/ CRELLE.2011.097. $\uparrow 3$

[Kat00] Kazuya Kato. Existence theorem for higher local fields. In Invitation to higher local fields (Münster, 1999), volume 3 of Geom. Topol. Monogr., pages 165-195. Geom. Topol. Publ., Coventry, 2000. doi:10.2140/gtm.2000.3.165. $\uparrow 18$

[Kaw02] Yujiro Kawamata. D-equivalence and K-equivalence. J. Differential Geom., 61(1):147-171, 2002. ISSN 0022-040X. $\uparrow 2$

[Kaw08] Yujiro Kawamata. Flops connect minimal models. Publ. Res. Inst. Math. Sci., 44(2):419-423, 2008. ISSN 0034-5318. doi:10.2977/prims/1210167332. $\uparrow 2$

[KM98] János Kollár and Shigefumi Mori. Birational geometry of algebraic varieties, volume 134 of Cambridge Tracts in Mathematics. Cambridge University Press, Cambridge, 1998. ISBN 0-521-63277-3. With the collaboration of C. H. Clemens and A. Corti, Translated from the 1998 Japanese original. $\uparrow 88$

[Kon95] Maxim Kontsevich. Grothendieck ring of motives and related rings. Lecture at Orsay, December 7, 1995. $\uparrow 2$

[LLQW16] Yuan-Pin Lee, Hui-Wen Lin, Feng Qu, and Chin-Lung Wang. Invariance of quantum rings under ordinary flops III: A quantum splitting principle. Camb. J. Math., 4(3):333-401, 2016. ISSN 21680930. doi:10.4310/CJM.2016.v4.n3.a2. $\uparrow 2, \uparrow 3$

[LLW16a] Yuan-Pin Lee, Hui-Wen Lin, and Chin-Lung Wang. Invariance of quantum rings under ordinary flops I: Quantum corrections and reduction to local models. Algebr. Geom., 3(5):578-614, 2016. ISSN 2214-2584. doi:10.14231/AG-2016-026. $\uparrow 2, \uparrow 3$

[LLW16b] Yuan-Pin Lee, Hui-Wen Lin, and Chin-Lung Wang. Invariance of quantum rings under ordinary flops II: A quantum Leray-Hirsch theorem. Algebr. Geom., 3(5):615-653, 2016. ISSN 2214-2584. doi:10.14231/AG-2016-027. $\uparrow 2, \uparrow 3$

[LR01] An-Min Li and Yongbin Ruan. Symplectic surgery and Gromov-Witten invariants of Calabi-Yau 3-folds. Invent. Math., 145(1):151-218, 2001. ISSN 0020-9910. doi:10.1007/s002220100146. $\uparrow 2$

[LS94] François Laudenbach and Jean-Claude Sikorav. Hamiltonian disjunction and limits of Lagrangian submanifolds. International Mathematics Research Notices, 1994(4):161-168, 1994. $\uparrow 81$

[Mar00] Sibe Mardešić. Strong shape and homology. Springer Monographs in Mathematics. Springer-Verlag, Berlin, 2000. ISBN 3-540-66198-0. doi:10.1007/978-3-662-13064-3. ^18, ^23

[McL12a] Mark McLean. The growth rate of symplectic homology and affine varieties. Geom. Funct. Anal., 22(2):369-442, 2012. doi:10.1007/s0003901201587. $\uparrow 77$

[McL12b] Mark McLean. Local Floer homology and infinitely many simple Reeb orbits. Algebr. Geom. Topol., 12(4):1901-1923, 2012. ISSN 1472-2747. doi:10.2140/agt.2012.12.1901. $\uparrow 32$

[McL16] Mark McLean. Reeb orbits and the minimal discrepancy of an isolated singularity. Invent. Math.,

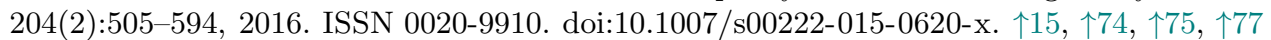

[Mor96] David R. Morrison. Beyond the Kähler cone. In Proceedings of the Hirzebruch 65 Conference on Algebraic Geometry (Ramat Gan, 1993), volume 9 of Israel Math. Conf. Proc., pages 361-376. Bar-Ilan Univ., Ramat Gan, 1996. $\uparrow 2, \uparrow 4$

[MS82] Sibe Mardešić and Jack Segal. Shape theory, volume 26 of North-Holland Mathematical Library. North-Holland Publishing Co., Amsterdam-New York, 1982. ISBN 0-444-86286-2. The inverse system approach. $\uparrow 18$

[MS98] Dusa McDuff and Dietmar Salamon. Introduction to symplectic topology. Oxford Mathematical Monographs. The Clarendon Press Oxford University Press, New York, second edition, 1998. ISBN 0-19-850451-9. $\uparrow 16, \uparrow 44, \uparrow 80, \uparrow 83, \uparrow 84, \uparrow 91, \uparrow 94$

[MS04] Dusa McDuff and Dietmar Salamon. J-holomorphic curves and symplectic topology, volume 52 of American Mathematical Society Colloquium Publications. American Mathematical Society, Providence, RI, 2004. ISBN 0-8218-3485-1. $\uparrow 95, \uparrow 100, \uparrow 101$ 
[Oan04] Alexandru Oancea. A survey of Floer homology for manifolds with contact type boundary or symplectic homology. In Symplectic geometry and Floer homology. A survey of the Floer homology for manifolds with contact type boundary or symplectic homology, volume 7 of Ensaios Mat., pages 51-91. Soc. Brasil. Mat., Rio de Janeiro, 2004. $\uparrow 49$

[OZ11] Yong-Guen Oh and Ke Zhu. Floer trajectories with immersed nodes and scale-dependent gluing. Journal of Symplectic Geometry, 9(4):483-636, 2011. $\uparrow 48$

[Pop93] Georgi Popov. Length spectrum invariants of Riemannian manifolds. Math. Z., 213(2):311-351, 1993. ISSN 0025-5874. doi:10.1007/BF03025724. $\uparrow 63, \uparrow 94$

[PSS96] Sergey Piunikhin, Dietmar Salamon, and Matthias Schwarz. Symplectic Floer-Donaldson theory and quantum cohomology. In Contact and symplectic geometry (Cambridge, 1994), volume 8 of Publ. Newton Inst., pages 171-200. Cambridge Univ. Press, Cambridge, 1996. $\uparrow 48$

[Rit13] Alexander Ritter. Topological quantum field theory structure on symplectic cohomology. J. Topol., 6(2):391-489, 2013. ISSN 1753-8416. doi:10.1112/jtopol/jts038. $\uparrow 28, \uparrow 30, \uparrow 35$

[RS93] Joel Robbin and Dietmar Salamon. The Maslov index for paths. Topology, 32(4):827-844, 1993. ISSN 0040-9383. doi:10.1016/0040-9383(93)90052-W. $\uparrow 15$

[Rua96] Yongbin Ruan. Topological sigma model and Donaldson-type invariants in Gromov theory. Duke Math. J., 83(2):461-500, 1996. ISSN 0012-7094. doi:10.1215/S0012-7094-96-08316-7. ^3

[Rua99] Yongbin Ruan. Surgery, quantum cohomology and birational geometry. In Northern California Symplectic Geometry Seminar, volume 196 of Amer. Math. Soc. Transl. Ser. 2, pages 183-198. Amer. Math. Soc., Providence, RI, 1999. $\uparrow 2, \uparrow 3$

[Sch95] Matthias Schwarz. Cohomology operations from S1-cobordisms in Floer homology. Phd thesis,

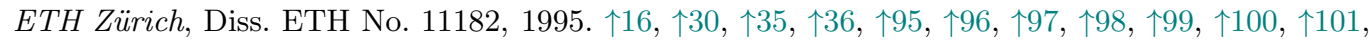
$\uparrow 104, \uparrow 105$

[Sei08] Paul Seidel. A biased view of symplectic cohomology. In Current developments in mathematics, 2006, pages 211-253. Int. Press, Somerville, MA, 2008. $\uparrow 68$

[Sei16] Paul Seidel. Connections on equivariant Hamiltonian Floer cohomology. arXiv preprint arXiv:1612.07460, 2016. $\uparrow 3$

[Sha13] Igor R. Shafarevich. Basic algebraic geometry 1. Springer, Heidelberg, third edition, 2013. ISBN 978-3-642-37955-0; 978-3-642-37956-7. Varieties in projective space. $\uparrow 88$

[Sta18] The Stacks Project Authors. Stacks Project. https://stacks.math.columbia.edu, 2018. $\uparrow 43$, $\uparrow 107, \uparrow 108$

[Ush11] Michael Usher. Deformed Hamiltonian Floer theory, capacity estimates and Calabi quasimorphisms. Geometry \& Topology, 15(3):1313-1417, 2011. $\uparrow 17$

[Var18] Umut Varolgunes. Mayer-Vietoris property for relative symplectic cohomology. arXiv preprint arXiv:1806.00684, 2018. $\uparrow 3, \uparrow 6$

[Ven18] Sara Venkatesh. Rabinowitz Floer homology and mirror symmetry. J. Topol., 11(1):144-179, 2018. ISSN 1753-8416. doi:10.1112/topo.12050. $\uparrow 3, \uparrow 6$

[Wan02] Chin-Lung Wang. K-equivalence in birational geometry. arXiv preprint math/0204160, $2002 . \uparrow 2$

[Wei94] Charles Weibel. An introduction to homological algebra, volume 38 of Cambridge Studies in Advanced Mathematics. Cambridge University Press, Cambridge, 1994. ISBN 0-521-43500-5; 0-52155987-1. doi:10.1017/CBO9781139644136. $\uparrow 4, \uparrow 23, \uparrow 48, \uparrow 66$

[Whi65] Hassler Whitney. Tangents to an analytic variety. Ann. of Math. (2), 81:496-549, 1965. ISSN 0003-486X. doi:10.2307/1970400. $\uparrow 81$ 